University of Tennessee Health Science Center UTHSC Digital Commons

\title{
Fear Learning and the Olfactory Bulb: Neural Correlates of Behavioral Fear Generalization
}

Jordan Marie Ross

University of Tennessee Health Science Center

Follow this and additional works at: https://dc.uthsc.edu/dissertations

Part of the Neurosciences Commons

\section{Recommended Citation}

Ross, Jordan Marie (0000-0003-4345-0135), "Fear Learning and the Olfactory Bulb: Neural Correlates of Behavioral Fear Generalization" (2019). Theses and Dissertations (ETD). Paper 499. http://dx.doi.org/ 10.21007/etd.cghs.2019.0484.

This Dissertation is brought to you for free and open access by the College of Graduate Health Sciences at UTHSC Digital Commons. It has been accepted for inclusion in Theses and Dissertations (ETD) by an authorized administrator of UTHSC Digital Commons. For more information, please contact jwelch30@uthsc.edu. 


\title{
Fear Learning and the Olfactory Bulb: Neural Correlates of Behavioral Fear Generalization
}

\author{
Abstract \\ The exploration of how learning alters neural coding to guide behaviors remains fundamental to \\ neuroscience. At the most basic level, the ability for organisms to flexibly adapt to changing environments \\ and situations is paramount to biological success and often manifests in behavioral responses controlled \\ by neural activity. For example, organisms must modify their behavior to defensive responses in the face \\ of biological threat. Neural circuitry is involved in coordinating an initial defensive behavioral response but \\ must undergo reorganization in order to reliably employ defensive responses in subsequent encounters \\ based on a cue that signals imminent danger. One such form of this learning is associative fear learning, \\ in which an organism learns to associate an initially neutral stimulus, which by itself has no biological \\ relevance, with an innately fear-inducing stimulus. After temporally pairing the two stimuli, organisms \\ learn that the initially neutral stimulus predicts the fear-inducing stimulus such that encountering the \\ former induces similar behavioral responses as the latter." "Interestingly, associative fear learning is often \\ not specific and organisms display fear responses to completely neutral stimuli that have never been \\ associated with the innately fear-inducing stimulus. This aberrant expression of fear is referred to as \\ generalization and is a hallmark of many psychological disorders such as anxiety and post-traumatic \\ stress disorder. The underlying cause of fear generalization remains unknown; however, there are two \\ predominant theories. One theory is fear generalization arises as a better-safe-than-sorry strategy, \\ wherein sensory information remains perceptually segregated between fear predictive and neutral stimuli \\ but the organism responds to both the same way despite the perceptual information. The second theory \\ argues that learning alters sensory processing to make the stimuli more difficult to perceptually \\ distinguish, which cascades into behaviorally treating them as the same. The olfactory bulb (OB) \\ represents an ideal model system for studying the extent to which fear learning alters sensory coding in \\ ways that support failure of perceptual discrimination due to its odotopic organization that creates unique \\ "maps" of odor representation for each experience odor. Therefore, we used both wide-field and 2-photon \\ (2P) calcium imaging of the OBs of awake mice in combination with classical olfactory fear conditioning \\ to investigate how learning changes sensory representation of the conditioned odor, the initially neutral \\ odor specifically paired with fear-inducing foot shock, as well as neutral odors never paired with shock. \\ This allowed direct testing of the extent to which fear learning reorganizes olfactory processing in a \\ manner that supports the failure to discriminate hypothesis of fear generalization. Both wide-field and $2 \mathrm{P}$ \\ imaging revealed enhanced odor-evoked responses following fear learning that likely signal increased \\ salience of incoming sensory information. Furthermore, the responses evoked by neutral odors became \\ more similar to those evoked by the conditioned odor at both the population level (wide-field) and in a \\ specific subset of $O B$ output cells (2P), indicating neutral odors were more difficult to distinguish from the \\ conditioned odor. Importantly, the enhanced odor-evoked responses were not attributable to behavioral \\ state change nor top-down influence from the amygdala, the area widely believed to be involved in \\ aberrant fear. Together, this evidence supported the failure of perceptual discrimination at early stages of \\ sensory process as the underlying mechanism of fear generalization. We additionally investigated the role \\ of neuromodulators in basic olfactory fear learning through in vivo pharmacology, optogenetics, and \\ relative gene expression analysis. Manipulation of acetylcholine in the $\mathrm{OB}$ during olfactory fear learning \\ established cholinergic neurotransmission can enhance the strength of learned fear and that signaling \\ through muscarinic receptors is required for the formation of olfactory fear. Gene expression analysis \\ revealed that several neurotransmitter receptors are downregulated 4 hours following odor-shock pairing. \\ The majority of the downregulated genes were associated with $O B$ inhibition suggesting that fear \\ learning, behavioral fear generalization, and altered OB coding may arise from decreased inhibition in the \\ $\mathrm{OB}$. Altogether these results characterize neural correlates of fear generalization in the olfactory bulb at \\ both the population and single cell level and demonstrate the importance of neuromodulation in fear
}


learning, supporting the idea that fear generalization may initially arise from altered processing of incoming information in sensory areas. These results also highlight the importance of investigating the mechanisms of learning-induced sensory processing alterations as they relate to behavior in understanding fear generalization. This will bring insights into basic processes of learning and has the immense potential to translate to the treatment of disorders of fear generalization.

\section{Document Type}

Dissertation

Degree Name

Doctor of Philosophy (PhD)

\section{Program}

Biomedical Sciences

\section{Research Advisor}

Max L. Fletcher, Ph.D.

\section{Keywords}

calcium imaging, fear learning, generalization, neuromodulation, olfactory bulb

\section{Subject Categories}

Medicine and Health Sciences | Neurosciences 
Fear Learning and the Olfactory Bulb: Neural Correlates of Behavioral Fear Generalization

\author{
A Dissertation \\ Presented for \\ The Graduate Studies Council \\ The University of Tennessee \\ Health Science Center
}

In Partial Fulfillment

Of the Requirements for the Degree

Doctor of Philosophy

From The University of Tennessee

By

Jordan Marie Ross

August 2019 
Chapter 1 (C) 2018 by Springer Nature.

Chapter 2 (C) 2018 by Jordan Marie Ross and Max Fletcher. All other material (C) 2019 by Jordan Marie Ross.

All rights reserved. 


\section{DEDICATION}

Much like my dissertation, this dedication has six parts:

For my mother who raised me to keep going when things are hard and who brags about me proudly (though she has very little idea what I'm doing),

For Brittney whose unwavering words of support and encouragement guided me,

For Steve and Ryan, without whom I would never have gotten here,

For my friends who have become family, because of you my life outside of science has been rich and full,

For my raspberry for making life so sweet when it sometimes felt so sour, And for you - for at least reading as far as the dedication page. 


\section{ACKNOWLEDGEMENTS}

Getting a Ph.D. is both wonderful and awful. The process can build you up, but it can just as easily, probably more easily, tear you down. In a moment, it can make you question everything you thought you knew, who you are, what you're doing, if you're cut out for any of it. Research is a marathon of perseverance. I'm lucky to have found people to help me complete the marathon - and to help me complete literal marathons (half marathons anyway).

It takes a lot to get a Ph.D. and there are so many unsung heroes along the way but now is the chance to sing their praises (bear with me):

Max, simply put, thank you. Thank you for putting up with my crazy ideas and sass. Thank you for creating an environment where people can be scientifically productive and still have a rich life outside of the lab. Thank you for reminding us that sometimes you've done all you can do, and that's more than ok. Thank you for being the kind of PI whose first response in a crisis is, "are you ok?" Most importantly, thank you for being a friend.

A Ph.D. couldn't happen without a wonderful scientific environment to grow in so thank you to the members of my committee, Matt, John, Detlef, and Rob, for all that you've done. Thank you also to Bob and Bill and Reese for continually letting me TA. And thank you to every professor in the department for making UT the kind of place where lowly grad students can actually hang out and have fun with faculty members.

To Cameron, Mounir, and Stephanie, thank you for making sure I was never too productive at work.

Thank you to my Grad Girls (Cameron, Stephanie, Sarah, and Kate) for every Margarita Monday, every pub poll, every festival and concert, every paint night, every Raiford's/Molly's/Silky's/Peabody rooftop debauchery, and everything else in between. Because of you, Memphis became home.

Chaela and Beckett Presley, I am so grateful that you always invited me into your family - from children's birthday parties to Easters. I watched Beckett grow as I watched my dissertation grow and those two years with him will always hold a special place in my heart.

To my Mom, thank you for being a good example of working hard, especially when the work is hard. I couldn't be here without all of the sacrifices you've made. Thank you for my annual Valentine's Day cards and Easter basket because no matter how old or educated I become; I'll always be your kiddo.

Brittney, you definitely have a way with words and with timing. It always seemed when I was particularly bogged down with work or wallowing in scientific misfortune 
you would unknowingly send the perfect message to get my spirit back up. The words "cousin" and "sister" don't even begin to describe our bond; "best friend" might get close.

I couldn't thank my family without also acknowledging the Oggs. You all brought me in and treated me like one of your own. You became a family away from family and will be no matter where I go from here. I'm so lucky to have had you all in my life.

There are so many others that have helped along the way that I can't even give all the names. You know who you are. Thank you for sharing every moment of this experience with me. Thank you for reading all my tedious writing, including this Acknowledgements section.

I never would have guessed six years ago I'd have this many people in my life I wanted to thank for their support and guidance. I would like to think that is the best measuring stick for what I've accomplished during my Ph.D. 


\begin{abstract}
The exploration of how learning alters neural coding to guide behaviors remains fundamental to neuroscience. At the most basic level, the ability for organisms to flexibly adapt to changing environments and situations is paramount to biological success and often manifests in behavioral responses controlled by neural activity. For example, organisms must modify their behavior to defensive responses in the face of biological threat. Neural circuitry is involved in coordinating an initial defensive behavioral response but must undergo reorganization in order to reliably employ defensive responses in subsequent encounters based on a cue that signals imminent danger. One such form of this learning is associative fear learning, in which an organism learns to associate an initially neutral stimulus, which by itself has no biological relevance, with an innately fear-inducing stimulus. After temporally pairing the two stimuli, organisms learn that the initially neutral stimulus predicts the fear-inducing stimulus such that encountering the former induces similar behavioral responses as the latter.
\end{abstract}

Interestingly, associative fear learning is often not specific and organisms display fear responses to completely neutral stimuli that have never been associated with the innately fear-inducing stimulus. This aberrant expression of fear is referred to as generalization and is a hallmark of many psychological disorders such as anxiety and post-traumatic stress disorder. The underlying cause of fear generalization remains unknown; however, there are two predominant theories. One theory is fear generalization arises as a better-safe-than-sorry strategy, wherein sensory information remains perceptually segregated between fear predictive and neutral stimuli but the organism responds to both the same way despite the perceptual information. The second theory argues that learning alters sensory processing to make the stimuli more difficult to perceptually distinguish, which cascades into behaviorally treating them as the same.

The olfactory bulb (OB) represents an ideal model system for studying the extent to which fear learning alters sensory coding in ways that support failure of perceptual discrimination due to its odotopic organization that creates unique "maps" of odor representation for each experience odor. Therefore, we used both wide-field and 2-photon (2P) calcium imaging of the OBs of awake mice in combination with classical olfactory fear conditioning to investigate how learning changes sensory representation of the conditioned odor, the initially neutral odor specifically paired with fear-inducing foot shock, as well as neutral odors never paired with shock. This allowed direct testing of the extent to which fear learning reorganizes olfactory processing in a manner that supports the failure to discriminate hypothesis of fear generalization.

Both wide-field and 2P imaging revealed enhanced odor-evoked responses following fear learning that likely signal increased salience of incoming sensory information. Furthermore, the responses evoked by neutral odors became more similar to those evoked by the conditioned odor at both the population level (wide-field) and in a specific subset of OB output cells (2P), indicating neutral odors were more difficult to distinguish from the conditioned odor. Importantly, the enhanced odor-evoked responses 
were not attributable to behavioral state change nor top-down influence from the amygdala, the area widely believed to be involved in aberrant fear. Together, this evidence supported the failure of perceptual discrimination at early stages of sensory process as the underlying mechanism of fear generalization.

We additionally investigated the role of neuromodulators in basic olfactory fear learning through in vivo pharmacology, optogenetics, and relative gene expression analysis. Manipulation of acetylcholine in the OB during olfactory fear learning established cholinergic neurotransmission can enhance the strength of learned fear and that signaling through muscarinic receptors is required for the formation of olfactory fear. Gene expression analysis revealed that several neurotransmitter receptors are downregulated 4 hours following odor-shock pairing. The majority of the downregulated genes were associated with OB inhibition suggesting that fear learning, behavioral fear generalization, and altered $\mathrm{OB}$ coding may arise from decreased inhibition in the OB.

Altogether these results characterize neural correlates of fear generalization in the olfactory bulb at both the population and single cell level and demonstrate the importance of neuromodulation in fear learning, supporting the idea that fear generalization may initially arise from altered processing of incoming information in sensory areas. These results also highlight the importance of investigating the mechanisms of learning-induced sensory processing alterations as they relate to behavior in understanding fear generalization. This will bring insights into basic processes of learning and has the immense potential to translate to the treatment of disorders of fear generalization. 


\title{
TABLE OF CONTENTS
}

\author{
CHAPTER 1. AVERSIVE LEARNING-INDUCED PLASTICITY \\ THROUGHOUT THE ADULT MAMMALIAN OLFACTORY SYSTEM:

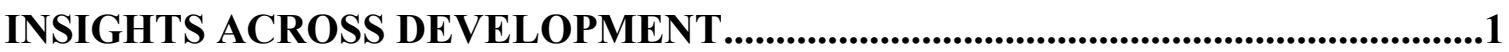

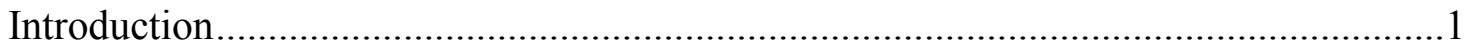 \\ Adult Olfactory Fear Conditioning Paradigms ......................................................

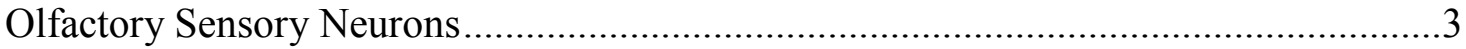

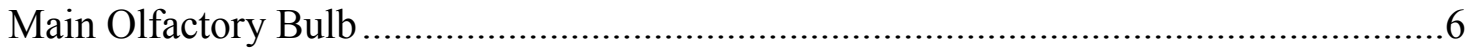

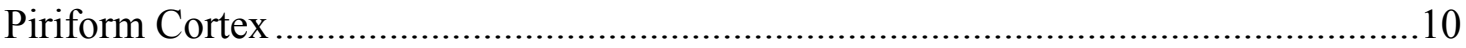

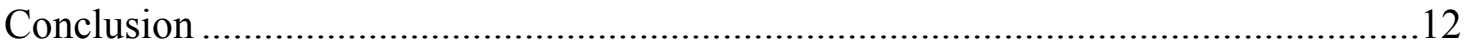

\section{CHAPTER 2. LEARNING-DEPENDENT AND -INDEPENDENT ENHANCEMENT OF MITRAL/TUFTED CELL GLOMERULAR ODOR RESPONSES FOLLOWING OLFACTORY FEAR CONDITIONING IN}

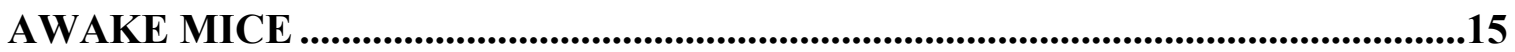

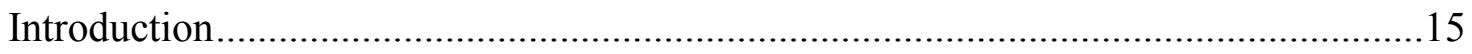

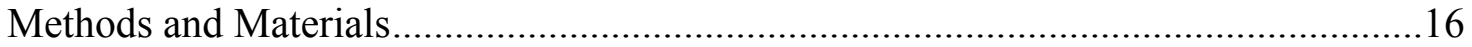

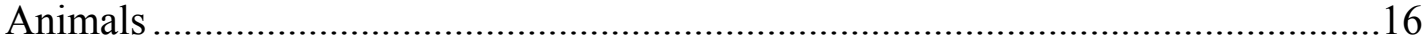

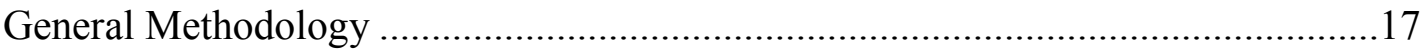

Surgical procedures..................................................................................... 17

Drug infusions and placement verification ......................................................... 17

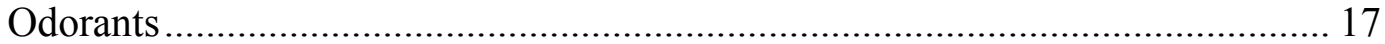

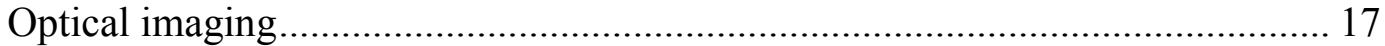

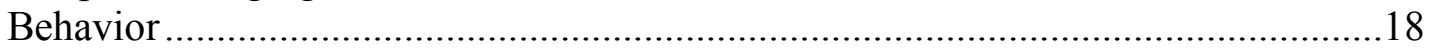

Olfactory fear conditioning............................................................................ 18

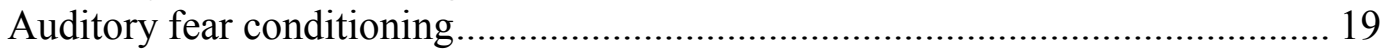

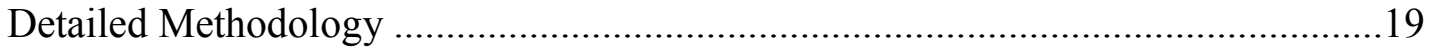

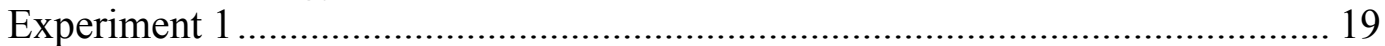

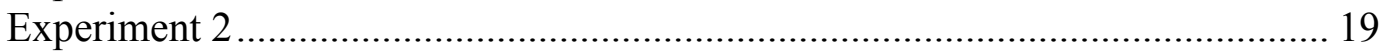

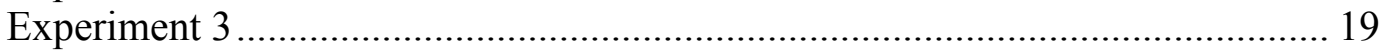

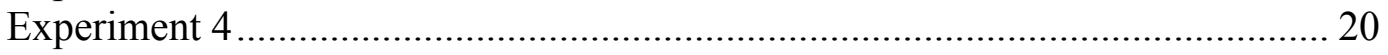

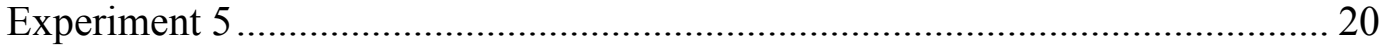

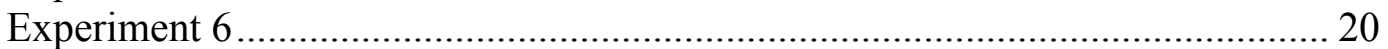

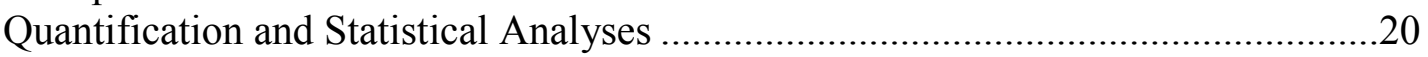

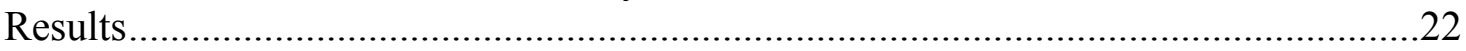

Olfactory Aversive Conditioning Produces Fear Generalization to Multiple

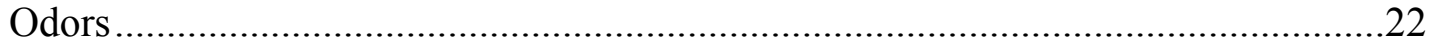

Olfactory Aversive Conditioning Potentiates Glomerular Responses in Awake

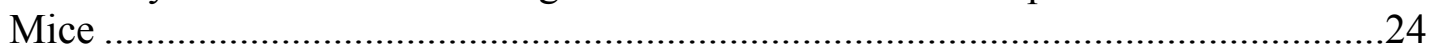

Olfactory Aversive Conditioning Does Not Alter Sniffing Rates in Awake,

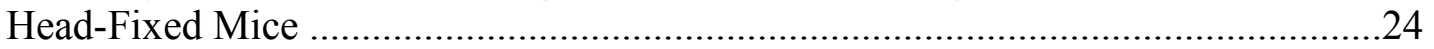

Olfactory Aversive Conditioning Non-Specifically Potentiates Glomerular Responses to All Odors in Awake Mice .28 
Post-Training Enhancement of Neutral Odor Responses Is Independent of CS

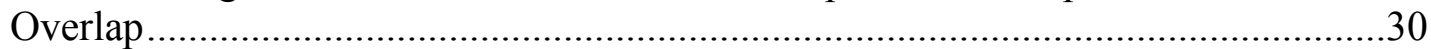

Olfactory Fear Conditioning Increases Odor Representation Similarity Between

CS and Neutral Odors

Olfactory Fear Learning Induces Long-Lasting Behavioral Fear and Enhanced

Glomerular Responses

Anesthetized Mice Display Weaker Glomerular Enhancements and Suppression

Following Olfactory Fear Learning ....

Post-Training Glomerular Response Enhancements Are Independent of General

Fear States

Global, but Not CS-Specific, Glomerular Enhancements Are Fear Learning

Dependent

Suppressing Fear Centers During Expression Does Not Suppress Learning-

Induced Glomerular Enhancements .

Discussion

Potential Distinct Mechanisms of Experiment-Induced Glomerular Plasticity .........43

Potential Impact

\section{CHAPTER 3. CHOLINERGIC MODULATION OF OLFACTORY ASSOCIATIVE AVERSIVE MEMORY AND OLFACTORY BULB PLASTICITY}

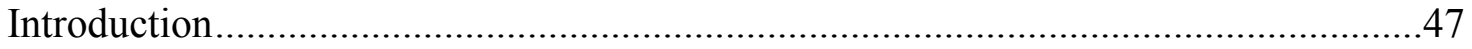

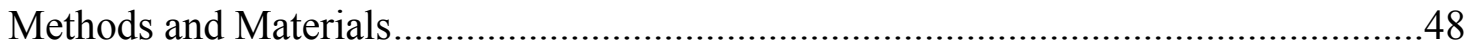

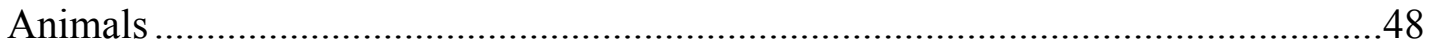

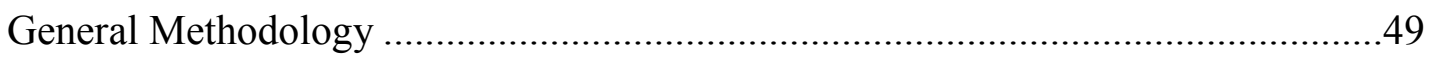

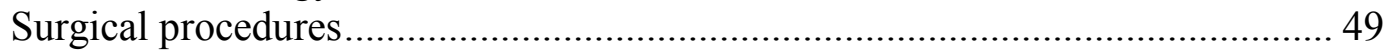

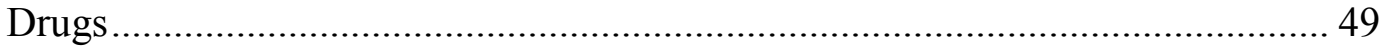

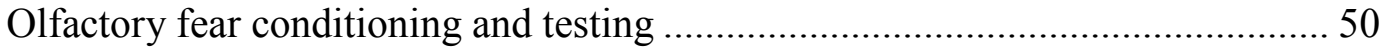

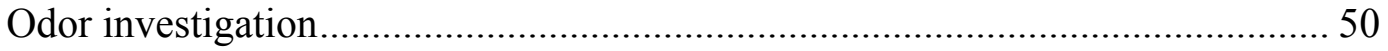

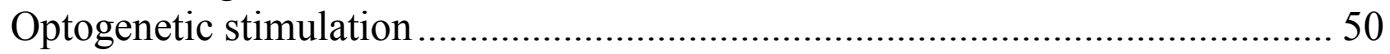

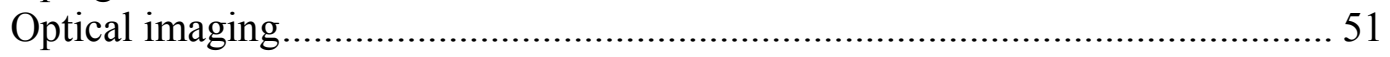

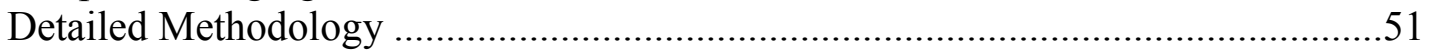

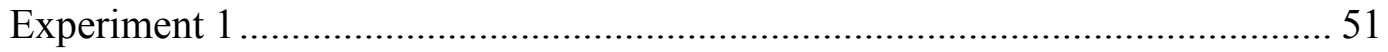

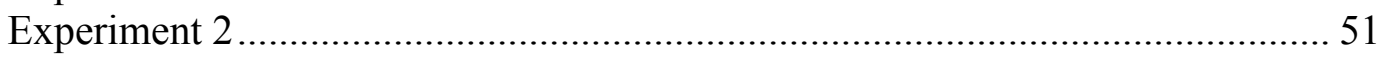

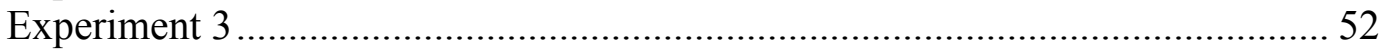

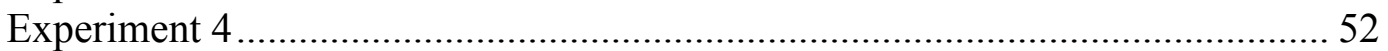

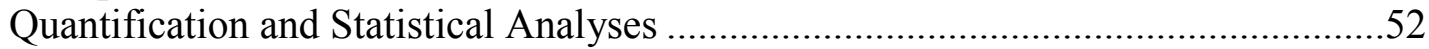

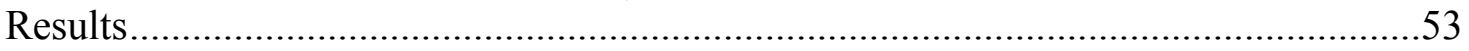

Muscarinic Neurotransmission Is Required in the OB During Acquisition for

Olfactory Fear Learning ...............................................................................5

OB Muscarinic Neurotransmission Is Not Required for Expression of Olfactory

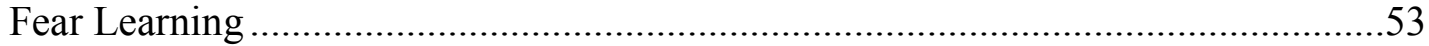

OB Muscarinic Neurotransmission, Specifically Through mAChR1, Is Required for Olfactory Fear Learning ....................................................................................55

Stimulating Release of OB ACh During Odor-Shock Pairing Strengthens

Olfactory Fear Learning 
Olfactory Investigative Behavior Is Not Affected by Direct OB Antagonism of

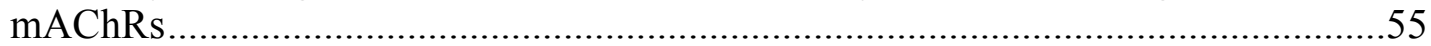

Muscarinic Signaling Is Required for Olfactory Fear Learning-Induced

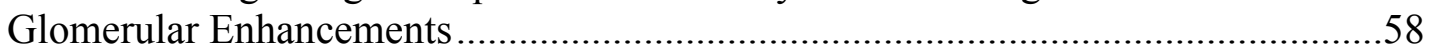

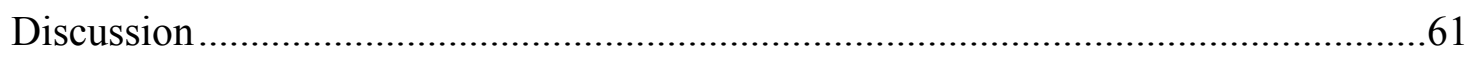

\section{CHAPTER 4. OLFACTORY FEAR CONDITIONING INDUCES ROBUST REORGANIZATION OF SALIENCE AND IDENTITY-CODING IN OLFACOTRY BULB OUTPUT CELLS ...............................................................64}

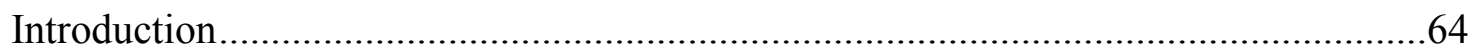

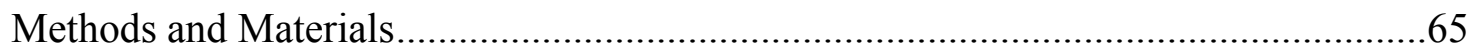

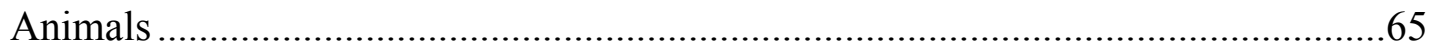

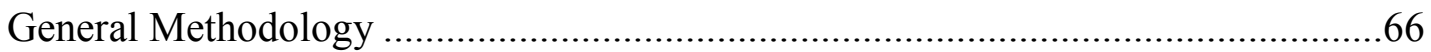

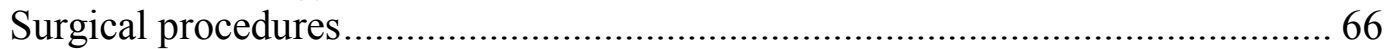

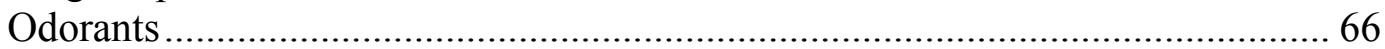

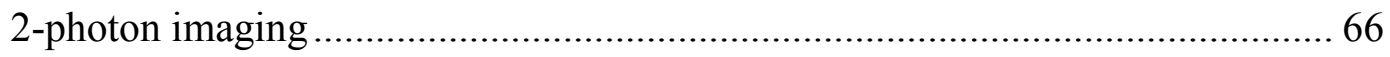

Olfactory Fear Conditioning and Testing ............................................................68

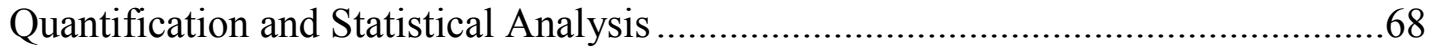

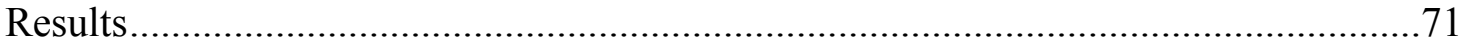

Olfactory Aversive Conditioning Induces Behavioral Generalization Across

Structurally Similar Odorants ................................................................................

Olfactory Aversive Conditioning Enhances Odor-Evoked Responses in Superficial Tufted Cells .....................................................................................

Enhanced Superficial Tufted Cells Responses Do No Alter Coding of Odor

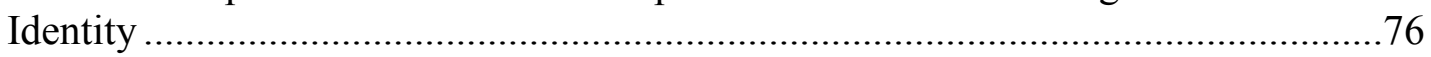

Mitral Cell Odor Responses Are Enhanced Following Olfactory Aversive

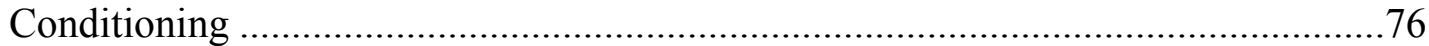

Olfactory Fear Conditioning Increases Odor-Evoked Mitral Cell Ensemble

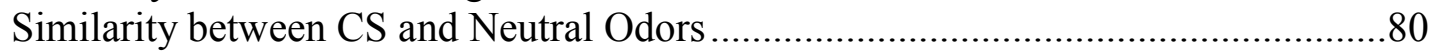

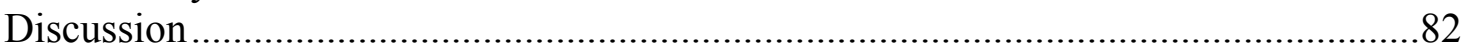

Olfactory Fear Learning Changes Odor Coding in Output Cells in Distinct Ways...82

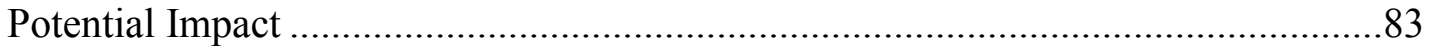

\section{CHAPTER 5. OLFACTORY FEAR CONDITIONING-INDUCED REGULATION OF NEUROTRANSMITTER RECEPTOR GENE} EXPRESSION IN THE OLFACTORY BULB........................................................85

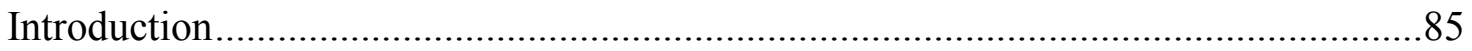

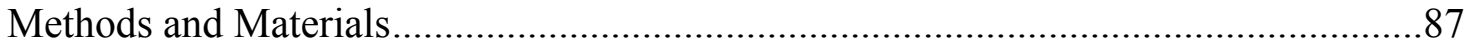

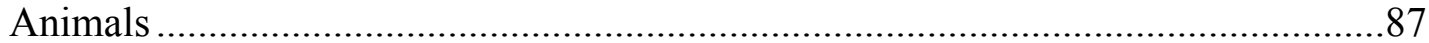

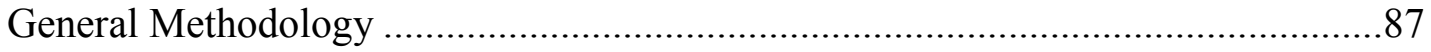

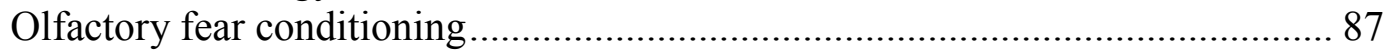

Olfactory bulb tissue collection and preparation ................................................. 87

Real time-polymerase chain reaction array......................................................... 88

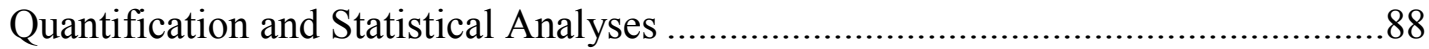

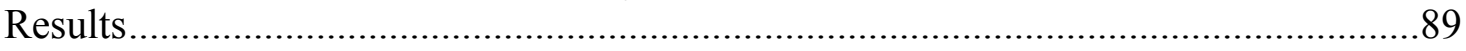


Odor-Shock Pairing Significantly Modifies Gene Expression 4 Hours After

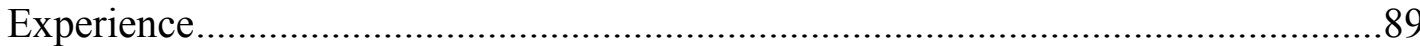

Twenty-Four Hours After Odor-Shock Pairing, Gene Expression Changes Are

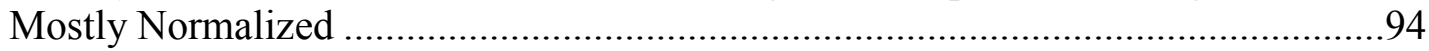

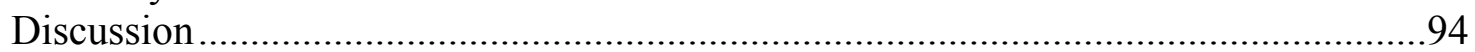

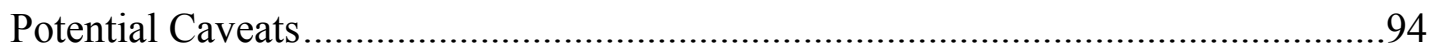

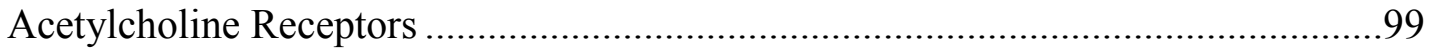

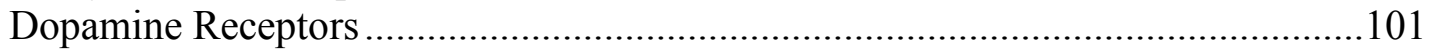

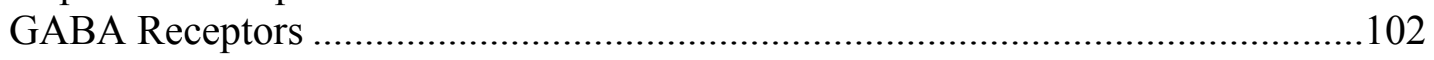

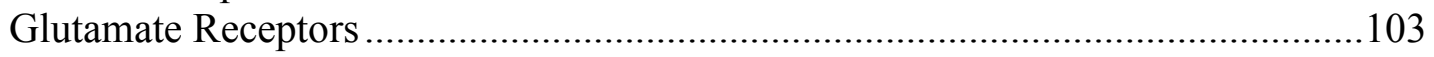

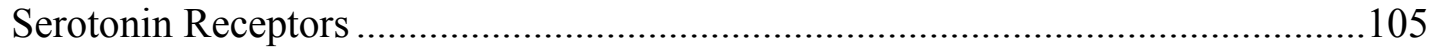

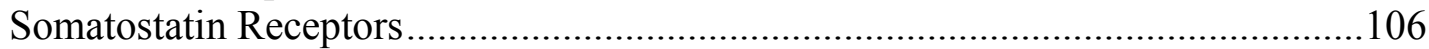

Olfactory Fear Conditioning-Induced Alterations of Neuromodulation ..................107

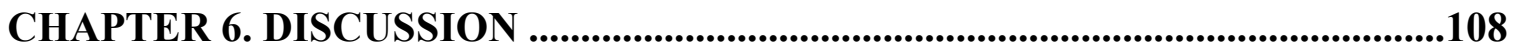

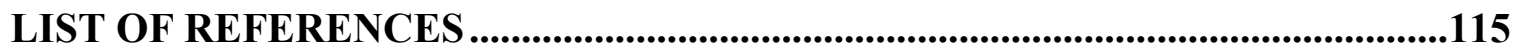

VITA 


\section{LIST OF TABLES}

Table 5-1. Differences in relative OB gene expression between Control and Paired animals 4 hours after training................................................................. 90

Table 5-2. Differences in relative OB gene expression between Control and Paired animals 24 hours after training. 


\section{LIST OF FIGURES}

Figure 1-1. Simplified schematic of mammalian olfactory system circuitry.

Figure 2-1. Olfactory aversive conditioning results in robust olfactory fear and generalization.

Figure 2-2. Olfactory aversive conditioning enhances glomerular responses.

Figure 2-3. Post-training enhancements are independent of glomerular response amplitude. .26

Figure 2-4. Mean instantaneous respiration frequence (MIF) is stable. .27

Figure 2-5. Enhanced responses are global, not odor- or glomerular-specific.

Figure 2-6. Olfactory fear learning induces long-lasting behavioral fear and glomerular enhancements.

Figure 2-7. Learning-induced glomerular changes are variable in anesthetized mice. ...34

Figure 2-8. The expression of learning-induced glomerular response enhancements is independent of general fear states.

Figure 2-9. Generalized, but not CS-specific, glomerular enhancements are associative learning-dependent.

Figure 2-10. Amygdala inactivation during expression of learning does not impact glomerular responses.

Figure 3-1. Direct OB application of scopolamine during fear conditioning impairs olfactory aversive fear learning but has no effect on expression of previously learned fear.

Figure 3-2. Inhibition of $\mathrm{mAChR} 1$, but not $\mathrm{mAChR} 2$, decreases behavioral freezing to the conditioned odor.

Figure 3-3. Enhanced OB acetylcholine during odor-shock pairing augments olfactory fear learning.

Figure 3-4. Antagonism of $\mathrm{OB} \mathrm{mAChRs}$ does not alter olfactory driven behaviors. .....59

Figure 3-5. Activation of $\mathrm{mAChRs}$ are necessary for odor-shock driven OB glomerular enhancements.

Figure 4-1. Multi-day imaging of single cells in olfactory bulbs of awake mice.

Figure 4-2. Mitral cells exhibit varied response profiles. . .70 
Figure 4-3. Fear conditioning leads to behavioral fear generalization.

Figure 4-4. Olfactory fear learning globally increases salience coding in olfactory bulb superficial tufted cells.

Figure 4-5. Fear learning does not alter odor identity coding in superficial tufted cells

Figure 4-6. Mitral cells demonstrate enhanced salience coding following olfactory fear conditioning.

Figure 4-7. Neutral odor-evoked responses become more similar to conditioned odor-evoked responses in mitral cells.

Figure 5-1. Relative OB fold change of all neurotransmitter receptor genes in Paired mice compared to Controls 4 hours after olfactory fear conditioning.

Figure 5-2. Paired experience OB gene expression of regulated genes 4 hours after olfactory fear conditioning.

Figure 5-3. Relative OB fold change of all neurotransmitter receptor genes in Paired mice compared to Controls 24 hours after olfactory fear conditioning. ......97

Figure 5-4. Paired-experience OB gene expression of regulated genes 24 hours after olfactory fear conditioning. 


\section{LIST OF ABBREVIATIONS}

\begin{tabular}{|c|c|}
\hline$\Delta$ & Change \\
\hline ac & Amygdalar capsule \\
\hline $\mathrm{ACh}$ & Acetylcholine \\
\hline $\mathrm{AChR}$ & Acetylcholine receptor \\
\hline AFDX & AF-DX 116 \\
\hline AMPA & $\alpha$-amino-3-hydroxy-5-methyl-4-isoxazolepropionic acid \\
\hline AMPAR & AMPA receptor \\
\hline ANOVA & Analysis of variance \\
\hline AP & Anteroposterior \\
\hline $\mathrm{aPCx}$ & Anterior piriform cortex \\
\hline BDNF & Brain derived neurotrophic factor \\
\hline BLA & Basolateral amygdala \\
\hline BZ & Benzaldehyde \\
\hline CaMKII & $\mathrm{Ca} 2+/$ calmodulin-dependent protein kinase II \\
\hline cAMP & Cyclic adenosine monophosphate \\
\hline cDNA & Complimentary DNA \\
\hline $\mathrm{CoA}$ & Cortical nucleus of the amygdala \\
\hline CREB & cAMP response element-binding protein \\
\hline $\mathrm{CS}$ & Conditioned stimulus \\
\hline $\mathrm{CS}+$ & Conditioned stimulus (reinforced) \\
\hline CS- & Conditioned stimulus (unreinforced) \\
\hline $\mathrm{Ct}$ & Cycle threshold \\
\hline
\end{tabular}




\begin{tabular}{|c|c|}
\hline dB & Decibel \\
\hline DMSO & Dimethyl sulfoxide \\
\hline DV & Dorsoventral \\
\hline $\mathrm{E} 4$ & Ethyl butyrate \\
\hline E5 & Ethyl valerate \\
\hline E6 & Ethyl hexanoate \\
\hline ec & External capsule \\
\hline EEG & Electroencephalogram \\
\hline EPL & External plexiform layer \\
\hline EPSP & Excitatory postsynaptic potential \\
\hline GABA & Gamma-Aminobutyric acid \\
\hline GC & Granule cell \\
\hline GCaMP & GFP-calmodulin-M13 peptide fusion protein \\
\hline GCL & Granule cell layer \\
\hline GFP & Green fluorescent protein \\
\hline GL & Glomerular layer \\
\hline HKG & Housekeeping gene \\
\hline $\mathrm{Hz}$ & Hertz \\
\hline i.p. & Intraperitoneal \\
\hline IPL & Internal plexiform layer \\
\hline ITI & Inter-trial interval \\
\hline JGC & Juxtaglomerular cell \\
\hline KAR & Kainate receptor \\
\hline
\end{tabular}




\begin{tabular}{|c|c|}
\hline $\mathrm{kHz}$ & Kilohertz \\
\hline LED & Light emitting diode \\
\hline LTP & Long-term potentiation \\
\hline $\mathrm{M} / \mathrm{T}$ & Mitral/Tufted \\
\hline $\mathrm{mA}$ & Milliamps \\
\hline $\mathrm{mAChR}$ & Muscarinic acetylcholine receptor \\
\hline $\mathrm{MC}$ & Mitral cell \\
\hline MCL & Mitral cell layer \\
\hline $\mathrm{MeA}$ & Medial nucleus of the amygdala \\
\hline mGluR & Metabotropic glutamate receptor \\
\hline MIF & Mean instantaneous (respiratory) frequency \\
\hline ML & Mediolateral \\
\hline mRNA & Messenger RNA \\
\hline MTC & Mitral/tufted cell \\
\hline MUSC & Muscimol \\
\hline nAChR & Nicotinic acetylcholine receptor \\
\hline $\mathrm{NE}$ & Norepinephrine \\
\hline NMDA & N-methyl-D-aspartate \\
\hline NMDAR & NMDA receptor \\
\hline OB & Olfactory bulb \\
\hline ONL & Olfactory nerve layer \\
\hline OSN & Olfactory sensory neuron \\
\hline pCREB & Phosphorylated CREB \\
\hline
\end{tabular}




\begin{tabular}{|c|c|}
\hline $\mathrm{PCx}$ & Piriform cortex \\
\hline PGC & Periglomerular cell \\
\hline PIR & Pirenzipine dihydrochloride \\
\hline pPCx & Posterior piriform cortex \\
\hline $\mathrm{ppm}$ & Parts per million \\
\hline RLI & Resting light intensity \\
\hline RMS & Root mean square \\
\hline RNA & Ribonucleic acid \\
\hline RT-PCR & Real-time polymerase chain reaction \\
\hline s.c. & Subcutaneous \\
\hline SAC & Short-axon cell \\
\hline SCOP & Scopolamine hydrobromide \\
\hline SD & Standard deviation \\
\hline SEM & Standard error of the mean \\
\hline SSTR & Somatostatin receptor \\
\hline STC & Superficial tufted cell \\
\hline s.v. & Saturated vapor \\
\hline VEH & Vehicle \\
\hline 2-DG & 2-deoxyglucose \\
\hline $2 \mathrm{H}$ & 2-heptanone \\
\hline $2 \mathrm{P}$ & 2-photon imaging \\
\hline 5 -HT & 5-hydroxytryptamine (serotonin) \\
\hline
\end{tabular}




\section{CHAPTER 1. AVERSIVE LEARNING-INDUCED PLASTICITY THROUGHOUT THE ADULT MAMMALIAN OLFACTORY SYSTEM: INSIGHTS ACROSS DEVELOPMENT ${ }^{1}$}

\section{Introduction}

In order to survive, organisms must be able to modify their behaviors in response to experience. Such flexibility requires modification of neural properties, circuitry, and connectivity that can be long-lasting, a process referred to as neural plasticity. Neuroplasticity can take many forms from subcellular changes in intrinsic neuronal properties or receptor expression to large-scale changes in coding and neural networks (Amtul and Atta Ur 2015; Carasatorre and Ramirez-Amaya 2013; Foehring and Lorenzon 1999). Experiences, such as associative learning, are known to induce plasticity in several brain areas, which ultimately support altered behavioral responses (Kolb and Gibb 2014; Sweatt 2016). Aversive, or fear, conditioning involves learning to associate some initially neutral, conditioned stimulus (CS) with an aversive unconditioned stimulus, such that subsequent presentations of the CS produce a measurable aversive or avoidance behavior (Pavlov 1927). Aversive learning in mammals is associated with neural plasticity in areas such as the hippocampus and frontal cortex, which are also involved in appetitive conditioning (Broersen 2000); however, aversive learning additionally induces plasticity in areas specific to fear, such as the amygdala (Izquierdo et al. 2016; Maren 2001). Furthermore, aversive conditioning alters coding in sensory cortices that are more specific to the type of stimulus used for conditioning. For example, pairing an auditory cue with an aversive electric shock leads to altered processing of the CS in auditory cortex (Grosso et al. 2015; Weinberger 1998).

Olfactory aversive learning induces changes in sensory coding in the adult mammalian olfactory pathway, a fact we have known for several decades (Bressler 1988; Coopersmith et al. 1986; Freeman and Schneider 1982; Pager and Royet 1976), yet we have barely begun to scratch the surface as to the mechanisms underlying neural plasticity responsible for such changes. In recent years, altered coding has been reported across all stages of the adult olfactory pathway including olfactory sensory neurons (OSN) (Kass et al. 2013), olfactory bulb (OB) cells (Fletcher 2012; Kass and McGann 2017; Ross and Fletcher 2018), and piriform cortex (PCx) cells (Chen et al. 2011; Sevelinges et al. 2004); however, extensive investigation of electrophysiological or molecular mechanisms or widespread network alterations underlying the observed coding changes is still lacking in adults. Instead, much of the work regarding mechanisms of olfactory associative learning comes from neonates; however, most of this information regarding odor learning in pups comes from a time point before which the brain or olfactory system is fully developed. While the majority of cell types and all types of

\footnotetext{
${ }^{1}$ Reprinted from final submission by permission from Springer Nature, Journal of Bioegenergetics and Biomembranes. Aversive learning-induced plasticity throughout the adult mammalian olfactory system: Insights across development, doi: 10.1007/s10863-018-9770-z, Ross, J. M. \& Fletcher, M. L C 2018
} 
synapses are present at birth, mammalian OB synapses continue to increase and mature well into adolescence (Treloar et al. 2010) and PCx pyramidal cells mature in the first postnatal weeks (Sarma et al. 2011). Importantly, olfactory aversive conditioning in pups prior to postnatal day 10 (P10) results in behavioral paradoxical preference of the conditioned odor, an acquired preference to the stimulus paired with an aversive outcome, which is a different behavioral outcome than adults and attributable to developmental differences (Roth et al. 2006).

For example during the first weeks of life, the intrinsic properties of mitral cells undergo significant changes (Almli et al. 1985; Yu et al. 2015) and most of the OB inhibitory interneurons seen in adults develop (Mair et al. 1982; Rosselli-Austin and Altman 1979). The local OB circuits evolve throughout the first month of life, giving rise to different oscillatory activity in both gamma and beta frequencies (Dietz et al. 2011; Fletcher et al. 2005), which may impact odor discrimination and learning (Kay 2014). Furthermore, centrifugal inputs to the OB, which play a pivotal role in olfactory processing and aversive fear learning, develop and mature postnatally (Le Jeune and Jourdan 1991; McLean and Shipley 1991; Rea and Nurnberger 1986; Schwob and Price 1984a,b; Wilson and Leon 1988). One of the biggest differences related to fear learning is the lack of amygdala plasticity in neonates, which results in paradoxical odor preference following aversive olfactory conditioning (Landers and Sullivan 2012). The lack of fully developed brains combined with differences in behavioral outcome has impeded the application of identified pup mechanisms to adult olfactory fear learning. While interest in characterizing coding changes as a result of adult olfactory aversive conditioning has surfaced in recent years, especially with the advent of in vivo calcium imaging, there is a paucity of data regarding mechanistic insight of the neural underpinnings.

Studies aiming to test whether the molecular mechanisms identified in pup conditioning, both aversive and associative, apply to adults will dramatically increase our understanding of adult aversive learning-induced sensory plasticity. In addition, studies that identify convergent mechanisms preserved through mammalian development may offer insights to other model systems, including insects and humans. Understanding the olfactory aversive learning-induced alterations in neural circuity and neurobiology in the mammalian olfactory system has the potential to reveal how the brain is able to coordinate behavior even at the earliest stages of encoding and consolidating salient sensory information. Therefore, in order to advance the understanding of aversive learning-induced neuroplasticity, we must review the findings from the different stages of development across the entire olfactory system, including those with different behavioral outcomes, to build testable hypotheses that will inform future studies of aversive learning-induced sensory plasticity.

\section{Adult Olfactory Fear Conditioning Paradigms}

Olfactory fear conditioning involves pairing an initially neutral odor with an aversive outcome, which commonly in adults is a mild foot shock. During this conditioning paradigm, animals learn to associate the neutral odor with the foot shock, 
such that subsequent presentations of the conditioned odor elicit a measurable behavioral fear response. One of the most common measures of behavioral fear is behavioral freezing, the complete absence of all non-breathing movements. While pairing odors with an aversive outcome during conditioning and measuring startle or freezing responses are common across most current studies involving adult mammalian fear learning, there are many variations in experimental paradigms such as aversive stimulus intensity and duration, number of pairings within a session, and conditioned odorant used. These discrepancies likely impact the magnitude of learning-induced effects but do not appear to greatly alter behavior or plasticity (Kass and McGann 2017; Kudo et al. 2004; Morrison et al. 2016; Pavesi et al. 2012; Ross and Fletcher 2018); however, there are a number of factors which might. One such parameter is whether other odors are presented during training. Standard classical conditioning involves presenting a single stimulus, which always coincides with an aversive outcome and typically results in generalized fear responses. In contrast, discriminant conditioning involves presenting at least two stimuli, one of which always coincides with an aversive outcome while the other never coincides. Unlike classical conditioning, discriminant conditioning is designed to produce fear that is specific to the stimulus predictive of the aversive event (Pavlov 1927), which represents an important difference that could influence learning-induced plasticity.

Additionally, the number of training sessions and timeline of experiments could impact the longevity of behavioral responses and plasticity. Traditionally, conditioning is performed in a single day with testing occurring the next day (Barnes et al. 2011; Chen et al. 2011; Curzon et al. 2009; Fletcher 2012; Lugo et al. 2014; Ross and Fletcher 2018; Weinberger 2007); however, some recent publications spread multiple training sessions over several days or weeks before assessing behavior and plasticity (Jones et al. 2008; Kass et al. 2013; Morrison et al. 2015), making it difficult to compare these results to those observed following more traditional single-day training paradigms. Multiple training sessions spread over time could possibly induce plasticity through separate or additional mechanisms than training which occurs in a single day. These functional differences are important to consider when designing experiments to evaluate fear learning-induced olfactory plasticity.

\section{Olfactory Sensory Neurons}

The OSNs represent the initial site of olfactory processing (Figure 1-1). Residing in the olfactory epithelium, each OSN expresses a single receptor, which recognizes a specific structural feature of odor molecules (Buck and Axel 1991). When odor

molecules bind to OSNs, information regarding the bound odorant travels along the OSN axons expressing the same receptor into the $\mathrm{OB}$ where they synapse on a specific target glomerulus in the OB (Ressler et al. 1994; Sullivan et al. 1995). This initial stage of odorant processing experiences abundant reorganization, especially during development, that refines the number of OSNs in the epithelium and axons that synapse in the OB (Marcucci et al. 2011). Given the highly plastic nature of OSNs, they present an interesting area of investigation regarding olfactory aversive learning-induced plasticity 


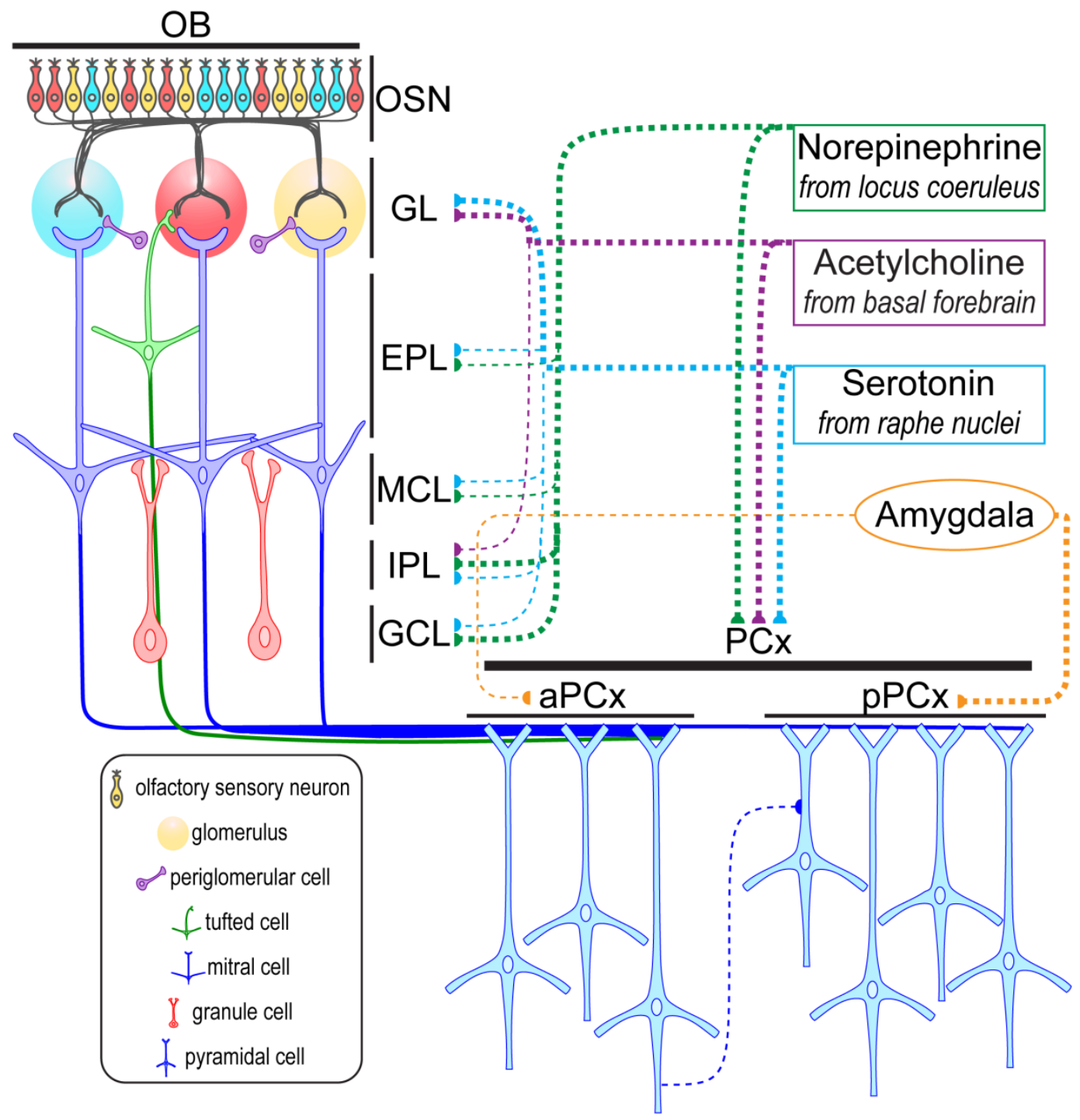

Figure 1-1. Simplified schematic of mammalian olfactory system circuitry. As described in the text, olfactory sensory neurons (OSNs) express a single receptor, and all those expressing the same receptor converge to synapse onto a specific glomerulus. Within the glomerulus, the axons of OSNs synapse onto various olfactory bulb (OB) cell types, including mitral cells, tufted cells, and periglomerular cells. Granule cells provide a major source of inhibition onto mitral cells (depicted) and tufted cells. Both mitral and tufted cells project to the piriform cortex $(\mathrm{PCx})$ where they synapse primarily on $\mathrm{PCx}$ pyramidal cells. Mitral and tufted cells project densely to anterior PCx, whereas only mitral cells project to posterior PCx. The posterior PCx also receives projections from anterior PCx. Finally, the amygdala projects to both anterior and posterior PCx, though with differing density as represented by line thickness. The olfactory system receives centrifugal neuromodulatory input from several regions. The known norepinephrine, acetylcholine, and serotonin projections to both $\mathrm{OB}$ and $\mathrm{PCx}$ are depicted, with the strength of innervation illustrated by line thickness. Information regarding OB neuromodulatory input is broken into OB layers. A key with depictions of each represented cell type is depicted in the bottom left hand corner. 
in pups as well as adults. Importantly, restructuring at this level could be fairly slow. In pups OSN plasticity involves altered rates of pruning to refine olfactory signals or survival of OSNs that would be marked for pruning, which may also serve as an adult substrate for learning-induced plasticity (Alonso et al. 2006; Graziadei and Graziadei 1979; Mackay-Sim and Kittel 1991). Another possible source of adults OSN plasticity would require neurogenesis and functional integration of new neurons (Carleton et al. 2002; Cheetham et al. 2016).

In adults, previous work (Jones et al. 2008) using multi-day aversive classical olfactory conditioning, in which a single odorant, acetophenone, was paired with foot shock, induced fear responses specific to the conditioned stimulus. Furthermore, both the number of acetophenone-responsive OSNs and the size of the corresponding acetophenone-responsive glomerulus increased following acetophenone-shock conditioning but not when shock was paired with a different odor, suggesting odorspecific glomerular changes at the OSN level. Extinction training reverses both the increased number of acetophenone-responsive OSNs as well as the size of the corresponding glomerulus (Morrison et al. 2015). While the exact cause of odor-specific changes was not directly studied, the authors' hypothesized increased survival of OSNs that would normally turnover might lead to increased OSN number and size of the presynaptic component of the corresponding glomerulus, possibly due to OB release of pro-survival neurotrophic factors. This idea is in line with previous work from the same group, demonstrating upregulation of the brain-derived neurotrophic factor (BDNF) gene in the OB following odor-shock conditioning (Jones et al. 2007). However, upregulation of OB BDNF was found following aversive learning as well as passive odor exposure, whereas passive exposure did not increase glomerular size (Jones et al. 2008; Jones et al. 2007). Altogether, this makes upregulation of BDNF alone an unlikely source for increased glomerular size following aversive learning. In addition, upregulation of a ligand, such as BDNF, is unlikely to increase odorant-specific OSN survival as it would be more likely to promote overall survival of all OSNs, though perhaps increased levels of BDNF along with concomitant upregulation of receptor at target OSNs or signaling cascades downstream of BDNF itself could explain odor-specific OSN alterations (for review see Cowansage et al. 2010).

A recent neuroimaging paper used synaptopHluorin, a $\mathrm{pH}$-sensitive form of GFP as an indicator of neurotransmitter release, to measures changes in OSN coding in adult anesthetized mice before and after discriminant olfactory fear learning (Kass et al. 2013). Discriminant conditioning differs from classical conditioning in that a single stimulus is paired with an aversive outcome (CS+) but at least one other stimulus (CS-) is presented in the absence of shocks. This type of paradigm produces fear learning more specific to the $\mathrm{CS}+$. When presented the $\mathrm{CS}+$ following conditioning, OSNs exhibited enhanced responses; however, the enhanced responses were specific to the $\mathrm{CS}+$, as there was no enhancement of OSNs responsive to a second, unpaired stimulus (CS-) following olfactory fear conditioning. The hypothesized mechanism of enhanced OSN responses was increased sensitivity of OSNs to subsequent presentations of the conditioned odor following training; however, an exact mechanism underlying increased sensitivity was 
not identified (Kass et al. 2013). Importantly, the discriminant paradigm in which mice are taught to fear a specific odor could provide a basis for odorant-specific coding changes different from that seen in classical fear conditioning studies. Repeating these experiments in a classical conditioning task could shed light on whether these different learning paradigms with different behavioral outcomes result in the same forms of plasticity.

These studies suggest aversive learning alters OSN input to OB glomeruli, which has also been observed in pups following olfactory conditioning (Kerr and Belluscio 2006); however, all of these studies exploring learning-induced plasticity to OSNs employed multiple training sessions carried out over the course of days or weeks. This overtraining model would allow for massive structural reorganization in the $\mathrm{OB}$ that likely cannot occur in single-day training paradigms where changes are still visible within 24 hours and likely, therefore, represent a completely different plastic mechanism. While OSNs certainly contribute to olfactory processing and each of these studies indicates that overtraining can alter OSN input to the OB, it is still uncertain whether coding changes or structural plasticity occurs at the level of OSNs in pups or adults when using more ecologically valid single-day aversive training paradigms.

\section{Main Olfactory Bulb}

The main OB represents the first site of olfactory processing in the central nervous system (Figure 1-1). It receives input from the OSNs into neuropil structures called glomeruli comprised of the dendrites of OB output (mitral and tufted) cells (Shipley and Ennis 1996). At the glomerular layer, odor information is transformed into a highly-organized spatial map that forms initial odor identity (Bozza et al. 2004; Fletcher et al. 2009; Mori et al. 2006; Spors and Grinvald 2002; Storace and Cohen 2017; Wachowiak and Cohen 2001). After receiving information regarding bound odorants from the OSNs, output cells project that information to other olfactory centers, including PCx (Ojima et al. 1984; Scott et al. 1980). Importantly, the OB does not represent a passive relay station. Output cells receive inhibition from several classes of OB interneurons, such as periglomerular and granule cells, and are a target for centrifugal input and neuromodulation (Shipley and Ennis 1996). Given the OB is an intricate locus receiving primary input, transmitting output, and significant refinement of both input and output by interneurons, thus altering the information projected to $\mathrm{PCx}$ and other brain regions important for learning, the main OB constitutes an interesting place to study early sensory processing alterations as a result of aversive learning.

Early studies in adult rabbits were some of the first to report altered olfactory processing based on EEG data following aversive olfactory conditioning (Bressler 1988; Freeman and Schneider 1982). However, one of the drawbacks of EEG data is that it represents a mixed population of cell types, making it difficult to dissect changes in output cells from intrinsic OB cells. Similarly, early work in adolescent rats (P17) indicates learned olfactory aversion enhances 2-deoxyglucose (2-DG) uptake, an indicator of metabolic activity, in the glomerular layer (Coopersmith et al. 1986)); 
however, like EEG data, 2-DG assays have limited spatial resolution. This limitation has been rectified in recent years with calcium imaging of the genetically encoded calcium indicator, GCaMP, which allows for imaging of neural activity in genetically targeted OB cell types (Akerboom et al. 2012; Wachowiak et al. 2013). This methodological advance coupled with aversive olfactory conditioning permits investigation into several sources of potential change such as temporal and spatial coding as well as amplitude of evoked responses (Broussard et al. 2014; Ye et al. 2017).

Studies utilizing calcium imaging confirmed early reports of altered OB processing of conditioned odors following fear conditioning in several cell types. In anesthetized mice, enhanced responses of the conditioned stimulus have been reported in dendrites of both excitatory OB output cells (Fletcher 2012) and inhibitory periglomerular cells (Kass and McGann 2017), that persist as long as one month following single-day fear conditioning. Similar, but more robust associative learninginduced enhancements are seen in glomeruli of awake mice (Ross and Fletcher 2018), though whether these dendritic changes reflect altered coding at the soma of output cells remains a critical question. Importantly, under classical, single-day odor-shock conditioning paradigms, adult mice often acquire fear to the odor that was paired with foot shock but also extend that behavioral fear response to other odors never paired with shock, a process known as generalization.

Whether olfactory processing of odors to which fear is generalized also changes following fear conditioning has been of recent interest. Enhancement at the level of excitatory postsynaptic glomerular (Ross and Fletcher 2018) and inhibitory periglomerular (Kass and McGann 2017) responses has been reported for both the conditioned stimulus, and neutral, unconditioned odors. The enhanced responses may serve to increase the representational similarity between the conditioned and unconditioned odors, thus providing a neural basis for behavioral generalization (Ross and Fletcher 2018), though the exact mechanisms of both specific and global enhancements are still unknown. Potentiated glomerular responses in awake mice are not attributable to altered respiration or general fear states (Ross and Fletcher 2018) but reflect true plasticity of olfactory processing. Interestingly, associative learning is not required for conditioned stimulus glomerular enhancement, as responses are increased even when learning is blocked, but is required for the global enhancement of all odor processing, possibly indicating there are distinct mechanisms mediating these enhancements (Ross and Fletcher 2018).

In neonates $(<\mathrm{P} 10)$ odor-stroke conditioning causes learned odor preference and is associated with enhanced excitation of OB output cells (Yuan et al. 2003) and less fos activation in inhibitory granule cells (Woo et al. 1996). In addition, neonatal odor-stroke conditioning transiently increases cAMP (Cui et al. 2007) and phosphorylated CREB (pCREB) in mitral cells (McLean et al. 1999), which indicates increased transcription in OB output cells as a result of olfactory associative conditioning. Similar results have been found in young adolescent rats (P11) that undergo classical olfactory aversive conditioning and acquire learned fear. In young rats, olfactory aversive learning also increases pCREB, which is required for long-term, but not short-term, memory of learned 
aversion (Zhang et al. 2003). Therefore in early development, olfactory associative conditioning increases gene transcription directly in the $\mathrm{OB}$, which is likely important for long-term memory. Importantly, both mitral cells and granule cells exhibit markers of gene transcription in young rats following odor-shock pairing and pharmacologically augmenting transcriptional activation enhances learned aversion and recall (Wang et al. 2013). Together the results from neonatal appetitive conditioning and adolescent aversive conditioning convey associative learning initiates transcription in OB output cells and possibly inhibitory interneurons, leading to neural plasticity. Neonatal odor-stroke pairing is associated with CaMKII-mediated insertion of postsynaptic AMPA receptors (AMPAR) in the glomerular layer (Modarresi et al. 2016). Increased AMPARs appear to be required for both long-term memory of acquired odor-preference and behavioral specificity of preference to the conditioned odor (Cui et al. 2011; Modarresi et al. 2016). Data from neonatal and adolescent mammals suggests olfactory associative memory traces may form, at least initially, in the OB output neurons, a hypothesis common to insect olfactory learning (Faber et al. 1999; Menzel 2001; Yu et al. 2004). Increased AMPAR membrane insertion could provide this memory trace by increasing the postsynaptic glomerular response in adults following aversive conditioning. As AMPARs are an integral component of LTP and appear to mediate specificity of mammalian pup learning, they may be an attractive candidate for exploring the origin of specific versus global glomerular enhancements observed in adults. Postsynaptic glomerular responses can be enhanced directly by altering the excitability of mitral and tufted cells or indirectly by modifying inhibitory synaptic input onto OB output cells. There is significant evidence that odor-shock conditioning induces synaptic plasticity in both OB output cells and inhibitory interneurons (Tong et al. 2017; Wang et al. 2013), suggesting all cell types require further investigation to fully understand aversive-learning induced neuroplasticity in the OB.

It is important to note that pup learning appears to be highly specific to the CS in terms of both behavior and coding changes, while adult aversive learning is subject to generalization at both levels. This marks a central disparity between pups and adults that requires rigorous study. If the same mechanisms are responsible for CS-specific changes in both pups and adults, an additional mechanism is needed to explain non-specific coding and behavioral changes in adults. Certainly, additional molecular mechanisms or entirely different molecular mechanisms could underlie this divergence; however, neuromodulatory feedback is another interesting possibility. In young rats, glomerular disinhibition has been proposed as a mechanism for non-specific learned aversion (Okutani et al. 2003), which could be related to neuromodulatory feedback during acquisition. Again, much of the data collected from pups is prior to full brain development, where mature connections are still forming between $\mathrm{OB}$ and neuromodulatory regions.

The OB receives input from several neuromodulatory regions (Figure 1-1) which release norepinephrine (NE), acetylcholine (ACh), and serotonin (5-HT), all of which alter the balance of excitation and inhibition in the OB (for review see Fletcher and Chen 2010) and impact olfactory learning and plasticity. For example, NE is one of the widely studied neuromodulators in pup learning and is both necessary (Sullivan et al. 1994; 
Sullivan et al. 1989; Wilson et al. 1994) and sufficient (Harley et al. 2006; Shakhawat et al. 2012; Sullivan et al. 2000; Sullivan et al. 1989) to induce odor preference and associated plasticity in neonates. NE is also an important neuromodulator in adult olfactory associative learning (Brennan et al. 1998; Kroon and Carobrez 2009; RamirezGordillo et al. 2018; Zhang et al. 2010) though is not sufficient to produce behavioral outcomes in adults, likely due to developmental differences in norepinephrine (Moriceau and Sullivan 2004; Pandipati and Schoppa 2012). Similarly, the related modulator, octopamine, is required for insect aversive learning (Iliadi et al. 2017). NE fibers project from the locus coeruleus to the mammalian $\mathrm{OB}$ where they terminate most densely in layers containing dendrites of mitral cells and inhibitory granule cells, which express NE receptors (Levy et al. 1999; Shipley and Ennis 1996). This allows NE to modulate the activity of both cell types and regulate learning-induced plasticity in OB circuits (Yuan et al. 2003). Interestingly in adult rabbits odor-shock training, infusion of NE antagonists into the OB suppresses learning-induced OB activation pattern changes (Gray et al. 1986), further indicating the importance of NE in adult aversive-learning induced plasticity.

ACh is also required for pup and adult mammalian olfactory associative learning (Chan et al. 2017; Chaudhury et al. 2009; Devore et al. 2012; Hellier et al. 2012; Kroon and Carobrez 2009), and may regulate olfactory fear generalization in adults (Pavesi et al. 2012). However, studies addressing the role of ACh in olfactory fear learning mostly use systemic administration of $\mathrm{ACh}$ antagonists, making it difficult to discern whether $\mathrm{ACh}$ is specifically required in the $\mathrm{OB}$ for aversive-learning induced plasticity. ACh fibers from the basal forebrain, most specifically the horizontal limb of the diagonal band of Broca (Levy et al. 1999), terminate primarily in the glomerular layer, where they synapse onto periglomerular cells, and in the internal plexiform layers, where they synapse on granule cell dendrites (Shipley and Ennis 1996), but there is also evidence of non-synaptic interactions between ACh axons and mitral cells (Kasa et al. 1995). The abundance of synaptic and non-synaptic interactions between $\mathrm{ACh}$ axons and $\mathrm{OB}$ cell types provides a complex framework for ACh mediated learning-induced synaptic plasticity that requires investigation.

In addition, little is known about 5-HT modulation of olfactory associative learning-induced OB plasticity, even though 5-HT is required for pup (McLean et al. 1996; McLean et al. 1993), adult (Marchetti et al. 2000), and insect learning (Johnson et al. 2011). Interestingly, in the short-nosed fruit bat, depletion of 5-HT prevents olfactory associative learning as well as associated OB plasticity, such as upreglation and phosphorylation of CREB (Ganesh et al. 2010), which was necessary for olfactory aversive learning in young rats (Zhang et al. 2003). The OB receives 5-HT input from raphe nuclei (Levy et al. 1999) to all layers of the bulb; however, the densest projections occur in the glomerular layer (McLean and Shipley 1987; Shipley and Ennis 1996). The OB output cells and granule cells to a lesser extent (Hamada et al. 1998; McLean et al. 1995), express 5-HT receptors, indicating a role for direct and indirect 5-HT modulation of OB output cells. Given the substantial neuromodulatory input to the OB and the requisite nature of these transmitters in olfactory associative learning, more thorough 
exploration of aversive learning-induced changes in neuromodulatory feedback to the $\mathrm{OB}$ and the role it plays in OB plasticity is required.

Together these studies demonstrate the adult $\mathrm{OB}$ undergoes dramatic changes following aversive olfactory learning in numerous cell types. While work regarding OB plasticity following pup aversive learning is sparse, work in adolescent aversive conditioning and pup appetitive conditioning demonstrate similar molecular changes that ultimately lead to transcription and insertion of AMPARs in the glomerular layer following associative learning. The conservation of learning-induced AMPAR insertion across developmental stages and despite differences in training paradigm offers a parsimonious mechanism for sensory plasticity (Sullivan and Wilson 2003). Importantly, this constitutes an example of identified molecular plasticity in pup learning with functional physiological and behavioral consequences that mirrors the observed aversive learning-induced changes in adults. The putative mechanism of pup plasticity could also provide the neural basis for increased sensitivity and enhanced glomerular responses following adult aversive learning. Furthermore, these local OB synaptic changes may be part of larger circuit-level changes in neuromodulatory input. Future work should attempt to identify these types of parallels between pups and adults and apply results obtained from pup learning to investigations of adult olfactory aversive conditioninginduced plasticity.

\section{Piriform Cortex}

The primary olfactory cortex is the $\mathrm{PCx}$, which can be subdivided along the anterior-posterior axis based on the inputs they receive (Figure 1-1). In terms of OB output cells, the anterior $\mathrm{PCx}(\mathrm{aPCx})$ receives dense input from both mitral and tufted cells, whereas the posterior PCx (pPCx) receives sparser innervation, primarily from mitral cells (Igarashi et al. 2012; Nagayama et al. 2010). OB input to PCx does not exhibit conserved spatial patterning, as observed at the $\mathrm{OB}$ glomerular layer, providing a flexible template for learning-induced changes (Ghosh et al. 2011; Miyamichi et al. 2011; Sosulski et al. 2011; Stettler and Axel 2009). The PCx as a whole receives projections from a variety of neuromodulatory centers, many of which are thought to play a critical role in learning and memory, making the PCx an interesting location for studying aversive learning-induced coding changes. In addition, $\mathrm{pPCx}$ receives heavy associative input from aPCx (Illig and Wilson 2009) and projections from the amygdaloid complex (Majak et al. 2004), which may indicate increased involvement in associative fear learning. There is also evidence to support that in addition to differences in input, the aPCx and pPCx play different roles in regard to olfactory learning, though the exact contributions of each has yet to be clearly defined (Chabaud et al. 2000; Litaudon et al. 1997; Mouly et al. 2001).

Several studies in rodents demonstrate PCx alterations following adult olfactory fear learning. For example, single-day odor-shock pairing in adult rats leads to transient potentiation of CS-induced field potentials in the PCx 24 hours later that coincides with learned freezing (Sevelinges et al. 2004). These changes were specific to the pPCx, while 
no learning-induced changes were evident in aPCx. Interestingly, while adult rats also extend acquired freezing beyond the CS, whether neutral, untrained odorants also evoked altered pPCX field potentials was not tested. In a similar, single-day aversive conditioning task, BDNF mRNA was upregulated in aPCx 2 hours after conditioning in all odor exposed groups but only upregulated in $\mathrm{pPCx}$ in groups subjected to odor-shock pairing (Jones et al. 2007), suggesting aPCx may code information regarding basic odor experience while $\mathrm{pPCx}$ codes olfactory learning in adults. The odor-shock pairinginduced upregulation of BDNF in pPCx was concomitant with upregulated amygdaloid BDNF, further suggesting an interaction between amygdaloid activation by aversive learning and $\mathrm{pPCx}$ alterations in adults.

When pups $(<\mathrm{P} 10)$ are subjected to aversive conditioning paradigms they demonstrate paradoxical preference. Interestingly under such circumstances, pups exhibit significant learning-induced changes in the PCx, but those changes are mostly specific to aPCx. For example, odor-shock pairing that results in paradoxical preference is associated with increased aPCx c-fos activation (Roth and Sullivan 2005) and increased 2-DG uptake, a measure of cellular metabolic activity (Raineki et al. 2009). Remarkably if pups undergo extreme odor-shock training or undergo training at a later age $(>\mathrm{P} 10)$, they acquire aversion and enhancement of 2-DG uptake in $\mathrm{pPCx}$ rather than $\mathrm{aPCx}$ (Raineki et al. 2009). This suggested the PCx may place hedonic value on olfactory learning, where the aPCx is associated with olfactory preference and $\mathrm{pPCx}$ with odor aversion; however, this claim has not yet been thoroughly explored and such stringent definitions preclude understanding how cortical processing as a whole changes following adult olfactory aversive learning.

A more recent study subjected adult rats to either single-day classical or discriminant odor-shock conditioning and recorded single-unit activity in aPCx to the CS as well as similar odors (Chen et al. 2011). This allowed for direct comparisons of altered aPCx coding when fear learning was generalized (as is the case in classical conditioning) or specific (discriminant conditioning). Despite previous data indicating lack of aPCx cfos activity or 2-DG uptake changes following adult aversive conditioning, both classical and discriminant learning altered the single-unit activity in aPCx. Interestingly, classical conditioning broadened the odor-evoked receptive field size of aPCx units while discriminant conditioning narrowed receptive fields. These findings correlate with both behavioral generalization and studies involving OB physiology (Kass and McGann 2017; Ross and Fletcher 2018).

It has been hypothesized that OB coding changes following associative learning signify odor salience but require PCx alterations to code hedonic value (Jones et al. 2008; Raineki et al. 2009) and mediate learned behaviors (Choi et al. 2011). Despite the significance placed on the PCx in regard to associative learning, relatively few studies on the mechanisms by which PCx coding is altered following adult aversive learning have been conducted. Furthermore, it remains to be seen whether observed PCx plasticity is intrinsic to $\mathrm{PCx}$ or is due to altered input from the $\mathrm{OB}$, higher processing centers, or neuromodulatory regions. During adult odor-shock conditioning, a study using high temporal resolution microdialysis ascertained transient increases in both GABA and 
glutamate in PCx following each odor-shock pair that was not present during odor only exposures (Hegoburu et al. 2009). The transient increase in PCx glutamate was preceded by glutamate activation in the amygdala, possibly indicating the amygdala is responsible for increased glutamate in the PCx during odor-shock trials. Amygdalar modulation of PCx is made possible by a direct pathway between the two structures (Majak et al. 2004) and functionally, optogenetic stimulation of amygdalar neurons alters odor-evoked ensemble activity in the PCx (Sadrian and Wilson 2015). Plasticity, by means of strengthening synaptic connections between PCx and the amygdala could explain such a phenomenon; however, this causal relationship has not yet been directly tested in the context of adult aversive learning.

The PCx, like the $\mathrm{OB}$, receives considerable neuromodulatory input from noradrenergic, cholinergic, and serotonergic centers (Shipley and Ennis 1996). While systemic injections of NE, ACh, and 5-HT antagonists impair olfactory associative learning (Kroon and Carobrez 2009; Marchetti et al. 2000; McLean et al. 1996), almost nothing is known about the role of these neurotransmitters in the PCx in the context of olfactory aversive learning. The effect of $\mathrm{PCx} 5$-HT during associative learning is completely unexplored; however, optogenetic stimulation of 5-HT inputs decreases spontaneous PCx firing without affecting odor-evoked PCx activity (Lottem et al. 2016), which could facilitate cortical olfactory learning and plasticity. Blockade of NE receptors in PCx prevents acquisition of olfactory associative appetitive learning in both pups (Ghosh et al. 2015; Morrison et al. 2013) and adults (Shakhawat et al. 2015) and NE stimulation reverses learning-induced hyperexcitability of PCx pyramidal cells (Brosh et al. 2006), indicating a function for NE in olfactory learning-induced PCx plasticity. Data from electrophysiological and computational studies indicate ACh places $\mathrm{PCx}$ in a permissive state for both learning and recall in adult associative learning (Barkai et al. 1994; Barkai and Hasselmo 1997; Hasselmo et al. 1992) by simultaneously suppressing synaptic transmission and enhancing synaptic modification (Linster and Hasselmo 2001) and enhancing long-term potentiation in PCx pyramidal cells (Hasselmo and Barkai 1995; Patil et al. 1998). This mechanism allows for pattern separation and discriminability following appetitive learning, as evidenced by the fact that pharmacological inhibition of ACh in PCx leads to behavioral generalization (Wilson 2001) while systemic ACh agonists enhance behavioral discrimination (Doty et al. 1999). Together, these results provide strong evidence that these neuromodulators are required in the $\mathrm{PCx}$ for associative learning and that they may mediate synaptic plasticity that leads to behavioral outputs of learning; however, this work must be repeated in the context of aversive learning to ascertain how an aversive learning paradigm affects neuromodulation to produce neuroplasticity that results in generalized fear.

\section{Conclusion}

Olfactory associative conditioning induces neural plasticity to enable behavioral responses to a learning experience. Current evidence supports plasticity throughout the mammalian olfactory system, at the initial stages of processing in OSNs and OB output cells as well in the primary olfactory cortex. In cases of olfactory aversive conditioning, 
pups exhibit paradoxical preference specific to the CS, whereas adults express generalized aversion to the CS and non-conditioned odorants, which has hindered drawing parallels between pup and adult mechanisms of aversive learning. Importantly, there are developmental differences between pups and adults which affect this behavioral dichotomy, including the lack of amygdala plasticity in neonates (for review see Landers and Sullivan 2012). While some differences occur in behavioral responses following aversive conditioning between pups and adults, there is significant conservation in underlying mechanisms of neural plasticity.

Despite these differences, associative learning in pups and aversive learning in adults transforms olfactory processing, especially at the OB glomerular layer. Glomerular layer changes occur either at the presynaptic OSN component or at the postsynaptic component. Importantly, while OSN changes have been reported following pup and adult aversive conditioning, the conditioning paradigms took place over several days making it difficult to ascertain whether the proposed mechanisms account for changes occurring within 24 hours of training. How glomerular processing as a whole changes following aversive learning has yet to be delineated; however, associative learning in both pups and adults increases neural activity of $\mathrm{OB}$ output cells, either directly or indirectly through altering inhibitory drive onto output cells.

The proposed mechanisms of learning-induced AMPAR insertion in pups, leading to enhanced excitation of OB output cells echoes enhanced odor-evoked glomerular responses in adults following odor-shock learning. In other systems, AMPAR membrane insertion serves to potentiate the synapse, typically via the postsynaptic neurons (Luscher and Malenka 2012; Park et al. 2006; Wang et al. 2008), which could explain enhancement of CS-specific OB output cells (Ross and Fletcher 2018). Regardless of developmental stage, associative conditioning potentiates neural activity in $\mathrm{PCx}$ as well as the OB. In cases of learned aversion, both pups and adults exhibit enhanced activity in pPCx. This likely reflects a combination of input from the OB, which itself is increased following aversive learning, as well as from aPCx and other regions, possibly including the amygdala. Similar mechanisms may also underlie adult olfactory appetitive conditioning (Lebel et al. 2001; Quinlan et al. 2004; Reuveni et al. 2013; Saar et al. 2012; Tong et al. 2014) and could offer insights and testable hypotheses to advance adult olfactory aversive learning; however, adult appetitive conditioning typically utilizes multiple training sessions over several days. Such studies represent gradual, cumulative learning that results in more specific behavioral learning rather than rapid, single-day learning with broad behavioral generalization, and could, therefore, use different or additional mechanisms.

It is important to remember that learning-induced plasticity in both the $\mathrm{OB}$ and $\mathrm{PCx}$ require neuromodulatory and centrifugal feedback during acquisition. These olfactory centers both receive dense innervation from noradrenergic, cholinergic, and serotonergic regions. While the exact roles of each have yet to be identified for aversive learning-induced $\mathrm{OB}$ and $\mathrm{PCx}$ plasticity, there is increasing evidence that they facilitate learning. For example, NE, ACh, and 5-HT have all been shown to promote synaptic plasticity by initiating intracellular signaling cascades (Jerusalinsky et al. 1997), 
increasing translation (Maity et al. 2015), or trafficking receptors for membrane insertion (Hu et al. 2007; Mlinar et al. 2015). In addition, each of these neuromodulators alters the balance of excitation and inhibition in the olfactory pathway (Fletcher and Chen 2010), which could provide a basis for generalized versus specific aversive learning (Okutani et al. 2003). Therefore, a picture emerges where neuromodulators are inextricable from olfactory plasticity. Both neurotransmitter systems and the capacity for plasticity differ between pups and adults (Bower 1990; Herlenius and Lagercrantz 2004; Le Jeune and Jourdan 1991; McLean and Shipley 1991; Rea and Nurnberger 1986; Shionoya et al. 2006; Wilson and Leon 1988), which may limit complete parallels between mechanisms of learning-induced olfactory plasticity. In addition to investigating whether identified mechanisms of learning-induced plasticity in pups applies to aversive learning-induced plasticity throughout the olfactory system in adults, future studies should aim to identify the role of neuromodulators in olfactory plasticity. 


\section{CHAPTER 2. LEARNING-DEPENDENT AND -INDEPENDENT ENHANCEMENT OF MITRAL/TUFTED CELL GLOMERULAR ODOR RESPONSES FOLLOWING OLFACTORY FEAR CONDITIONING IN AWAKE MICE $^{2}$}

\section{Introduction}

Associative fear learning, in which an organism learns that a given conditioned stimulus (CS) predicts an aversive outcome, produces behavioral fear upon subsequent encounters with that stimulus. In addition, this form of learning often generates robust generalization, the expansion of fear from the threat-predictive CS to other, unlearned stimuli (Pavlov, 1927; Pavesi et al., 2012; Resnik and Paz, 2015). Studies in different sensory systems demonstrate that fear learning alters the neural networks responsible for encoding the CS-fear association (Bakin and Weinberger, 1990; Rogan et al., 1997; Maren, 2003a, b, 2005; Walker et al., 2005; Weinberger, 2007; Herry et al., 2008; Johansen et al., 2011; Letzkus et al., 2011; Sadrian and Wilson, 2015), but it remains unclear how representations of non-conditioned stimuli are altered, especially in relation to behavioral generalization.

The mouse olfactory system offers an excellent model in which to study such effects on sensory representations. Olfactory bulb $(\mathrm{OB})$ glomeruli are the sites of synaptic contact between olfactory sensory neuron (OSN) axons and the dendrites of the $\mathrm{OB}$ output cells, mitral/tufted (M/T) cells (Buck and Axel, 1991). Odor-induced OSN activation generates unique spatiotemporal patterns of glomerular activity that form the initial basis of odor identity (Wachowiak and Cohen, 2001; Spors and Grinvald, 2002; Bozza et al., 2004; Mori et al., 2006; Fletcher et al., 2009; Storace and Cohen, 2017). OB imaging studies using optical indicators of neuronal activity expressed in OSNs (Kass et al., 2013), M/T cells (Fletcher, 2012), and inhibitory periglomerular cells (Kass and McGann, 2017) report increased responses to the trained odor following olfactory fear conditioning. While these studies clearly indicate learning alters glomerular representations of odorants, they only investigated effects in anesthetized mice. Recent discoveries demonstrate distinct differences in odor responsivity between awake and anesthetized states in both excitatory output cells and various inhibitory interneurons (Kato et al., 2012; Blauvelt et al., 2013; Wachowiak et al., 2013).

Furthermore, other than a single recent study of inhibitory interneuron responses in anesthetized mice (Kass and McGann, 2017), little is known regarding how learning to fear the CS might affect sensory processing of neutral, unlearned odorants, especially in awake animals. Importantly in the anesthetized state, $\mathrm{M} / \mathrm{T}$ cells are more broadly tuned to odor input, leading to less efficient odor identity coding, and both inhibitory interneuron

\footnotetext{
${ }^{2}$ Reprinted from final submission with permission of The Society for Neuroscience. Ross, J.M. and Fletcher, M.L. (2018) Learning-dependent and -independent enhancement of mitral/tufted cell glomerular odor responses following olfactory fear conditioning in awake mice $J$ Neurosci 38:4623-4640 doi:10.1523/JNEUROSCI.3559-17.2018; permission conveyed through Copyright Clearance Center, Inc.
} 
activity and centrifugal feedback are reduced in anesthetized states; each of these factors likely play an important role in learning-induced glomerular odor coding alterations.

Therefore, the extent to which olfactory aversive learning modulates sensory processing of the CS as well as neutral odorants in awake mice is an important question in relation to behavioral fear generalization.

Using awake, behaving calcium imaging we report, for the first time, that a single day fear conditioning paradigm leads to long-lasting increased glomerular responses to not just the learned odor (CS) but also to other, non-conditioned odors. Such enhancements lead to increasingly overlapping glomerular representations between the conditioned and neutral odors. Furthermore, we demonstrate this global glomerular enhancement is dependent on amygdalar activation during acquisition, suggesting global enhancements require associative fear learning. Additionally, the enhancements could not be blocked by inhibiting the amygdala during expression, meaning the enhancements occur during or shortly after learning acquisition. Together, these results indicate that classical olfactory fear learning induces changes as early as the first synapse in the olfactory system and alters glomerular odor coding in a global manner that may contribute to behavioral generalization.

\section{Methods and Materials}

\section{Animals}

All imaging and behavioral experiments used adult (8-14 weeks) male and female mice generated from crossing FVB/N-Tg(Thyl-cre) 1 Vln/J with B6;129SGt(ROSA)26Sortm38(CAG-GCaMP3)Hze/J, such that the resulting mice expressed the fluorescent calcium indicator GCaMP3 under the Thy1-promotor. Resultant mice express GCaMP3 in olfactory bulb excitatory neurons, such as M/T cells. The Thy1 promoter also drives expression in cortical fibers (Chen et al., 2012) and, therefore, a portion of GCaMP signal measured in the OB could arise from odor-evoked cortical fiber activation. However, previous work indicates axons from piriform cortex terminate sparsely in the glomerular and external plexiform layers and most densely in the OB granule cell layer, the deepest OB layer (Otazu et al., 2015). Fluorescence from such a deep or sparse signal likely contributes little to the signal measured at the glomerular surface.

Male and female mice were equally distributed between groups (i.e. 3/2 in groups of 5, 2/2 in groups of 4, etc). Importantly, we did not notice any differences in behavioral fear expression or physiology between male and female mice. All experimental protocols were approved by the University of Tennessee Institutional Animal Care and Use Committee. 


\section{General Methodology}

\section{Surgical procedures}

Mice were anesthetized with ketamine/xylazine $(100 / 10 \mathrm{mg} / \mathrm{kg}$, i.p.) and given analgesic injections (carprofen $10 \mathrm{mg} / \mathrm{kg}$ s.c.) prior to surgery. Mice were secured in a custom stereotaxic apparatus (Narishige) and the bone overlying the dorsal surface of the olfactory bulb $(\mathrm{OB})$ was thinned to create a cranial window for optical imaging. Additionally, some mice were implanted with bilateral cannula in the basolateral amygdala (BLA; Bregma: -1.5 AP, $\pm 3.3 \mathrm{ML},-5.0 \mathrm{DV})$. An anchor screw was inserted into the frontal or parietal bone and the entire skull was sealed with a thin layer of superglue (BSI). A custom made stainless steel headbar was attached to the posterior surface of each mouse's skull for head fixation during imaging experiments. The entire skull was then covered with acrylic dental cement and mice were allowed two days to fully recover before experimentation.

\section{Drug infusions and placement verification}

Mice with BLA cannula received bilateral intra-cannula muscimol (MUSC; $0.5 \mu 1$ of $0.5 \mu \mathrm{g} / \mu \mathrm{l}$ delivered at a rate of $0.25 \mu \mathrm{l} / \mathrm{min}$ ) or an equal volume of vehicle (VEH; Ringers) infusions via a microsyringe pump. Injectors were left in place for an additional 2 minutes for diffusion. After completion of all experiments, mice with BLA cannula were perfused and brains were removed and sectioned to verify cannula placement within the BLA. Some brains were infused with Chicago Sky Blue dye (Sigma) prior to perfusion to assess possible MUSC spread. See Detailed Methodology regarding timing of drug infusions.

\section{Odorants}

An odorant panel consisting of 5 different odorants (ethyl valerate (E5), ethyl butyrate (E4), ethyl hexanoate (E6), benzaldehyde (BZ), 2-heptanone (2H)) was used (Sigma). E5, an ethyl ester with a 5 carbon chain, was selected as the conditioned stimulus for fear conditioning following previous work in our lab (Pavesi et al., 2012). The other odorants were selected for their perceptual and representational similarity or dissimilarity to E5 to assess responses to chemically diverse odors. E4 and E6 were selected as structurally similar odorants, as they are also classified as ethyl esters with a 4- and 6- carbon chain, respectively. BZ, an aldehyde, and 2H, a ketone, were selected as structurally dissimilar odorants. Importantly, all odors were known to activate glomeruli on the dorsal surface of the olfactory bulb.

\section{Optical imaging}

During imaging experiments, mice were head-fixed to a custom-built treadmill (Chettih et al., 2011; Heiney et al., 2014). The treadmill allowed mice to dictate their forward/reverse motions while remaining head-fixed. All mice were awake for imaging with the exception of mice in Experiment 3 (below). Imaging was performed using a 
Scientifica Slicescope equipped with a 4x objective (Olympus). All physiological imaging data was derived from changes in fluorescence measured from the dorsal $\mathrm{OB}$ surface, which reflects activity in the glomerular layer, likely from dendrites of $\mathrm{M} / \mathrm{T}$ cells. The dorsal surface was illuminated with a LED light source centered at $480 \mathrm{~nm}$. GCaMP3 signals were band-pass filtered with a Chroma emission filter (HQ535/50) and collected using a CCD camera at $25 \mathrm{~Hz}$ (NeuroCCD-SM256, Redshirt Imaging). During imaging experiments, all mice received at least 4 trials of each odor used in each experiment (detailed below) and at least 4 no odor trials presented in a random order. Imaging trials lasted 5 seconds and consisted of $1 \mathrm{~s}$ of no odor, followed by a $2 \mathrm{~s}$ odor presentation, and $2 \mathrm{~s}$ no odor $(\mathrm{ITI}=1-2 \mathrm{~m})$. Control no odor trials also lasted 5 seconds. All odors were delivered to the nose via a flow-dilution olfactometer. Separate flow controllers for the clean air and the pure odorant vapor mixed the flow streams at the end of the odor delivery system to achieve an approximate concentration of $0.5 \%$ saturated vapor (s.v.) at a flow rate of $0.7 \mathrm{~L} / \mathrm{min}$.

\section{Behavior}

\section{Olfactory fear conditioning}

All olfactory fear conditioning occurred in a standard shock chamber (Coulbourn Instruments). Mice that underwent odor-shock conditioning (Paired) received 6 E5-foot shock pairings ( $10 \mathrm{~s}$ E5 co-terminating with a $0.8 \mathrm{~mA}, 0.5 \mathrm{~s}$ foot shock). Mice in the Shock only condition received 6 unpaired foot shocks of the same intensity, while mice in the Odor only condition received 6 E5 presentations of the same duration. Approximately 24 hours after conditioning, mice were placed in a novel, custom-made testing chamber. The chamber is composed of two halves separated by a perforated wall. Mice were placed in one half of the chamber while odor delivery lines were fed through the other side, allowing odors to permeate both halves of the chamber without directly blowing on mice. The testing chamber was enclosed in an isolation chamber (Coulbourn Instruments) designed to isolate mice from all external sounds controlling odor delivery as well as any visible light. An infrared light was placed inside the testing chamber and all tests were recorded with a compatible camera. Mice were allowed a 5-10 minute acclimation period in the chamber before initiation of the testing protocol, in which we assessed behavioral freezing, a widely used measure of fear, for each of the 5 odors used during imaging experiments. This allowed testing of specific fear to the conditioned stimulus (E5) as well as generalized fear towards neutral odors (E4, E6, BZ, and $2 \mathrm{H})$ never paired with shock. Testing consisted of one 20 s presentation of each odor (ITI $=3 \mathrm{~m})$, starting in the second minute of the test session. The first $60 \mathrm{~s}$ of each testing session were absent of odors and were used as a baseline measure of freezing. All testing odors were intensity matched and diluted in mineral oil to achieve an approximate headspace concentration of $200 \mathrm{ppm}$. Odors were delivered to the testing chamber through dedicated lines. To ensure olfactory fear conditioned mice freeze to odor cues rather than air pressure changes or auditory cues in the testing chamber, we conditioned a small cohort of mice $(n=3)$ to E5 and tested their freezing to E5 as well as clean air delivered under the same parameters. Mice do not freeze to clean air more than baseline freezing $\left(\mathrm{t}_{7}=1.472, \mathrm{p}=0.185, \mathrm{r}^{2}=0.236\right)$ 
but freeze significantly more to E5 than either clean air $\left(\mathrm{t}_{10}=11.585, \mathrm{p}<0.0001, \mathrm{r}^{2}=\right.$ $0.931)$ or baseline freezing $\left(t_{7}=16.43, p<0.0001, r^{2}=0.975\right)$.

\section{Auditory fear conditioning}

During fear conditioning, Tone-shock mice $(\mathrm{n}=3)$ were placed in the same shock chamber as olfactory fear conditioned mice but received 6 tone-shock pairings $(10 \mathrm{~s}$, $10 \mathrm{kHz}, 82 \mathrm{~dB}$, co-terminating with a $0.8 \mathrm{~mA}, 0.5 \mathrm{~s}$ foot shock). 24 hours later they were placed in the same novel context as olfactory conditioned mice but experienced 4-20s presentations of the paired tone to confirm tone-shock learning.

\section{Detailed Methodology}

For convenience, each figure of a new experiment includes a schematic detailing the odorants used during imaging sessions, time course of imaging and behavioral experiments, and the training and testing paradigms used.

\section{Experiment 1}

Mice underwent three consecutive days of chronic awake imaging (Pre1, Pre2, and Post) to assess glomerular odor representations for each of the five odors in the odorant panel. Following the Pre2 imaging session, mice were split into three groups for fear conditioning: Odor only, Shock only, and Paired, and then subjected to testing as detailed above $(\mathrm{n}=5$ each). Mice experienced each of the 5 odors in the odorant panel during imaging and testing but only experienced E5 during training. Mice were awake for all aspects of Experiment 1.

\section{Experiment 2}

Mice $(n=4)$ underwent two consecutive days of chronic awake imaging (Pre1 and Pre2) before fear conditioning to E5. Approximately 72 hours after paired conditioning, mice underwent testing and a final imaging session (Post3). Mice were awake for all aspects of Experiment 2 and experienced each of the 5 odors in the odorant panel during imaging and testing. During training, mice only experienced E5.

\section{Experiment 3}

Mice $(n=3)$ underwent the exact same experimental protocol as those in Experiment 1 except that all imaging sessions (Pre1, Pre2, and Post) were completed under anesthesia (100/10mg/kg i.p. ketamine/xylazine). Mice were awake for all behavioral aspects of Experiment 3 (training and testing) but were anesthetized for all imaging sessions. Mice experienced each of the 5 odors in the odorant panel during imaging and testing but only experienced E5 during training. 


\section{Experiment 4}

Mice $(n=3)$ underwent three consecutive days of chronic awake optical imaging, but only experienced an abbreviated panel of odorants consisting of E5 and BZ during imaging sessions. The abbreviated panel was used due to the Pre-Post design of the Post imaging session (Post1 and Post2). Using all 5 odors would dramatically increase the length of the Post imaging session, while using an abbreviated panel of odors kept the Post imaging sessions at approximately the same length as all previous Post imaging sessions. Following the Pre2 imaging session, mice were subjected to auditory fear conditioning and testing. The final, post-conditioning, imaging session was split into two halves (Post1 and Post2). During Post1, we assessed baseline post-training glomerular odor representations of E5 and BZ. During Post2, each odor trial was preceded by a 10s presentation of the same tone mice experienced during auditory fear conditioning to assess whether global fear states impact glomerular responses. Mice were awake for all aspects of Experiment 4. During training, mice only experienced the $10 \mathrm{kHz}, 82 \mathrm{~dB}$ conditioning tone.

\section{Experiment 5}

During surgery, these mice received bilateral BLA cannula. Mice underwent the experimental procedures outlined in Experiment 1 but received infusions of either VEH or MUSC ( $\mathrm{n}=5$ each) 10 minutes before odor-shock conditioning to transiently inactivate the BLA during acquisition. Mice were awake for all aspects of Experiment 5. During each of the three awake imaging sessions and during testing, mice experienced all five odors in the odorant panel. During training, mice only experienced E5.

\section{Experiment 6}

During surgery, these mice received bilateral BLA cannula. Mice underwent three consecutive days of awake imaging, but only experienced an abbreviated odorant panel of E5, E4, and BZ during imaging. The abbreviated panel was again used due to the PrePost design of the final imaging session, which was split into two halves (Post1 and Post2), similar to Experiment 4. This time we used 3 odors in our abbreviated panel in order to assess changes to the CS (E5) as well as one structurally similar (E4) and one structurally dissimilar (BZ) odorant. In this experiment, all mice were fear conditioned to E5 and tested for fear to each of the five odors before the final imaging session. This allowed us to measure generalization to novel odorants (E6 and $2 \mathrm{H})$. Between Post1 and Post2, mice were left head-fixed on the treadmill and received infusions of either VEH or MUSC $(\mathrm{n}=5$ each) to transiently inactivate the BLA during expression. Ten minutes after the infusion, we resumed the Post2 imaging session half.

\section{Quantification and Statistical Analyses}

Physiological data collected is based on number of responsive glomeruli during the first peak respiration (a 5 frame average) following odor onset and analysis of this 
data was achieved by collapsing data into a single value for each glomerulus representing its mean daily response. For visual clarity, graphical representation of this same data was reduced to a single value representing the mean daily glomerular response for each odor (i.e., five data points per mouse per day) and presented as mean \pm SEM (standard error of the mean). Statistics were analyzed using R statistical analysis software and IBM SPSS 22.0. Equal variances were tested for all comparison data by Levene's test or Mauchly's test of Sphericity, and suitable corrections were made when necessary. Parametric statistical tests including independent samples t-tests, ANOVAs and Repeated Measures ANOVAs, and post hoc analyses were conducted when main effects were found to be significant (Dunnett's T for ANOVAs and Bonferroni with adjustment for multiple comparisons for RM ANOVAs). ANOVAs were performed for all behavioral freezing and MIF data, unless otherwise noted. RM ANOVAs were performed for analyses of glomerular response data over days. T-tests were performed to compare $\%$ change data. $\mathrm{N}$ values for behavioral statistics represent number of individual mice while $\mathrm{n}$ values for glomerular statistics represent the number of averaged glomerular responses.

As the glomerular fluorescent signal reflects respiratory rhythm at slower respiratory rates (Spors et al., 2006; Fletcher et al., 2009; Wachowiak et al., 2013), respiratory rate analyses were conducted using the raw fluorescent traces of each imaging trial. This method has reliably identified respiratory events when compared to respiratory signal taken from a piezoelectric device wrapped around the animal's chest and by measuring intranasal pressure. Raw fluorescent traces were picked from a random glomerulus on the dorsal surface of the OB exhibiting distinct respiratory signal. Raw fluorescent traces were first smoothed by applying a rolling average of 3 frames across the entire trace. An algorithm was then used to detect each respiratory peak and to calculate the mean instantaneous frequency (MIF). Using this method, we find respiration frequencies across a broad range $(\sim 2-6 \mathrm{~Hz})$, which is in line with reported sniffing rates of head-fixed mice as measured intranasally (Shusterman et al., 2011; Blauvelt et al., 2013; McAfee et al., 2016). Independently, we have also recorded awake, head-fixed sniff rates in this range during odor presentations using intranasal thermistor probes. For comparisons, odor-evoked MIF was restricted to the first four respirations after odor onset.

Spatial maps of stimulus-evoked glomerular activity were generated as previously described (Fletcher et al., 2009; Ogg et al., 2015). First, each trial was corrected for photobleaching. Then the odor-evoked change in fluorescence $(\Delta \mathrm{F})$ was then calculated by subtracting the average fluorescence of five frames centered around the peak of the respiration immediately preceding odor onset from five frames centered around the odorevoked respiratory peak. Relative fluorescence change $(\Delta \mathrm{F} / \mathrm{F})$ was then calculated by dividing the odor-evoked change in fluorescence $(\Delta F)$ by the average resting fluorescence gathered in the first 5 frames of the imaging trial. For spatial correlation analyses, the final odor map for each odor was obtained by averaging all same-odor trials within each day to generate a daily mean odor map. Using ImageJ, daily mean odor maps were aligned across odors and days for individual animals. Pseudocolored maps for individual animals are shown here as representing $\Delta \mathrm{F} / \mathrm{F}$ values. Next, a spatial correlation was performed using the corr2 function in MATLAB to compare the daily mean odor map of 
each neutral odor to the map of E5 before (Pre2) and after (Post) training for the Paired, Odor only, and Shock only groups. The corr2 function produces a single Pearson's 2-D correlation coefficient for the entirety of the representative maps, including both responsive and non-responsive regions, which allows for a holistic similarity comparison.

For quantitative analysis of individual glomerular responses, trials were first separated by odor presentation. There were a minimum of 4 trials for each odor on each day. Glomeruli were considered responsive if they met previously defined criteria: if the mean $\Delta \mathrm{F} / \mathrm{F}$ value on Pre1 was greater than the mean $+2 \mathrm{SD} \Delta \mathrm{F} / \mathrm{F}$ value obtained from regions containing no odor-evoked activity (Fletcher, 2011; Ogg et al., 2015). The individual glomerular responses for each trial were calculated from the $\Delta \mathrm{F} / \mathrm{F}$ measured at the center of each defined glomerulus ( $2 \times 2$ pixel average). Since glomerular responses spanned a wide range from animal to animal, responses were normalized to allow for pooling across subjects. Every individual responsive glomerulus was then normalized within each odor to its maximum observed $\Delta F / F$ response on Pre1 to that odor, such that the largest observed Pre1 response was equal to 1. In Experiment 1, maximum observed raw responses for each odor did not differ statistically across groups on Pre 1 (E5: F2, $12=$ $0.240, p=0.790 ; E 4: F_{2,12}=0.023, p=0.975 ; E 6: F_{2,12}=0.268, p=0.770 ; B Z: F_{2,12}=$ $\left.0.395, \mathrm{p}=0.682 ; 2 \mathrm{H}: \mathrm{F}_{2,12}=0.161, \mathrm{p}=0.853\right)$ or Pre2 $(\mathrm{E} 5: \mathrm{F} 2,12=0.495, \mathrm{p}=0.621$; E4: $\mathrm{F}_{2,12}=0.160, \mathrm{p}=0.854 ; \mathrm{E} 6: \mathrm{F}_{2,12}=0.425, \mathrm{p}=0.664 ; \mathrm{BZ}: \mathrm{F}_{2,12}=0.277, \mathrm{p}=0.763 ; 2 \mathrm{H}$ : $\mathrm{F}_{2,12}=0.272, \mathrm{p}=0.766$ ). A daily odor-evoked glomerular response was generated for each glomerulus by averaging all normalized same-odor trials within a single day to allow for pooling across subjects and statistical comparison of pooled glomerular responses. Averaged normalized glomerular responses on Pre1 were similar across groups. Additionally, glomeruli were distinguished as E5 Responsive (i.e., glomeruli that respond to E5 alone or E5 and one or more of the other odors) or Non E5 Responsive (i.e., glomeruli that do not respond to E5) for further analysis using the same criteria as above. Based on this classification, presentations of E5 will contain responses only from E5 Responsive glomeruli while presentations of non-conditioned odors will contain responses from both E5 Responsive and Non E5 Responsive glomeruli.

For behavioral analysis, freezing was measured from the onset of each stimulus presentation for a total of 60 seconds. In Tone-Shock experiments the freezing of all four tone presentations was averaged for each mouse.

\section{Results}

\section{Olfactory Aversive Conditioning Produces Fear Generalization to Multiple Odors}

For Experiment 1, we began by imaging glomerular responses to the panel of odors for two consecutive days, establishing baseline responses (Figure 2-1A). After the second imaging session, each mouse was placed into one of three training conditions: Odor only, Shock only, or Paired. Approximately 24 hours after conditioning, mice assessed for behavioral freezing to the CS (E5) as well as neutral, unlearned odors (E4, 

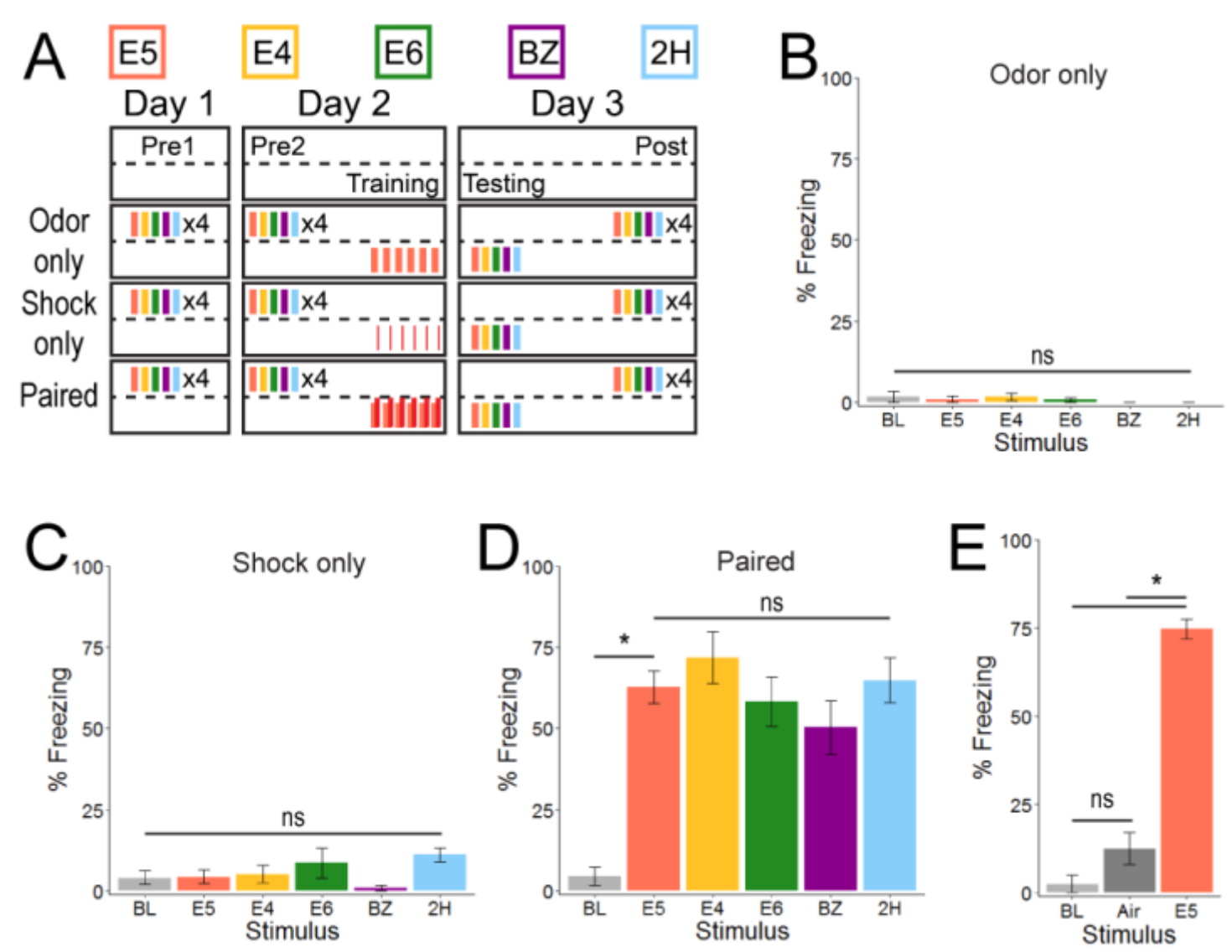

Figure 2-1. Olfactory aversive conditioning results in robust olfactory fear and generalization.

(A) Schematic detailing time course of experiments, the odors used (top), and paradigms for both imaging (above dotted line) and behavioral (below dotted line) experiments for each group. (B-D) 24 hours after training, mice were exposed to each of the five odors (E5, E4, E6, BZ, \& 2H) and freezing was measured. Odor only mice (B) and Shock only mice (C) did not learn to fear E5, as freezing was not significantly different than baseline (BL), and did not generalize freezing from E5 to other odors. Paired mice (D) froze significantly more to E5 than baseline, indicating acquired fear to the CS. Mice generalized fear across all other tested odors (freezing to other odors not significantly different from freezing to E5). Mice freeze specifically to odor cues (E). Data presented mean \pm SEM. 
E6, BZ, and 2H). Only mice in the Paired condition displayed strong, odor-evoked freezing $\left(\mathrm{F}_{5,24}=12.984, \mathrm{p}<0.0001, \eta^{2}=0.730\right)$, and only baseline freezing was significantly different from freezing to the CS $(\mathrm{p}<0.0001)$, suggesting the Paired training paradigm produces strong fear learning and broad behavioral fear generalization to all odors (Figure 2-1D). There was no difference in freezing for Odor only mice $\left(\mathrm{F}_{5,24}\right.$ $=0.635, \mathrm{p}=0.675, \eta^{2}=0.117$; Figure 2-1B); or Shock only mice across the different odors $\left(\mathrm{F}_{5,24}=1.933, \mathrm{p}=0.126, \eta^{2}=0.287\right.$; Figure 2-1C), indicating lack of fear.

\section{Olfactory Aversive Conditioning Potentiates Glomerular Responses in Awake Mice}

Following testing, mice underwent the final imaging session to assess the effect of conditioning on glomerular responses (Figure 2-2A-C). We first tested whether glomerular response amplitude changes over imaging session. Responses of Odor only ( $\mathrm{n}$ $\left.=570 ; \mathrm{F}_{1.930,1098.288}=2636.434, \mathrm{p}<0.0001, \eta^{2}=0.822\right)$, Shock only $\left(\mathrm{n}=508 ; \mathrm{F}_{1.895}\right.$, $\left.960.989=2665.888, \mathrm{p}<0.0001, \eta^{2}=0.840\right)$, and Paired mice $\left(\mathrm{n}=586 ; \mathrm{F}_{1.743,1019.743=}\right.$ $\left.907.598, \mathrm{p}<0.0001, \eta^{2}=0.608\right)$ changed over time. The glomerular responses of Odor only and Shock only mice decreased from Pre1 to Pre2 (Figure 2-3E, 2-3E', 2-3E') and were further reduced after training (Figure 2-3F, 2-3F', 2-3F'"), supporting the idea that glomerular responses decrease with increasing odor familiarity. Responses of Paired mice also decreased from Pre1 to Pre2 (Figure 2-3E, 2-3E'"'), but were significantly enhanced after training (Figure 2-3F, 2-3F"'). The observed glomerular response changes were also observed in the raw, unnormalized data (Figure 2-3E and 2-3F). Changes appear equal regardless of response amplitude, indicating the initial response amplitude does not impact the magnitude of experience-dependent response alterations.

\section{Olfactory Aversive Conditioning Does Not Alter Sniffing Rates in Awake, Head- Fixed Mice}

Though mice clearly demonstrated learned fear, as measured by freezing in the testing chamber, we did not observe any overt behavioral changes on the treadmill during the Post imaging session in response to odor presentations. This is likely due to the fact that mice largely remain still on the treadmill after the first few minutes of head-fixation and it is impossible to differentiate general lack of movement from fear-induced freezing as a result of odor presentations. While there was no overt behavioral response during imaging session, it is possible that awake mice increase their respiratory rate following training, which could alter the measured fluorescent signal (Blauvelt et al., 2013). We, therefore, examined whether altered breathing might be responsible for the observed changes reported above. MIF exhibited little to no change before and after odor onset (as measured by calculating the MIF for all respirations before odor onset and the MIF of the first four respirations after odor onset; Figure 2-4A, 2-4B, 2-4D, 2-4E, 2-4G, 2-4H), indicating no significant odor-evoked respiration changes in mice. Similarly, odorevoked MIF was not significantly different between groups before or after training (Pre2: $F_{2,73}=2.300, p=0.108 ;$ Post: $F_{2,73}=0.202, p=0.817$ ) and there was no effect of odor on odor-evoked MIF before or after training for any group (Figure 2-4C, 2-4F, 2-4I). 

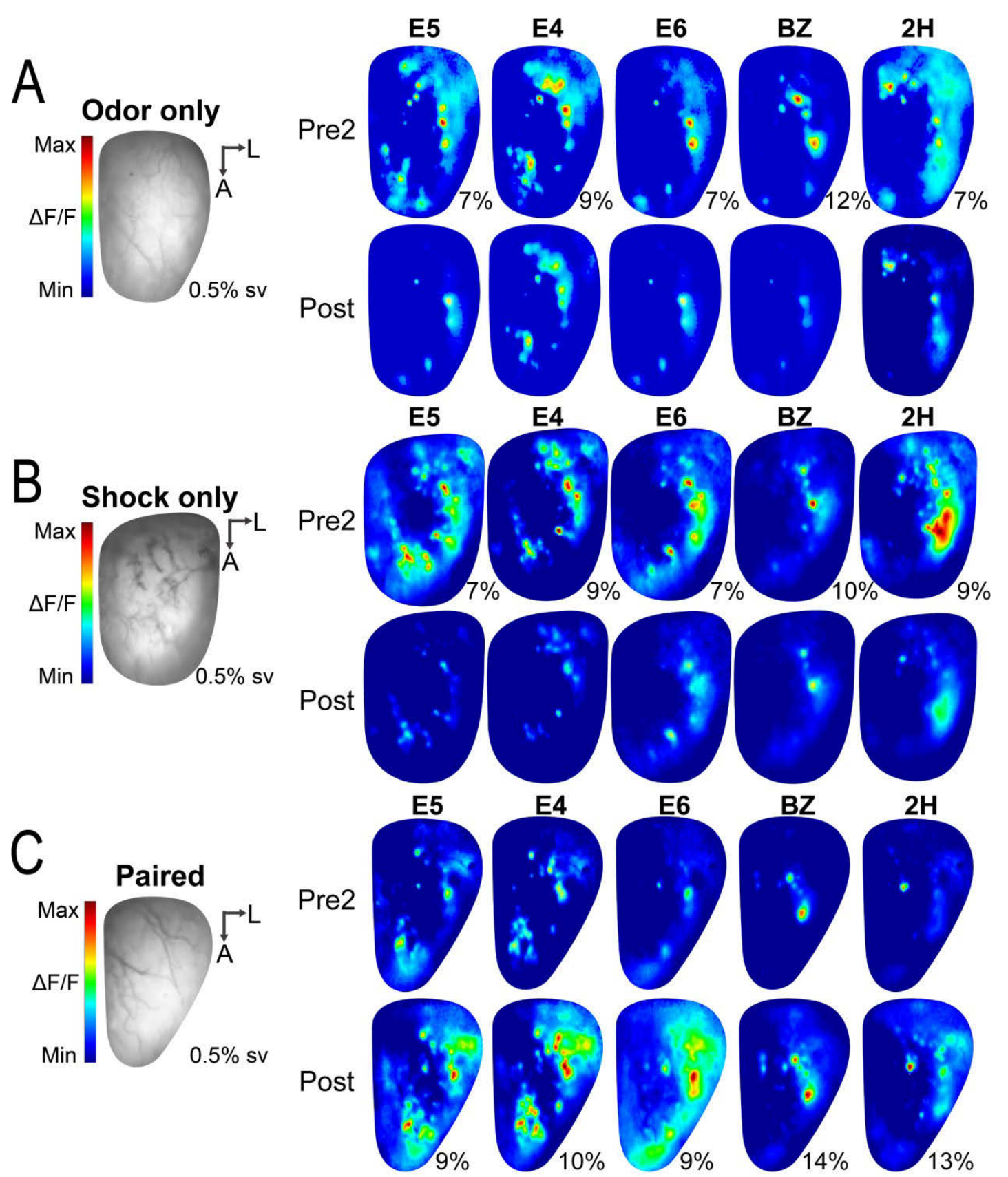

Figure 2-2. Olfactory aversive conditioning enhances glomerular responses. (A-C) Resting light intensity (RLI) frames and psuedocolored averaged Pre2 and Post glomerular response maps from representative Odor only (A), Shock only (B), and Paired (C) mice where the pseudocolor scale is based on the day with the maximum observed responses (Pre2 for Odor only and Shock only and Post for Paired) to avoid oversaturation of psuedocolored maps. The approximate value of the maximum observed responses $(\Delta \mathrm{F} / \mathrm{F})$ used for pseudocolor scale is listed to the right of each odor. While scaling color in this manner makes Pre2 responses appear significantly weaker in the Paired group, amplitude of Pre2 responses are similar across mice for each odor. The maximum observed responses are also statistically similar across groups (see Methods). 

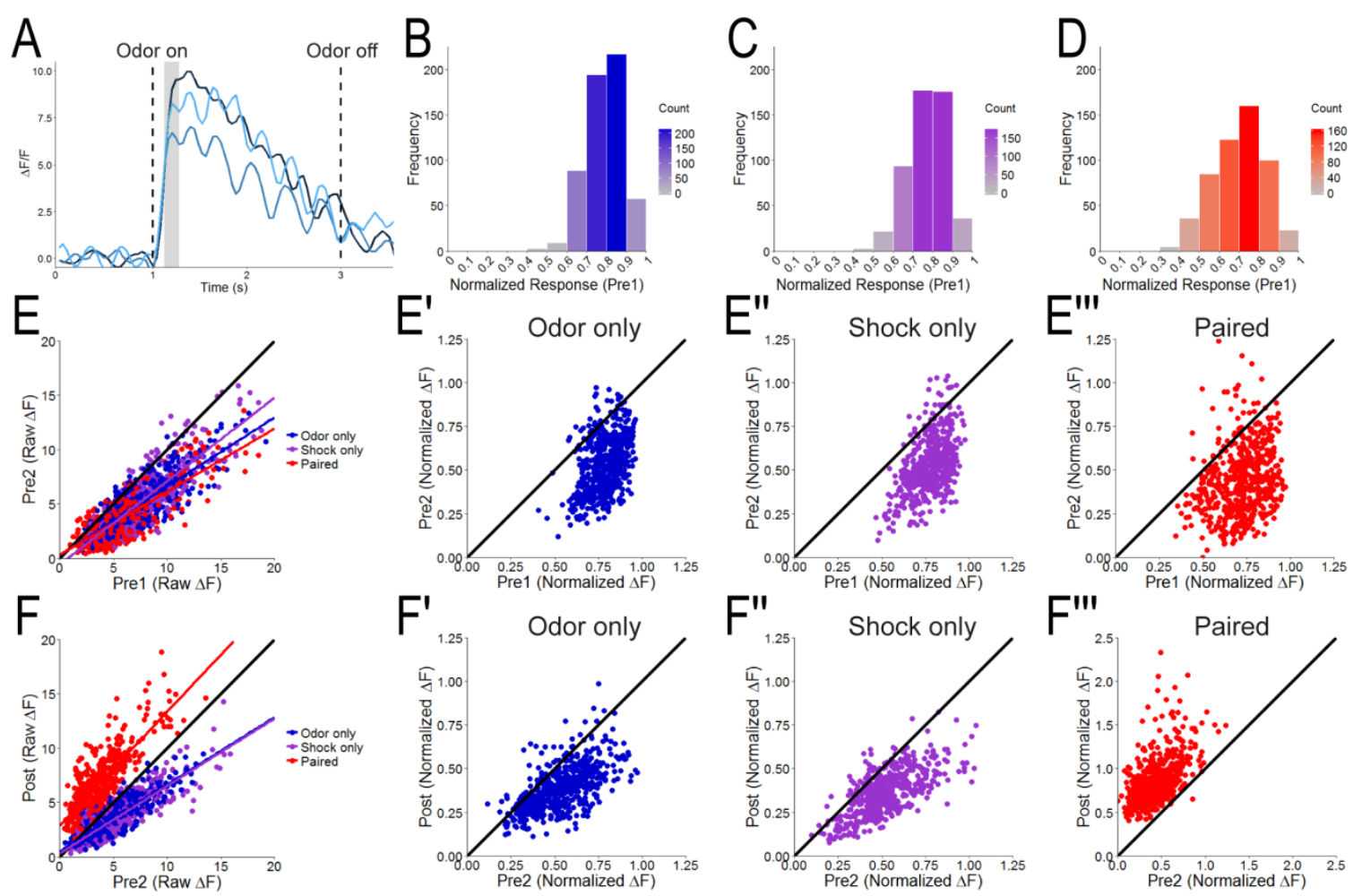

Figure 2-3. Post-training enhancements are independent of glomerular response amplitude.

(A) Example traces from representative glomerulus indicating Pre1 trial-to-trial variability in amplitude of response and extracted respiratory signal. Grey box illustrates 5 frames around the initial peak response used for analysis. (B-D) Histograms of normalized Pre1 responses illustrating range and frequency of glomerular responses for Odor only (B), Shock only (C), and Paired (D) mice. Responses on Prel exhibit unimodal distributions with an average normalized response of $\sim 0.8$ for all groups. (E) Scatterplot showing raw Pre1 (x-axis) corresponding raw Pre2 (y-axis) values for each recorded glomerulus from all Odor only (blue), Shock only (purple), and Paired (red) mice. Raw normalized responses display similar amplitudes and experience-dependent decreases across groups. Solid black line represents theoretical "no change" line. (F) Scatterplot showing raw Pre2 (x-axis) and corresponding raw Post (y-axis) values for each recorded glomerulus from all Odor only (blue), Shock only (purple), and Paired (red) mice. Scatterplots of normalized responses demonstrate similar changes to those of raw responses but allow for pooling across subjects. Normalized glomerular responses generally decrease from Pre1 to Pre2 for all groups, as evidenced by the majority of points falling below the no change line (E', E', E' "). Normalized glomerular responses also generally decrease from Pre 2 to Post for Odor only (F') and Shock only (F') mice while almost all glomerular responses increase from Pre2 to Post for Paired mice (F'", $)$, as evidenced by points falling above no change line. Importantly, almost all raw glomerular responses for Paired mice after training also fall above the no change line $(\mathrm{F}$, red). The post-training increase of raw glomerular responses appears linear, given the fit line is parallel to the no change line, indicating glomerular response enhancement is independent of glomerular response amplitude. 

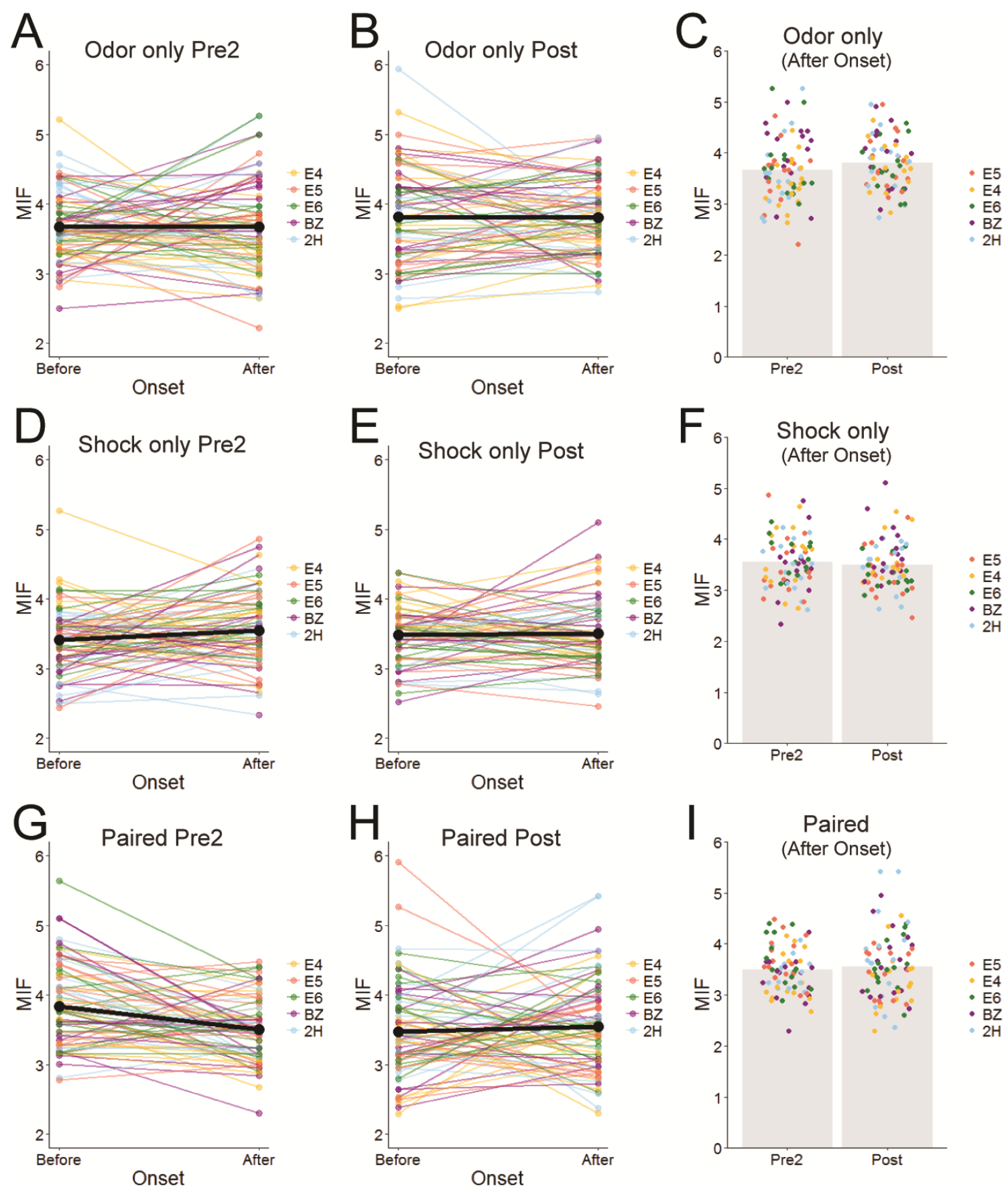

Figure 2-4. Mean instantaneous respiration frequence (MIF) is stable.

Extracted MIF from fluorescent trials (such as Figure 2-3A) falls in line with previously published data for awake, headfixed mice and ranges from $\sim 2-6 \mathrm{~Hz}$. Pre $2 \mathrm{MIF}$ is similar before and after odor onset for all Odor only (A), Shock only (D), and Paired (G) trials. Post MIF is stable before and after odor onset for all Odor only (B), Shock only (E), and Paired $(\mathrm{H})$ trials. Thick black lines represent calculated average MIF while colored lines represent every trial colored by odor. Odor-evoked MIF (after odor onset) is comparable on Pre 2 and Post for Odor only (C), Shock only (F), and Paired (I) mice. Importantly, MIF is not significantly higher or lower for any particular odor. 
During the post-training imaging session (Post), the difference between MIF before and after odor onset was not significantly different between groups $\left(\mathrm{F}_{2,72}=0.207\right.$, $\mathrm{p}=0.814$ ). Previous work suggests that awake mice typically only modulate sniffing to novel odorants, a behavior that rapidly declines as odors become familiar (Verhagen et al., 2007; Wesson et al., 2008; McAfee et al., 2016). Due to the design of our imaging paradigm, all odorants become familiar within the first imaging session, therefore it is not surprising that we fail to see sniffing modulation during Pre 2 or Post imaging session when odors are no longer novel. Furthermore, previous studies suggest rapid sniffing may attenuate, rather than augment, neural activity, and therefore fluorescent signal, in the OB (Verhagen et al., 2007), making increased sniff rates an unlikely cause of the observed post-training glomerular response enhancement. Altogether, the lack of altered sniffing after training suggests that recorded changes in the fluorescent signals are not due to differences in respiration between groups, but reflect real changes in glomerular activity as a result of experimental condition.

\section{Olfactory Aversive Conditioning Non-Specifically Potentiates Glomerular Responses to All Odors in Awake Mice}

We next examined whether the glomerular response changes were caused by a single odor by separately analyzing glomerular responses across time for each individual odor. Responses to all odors in the Odor only group exhibited similar decreases over time $\left(E 5: \mathrm{n}=133, \mathrm{~F}_{1.711,225.815}=579.243, \mathrm{p}<0.0001, \eta^{2}=0.814 ; \mathrm{E} 4: \mathrm{n}=136, \mathrm{~F}_{2,270}=\right.$ 1020.020, $\mathrm{p}<0.0001, \eta^{2}=0.883$; E6: $\mathrm{n}=82, \mathrm{~F}_{2,162}=507.476, \mathrm{p}<0.0001, \eta^{2}=0.862$; BZ: $\mathrm{n}=118, \mathrm{~F}_{1.597,186.899}=1165.399, \mathrm{p}<0.0001, \eta^{2}=0.909 ; 2 \mathrm{H}: \mathrm{n}=101, \mathrm{~F}_{2,200}=$ 253.07, $\mathrm{p}<0.0001, \eta^{2}=0.717$ ) and responses are significantly decreased from the preceding time point for all odors (Figure 2-5A). Analogous decreases across odors were observed in the responses from Shock only mice $\left(\mathrm{E} 5: \mathrm{n}=120, \mathrm{~F}_{1.637,194.820}=531.709, \mathrm{p}<\right.$ $0.0001, \eta^{2}=0.817 ; E 4: \mathrm{n}=145, \mathrm{~F}_{1.880,270.737}=771.225, \mathrm{p}<0.0001, \eta^{2}=0.843 ; \mathrm{E} 6: \mathrm{n}=$ $67, \mathrm{~F}_{1.618,106.807}=636.019, \mathrm{p}<0.0001, \eta^{2}=0.906 ; \mathrm{BZ}: \mathrm{n}=95, \mathrm{~F}_{1.602,150.625}=535.607, \mathrm{p}<$ $\left.0.0001, \eta^{2}=0.851 ; 2 \mathrm{H}: \mathrm{n}=81, \mathrm{~F}_{1.786,142.899}=518.47, \mathrm{p}<0.0001, \eta^{2}=0.866\right)$. Responses are significantly lower at each time point when compared to the preceding session across all odors (Figure 2-5B) as they are for the Odor only group. This implies additional exposure to E5 during the Odor only treatment or exposure to shock alone does not disproportionately affect some odors.

When exploring glomerular responses for different odors in the Paired group, we find all odors display the same pattern of decreased responses from Pre 1 to Pre 2 followed by robust potentiation at the Post time point $\left(E 5: \mathrm{n}=149, \mathrm{~F}_{1.767,261.512}=435.719, \mathrm{p}<\right.$ $0.0001, \eta^{2}=0.746 ; E 4: \mathrm{n}=154, \mathrm{~F}_{1.601,244.962}=330.582, \mathrm{p}<0.0001, \eta^{2}=0.684 ; \mathrm{E} 6: \mathrm{n}=$ $82, \mathrm{~F}_{1.389,112.483}=107.171, \mathrm{p}<0.0001, \eta^{2}=0.570 ; \mathrm{BZ}: \mathrm{n}=106, \mathrm{~F}_{2,210}=208.243, \mathrm{p}<$ $\left.0.0001, \eta^{2}=0.665 ; 2 \mathrm{H}: \mathrm{n}=95, \mathrm{~F}_{1.616,151.858}=90.222, \mathrm{p}<0.0001, \eta^{2}=0.490\right)$. Responses at Pre 2 are lower than those at Pre1 for all odors and Post responses are significantly higher than those at both Pre 2 for all odors (Figure 2-5C). In addition, Post responses are significantly elevated above those on Pre1 for all odors except BZ, where Post and Pre1 responses are statistically equal $(\mathrm{p}=0.346)$. 
A

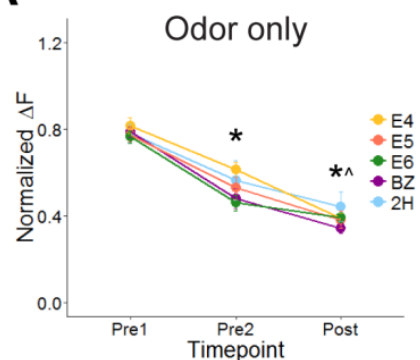

D

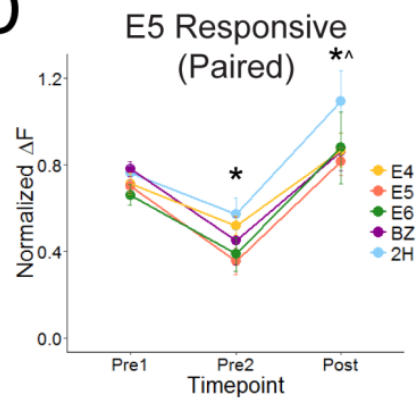

G

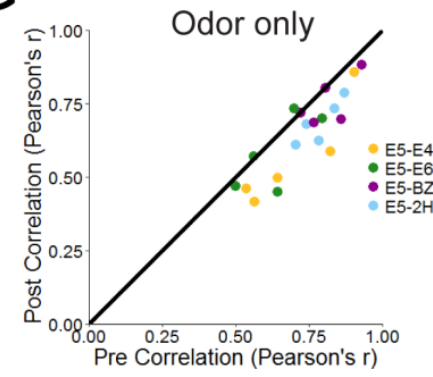

B

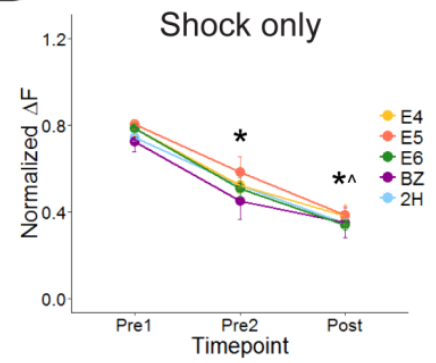

E Non E5 Responsive

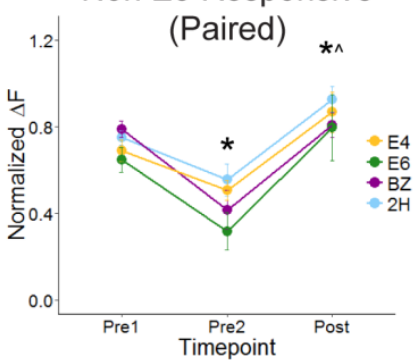

$\mathrm{H}$

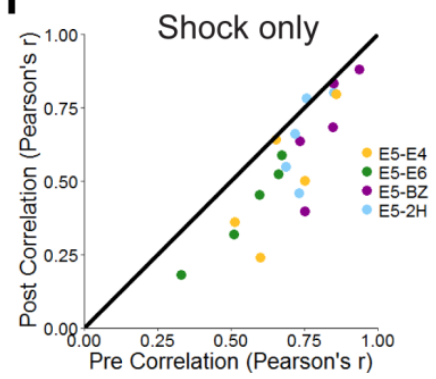

C
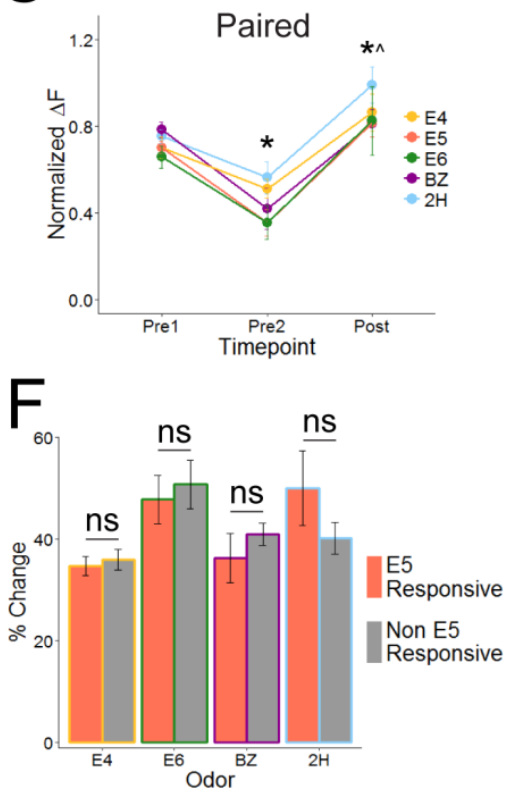

1

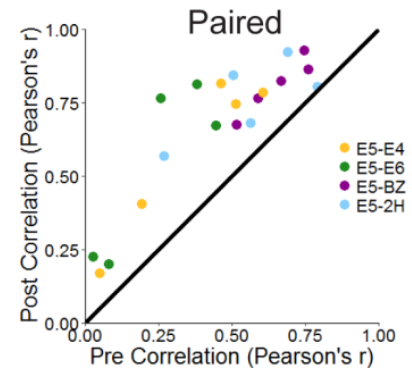

Figure 2-5. Enhanced responses are global, not odor- or glomerular-specific. (A-C) Normalized glomerular responses over time for each imaged odor. Responses for all odors continually decrease over time for Odor only (A) and Shock only mice (B). Glomerular responses to all odors of Paired mice (C) decrease before learning (from Pre1 to Pre2) followed by robust reinstatement of responses after learning (Post). The same learning-induced enhancement in Paired mice occurs in E5 Responsive (D) and Non E5 Responsive (E) glomeruli, indicating glomerular overlap does not play a significant role in learning-induced alterations (F). (G-H) Scatterplots showing the average correlation of spatial glomerular activation patterns between E5 and each of the neutral odors before (Pre2, x-axis) and after (Post, y-axis) for all Odor only (G), Shock only $(\mathrm{H})$, and Paired (I) mice. Solid black line represents theoretical "no change" line. Activation patterns of E5 and neutral odors decorrelate after training in both Odor only $(\mathrm{G})$ and Shock only $(\mathrm{H})$ mice but become more correlated after training in Paired mice (I). Data presented mean \pm sem. ${ }^{*} \mathrm{p}<0.001$ from Pre $1, \wedge \mathrm{p}<0.001$ from Pre2 
To confirm that these effects were not specific to the choice of CS odor, we trained a small number of mice to another odor in the set, BZ, and repeated the behavioral and imaging analysis. As with E5, we found similar global post-training glomerular enhancements to all odors when BZ was used as the CS. We also observed broad behavioral generalization to the other odors of the set as well as to a completely novel odorant acetophenone $\left(\mathrm{F}_{6,7}=11.649, \mathrm{p}=0.0024, \eta^{2}=0.909\right.$; freezing to $\mathrm{BZ}$ is significantly higher than baseline, $\mathrm{p}=0.004$, but not significantly different between $\mathrm{BZ}$ and any other odor, $\mathrm{p}<0.56)$. Together, this suggests that the olfactory fear conditioning non-specifically potentiates glomerular responses to all odors and leads to increased behavioral generalization, even to completely novel odors, regardless of odorant used as CS.

\section{Post-Training Enhancement of Neutral Odor Responses Is Independent of CS Overlap}

Many of the glomeruli measured were responsive to presentations of nonconditioned odors (E4, E6, BZ, and $2 \mathrm{H})$ as well as to presentations of E5. While glomerular enhancements were observed for all of the tested odors in the Paired group at the population level, it was not clear whether all glomeruli were enhanced similarly regardless of whether they responded to E5 and non-conditioned odors or only to nonconditioned odors. Therefore, we characterized each glomerulus as "E5 Responsive" or "Non E5 Responsive" and calculated the percent change from Pre2 to Post for each glomerulus, which allowed us to directly compare how much responses change based on whether glomeruli respond to the CS. When examining the 343 E5 Responsive glomerular responses to all different odors, only one was not enhanced after training, indicating E5 Responsive glomeruli are enhanced to all odors. Of the 243 Non E5 Responsive glomeruli, only one was not enhanced after training, indicating Non E5 Responsive glomeruli are also enhanced to all odors. While both E5 Responsive glomeruli and Non E5 Responsive glomeruli are enhanced for all tested odors, we next wanted to know whether there was a difference in the magnitude of enhancement for nonconditioned odors between the two classifications of glomeruli. Therefore, we directly compared the percent change from Pre2 to Post between E5 Responsive and Non E5 Responsive glomeruli for each non-conditioned odor. There was no significant difference in the Pre 2 to Post enhancement for glomerular responses to any of the non-conditioned odors in the Paired group (E4: $\mathrm{t}_{152}=-0.457, \mathrm{p}=0.0648$; $\mathrm{E} 6$ : $\mathrm{t}_{80}=-0.434, \mathrm{p}=0.666$; $\mathrm{BZ}$ : $\left.\mathrm{t}_{104}=-0.943, \mathrm{p}=0.348 ; 2 \mathrm{H}: \mathrm{t}_{93}=1.358, \mathrm{p}=0.178\right)$, signifying that the response properties of individual glomeruli responsive to E5 were not altered during training (Figure 2-5F), thereby causing the non-specific enhancement described above. Rather, olfactory fear learning induces a global enhancement of all glomeruli, independent of odorant and overlap with the CS. 


\section{Olfactory Fear Conditioning Increases Odor Representation Similarity Between CS and Neutral Odors}

We next investigated whether the changes in individual glomerular responses following training altered the overall representation of non-conditioned odors (E4, E6, $\mathrm{BZ}$, and $2 \mathrm{H}$ ) to be more or less similar to the conditioned odor (E5). To accomplish this, we generated the averaged glomerular response maps of each odor and asked how well correlated the spatial pattern of activation was between each of the non-conditioned odors and E5 before and after training. Our analysis suggests that non-conditioned odors are equally as correlated or modestly decorrelated with E5 after training for both Odor only (correlation values decreased an average of 0.0875 ) and Shock only mice (correlation values decreased an average of 0.1387; Figure 2-5G and 2-5H). In contrast, the patterns of spatial activity in response to non-conditioned odors are more similar to the response elicited by presentations of the CS after training for Paired mice than before (correlation values increased an average of 0.2153 ; Figure 2-5I).

\section{Olfactory Fear Learning Induces Long-Lasting Behavioral Fear and Enhanced Glomerular Responses}

In Experiment 2 we evaluated the duration of the post-learning effects. We repeated the Paired condition of the previous experiment but tested and imaged 72, rather than 24, hours after foot-shock training (Figure 2-6A). Mice exhibited robust freezing 72 hours after training $\left(\mathrm{F}_{5,18}=6.677, \mathrm{p}=0.001, \eta^{2}=0.650\right)$, where only baseline freezing was significantly different from freezing to the CS (E5), $\mathrm{p}<0.0009$. This indicates the odor-shock training paradigm produces broad, long lasting fear generalization across all odors (Figure 2-6B). The same mice also underwent awake imaging 72 hours after training (Post3) to characterize whether learning-induced glomerular enhancements were also long lasting. Again, global glomerular responses changed over time $\left(n=401 ; F_{1.529}\right.$, $\left.611.410=941.730, \mathrm{p}<0.0001, \eta^{2}=0.702\right)$. These mice displayed the characteristic response decrease from Pre1 and Pre2 and an enhancement of glomerular responses following training that was still visible $72 \mathrm{~h}$ later (Post3). Once again, this pattern is not driven by any particular odor $\left(E 5: \mathrm{n}=107, \mathrm{~F}_{1.642,174.104}=230.484, \mathrm{p}<0.0001, \eta^{2}=\right.$ 0.685; E4: $\mathrm{F}_{1.542,161.995}=222.538, \mathrm{p}<0.0001, \eta^{2}=0.679 ; \mathrm{E} 6: \mathrm{F}_{1.547,94.374=202.332, \mathrm{p}<}$ $0.0001, \eta^{2}=0.768 ; B Z: F_{1.705,78.445}=284.862, p<0.0001, \eta^{2}=0.861 ; 2 \mathrm{H}: F_{1.310,102.161}=$ $\left.225.059, \mathrm{p}<0.0001, \eta^{2}=0.743\right)$. In fact, responses to each odor on Post3 were significantly higher than responses for the same odor on Pre2 (Figure 2-6C; $\mathbf{p}<0.001$ ). Much like the initial enhancement, the sustained enhancement is not odor specific, indicating that olfactory fear learning globally increases glomerular responses in a longlasting manner. 


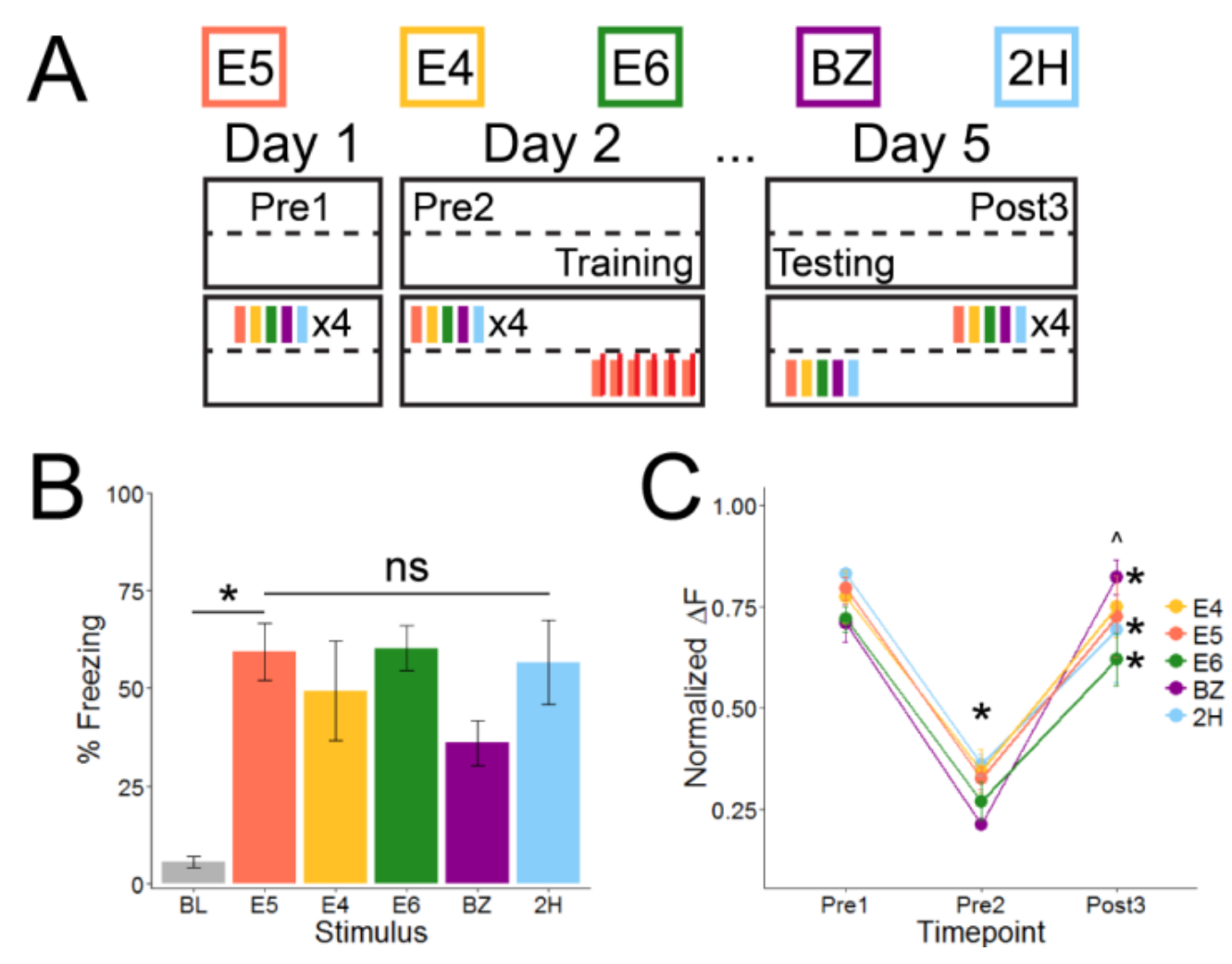

Figure 2-6. Olfactory fear learning induces long-lasting behavioral fear and glomerular enhancements.

(A) Schematic detailing time course of experiments, the odors used (top), and paradigms for both imaging (above dotted line) and behavioral (below dotted line) experiments. (B) 72 hours after training, mice were exposed to each of the 5 odors and freezing was measured. Mice froze significantly more to E5 than baseline, indicating acquired fear to the CS. Mice generalized fear across all other tested odors (freezing to other odors not significantly different from freezing to E5). (C) Glomerular responses to all odors significantly decrease from Pre1 to Pre2; however, even 72 hours after training, responses are significantly greater after learning (Post) than before (Pre2). Data presented mean \pm sem. ${ }^{*} \mathrm{p}<0.001$ from Pre $1, \wedge \mathrm{p}<0.001$ from Pre2 


\section{Anesthetized Mice Display Weaker Glomerular Enhancements and Suppression Following Olfactory Fear Learning}

In Experiment 3, we examined whether wakefulness modulates the olfactory learning-induced glomerular response profile. We repeated the Paired conditioning as in the first set of experiments but completed each of the imaging sessions in anesthetized, rather than awake, mice (Figure 2-7A). We confirmed olfactory fear learning in these mice by testing their awake behavioral freezing to each of the odors 24 hours following odor-shock conditioning (Figure 2-7B; $F_{5,18}=3.224, p=0.030, \eta^{2}=0.472$ ). Only freezing during the baseline minute is significantly different from freezing to $E 5(\mathrm{p}=$ 0.007), suggesting broad behavioral generalization similar to the above experiments even following repeated administration of anesthetics.

Mice in the anesthetized condition also display decreased glomerular responses from Pre1 to Pre2 (Figure 2-7B and 2-7C) followed by learning-induced glomerular enhancements $\left(n=292 ; F_{1.496,435.357}=68.702, p<0.0001, \eta^{2}=0.191\right)$; however, we noted the enhancement appeared reduced compared to the awake Paired group. A scatterplot demonstrated suppression of several glomeruli after training (Figure 2-7E), which occurred in all odors (E5: $\mathrm{n}=75, \mathrm{~F}_{1.492,110.435}=24.124, \mathrm{p}<0.0001, \eta^{2}=0.246$; $\mathrm{E} 4: \mathrm{n}=80, \mathrm{~F}_{1.315,103.906}=22.307, \mathrm{p}<0.0001, \eta^{2}=0.220 ; \mathrm{E} 6: \mathrm{n}=58, \mathrm{~F}_{1.297,73.934}=$ 11.469, $\mathrm{p}<0.0005, \eta^{2}=0.168 ; \mathrm{BZ}: \mathrm{n}=24, \mathrm{~F}_{2,46}=6.241, \mathrm{p}<0.004, \eta^{2}=0.213 ; 2 \mathrm{H}: \mathrm{n}=$ $\left.55, \mathrm{~F}_{1.409,76.098}=12.232, \mathrm{p}<0.0002, \eta^{2}=0.185\right)$. In addition, glomerular responses to both E6 and BZ were not significantly different from Pre2 to Post ( $\mathrm{p}=0.135$ and 0.231 , respectively). Out of the 292 glomeruli analyzed in the anesthetized mice, only $59.9 \%$ ( 175 total; $\mathrm{E} 5=47, \mathrm{E} 4=56, \mathrm{E} 6=34, \mathrm{BZ}=13,2 \mathrm{H}=25)$ were enhanced after training and $40.1 \%(117$ total; $\mathrm{E} 5=28, \mathrm{E} 4=24, \mathrm{E} 6=24, \mathrm{BZ}=11,2 \mathrm{H}=30)$ were decreased. Comparatively, in the awake Paired condition only 2 of the 586 glomeruli $(0.34 \%)$ analyzed exhibited lower responses after training. Also of note, we observed a smaller decrease in glomerular responses from Pre1 to Pre 2 in anesthetized mice than in awake mice, which supports previous work (Kato et al., 2012). This illustrates wakefulness modulates both passive experience- and learning-induced glomerular alterations and that anesthetized imaging in the context of experience-dependent glomerular transformations does not necessarily mirror that seen in an awake condition.

\section{Post-Training Glomerular Response Enhancements Are Independent of General Fear States}

One possible explanation for post-training glomerular response alterations in awake mice that differ from those observed in anesthetized mice is that the imaging paradigm requires continually presenting fear-inducing stimuli to the mice in order to assess post-training changes. It is possible that this repeated exposure induces a general fear state that enhances glomerular responses. Therefore, in Experiment 4 we devised a paradigm to image glomerular responses to odors in the presence and absence of a fear inducing stimulus (Figure 2-8A). Instead of fear conditioning Paired mice to an odor, we conditioned them to a $10 \mathrm{kHz}, 82 \mathrm{~dB}$ tone. Additionally, we split the Post time point into 


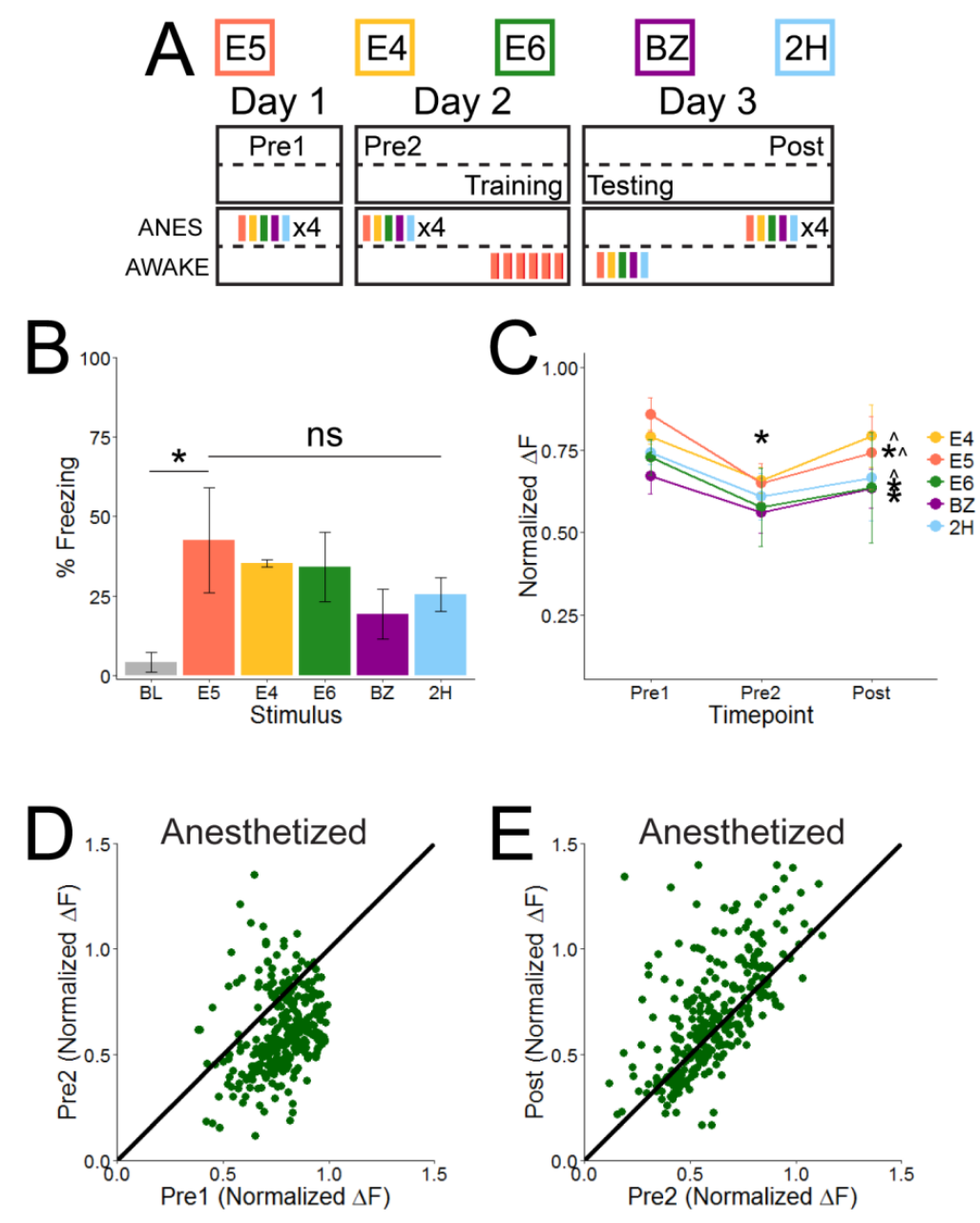

Figure 2-7. Learning-induced glomerular changes are variable in anesthetized mice.

(A) Schematic detailing time course of experiments, the odors used (top), and paradigms for both imaging (above dotted line) and behavioral (below dotted line) experiments. In this experiment mice are anesthetized (ANES) for all imaging sessions but awake during behavioral assays. (B) 24 hours after training, mice froze significantly more to E5 than baseline, indicating acquired fear and generalized fear across all other tested odors (freezing to other odors not significantly different from freezing to E\%). (C) Glomerular responses of anesthetized mice decreased from Pre1 to Pre2. After training (Post), only averaged responses of E5, E4, and $2 \mathrm{H}$ were significantly enhanced (relative to Pre2), while responses to E6 and BZ were not significantly different. (D\&E) Scatterplots showing normalized Pre1 (x-axis)/Pre2 (y-axis) responses (D) or Pre2 (x-axis)/Post (yaxis) responses (E) for each recorded glomerulus from Anesthetized mice. Solid black line represents theoretical "no change" line. Glomerular responses generally decrease from Pre1 to Pre2, as evidenced by the majority of the points falling below the no change line (D). On average, responses slightly increase from Pre2 to Post (E); however, of the 292 glomeruli analyzed in the anesthetized mice, only $59.9 \%$ were enhanced after training while $40.1 \%$ were suppressed. Data presented mean \pm sem. ${ }^{*} p<0.001$ from Pre1, $\wedge \mathrm{p}<0.001$ from Pre2 


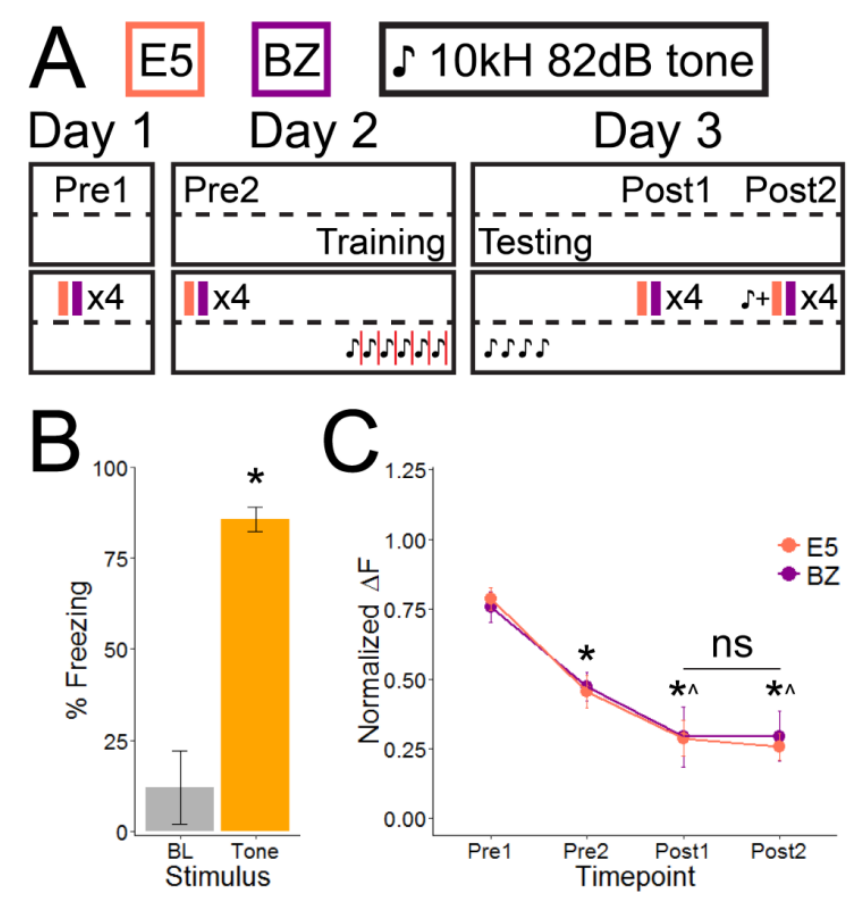

Figure 2-8. The expression of learning-induced glomerular response enhancements is independent of general fear states.

(A) Schematic detailing time course of experiments, the odors used (top), and paradigms for both imaging (above dotted line) and behavioral (below dotted line) experiments. (B) Tone-shock conditioned mice learn to fear the conditioned tone and freeze to it significantly more than baseline. (C) Odor-evoked glomerular responses of mice conditioned to fear a tone decreased from Pre 1 to Pre 2 and again from Pre 2 to Post 1. Importantly, there was no significant difference between odor-evoked glomerular responses during Pre1, when awake mice were imaged normally, and Post 2 , when we experimentally induced fear by preceding each odor imaging trial with a presentation of the fear-inducing tone. Data presented mean \pm sem. ${ }^{*} \mathrm{p}<0.001$ from Pre $1, \wedge p<0.001$ from Pre2 
two halves (Post1 and Post2). In the first half (Post1), we imaged odor responses normally; however, in the second half (Post2), we imaged odor responses immediately after a 10 s presentation of the conditioned tone.

Tone-shock conditioning produced robust freezing to presentations of the conditioned tone relative to baseline freezing in the first minute (Figure 2-8B; $\mathrm{n}=3, \mathrm{t}_{4}=$ $-6.650, \mathrm{p}<0.003)$. Glomerular responses significantly decreased across imaging sessions (Figure 2-8C; $\mathrm{n}=128 ; \mathrm{F}_{2.317,294.304}=729.000, \mathrm{p}<0.0001, \eta^{2}=0.852$ ) and the decrease was consistent across the two tested odors (E5: $\mathrm{n}=72, \mathrm{~F}_{2.215,157.276}=544.812, \mathrm{p}<$ $0.0001, \eta^{2}=0.885$; BZ: $\left.\mathrm{n}=56, \mathrm{~F}_{1.792,98.583}=239.018, \mathrm{p}<0.0001, \eta^{2}=0.812\right)$. All time points are significantly different from one another except Post 1 and Post $2(p=1.000$, for both odors). The lack of significant difference between recorded responses at Post1 (in absence of fear inducing tone) and Post2 (in presence of fear inducing tone) suggests a global fear state is not responsible for the augmented responses observed in odor-shock conditioned mice, but that the enhancement is likely due to fear learning itself.

\section{Global, but Not CS-Specific, Glomerular Enhancements Are Fear Learning Dependent}

Because the post-training enhancements could not be attributed to a global fear state that might simply strengthen all incoming sensory information, we next evaluated whether glomerular enhancements were dependent upon olfactory fear learning. In Experiment 5, we infused muscimol (MUSC) into the basolateral amygdala (BLA) 10 minutes prior to odor-shock training to inactivate the BLA, thus interrupting fear learning (Figures 2-9A and 2-10B). Mice that received infusions of vehicle (VEH) immediately before training demonstrate typical fear learning (Figure 2-9B; $\mathrm{n}=5, \mathrm{~F}_{5,24}=8.703$, $\mathrm{p}<$ $\left.0.0001, \eta^{2}=0.645\right)$. Mice freeze significantly more to E5 than baseline $(p<0.001)$ and generalize fear from E5 to E4, E6, and $2 \mathrm{H}$, but not to BZ ( $\mathrm{p}=0.020$, all others not significantly different from E5). On the other hand, MUSC mice did acquire learned fear (Figure 2-9C; $\mathrm{n}=5, \mathrm{~F}_{5,24}=1.107, \mathrm{p}=0.383, \eta^{2}=0.187$ ), confirming that infusions of MUSC into the BLA before training effectively block olfactory fear learning.

Not surprisingly, glomerular responses of VEH mice differed over the imaging sessions $\left(\mathrm{n}=596 ; \mathrm{F}_{1.384,823.392}=754.435, \mathrm{p}<0.0001, \eta^{2}=0.559\right)$, with a significant decrease in responses from Pre1 to Pre 2 and a significant increase from Pre 2 to Post (Figure 2-9D and 2-9F), similar to that of non-cannulated Paired mice. In general, glomerular responses of MUSC mice changed over time $\left(n=612 ; \mathrm{F}_{1.767,1079.568}=599.623\right.$,

$\left.\mathrm{p}<0.0001, \eta^{2}=0.495\right)$ and responses decreased from Pre 1 to Pre 2 with an additional, statistically significant decrease from Pre 2 to Post (Figure 2-9E and 2-9G; $p<0.001$ ). The lack of post-training enhancement in the MUSC group suggests these changes are learning-dependent; however, we noticed not all glomerular responses in the MUSC group were further decreased following odor-shock training. To determine whether responses to individual odors were differentially affected after training with infusions, we analyzed the responses of each odor individually. 
Figure 2-9. Generalized, but not CS-specific, glomerular enhancements are associative learning-dependent.

(A) Schematic detailing time course of experiments, the odors used (top), and paradigms for both imaging (above dotted line) and behavioral (below dotted line) experiments, including drug administration. (B\&C) Mice were exposed to all 5 odors 24 hours after training and freezing was measured. In mice receiving VEH infusions before training, presentations of E5 elicited significantly more freezing than baseline, indicating they learned to fear the CS. Additionally, VEH mice generalized fear to all odors, except BZ. In contrast, mice receiving MUSC infusions before training, did not freeze significantly more to presentations of E5 than baseline, indicating they did not learn. (D and E) RLI frames and psuedocolored averaged Pre2 and Post maps from representative VEH (D) and MUSC (E) mice, where the pseudocolor scale is based on the day with the maximum observed responses (Post for Vehicle, Pre2 for Muscimol for all odors except E5) to avoid oversaturation of psuedocolored maps. While scaling color in this manner makes Pre 2 responses appear significantly weaker in the Vehicle group, amplitude of Pre 2 responses are similar across mice for each odor. The approximate value of the maximum observed responses $(\Delta \mathrm{F} / \mathrm{F})$ used for pseudocolor scale is listed to the right of each odor. $(F \& G)$ Normalized glomerular responses over time for each imaged odor for all VEH (F) and MUSC (E) mice. Responses for all odors in both groups significantly decrease from Pre1 to Pre2. Responses to all odors are significantly increased after training in VEH mice. In contrast, only responses to presentations of E5 are enhanced in MUSC mice; all other odor responses are continually suppressed. In MUSC mice, the same post-training suppression of non-conditioned odors occurs regardless of whether glomeruli are E5 Responsive (H) or Non E5 Responsive (I). (J) Glomerular overlap does not affect the percent change of glomerular responses (Pre2 to Post) in VEH (top; with the exception of BZ) or MUSC (bottom) mice, indicating glomerular changes are odor- rather than glomerulus-specific (J). Data presented mean \pm sem. ${ }^{*} \mathrm{p}<0.001$ from Pre $1,{ }^{\wedge} \mathrm{p}<0.001$ from Pre2 

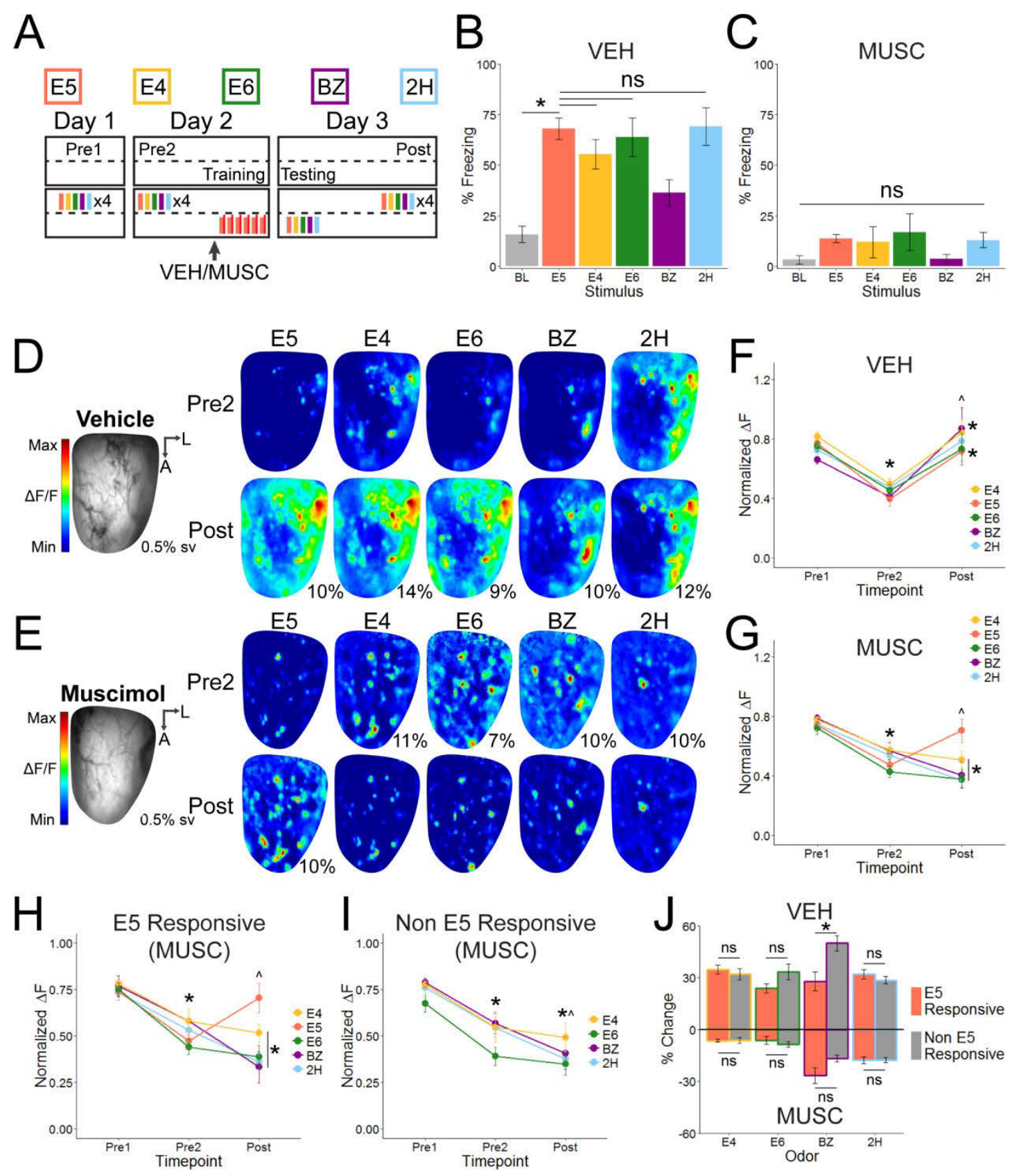


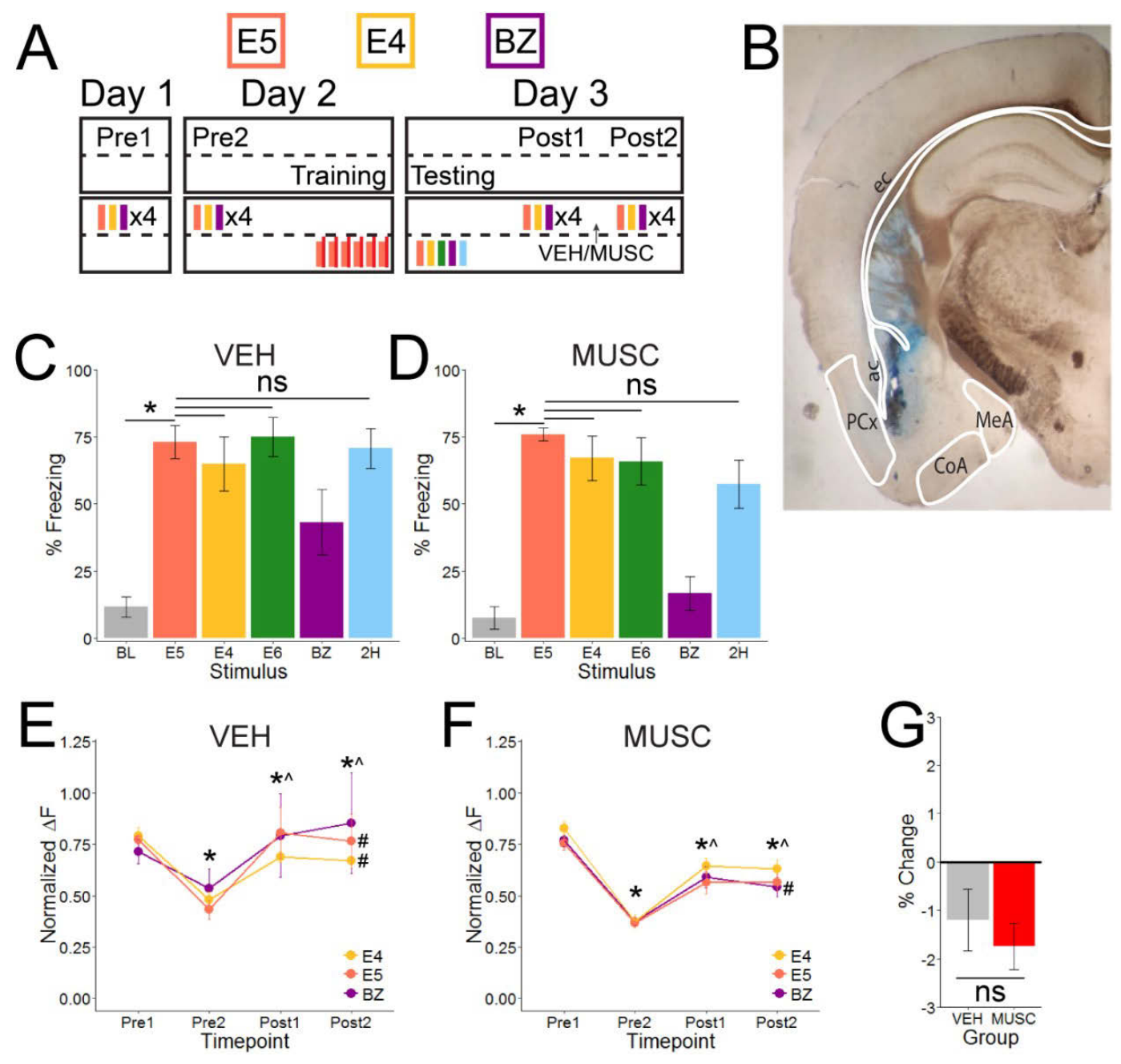

Figure 2-10. Amygdala inactivation during expression of learning does not impact glomerular responses.

(A) Schematic detailing time course of experiments, the odors used (top), and paradigms for both imaging (above dotted line) and behavioral (below dotted line) experiments, including drug administration. (B) Confirmation of BLA cannula placement and evaluation of possible MUSC spread. Abbreviations: Piriform cortex (PCx), cortical amygdala (CoA), medial amygdala (MeA), amygdalar capsule (ac), external capsule (ec). (C\&D) Freezing to all 5 odors was measured 24 hours after training and confirms that both groups of mice froze significantly more to the CS than baseline and generalized that fear to all odors except BZ. (E\&F) Averaged glomerular responses for both groups decreased significantly from Pre1 and Pre2, but were reinstated after learning (Post1). Infusions of either VEH or MUSC occurred between Post1 and Post2. Following infusions, both groups exhibited mixed profile responses, with some odor responses increasing, others decreasing, and some not changing significantly. Additional analysis quantifying the average \% change from Post1 to Post2 $(\mathrm{G})$ indicates no significant difference between groups, indicating BLA inactivation does not alter glomerular responses in a meaningful way. ${ }^{*} \mathrm{p}<0.001$ from Pre $1,{ }^{\wedge} \mathrm{p}<0.001$ from Pre $2, \# \mathrm{p}<0.001$ from Post1 
In the VEH group, all odors presented similar patterns of responsivity over the imaging sessions $\left(E 5: \mathrm{n}=140, \mathrm{~F}_{1.628,226.249}=393.528, \mathrm{p}<0.0001, \eta^{2}=0.739 ; \mathrm{E} 4: \mathrm{n}=\right.$ $141, \mathrm{~F}_{1.335,186.900}=190.665, \mathrm{p}<0.0001, \eta^{2}=0.577 ; \mathrm{E} 6: \mathrm{n}=91, \mathrm{~F}_{1.369,123.206}=119.941, \mathrm{p}$ $<0.0001, \eta^{2}=0.571 ; \mathrm{BZ}: \mathrm{n}=99, \mathrm{~F}_{1.206,118.164}=98.089, \mathrm{p}<0.0001, \eta^{2}=0.500 ; 2 \mathrm{H}: \mathrm{n}=$ $125, \mathrm{~F}_{1.654,205.090}=209.034, \mathrm{p}<0.0001, \eta^{2}=0.628$ ) with significantly lower Pre2 responses than Pre1, and significantly higher Post than Pre2 responses for all 5 odors (Figure 2-9F; $\mathrm{p}<0.001$ ). In contrast, not all odor responses in MUSC mice continue to decrease after training $\left(E 5: \mathrm{n}=160, \mathrm{~F}_{1.622,257.85}=195.242, \mathrm{p}<0.0001, \eta^{2}=0.551 ; \mathrm{E} 4: \mathrm{n}\right.$ $=156, \mathrm{~F}_{1.543,239.236}=284.548, \mathrm{p}<0.0001, \eta^{2}=0.647 ; \mathrm{E} 6: \mathrm{n}=89, \mathrm{~F}_{1.788,157.361}=234.108$, $\mathrm{p}<0.0001, \eta^{2}=0.727 ; \mathrm{BZ}: \mathrm{n}=85, \mathrm{~F}_{2,168}=179.119, \mathrm{p}<0.0001, \eta^{2}=0.681 ; 2 \mathrm{H}: \mathrm{n}=$ $\left.122, F_{2,242}=579.7, p<0.0001, \eta^{2}=0.827\right)$. While responses to all odors decrease from Pre1 to Pre 2 ( $\mathrm{p}<0.001$ for all five odors) at the population level, only glomerular responses to non-conditioned odors $(\mathrm{E} 4, \mathrm{E} 6, \mathrm{BZ}$, and $2 \mathrm{H})$ display further suppression from Pre2 to Post (Figure 2-9G; $p<0.001$ ), while glomerular responses to the conditioned odor, E5, are enhanced after odor-shock training $(\mathrm{p}<0.001)$. In fact, Post responses to E5 are not significantly different from those measured on Pre1 $(p=0.14)$, indicating full reinstatement of the initial E5 response after fear conditioning even in the absence of fear learning. These results illustrate that generalized, or global, glomerular enhancement is learning-dependent, while CS-specific enhancements do not require learning.

Again, many glomeruli responsive to E5 are also responsive to presentations of the other, non-conditioned odors. Further, responses to E5 were the only ones enhanced after training in MUSC mice. Therefore, we wanted to explore whether all "E5 Responsive" glomeruli might be enhanced, even when responding to neutral odors, but the effect obscured by averaging the responses of "E5 Responsive" with "Non E5 Responsive" glomeruli. We directly compared the effects of E5 overlap as conducted for Experiment 1. When mice received VEH infusions prior to odor-shock training, E5 glomerular responses increased $31.364 \% \pm 1.500 \%$ from Pre2 to Post. Glomerular overlap with E5 appeared to have no effect on the amount of observed post-training change for most of the non-conditioned odors (Figure 2-9H; E4: $\mathrm{t}_{139}=0.591, \mathrm{p}=0.555$; E6: $\mathrm{t}_{89}=-1.895, \mathrm{p}=0.061 ; \mathrm{BZ}: \mathrm{t}_{97}=-2.428, \mathrm{p}=0.017 ; 2 \mathrm{H}: \mathrm{t}_{123}=1.046, \mathrm{p}=0.298$ ) with only BZ exhibiting a significant difference between "E5 Responsive" and "Non E5 Responsive" in terms of percent change. E5 glomeruli of MUSC mice increased their responses $23.139 \% \pm 1.492 \%$ after training, while responses to all other odors decreased. Glomerular overlap with E5 did not impact the change from Pre 2 to Post for any of the non-conditioned odors (Figure 2-9I; E4: $\mathrm{t}_{154}=-0.153, \mathrm{p}=0.879$; $\mathrm{E} 6$ : $\mathrm{t}_{85.762}=0.797, \mathrm{p}=$ $\left.0.428 ; \mathrm{BZ}: \mathrm{t}_{83}=-1.537, \mathrm{p}=0.128 ; 2 \mathrm{H}: \mathrm{t}_{120}=-0.047, \mathrm{p}=0.963\right)$. This suggests a mechanism for odor rather than glomerulus-specific modulation in the OB following odor-shock training, even in the absence of fear learning (Figure 2-9J). Taken together, the results of this experiment provide evidence for two separate mechanisms that modulate OB glomerular responses. One is specific to the CS and does not require learning to regulate responses, while the second is learning-dependent and results in a global, or non-specific, gain across OB glomeruli. 


\section{Suppressing Fear Centers During Expression Does Not Suppress Learning-Induced Glomerular Enhancements}

Finally, in Experiment 6, we explored whether the global post-training glomerular enhancements could simply be due to amygdalar activation as a result of a fear state that increased sensory information in a modality-specific manner. Therefore, we investigated the effect of amygdalar inactivation on post-learning glomerular responses by infusing MUSC into the BLA (Figure 2-10B) halfway through the Post imaging session. This allowed for direct comparison of odor responses while the BLA was normally active or inactivated, respectively (Figure 2-10A). Both groups of mice were subjected to odorshock training, resulting in significant behavioral freezing (Figure 2-10C and 2-10D; VEH: $\mathrm{n}=5, \mathrm{~F}_{5,24}=9.056, \mathrm{p}<0.0001, \eta^{2}=0.654$; MUSC: $\mathrm{n}=5, \mathrm{~F}_{5,24}=17.437, \mathrm{p}<$ $\left.0.0001, \eta^{2}=0.784\right)$. Mice exhibit significantly higher freezing to E5 than baseline ( $p<$ 0.001, both groups), with broad generalization from E5 to all other odors in VEH mice ( $p$ $>0.065)$ and generalization from E5 to all odors except BZ $(p<0.001)$ in MUSC mice. These behavioral results are similar to the VEH results obtained in the previous experiment, which also contained mice that had bilateral cannula implantation in the BLA. Importantly, mice generalized fear from E5 to both E6 and $2 \mathrm{H}$ even though these are novel odors never experienced during previous imaging sessions

(Figure 2-10C and 2-10D). This is similar to earlier experiments where mice trained to BZ generalizing to the novel odorant acetophenone. Therefore, mice broadly generalized fear after odor-shock conditioning, even to the novel odorants E6 and $2 \mathrm{H}$.

Glomerular responses of VEH mice changed over time $\left(\mathrm{n}=367 ; \mathrm{F}_{1.477,540.494}=\right.$ 224.211, $\left.\mathrm{p}<0.0001, \eta^{2}=0.380\right)$, demonstrating a significant decrease in responses from Pre1 to Pre2 $(\mathrm{p}<0.001)$, rebound of glomerular responses from Pre2 to Post1 $(\mathrm{p}<$ 0.001), and a small decrease from Post1 to Post2, after VEH infusion into the BLA. Glomerular responses of MUSC mice changed similarly over the imaging sessions $(\mathrm{n}=$ $\left.379 ; \mathrm{F}_{2.151,813.016}=879.834, \mathrm{p}<0.0001, \eta^{2}=0.700\right)$, and display a significant suppression of responses from Pre1 to Post $2(p<0.001)$, enhancement from Pre2 to Post $1(p<0.001)$ and a significant decrease from Post 1 to Post2 $(p=0.002)$ after MUSC infusion. We noticed that all odors for both groups follow the same trend of decreasing from Pre1 to Pre2, with a rebound of the response from Pre2 to Post1, but that individual odors exhibit mixed response alterations between Post 1 and Post2. Therefore, we analyzed each odor separately over time (VEH E5: $\mathrm{n}=139, \mathrm{~F}_{1.359,187.490}=144.905, \mathrm{p}<$ $0.0001, \eta^{2}=0.512 ; E 4: \mathrm{n}=142, \mathrm{~F}_{2.212,311.887}=252.805, \mathrm{p}<0.0001, \eta^{2}=0.642 ; \mathrm{BZ}: \mathrm{n}=$ $86, \mathrm{~F}_{1.291,109.740}=19.612, \mathrm{p}<0.0001, \eta^{2}=0.187$; MUSC E5: $\mathrm{n}=145, \mathrm{~F}_{1.980,285.160}=$ $307.731, \mathrm{p}<0.0001, \eta^{2}=0.681 ; E 4: \mathrm{n}=145, \mathrm{~F}_{2.478,356.836}=494.151, \mathrm{p}<0.0001, \eta^{2}=$ 0.774; BZ: $\left.\mathrm{n}=89, \mathrm{~F}_{1.689,148.633}=145.011, \mathrm{p}<0.0001, \eta^{2}=0.622\right)$. In doing so, we discovered that responses to E5 and E4 were significantly decreased $(p<0.001$ and 0.02 , respectively), but $\mathrm{BZ}$ responses were not significantly different $(\mathrm{p}=0.116)$ from Post1 to Post2 in mice receiving VEH infusions (Figure 2-10E). In mice receiving MUSC infusions, responses to E5 and E4 were not significantly different ( $p=1.000$ and 0.126 , respectively), but BZ responses were significantly decreased $(p<0.001)$ from Post 1 to Post2 (Figure 2-10F). 
In light of the mixed response profiles from Post 1 to Post 2 in both groups, we decided to test whether the response changes between these imaging session halves was dependent upon experimental condition (infusion of either VEH or MUSC between the two halves). To do so, we calculated the percent change for each glomerulus from Post 1 to Post2. The response decrease from Post1 to Post2 was not significantly different between groups $\left(\mathrm{t}_{744}=0.679, \mathrm{p}=0.497\right.$, Mean $\pm \mathrm{SEM}$ : $\mathrm{VEH}=-1.202 \% \pm 0.634 \%$, MUSC $=-1.739 \% \pm 0.480 \%$ ), indicating inactivation of the BLA after learning did not reduce glomerular responses relative to VEH control responses (Figure 2-10G). Given MUSC infusion during the Post imaging session did not affect glomerular responses relative to controls, we surmise amygdalar activity during expression does not modulate OB glomerular responses, indicating OB enhancements are not a result of BLA activation following learning. While amygdalar activity does not appear to modulate OB responses in this case, it may still impact other brain regions to contribute to behavioral fear generalization.

\section{Discussion}

Pairing awake in vivo calcium imaging with behavior, we investigated the effect of classical olfactory aversive learning on glomerular odor responses. The results demonstrate that odor familiarity leads to reduced glomerular responses that are evident as early as the second day of odor exposure. These responses decrease over days, with learning producing significant and long-lasting enhancement of glomerular odor responses for the CS as well as neutral, non-conditioned odors. Enhancements are not specific to CS-responsive glomeruli, indicating global potentiation. Moreover, the spatial representations of non-conditioned odors become more correlated to the CS representation, possibly contributing to broad behavioral fear generalization by increasing perceptual similarity. Increased glomerular responses following fear conditioning are not caused by altered respiration or global fear states as a result of shock learning, nor can it be suppressed by inactivating the BLA after learning occurs. Chiefly, this study demonstrates two distinct mechanisms responsible for glomerular enhancements: an associative learning-independent mechanism, which supports CS-specific enhancements, and an associative learning-dependent mechanism, which promotes non-specific potentiation associated with generalization. To our knowledge, this is the first report of distinct mechanisms that mediate specific vs generalized response potentiation following classical fear learning.

These findings expand upon previous reports of learning-induced changes in olfactory regions in insects (Faber et al., 1999; Blum et al., 2009; Chen et al., 2015), rodents (Jones et al., 2008; Sevelinges et al., 2008; Barnes et al., 2011), and humans (Li et al., 2008). Recent imaging studies in anesthetized rodents demonstrate increased responses to the CS in OSNs, M/T cells, and inhibitory periglomerular cells (Fletcher, 2012; Kass et al., 2013; Kass and McGann, 2017). Our anesthetized experiment confirms these findings. In addition, we observed both increased and decreased post-training glomerular responses to the CS in our anesthetized experiment, as previously reported (Fletcher, 2012), while almost all glomeruli were significantly enhanced after training in 
awake mice. This likely reflects differences between anesthetized and awake conditions such as activity of intrabulbar inhibitory circuits or state-dependent centrifugal modulation (Tsuno et al., 2008; Blauvelt et al., 2013; Wachowiak et al., 2013; Rothermel and Wachowiak, 2014; Boyd et al., 2015), though it is also possible that robust expression of learning-induced glomerular plasticity requires wakefulness (Kato et al., 2012).

Importantly, most previous studies did not explore generalization, instead either restricting analysis to the $\mathrm{CS}$ or using a discriminative conditioning paradigm that results in CS-specific fear. Mice that learn to fear only the trained odorant display CS-specific enhancements (Kass et al., 2013). In contrast, classical training paradigms produce broad behavioral generalization (Pavesi et al., 2012; Kass and McGann, 2017), even to dissimilar odorants and completely novel odorants (Figure 2-10A, 2-10C, 2-10D). Our report is the first to examine the relationship between behavioral generalization and altered sensory representations in awake mice. Odor coding in the awake condition is characterized by narrowly tuned $\mathrm{M} / \mathrm{T}$ cell responses and enhanced inhibitory interneuron activity and centrifugal input (Kato et al., 2012; Wachowiak et al., 2013; Boyd et al., 2015). The combination of these factors likely plays an important role in olfactory processing, especially in the context of learning. Our awake training and imaging paradigms revealed broad behavioral generalization and global glomerular potentiation in the same mice, implying that $\mathrm{OB}$ plasticity reflects the associative learning event. In addition, we report increased representational similarity following olfactory conditioning. Given that spatiotemporal patterns of glomerular activity form the initial basis of odor identity, it is possible that such changes might increase perceptual similarity and bias downstream systems towards generalization. Together, these lines of evidence suggest that different training paradigms may generate global or specific sensory plasticity, which in turn could serve as an initial neural basis for generalized or specific behavioral responses.

\section{Potential Distinct Mechanisms of Experiment-Induced Glomerular Plasticity}

We demonstrate, in the absence of reinforcement, glomerular responses decrease across days. These findings support previous studies establishing that stimulus familiarity leads to reduced responses (Buonviso et al., 1998; Buonviso and Chaput, 2000; McNamara et al., 2008; Gdalyahu et al., 2012; Kato et al., 2012). While we did not explore the cause of the non-associative learning suppression, this likely arises from plasticity within OB inhibitory interneurons that are reduced under anesthesia, as this effect is not seen when imaging OSN glomerular responses (Kato et al., 2012). Interestingly, we detect less suppression in anesthetized than awake mice, which is consistent with this idea.

In terms of associative learning, we used MUSC to transiently inactivate the BLA during acquisition (Wilensky et al., 1999; Ribeiro et al., 2011) to prevent fear learning. It is possible that MUSC infusions also impact areas outside of the BLA, such as other portions of the amygdala or piriform cortex. While we assessed likely drug spread and 
believe the external and amygdalar capsules prevent lateral spread (Figure 2-10B), we cannot completely rule out the possibility that MUSC may also affect piriform cortex. However, the goal of MUSC injections into BLA was not to specifically assess the contribution of BLA to post-training glomerular enhancements but a method to block learning and evaluate whether enhancements were associative learning dependent, which was accomplished.

Even though MUSC mice did not acquire learned fear of E5, they exhibited augmented glomerular responses to E5. The fact that the CS-specific enhancements remain even when associative learning is blocked suggests that the first mechanism, which produces CS-specific enhancements, does not require associative fear learning. One possible explanation for this is experience-dependent structural changes within the glomerular layer itself. Fear conditioning can increase glomerular size (Jones et al., 2008); however, such structural changes are caused by increased number of OSN axons innervating glomeruli. We do not believe this drives our effect as the previous study trained mice over several days and weeks, allowing time for anatomical reorganization that our training paradigm likely does not allow. Alternatively, CS-specific plasticity could occur in the glomerular layer downstream of OSNs. Studies in pups reported odorspecific synaptic tagging in the OB glomerular and external plexiform layers as well as synaptic AMPA receptor insertion following odor experience (Modarresi et al., 2016), which could amplify glomerular responses. However, the enhancement we observed in the absence of learning was specific to E5, with no enhanced responses to other odors that activate those glomeruli. This suggests that the mechanisms responsible are not structural changes within OB neurons, as this should lead to glomerulus-specific enhancements. Instead, the mechanism underlying the odor-specific enhancements likely involves changes within OB circuitry encoding E5. Odor exposure and learning can decrease broad activation of granule cells (Woo et al., 1996), which should result in disinhibition of M/T cells and could be reflected in glomerular activity (Johnson et al., 1995; Huang et al., 2016). Further studies aimed at changes in specific cell types within the OB are needed to confirm this hypothesis.

The second mechanism is a global, non-specific enhancement of all glomeruli that is associative learning-dependent. Using an auditory fear paradigm in conjunction with OB imaging, we demonstrated that learning-induced enhancements are not a result of global fear states indiscriminately enhancing all incoming sensory information. However, it is possible that fear states modulate sensory processing in a modality-specific manner. Therefore, we additionally inactivated BLA by infusing MUSC to test whether suppressing fear centers during expression affects learning-induced glomerular enhancements. While we detected small differences in glomerular responsivity to some of the odors after MUSC infusion, those differences were no different than those exhibited by mice following VEH infusion (Figure 2-10F), suggesting BLA activity during expression does not modulate glomerular responses and is not the direct cause of learning-induced glomerular enhancements. Again, it is possible MUSC infusions affected more than just BLA and future studies should employ more spatially precise methods if they intend to parse out contributions of exact regions. Together, this evidence points to learning-induced changes in centrifugal modulation of $\mathrm{OB}$ responsivity from 
higher brain regions as a likely candidate. There is considerable feedback from cortical and neuromodulatory regions that can enhance OB responses to olfactory stimuli (Price and Powell, 1970; Haberly and Price, 1978; Mouret et al., 2009; Fletcher and Chen, 2010; Otazu et al., 2015; Linster and Cleland, 2016). Neuromodulatory systems can enhance representations of olfactory stimuli by acting on either local inhibitory interneurons or $\mathrm{M} / \mathrm{T}$ cells. For example, both acetylcholine and serotonin release in the OB and enhance M/T cells odor responses (Rothermel et al., 2014; Bendahmane et al., 2016; Brunert et al., 2016; Kapoor et al., 2016). Both of these systems are involved in fear learning (Pavesi et al., 2012; Bauer, 2015; Wilson and Fadel, 2017) and could serve as the mechanism behind global enhancements following fear conditioning.

\section{Potential Impact}

Importantly, OB glomerular responses constitute initial olfactory processing. M/T cells project odor information to cortical centers where it is integrated with other relevant information before ultimately driving behavioral responses. Generalized learning-induced enhancement of glomerular responses could serve to increase perceptual similarity of experienced odors, as evidenced by increased representational correlations, and thereby contribute to fear generalization. Previous reports in the olfactory system demonstrate olfactory discrimination learning decorrelates odor responses in areas of olfactory cortex (Kadohisa and Wilson, 2006), possibly decreasing perceptual similarity and making fine discrimination between odorants easier. In fact, decorrelated cortical representations of odor mixtures predict behavioral discrimination of those mixtures (Barnes et al., 2011), reinforcing the idea that olfactory representational similarity confers perceptual similarity in a way that influences behavior. Similar learning-induced effects are reported in other sensory and model systems, confirming that sensory learning alters sensory processing and correlates with behavior (Edeline et al., 1993; Mukai et al., 2007; Mundy et al., 2014; Smith et al., 2015). Taken all together, any learning-induced transformation, even at the earliest stages of processing, that increases or decreases the representational similarity of sensory stimuli may prime generalized or specific behavioral responses, respectively. Importantly, the amount of behavioral generalization does not perfectly match the magnitude of learning-induced glomerular potentiation. For example when E5 is the CS, mice occasionally freeze significantly less while demonstrating the largest glomerular potentiation to BZ (Figure 2-7B and 2-7F). There are two possibilities to explain such a phenomenon. While there is large enhancement to $\mathrm{BZ}$ responsive glomeruli, those that are BZ Responsive but non E5 Responsive are most enhanced (Figure 2-7J, top), possibly decreasing perceptual similarity and contributing to less behavioral generalization. It is also possible that the global potentiation serves on a more basic level to increase olfactory information being sent to cortical processing areas, which contribute to behavioral responses.

The present results emphasize that fear learning increases representational similarity of sensory stimuli at the earliest stages of processing, which may bias downstream fear regions towards generalization. As disrupted fear generalization is a hallmark of anxiety and trauma and stressor-related disorders (Cahill and Foa, 2007; 
Lissek et al., 2010; Lissek et al., 2014), understanding the mechanisms and brain regions underlying fear generalization may inform future treatments of this pathological behavior as well as our basic understanding of the mechanisms underlying long-term memory. 


\section{CHAPTER 3. CHOLINERGIC MODULATION OF OLFACTORY ASSOCIATIVE AVERSIVE MEMORY AND OLFACTORY BULB PLASTICITY}

\section{Introduction}

The olfactory bulb $(\mathrm{OB})$ receives significant input from neuromodulatory centers that release norepinephrine, serotonin, and acetylcholine (ACh) into the $\mathrm{OB}$, all of which can alter olfactory learning and associated plasticity (Wilson et al., 2004; Fletcher and Chen 2010; Ross and Fletcher, 2018b). In particular, the OB is innervated by cholinergic projection neurons from the horizontal limb of the diagonal band of Broca (Macrides et al 1981; Zaborszsky et al 1986). Interestingly, the cholinergic projection neurons terminate densely in the glomerular layer (Shipley and Ennis 1996) where odor information is first processed in the brain and represented in a spatiotemporal pattern of glomerular activation unique to each (Wachowiak and Cohen, 2001; Spors and Grinvald, 2002; Bozza et al., 2004; Mori et al., 2006; Fletcher et al., 2009; Storace and Cohen, 2017). Both nicotinic and muscarinic $\mathrm{ACh}$ receptor (AChR) subtypes are also widely expressed in the glomerular layer (Le Jeune et al., 1995; Castillo et al., 1999; Ghatpande and Gelperin, 2009; D'Souza and Vijayaraghavan, 2012) and have varied consequences on OB processing (Ravel et al., 1990; Elaagouby et al., 1991; Castillo et al., 1999; Liu et al., 2015; Smith et al., 2015; Case et al., 2017)providing distinct mechanisms by which ACh can modulate olfactory information.

Acetylcholine is crucial to olfactory learning, as disruption of ACh signaling affects olfactory learning across species (Ravel et al., 1994; Fletcher and Wilson, 2003; Mandairon et al., 2006; Chaudhury et al., 2009; Devore et al., 2012; Hellier et al., 2012; Williamson and Wright, 2013; Chan et al., 2017); however, relatively little is known regarding the extent to which cholinergic signaling affects aversive olfactory learning. Recent reports demonstrate that olfactory fear conditioning induces OB plasticity (Fletcher 2012, Kass \& McGann 2013, Kass \& McGann 2017, Ross and Fletcher 2018a), yet we currently lack a mechanistic understanding of the causes of such alterations. Modeling data demonstrates ACh during olfactory learning increases mitral cell (MC) synchrony and facilitates synaptic plasticity in piriform cortex (PCx), leading to enhanced learning (de Almeida et al., 2013). In line with this, systemically inhibiting cholinergic signaling during acquisition disrupts olfactory fear learning (Kroon and Carobrez, 2009; Silva et al., 2015) but does not impair olfactory perception (Doty et al., 2003; Pavesi et al., 2012) nor alter sensitivity to unconditioned, fear inducing stimuli (Anagnostaras et al., 1999). Together, this demonstrates ACh is required for olfactory fear learning, possibly by enabling plasticity required for learning associations between the conditioned stimulus (CS) and unconditioned stimuli during acquisition of fear learning. Considering $\mathrm{ACh}$ is required for olfactory fear learning and its widespread innervation of the olfactory system, its role in olfactory fear learning and plasticity presents an interesting target for further investigation.

Although it has been determined that olfactory fear learning is mediated by muscarinic, not nicotinic, AChRs (Pavesi et al., 2012), the systemic nature of muscarinic 
AChR (mAChR) antagonism makes it difficult to conclude whether the decreased fear learning was due to antagonism of mAChRs in olfactory regions or some other affected brain region, such as amygdala which also expresses mAChRs (Spencer et al., 1986; Bonner et al., 1988). Modeling suggests mAChRs regulate synaptic plasticity in PCx but also increase MC synchrony in the $\mathrm{OB}$, which could lead to enhanced PCx learning (Hasselmo and Barkai, 1995; de Almeida et al., 2013), making it important to establish whether $\mathrm{mAChRs}$ are required specifically in the OB during olfactory fear conditioning for learning to occur. Furthermore, there are two subtypes of mAChRs expressed widely in the $\mathrm{OB}$ and the use of broad $\mathrm{mAChR}$ antagonists makes it unclear which of the subtypes are necessary for olfactory fear learning. While cholinergic signaling through mAChRs appears to be necessary for fear learning, the exact role it plays in associative learning has yet to be determined.

Here we use a combination of behavioral pharmacology, optogenetics, and awake wide-field calcium imaging to characterize the role of OB ACh in olfactory fear learning and associated plasticity. In order to determine the extent to which OB muscarinic cholinergic signaling supports fear conditioning we directly infused scopolamine, a mAChR antagonist, into the OB during fear conditioning. When tested 24 hours later, mice in which mAChRs where inhibited during odor-shock pairing, exhibit significantly reduced learned fear to the CS. By infusing specific antagonists of different $\mathrm{mAChRs}$ directly into the $\mathrm{OB}$ during olfactory fear conditioning, we identify that activation of the $\mathrm{m} 1$ subtype, but not the $\mathrm{m} 2$ subtype, of $\mathrm{mAChRs}$ in the $\mathrm{OB}$ is necessary for acquisition of olfactory fear learning. Furthermore, we use mice expressing channelrhodopsin in cholinergic neurons to stimulate release of ACh specifically in the OB during olfactory fear conditioning and demonstrate that enhanced OB ACh can strengthen olfactory fear learning. This establishes that $\mathrm{OB}$ ACh can bidirectionally modulate the strength learning. We also subject mice to an odor investigation task under the influence of OB scopolamine and find that inhibition of mAChRs does not alter olfactory perception, and therefore cannot be the cause of suppressed learning. Finally, we pair wide-field calcium imaging of awake mice with systemic administration of SCOP during fear conditioning to determine the extent to which muscarinic cholinergic signaling supports fear conditioning-induced OB glomerular plasticity. At doses that completely block fear learning, $\mathrm{OB}$ plasticity is also suppressed, indicating muscarinic ACh signaling is essential during odor-shock pairing for associative learning-independent CS-specific glomerular enhancements. All together these results define a role for ACh in olfactory associative learning and OB glomerular plasticity.

\section{Methods and Materials}

\section{Animals}

A total of 105 mice were used. Olfactory bulb cannula experiments were performed using adult male and female C57BL6/J (Jax Stock no: 000664) mice $(\mathrm{n}=78)$. Optogenetic experiments were performed on adult male and female B6. Cg- $\mathrm{Tg}(\mathrm{Chat}-$ 
COP4*H134R/EYFP,Slc18a3)6Gfng/J (ChAT-ChR2+) and wild-type (ChAT-ChR2-) littermates (Jax Stock No: 014546) mice $(\mathrm{n}=15)$. Awake, wide-field calcium imaging was performed on adult male and female mice $(n=12)$ generated from crossing FVB/N$\mathrm{Tg}$ (Thy1-cre)1Vln/J (Jax Stock No: 006143) with either B6;129S-

Gt(ROSA)26Sortm38(CAG-GCaMP3)Hze/J (Jax Stock No: 014538) or B6J.CgGt(ROSA)26Sortm95.1(CAG-GCaMP6f)Hze/MwarJ (Jax Stock No: 024105), such that the resulting mice expressed the fluorescent calcium indicator GCaMP3 or GCaMP6f, respectively, under the Thy1-promotor. This enabled post-synaptic glomerular imaging of the dorsal OB surface. All experimental protocols were approved by the University of Tennessee Institutional Animal Care and Use Committee.

\section{General Methodology}

\section{Surgical procedures}

For all surgical procedures, mice were anesthetized under ketamine/xylazine $(100 / 10 \mathrm{mg} / \mathrm{kg}$, i.p.) and given carprofen $(5 \mathrm{mg} / \mathrm{kg}$, s.c. $)$ after depth of anesthesia was verified by tail pinch. Mice were secured in a stereotaxic device and maintained on a heating pad for the duration of the surgery. All mice were implanted with a stainless steel anchor screw in the parietal bone to help secure cannula/headbar/LED to the skull. Mice used for cannula experiments (Experiments 1 and 3) received stainless steel bilateral guide cannula (Plastics One; C235GS-5-2.0/SPC) implanted in the olfactory bulbs (Bregma: $4.2 \mathrm{~mm}$ anterior, $1 \mathrm{~mm}$ lateral on either side, $1 \mathrm{~mm}$ ventral). At the end of the surgery, a matching bilateral dummy and separate cap (Plastics One; C235DCS-5/SPC and 303DC/1B) were inserted into the guide cannula of cannulated mice. Mice used for optogenetic experiments were implanted with miniature blue LEDs (Osram; LBW5SN), following thinning of the bone overlying the OBs with a dental drill (Ogg et al., 2018). Mice used for imaging experiments were given at least two days to recover and all other mice were given at least one week to recover prior to experimentation.

\section{Drugs}

For cannulated mice, $0.5 \mu 1$ drug or vehicle infusions were delivered bilaterally at a rate of $0.125 \mu 1 / \mathrm{min}$. Infusion cannula were left in place for 2 minutes following delivery to allow for diffusion. Mice received one of the follow infusions either before training or before testing: non-selective muscarinic receptor antagonist scopolamine hydrobromide (SCOP; Sigma-Aldrich, cat. no: S0929), selective muscarinic $\mathrm{m} 1$ receptor antagonist pirenzepine dihydrochloride (PIR; Tocris Bioscience, cat. no: 1071), selective muscarinic $\mathrm{m} 2$ receptor antagonist AF-DX 116 (AFDX; Tocris Bioscience, cat. no: 1105), or an appropriate vehicle [Ringer's solution or dimethyl sulfoxide (DMSO; Sigma-Aldrich, cat. no: D8418)]. 


\section{Olfactory fear conditioning and testing}

Olfactory fear conditioning was carried out as previously described (Ross and Fletcher, 2018c). Briefly, all animals were trained in a single-day classical olfactory fear conditioning paradigm where six 10 s presentations of a single odor, ethyl valerate (E5; Sigma-Aldrich, cat. No: 290866) diluted to approximately 200ppm in mineral oil coterminated with a $0.6 \mathrm{~mA}, 0.5 \mathrm{~s}$ foot shock. All mice were allowed to acclimate to the training chamber for 10 minutes before training began. Twenty-four hours following training, mice were placed in a separate testing context and again given 10 minutes to acclimate to the chamber before they were assessed for behavioral fear to the CS. Fear was measured by behavioral freezing, a widely used measure of fear (Blanchard and Blanchard, 1969; Fanselow, 1980), which is characterized by cessation of voluntary movement. Testing consisted of two 20 s presentation of E5 (ITI = $3 \mathrm{~min}$ ), starting in the second minute of the test session. Freezing bouts, lasting a minimum of $2 \mathrm{~s}$, were calculated using FreezeFrame4 (Coulbourn Instruments), and binned into 60s segments, to be reported as percent of time spent freezing during the $60 \mathrm{~s}$ bin in which odor was present.

\section{Odor investigation}

Mice with bilateral OB cannula, were placed in a standard shoebox cage $(18.4 \mathrm{~cm}$ $\mathrm{W} \times 29.2 \mathrm{~cm} \mathrm{D} \times 12.7 \mathrm{~cm} \mathrm{H})$ devoid of bedding placed inside an open field chamber $(40 \mathrm{~cm} \mathrm{~W} \times 40 \mathrm{~cm} \mathrm{D} \times 35 \mathrm{~cm} \mathrm{H}$; Stoelting). Air or air odorized by $1 \%$ s.v. isoamylacetate (Sigma-Aldrich, cat. no: W205508) was constantly delivered to the open field chamber through tubing along one of the corners. The advantage of this paradigm is that it allowed for odor delivery without experimenter inference, mouse disruption, or visual/auditory cues that could result in unintended behavioral effects. A vacuum pulled air away through small holes in the chamber to prevent odor build-up. A video camera was positioned towards the side of the behavioral chambers and investigative behavior, defined as active sniffing with a raised head, was manually scored using ANY-maze (Stoelting). Ten minutes prior to being placed in the chamber, mice received infusions of either $1 \mathrm{mM}$ $\mathrm{SCOP}$ or VEH. Mice were given 10 minutes to acclimate to the chamber (with nonodorized air) before presentation of odorized air. Investigation behavior was scored for the final two minutes of the acclimation period and the first minute of the odor presentation.

\section{Optogenetic stimulation}

Prior to placement in the training chamber, the head-mounted LEDs were connected to a pulse generator using flexible, light-weight wires. Optogenetic stimulation occurred only during olfactory fear conditioning. The pulse generator delivered a $3 \mathrm{~s}$, $50 \mathrm{~Hz}$ train starting $7.5 \mathrm{~s}$ after odor onset, such that the stimulation spanned the final $2.5 \mathrm{~s}$ of the odor presentation and the $0.5 \mathrm{~s}$ foot shock. The stimulation parameters were based on previous laboratory experiments (Ogg et al., 2018). The genetic identity of mice (ChAT-ChR2- vs ChAT-ChR2+) was blind to experimenters until after the conclusion of the experiment. 


\section{Optical imaging}

Optical Imaging and quantification were carried out as previously described (Ross and Fletcher, 2018a). Briefly, awake mice underwent three consecutive days of headfixed imaging (Pre1, Pre2, and Post) on a treadmill allowing mice control of X/Y movement while preventing the head from moving. Pre1 and Pre2 imaging sessions took place prior to drug treatment and olfactory fear conditioning and were used to establish baseline OB glomerular odor responses to the trained odor, E5. The Pre 2 imaging session occurred at least 2 hours prior to fear conditioning and the Post imaging session occurred $\sim 2$ hours following behavioral testing. All imaging data was obtained with a Scientifica Slicescope equipped with a 4x objective (Olympus; 0.3 numerical apperture). The dorsal surface of the OB was illuminated with a LED light source centered at $480 \mathrm{~nm}$ for $5 \mathrm{~s} /$ trial and GCaMP signals were band-pass filtered with a Chroma emission filter (HQ535/50) and collected using a CCD camera at either 25Hz (NeuroCCD-SM256, Redshirt Imaging). Each imaging trial was 5 seconds in duration and consisted of an initial $2 \mathrm{~s}$ of no stimulus followed by $2 \mathrm{~s}$ of E5 presentation and a final 1s of no stimulus. Presentations of E5 were interspersed with no stimulus trials in which no E5 presentation occurred during the $5 \mathrm{~s}$ imaging trial. At least 4 E5 trials were collected each day.

\section{Detailed Methodology}

\section{Experiment 1}

Experiment 1a. Cannulated mice received infusions of SCOP (in Ringer's; $1 \mu \mathrm{M}$, $\mathrm{n}=9 ; 1 \mathrm{mM}, \mathrm{n}=7$; or $10 \mathrm{mM}, \mathrm{n}=4$ ) or vehicle (Ringer's, $\mathrm{n}=8$ ) prior to training to assess the role of $\mathrm{OB}$ muscarinic signaling in acquisition of olfactory fear learning.

Experiment 1b. Cannulated mice received infusions of SCOP (in Ringer's; 1mM, $\mathrm{n}=6$ ) or vehicle (Ringer's, $\mathrm{n}=8$ ) prior to testing to determine the extent to which OB muscarinic signaling is necessary for expression of learned fear.

Experiment 1c. Cannulated mice received infusions of PIR (in Ringer's; $1 \mathrm{mM}, \mathrm{n}$ $=7$ ), AFDX (in DMSO; $1 \mathrm{mM}, \mathrm{n}=7$ ), or vehicle (Ringers, $\mathrm{n}=7$ and DMSO, $\mathrm{n}=7$, respectively) prior to training to ascertain the role of specific OB muscarinic receptors in the acquisition of olfactory fear conditioning.

\section{Experiment 2}

ChAT-ChR2 + $(n=9)$ mice and their wildtype littermates (ChAT-ChR2-; $n=5)$ received optogenetic stimulation of $\mathrm{OB}$ cholinergic fibers during olfactory fear conditioning to evaluate the extent to which enhanced OB ACh during odor-shock pairing modulates olfactory fear learning. 


\section{Experiment 3}

Cannulated mice received infusions of $1 \mathrm{mM}$ SCOP (in Ringers; $\mathrm{n}=5$ ) or VEH ( $\mathrm{n}$ $=3$ ) before being placed inside a standard shoebox cage located in an open field chamber. Mice were given 600s to acclimate and the last 120s of acclimation were recorded and scored for investigative behaviors, defined as actively sampling by sniffing with a raised head. At the end of the $600 \mathrm{~s}$ acclimation phase, isoamylacetate added to the constantly circulating air stream to achieve $1 \%$ s.v. acetophenone. Investigation behavior was scored for an additional 1 minute. Each mouse was tested only once.

\section{Experiment 4}

Mice expressing GCaMP under the Thy1 promoter underwent imaging procedures as outlined above and received i.p. injections of SCOP (in saline; $10 \mathrm{mg} / \mathrm{kg}, \mathrm{n}$ $=4$ or $50 \mathrm{mg} / \mathrm{kg}, \mathrm{n}=4$ ) or an equal volume of vehicle (saline, $\mathrm{n}=4$ ) prior to training. Imaging mice were tested $\sim 24$ hours later and underwent the Post imaging session to assess the effects of muscarinic suppression on both behavioral expressions of fear and glomerular odor responses.

\section{Quantification and Statistical Analyses}

Olfactory fear learning was quantified as behavioral freezing, lack of voluntary movement, during the test session with FreezeFrame4 automated detection software (Coulbourn Instruments) in the 60s following odor presentation onset (Pavesi et al., 2012; Ross and Fletcher, 2018c). The CS, E5, was presented to each mouse two times during testing, and the freezing values for each epoch were averaged together to obtain a mean freezing score. The glomerular response imaging data was obtained as previously described (Ross and Fletcher, 2018a). Glomerular selections were obtained from a 2x2 pixel average taken at the center of each hand selected glomerulus. Glomerular traces obtained from the $2 \times 2$ pixel averaged were then corrected for photobleaching by applying an exponential subtraction fit through the first 2 non-stimulus seconds of each trial. Glomerular responses $(\Delta \mathrm{F})$ were calculated by obtaining the arbitrary fluorescence values during the 5 frames around the first odor-evoked respiratory peak and subtracting it from the 5 frames centered around the preceding non-odor evoked respiratory peak. $\Delta \mathrm{F}$ values were then divided by the arbitrary fluorescence values obtained from the first five frames of the imaging trial to obtain $\Delta \mathrm{F} / \mathrm{F}$ values. The mean daily glomerular response was collected for each animal by averaging the $\Delta \mathrm{F} / \mathrm{F}$ values for all $\mathrm{E} 5$ trials on a day for each glomerulus individually. The mean daily glomerular response for each mouse was then used to obtain a fold change value from Pre2 to Post for each glomerulus responsive to E5.

All statistical analyses were performed using Prism software (GraphPad, version 5.03) or SPSS (IBM, version 22). All data were subjected to testing for equal variances and normality. A one-tailed independent samples t-test was used to compare behavioral freezing between the two cannulated vehicle groups (Ringer's vs. DMSO), which 
revealed no significant behavioral difference ( $\mathrm{t} 12=0.4709, \mathrm{p}=0.6462, \mathrm{M}=57.960 \pm$ 5.527 and $54.78 \pm 3.868$, respectively) between the two vehicle controls. Therefore, Ringer's and DMSO vehicle controls were combined for analysis in Experiment $1 \mathrm{~b}$. ANOVAs were used for behavioral data in Experiments 1a, 1c while a one-tailed t-test was used for Experiments 1b, 2, and 3. An ANOVA was also used to compare fold change glomerular responses obtained from imaging in Experiment 4. Dunnett's t post hoc testing was performed where appropriate. All data are presented as mean $\pm \mathrm{SEM}$.

\section{Results}

\section{Muscarinic Neurotransmission Is Required in the OB During Acquisition for Olfactory Fear Learning}

Previous experiments demonstrate that muscarinic, but not nicotinic, neurotransmission is required during acquisition of fear learning (Pavesi et al., 2012); however, the use of systemic drug administration could not determine whether it is specifically required in the OB. Therefore, in Experiment 1a, cannulated mice received OB infusions of either VEH or various concentrations of SCOP $(1 \mu \mathrm{M}, 1 \mathrm{mM}$, or $10 \mathrm{mM})$ in order to test whether blocking muscarinic signaling specifically in the OB during acquisition affects fear learning. All mice were assessed for behavioral freezing to the conditioned stimulus (CS) 24 hours after training in order to measure fear learning (Figure 3-1A). VEH infused mice displayed robust behavioral freezing to the CS (mean freezing $=54.92 \% \pm 5.608)$. Infusions of SCOP prior to training significantly impeded fear learning $\left(F 3,24=19.724, p<0.0001, \eta^{2}=0.711\right)$. While the lowest SCOP dose $(1 \mu \mathrm{M})$ did not impact fear learning relative to $\mathrm{VEH}$ mice (mean freezing $=43.80 \% \pm 5.9$, $\mathrm{p}=0.309$ ), higher doses of $1 \mathrm{mM}$ and $10 \mathrm{mM}$ significantly decreased freezing (mean freezing $=8.31 \% \pm 4.7, \mathrm{p}=<0.0001$ and mean freezing $=2.55 \% \pm 1.7, \mathrm{p}<0.0001$, respectively). These results confirm that muscarinic neurotransmission is required for fear learning and establish that it is necessary specifically in the OB during acquisition.

\section{OB Muscarinic Neurotransmission Is Not Required for Expression of Olfactory Fear Learning}

Experiment 1a indicates that $\mathrm{OB}$ cholinergic signaling during acquisition is necessary for olfactory fear learning; however, the extent to which muscarinic neurotransmission is required during expression of fear learning is unclear. Therefore in Experiment $1 \mathrm{~b}$, we first subjected mice to olfactory fear conditioning and then infused either SCOP $(1 \mathrm{mM}$, based on the efficacy of $1 \mathrm{mM}$ SCOP in preventing fear learning when administered prior to training in Experiment 1a) of VEH prior to behavioral testing 24 hours after training (Figure 3-1B). Blocking muscarinic receptors via OB SCOP infusion (mean freezing $=47.27 \% \pm 5.1$ ) during testing had no effect on behavioral freezing relative to VEH controls (mean freezing $=47.66 \% \pm 5.2 ; \mathrm{t}_{12}=0.05283, \mathrm{p}=$ 0.4794), indicating $\mathrm{OB}$ muscarinic signaling is not required during the expression of a 

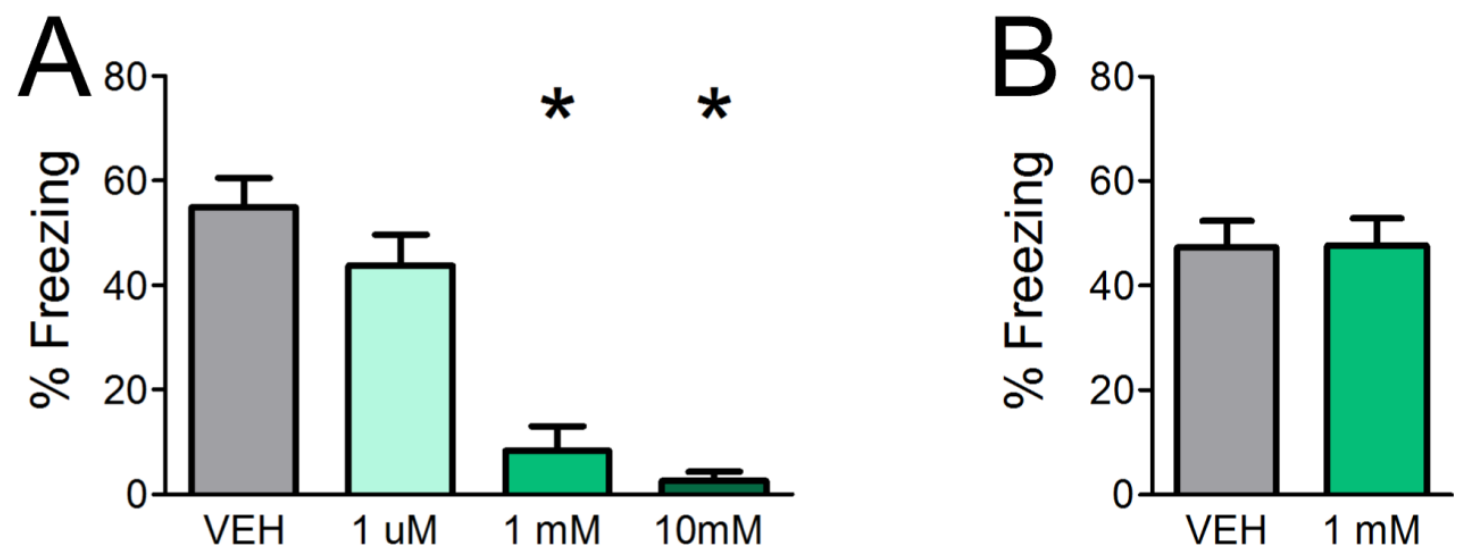

Figure 3-1. Direct OB application of scopolamine during fear conditioning impairs olfactory aversive fear learning but has no effect on expression of previously learned fear.

(A) Mice received infusions of vehicle (VEH) or different concentrations of scopolamine (SCOP; $1 \mu \mathrm{M}, 1 \mathrm{mM}$, or $10 \mathrm{mM}$ ), a non-selective antagonist of muscarinic acetylcholine receptors (mAChRs), through cannula directly into the olfactory bulbs prior to olfactory fear conditioning, in which a single odor (E5) was paired with mild foot shock. Mice were tested for behavioral freezing to the conditioned odor (E5) 24 hours later. Mice receiving infusions of $1 \mathrm{mM}$ and $10 \mathrm{mM}$ SCOP demonstrated reduced freezing relative to VEH controls, indicating impaired fear learning when mAChRs are blocked specifically in the olfactory bulbs. (B) Mice were first fear conditioned to E5. During testing, 24 hours after conditioning, mice received direct olfactory bulb infusions of VEH or $1 \mathrm{mM}$ SCOP. There is no significant difference in behavioral freezing between mice receiving infusions of VEH or $1 \mathrm{mM}$ SCOP, signifying antagonism of mAChRs during expression does not affect olfactory perception or behavioral displays of learned olfactory fear. Data presented as mean \pm SEM. $* \mathrm{p}<0.05$. 
previously learned olfactory fear.

\section{OB Muscarinic Neurotransmission, Specifically Through mAChR1, Is Required for Olfactory Fear Learning}

Scopolamine is a non-selective antagonist of muscarinic acetylcholine receptors (mAChRs). There are two types of mAChRs expressed widely in the OB, mAChR1 and mAChR2. In order to determine which of the receptor subtypes are necessary for fear learning, we infused specific antagonists for either mAChR1 (PIR) or mAChR2 (AFDX) or appropriate VEH in different mice prior to training. Both antagonists for Experiment $1 \mathrm{c}$ were delivered at a concentration of $1 \mathrm{mM}$ based on the efficacy of $1 \mathrm{mM}$ SCOP in Experiment 1a. Mice were tested for behavioral freezing, as a measure of learned fear, 24 hours after fear conditioning (Figure 3-2B). Mice receiving VEH infusions prior to training exhibiting robust freezing to the CS (mean freezing $=56.37 \% \pm 3.3$ ). As expected, inhibiting $\mathrm{mAChRs}$ blocked fear learning $\left(\mathrm{F}_{2,25}=12.111, \mathrm{p}=0.0002, \eta^{2}=\right.$ 0.4921); however, only infusions of PIR (mean freezing $=33.87 \% \pm 4.5$ ), not AFDX (mean freezing $=63.44 \% \pm 4.3$ ), decreased CS-evoked freezing relative to VEH mice (p $=0.001$ and $\mathrm{p}=0.367$, respectively). This suggests cholinergic signaling through $\mathrm{mAChR} 1$, but not $\mathrm{mAChR} 2$, during training is required for olfactory fear learning.

\section{Stimulating Release of OB ACh During Odor-Shock Pairing Strengthens Olfactory Fear Learning}

Optogenetic OB stimulation has previously been shown cause behavioral dishabituation of ChAT-ChR2 + but not wild-type (ChAT-ChR2-) littermates (Ogg et al., 2018), consistent with the idea that the stimulation paradigm induces release of $\mathrm{ACh}$ into the $\mathrm{OB}$. Given that $\mathrm{ACh}$ is necessary during conditioning in order to acquire olfactory fear, we next tested whether supplemental OB ACh could augment fear learning. In Experiment 2 we optogenetically stimulated release of OB ACh specifically during each of the six odor-shock pairings and tested behavioral freezing 24 hours later (Figure 3-3). ChAT-ChR2 + mice displayed augmented freezing (mean freezing $=61.04 \% \pm 5.0$ ) during testing relative to ChAT-ChR2- mice (mean freezing $=43.9 \% \pm 6.3 ; \mathrm{t}_{12}=2.077, \mathrm{p}$ $=0.030$ ). These results suggest that increasing $\mathrm{OB}$ acetylcholine during acquisition of olfactory fear learning can enhance the strength of the learned association.

\section{Olfactory Investigative Behavior Is Not Affected by Direct OB Antagonism of mAChRs}

While previous reports indicate mice lacking certain $\mathrm{mAChRs} \mathrm{exhibit} \mathrm{normal}$ basic olfactory investigation (Chan et al., 2017) and systemic administration of the mAChR antagonist SCOP does not impair olfactory perception (Doty et al., 2003; Pavesi et al., 2012), it is unclear whether direct OB application of mAChR antagonists affects olfactory behaviors. In order to determine whether the observed learning impairments 

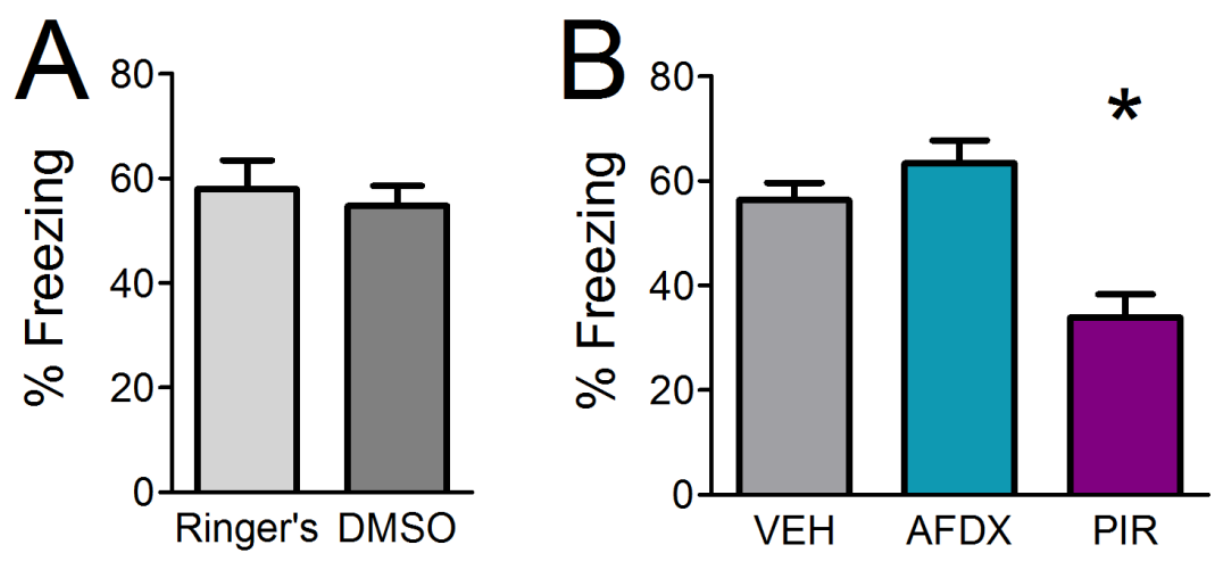

Figure 3-2. Inhibition of mAChR1, but not mAChR2, decreases behavioral freezing to the conditioned odor.

Mice received direct olfactory bulb infusions of vehicle (VEH, either Ringer's or DMSO) or AFDX, a specific antagonist of the $\mathrm{m} 2$ subtype of $\mathrm{mAChRs}$, or PIR, a specific antagonist of the $\mathrm{m} 1$ subtype of mAChRs, prior to olfactory fear conditioning. The mice were then tested for behavioral freezing 24 hours later. (A) Mice receiving Ringer's VEH and those receiving DMSO VEH prior to conditioning do not exhibit different freezing during testing, indicating no difference in learning as a result of the different VEH conditions. (B) There is no significant different in freezing between VEH mice (combined Ringer's and DMSO) and those receiving infusions of the mAChR2 antagonist AFDX; however, mice treated with PIR before conditioning display reduced freezing relative to $\mathrm{VEH}$ mice, suggesting $\mathrm{mAChR} 1 \mathrm{~s}$ specifically are required for appropriate acquisition of olfactory fear. Data presented as mean \pm SEM. ${ }^{*} \mathrm{p}<0.05$. 


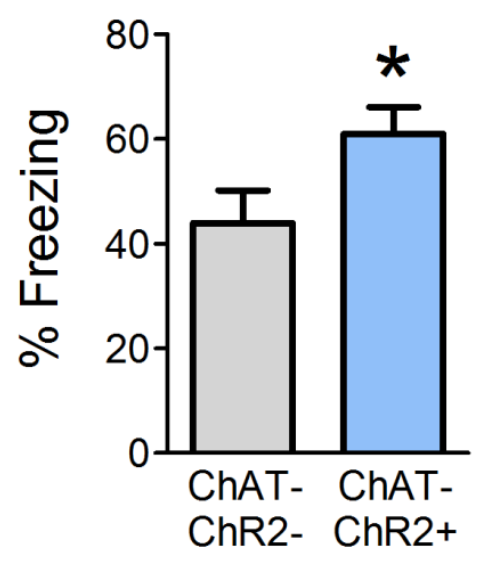

Figure 3-3. Enhanced OB acetylcholine during odor-shock pairing augments olfactory fear learning.

Mice were surgically implanted with a miniature LEDs directly above the olfactory bulbs. During olfactory fear conditioning all mice received light stimulation at the end of each of the six odor-shock pairing.s Positive ChAT-ChR2 mice express channelrhodopsin in cholinergic cell populations, such that light stimulation should induce release of ACh in the OBs during odor-shock pairing. When tested 24 hours later, ChAT-ChR2+ mice freeze significantly more than ChAT-ChR2- mice, which do not express channelrhodopsin in cholinergic cell populations and should experience no additional $\mathrm{ACh}$ release in the OBs as a result of light stimulation. This suggests that increasing OB ACh during olfactory fear conditioning can strengthen fear learning. Data presented as mean \pm SEM. $* \mathrm{p}<0.05$. 
were a result of reduced olfactory perception following mAChR antagonism, we subjected mice to an olfactory investigation task in Experiment 3 to assess olfactory function. In Experiment 3 (Figure 3-4), OB administration of SCOP (1mM) did not affect investigation of an odorized ball relative to $\mathrm{VEH}\left(\mathrm{t}_{6}=1.483, \mathrm{p}=0.0943\right)$. Mice spent the same amount of time performing investigatory behaviors during odor presentation regardless of whether they had received an OB infusion of VEH or SCOP (investigation time $=29.9 \pm 2.2 \mathrm{~s}$ and $33.4 \pm 1.3 \mathrm{~s}$, respectively). Together these experiments indicate that SCOP, administered either systemically or directly in the OBs, does not induce anosmia nor altered olfactory perception.

\section{Muscarinic Signaling Is Required for Olfactory Fear Learning-Induced Glomerular Enhancements}

Previous work demonstrates olfactory fear learning enhances glomerular responses to the CS (Ross and Fletcher, 2018a) and that systemic administration of SCOP prior to training is sufficient to decrease fear learning (Pavesi et al., 2012). Given that OB cholinergic signaling through $\mathrm{mAChRs}$ appears to be required for olfactory fear learning and that glomerular CS enhancements exist even after learning is blocked by silencing the basolateral amygdala (Ross and Fletcher, 2018a), we aimed to test whether muscarinic signaling might also be required for enhanced CS glomerular responses. In Experiment 1, we used systemic administration of SCOP $(10 \mathrm{mg} / \mathrm{kg}$ or $50 \mathrm{mg} / \mathrm{kg})$ or VEH combined with awake, wide-field imaging to test the effects of mAChR antagonism on fear learning and the associated glomerular plasticity.

As expected, systemic SCOP administration prior to training during Experiment 3 disrupted olfactory fear learning (Figure 3-5A; $F_{2,11}=26.007, p=0.0002, \eta 2=0.8525$ ). $\mathrm{VEH}$ mice display robust freezing to presentations of the CS during testing (mean freezing $=64.95 \% \pm 3.204)$. Both $10 \mathrm{mg} / \mathrm{kg}$ (mean freezing $=25.19 \% \pm 9.646$ ) and $50 \mathrm{mg} / \mathrm{kg}$ (mean freezing $=5.369 \% \pm 1.699) \mathrm{SCOP}$ doses were sufficient to decrease freezing relative to $\mathrm{VEH}$ mice $(\mathrm{p}=0.002$ and $\mathrm{p}=0.0001$, respectively) and the highest dose appears to completely block fear learning. After testing the same mice underwent a final imaging session to assess the effect of $\mathrm{mAChR}$ antagonism during odor-shock pairings on glomerular responses (Figure 3-5B and 3-5C). Fear learning induced robust glomerular enhancements in VEH mice, resulting in a $\sim 2$.4-fold increase in glomerular responses $(n=103 \mathrm{CS}$ responsive glomeruli) after training, which is consistent with previous reports of learning-induced glomerular enhancements (Ross and Fletcher 2018a). Overall, blocking mAChRs during training with SCOP altered olfactory fear learning-induced glomerular enhancements $\left(F_{2,286}=555.951, p<0.0001, \eta 2=0.7954\right)$.

Mice receiving $10 \mathrm{mg} / \mathrm{kg}$ SCOP displayed significantly decreased behavioral freezing relative to VEH mice, as well as significantly less learning-induced enhancement of glomerular responses after training ( $\mathrm{p}<0.0001)$, only $\sim 1.4$ fold $(\mathrm{n}=101 \mathrm{CS}$ responsive glomeruli). The $50 \mathrm{mg} / \mathrm{kg}$ dose of SCOP, which was effective at completely blocking olfactory fear learning, suppressed CS-specific glomerular enhancements relative to $\mathrm{VEH}$ controls $(\mathrm{p}<0.0001)$. Glomerular responses were $\sim 1.9$-fold lower after 


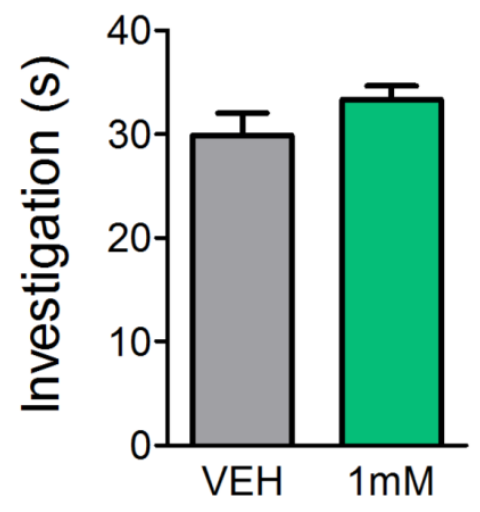

Figure 3-4. Antagonism of $\mathrm{OB}$ mAChRs does not alter olfactory driven behaviors. Mice underwent an olfactory investigation paradigm inside an open field chamber to determine whether the $\mathrm{mAChR}$ antagonist, scopolamine (SCOP), alters olfactory behaviors. Cannulated mice received direct OB infusions of either $1 \mathrm{mM} \mathrm{SCOP}$ or VEH. Time spent performing olfactory investigative behaviors was then scored in response to uncued odor presentations. Mice receiving OB SCOP did not differ from those receiving $\mathrm{VEH}$ in terms of time spent investigating. This demonstrates non-specific antagonism of mAChRs does not alter olfactory driven behaviors or induce temporary anosmia. Data presented as mean \pm SEM. 

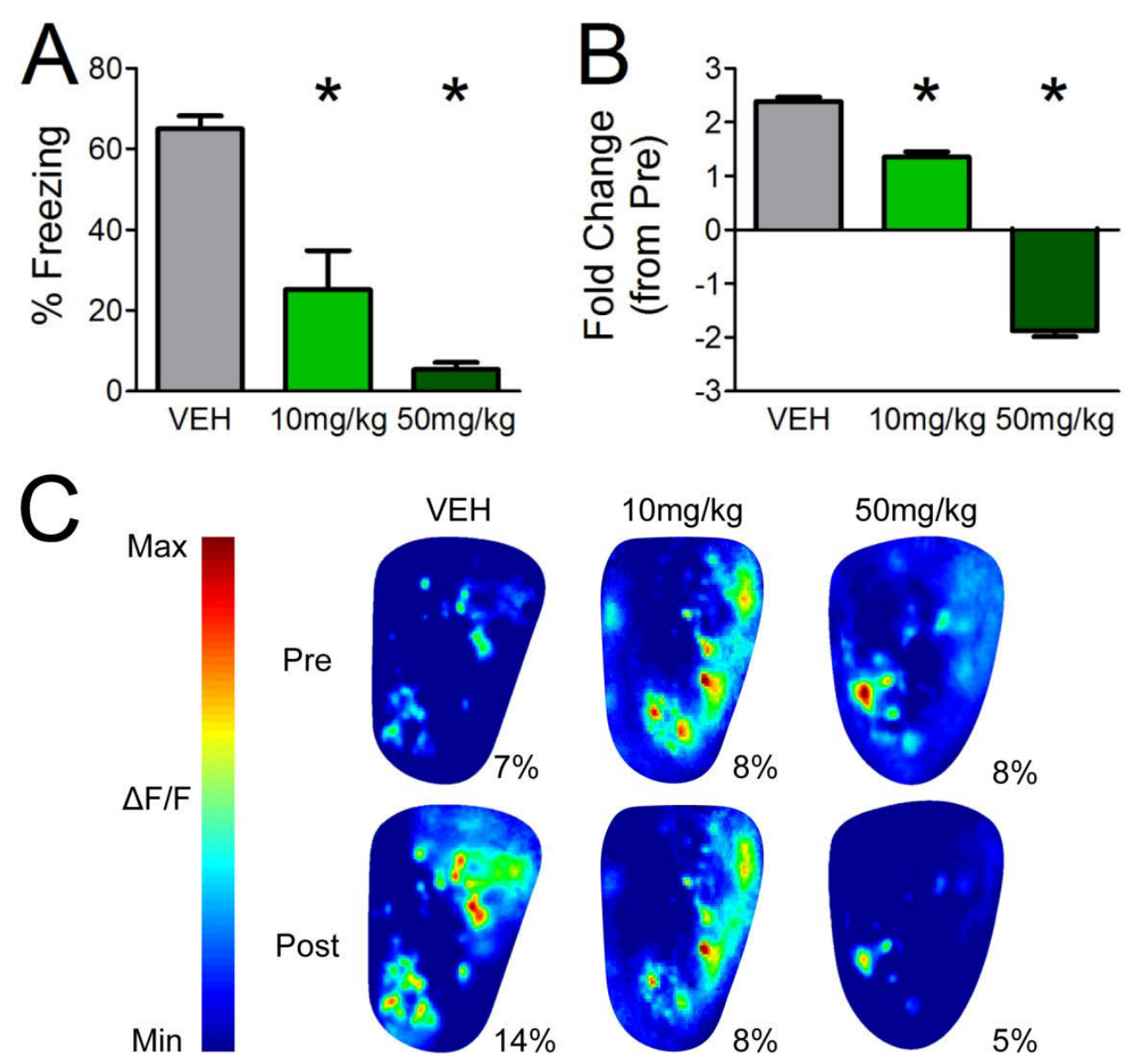

Figure 3-5. Activation of mAChRs are necessary for odor-shock driven OB glomerular enhancements.

Mice were subjected to awake-wide field imaging procedures before and after odor-shock pairing to determine the effect of systemic scopolamine (SCOP) during training on later glomerular responses. (A) When tested for learned fear, ice receiving either $10 \mathrm{mg} / \mathrm{kg}$ or $50 \mathrm{mg} / \mathrm{kg}$ SCOP freeze significantly less to the CS, E5, than mice who received vehicle (VEH) prior to olfactory fear conditioning. (B) The mice that received $10 \mathrm{mg} / \mathrm{kg}$ during fear conditioning exhibit less post-training glomerular enhancements than VEH mice and those that received $50 \mathrm{mg} / \mathrm{kg}$ SCOP displayed suppression of the odor-shock induced enhancements seen in VEH mice. (C) Psuedocolored averaged Pre and Post glomerular response maps from representative $\mathrm{VEH}, 10 \mathrm{mg} / \mathrm{kg} \mathrm{SCOP}$, and $50 \mathrm{mg} / \mathrm{kg}$ SCOP mice where the pseudocolor scale is based on the day with the maximum observed responses (Post for VEH and Pre for $10 \mathrm{mg} / \mathrm{kg}$ and $50 \mathrm{mg} / \mathrm{kg}$ ) to avoid oversaturation of psuedocolored maps. The approximate value of the maximum observed responses $(\Delta \mathrm{F} / \mathrm{F})$ used for pseudocolor scale is listed to the right of each map. The maps demonstrate posttraining glomerular enhancements in VEH mice, minimal change to a slight decrease in $10 \mathrm{mg} / \mathrm{kg}$ mice, and a drastic suppression of glomerular responses in $50 \mathrm{mg} / \mathrm{kg}$ mice. Together, this reveals that systemic antagonism of mAChRs blocks both olfactory fear learning and associated CS-specific glomerular enhancements. Data presented as mean \pm SEM. 
conditioning when learning was blocked by high doses of SCOP. Together this data suggests that muscarinic neurotransmission is required for both olfactory fear learning and CS-specific glomerular enhancements.

\section{Discussion}

Using a combination of in vivo pharmacology, optogenetics, and awake wide-field calcium imaging in conjunction with olfactory fear conditioning, we investigated the role of muscarinic acetylcholine neurotransmission in olfactory fear learning. The results demonstrate that acquisition, but not expression, of olfactory fear learning requires ACh neurotransmission through muscarinic receptors, specifically mAChR1, in the OB. Importantly, direct $\mathrm{OB}$ antagonism of $\mathrm{mAChRs}$ alters olfactory driven behaviors, establishing that the lack of learning is not a consequence of reversible anosmia during olfactory fear conditioning. Furthermore, stimulating release of OB ACh during odor shock pairing appears to increase the strength of fear learning. Finally, we demonstrate that activation of $\mathrm{mAChRs}$ during odor-shock pairing appears to be necessary for $\mathrm{OB}$ plasticity that results in enhanced CS-evoked glomerular responses; however, given systemic administration of mAChR antagonists, we cannot conclude whether this is part of the same mechanism requiring activation of $\mathrm{mAChRs}$ in the OB during acquisition for fear learning to occur. Together, these studies identify a new role for $\mathrm{mAChRs}$ in associative olfactory fear learning; however, the exact mechanism by which mAChR activation during odor-shock pairing allows for acquisition of fear learning remains unknown.

Our findings confirm previous reports that $\mathrm{mAChRs}$ are inextricably linked to olfactory learning. Both genetic (Chan et al., 2017) and pharmacological inhibition of mAChRs suppresses olfactory appetitive and aversive learning (Ravel et al., 1994; Kroon and Carobrez, 2009; Pavesi et al., 2012; Devore et al., 2014; Silva et al., 2015). Our findings expand on these previous reports by demonstrating OB ACh signaling, specifically through $\mathrm{mAChR}$, is required during olfactory fear conditioning; however, the underlying mechanism remains unclear. Several previous reports establish that ACh release into the $\mathrm{OB}$ and subsequent activation of $\mathrm{AChRs}$ can modulate excitability of $\mathrm{OB}$ glomeruli and OB output cells (Chaudhury et al., 2009; Devore et al., 2012; Ma and Luo, 2012; Rothermel et al., 2014; Bendahmane et al., 2016). Furthermore, models including pharmacological blockade of mAChRs in the OB result in altered OB network dynamics, which, in turn, decreases the activation of PCx, cortical plasticity, and learning (Devore et al., 2014). This suggests that mAChRs may regulate olfactory input to PCx during acquisition to subsequently enable olfactory learning. However, it is also possible that the effect blocking $\mathrm{OB}$ mAChRs on $\mathrm{OB}$ output propagates beyond $\mathrm{PCx}$ to other brain regions, such as hippocampus by altering beta oscillations (Kay and Beshel, 2010; Kay, 2014; Osinski et al., 2018). While the exact mechanisms beyond the OB remain unclear, activation of $\mathrm{OB}$ mAChRs in olfactory aversive learning likely regulates OB output, which ultimately leads to olfactory learning. Interestingly, our findings replicate previous reports that $\mathrm{ACh}$ modulation primarily affects acquisition but has little to no effect on 
expression of previous olfactory learning (Saar et al., 2001; Chapuis and Wilson, 2013; Linster and Cleland, 2016).

While ACh modulates several OB cell types including MCs, granule cells (GCs), and periglomerular cells (Nickell and Shipley, 1988; Ravel and Pager, 1990; Castillo et al., 1999; Pressler et al., 2007; Chaudhury et al., 2009), many studies suggest that activation of mAChRs, especially mAChR1, increases excitability of GCs (Pressler et al., 2007; Smith and Araneda, 2010; Smith et al., 2015), which increases synchronization of MC spike timing and oscillatory power (Li and Cleland 2013). This increase in MC synchrony is thought to lead to more synchronous input to PCx and optimized cortical learning. Another possibility is that $\mathrm{OB}$ ACh reduces the inhibitory drive of OB GCs, which could facilitate transmission of olfactory information from OB output neurons to higher processing centers (Elaagouby et al., 1991). Altering inhibitory drive of GCs could also lead to decreased inhibition of neighboring GCs (Castillo et al., 1999) thereby sharpening the receptive fields of $\mathrm{OB}$ output cells. It may also be true that activation of AChRs could inhibit glomerular layer inhibitory interneurons neurons (Crespo et al., 2000; Pignatelli and Belluzzi, 2008; Liu et al., 2015), thereby leading to increased responses of excitatory cells. Future electrophysiological experiments are needed to understand the exact OB circuit level alterations induced by olfactory aversive learning before a full understand of the role ACh plays in such changes can be obtained.

Given olfactory learning is impeded when antagonism of mAChRs is confined to the granule cell layer but not affected when antagonism is confined to the glomerular layer (Ravel et al., 1994), mAChRs most likely modulate the MC/GC circuit, leading to increased MC synchrony and output. Enhanced synchrony and strengthened OB output could explain how optogenetically increasing OB ACh during odor-shock pairing results in strengthened olfactory learning. This idea is in line with previous reports that high ACh facilitates learning by enabling long term potentiation (Linster and Cleland, 2016). However, optogenetic stimulation of OB ACh release cannot resolve whether the facilitated learning is an effect of signaling through nAChRs, mAChRs, or both. Future studies are needed to determine the extent to which these different AChRs and their subtypes contribute to enhancement of olfactory fear learning.

Our findings expand on previous reports by demonstrating $\mathrm{mAChR}$ activation during olfactory learning is required for OB plasticity that results in enhanced glomerular responses, thus providing a mechanism by which $\mathrm{mAChRs}$ enable olfactory learning. However, imaging experiments required the use of systemic SCOP administration and, therefore, cannot determine whether signaling through $\mathrm{mAChRs}$ is required in the $\mathrm{OB}$ for OB plasticity. For example, $\mathrm{PCx}$ cells express $\mathrm{mAChRs}$ so it is possible activation of $\mathrm{PCx}$ $\mathrm{mAChRs}$ are responsible for modulating OB plasticity during learning and that plasticity, but not learning, could still occur in the OB after $\mathrm{mAChR}$ antagonism during odor-shock pairing. While we cannot conclude the direct mechanism by which $\mathrm{mAChRs}$ regulate odor-shock pairing-induced OB plasticity, it is important to note that other methods of blocking learning do not suppress CS-specific glomerular enhancements (Ross and Fletcher, 2018a). Since CS-specific glomerular enhancements remain when learning is blocked, the loss of enhancements demonstrated here cannot be attributed simply to lack 
of learning. Instead, mAChRs must play an integral role in this form of OB plasticity. We also did not determine if the enhanced glomerular responses are dependent on activation of a specific subtype of mAChRs during odor-shock pairing, meaning we cannot be sure that olfactory learning and OB plasticity are both due to activation of $\mathrm{mAChR} 1$ during odor-shock pairing, nor did we investigate the exact means by which mAChRs induce OB plasticity. Future studies can focus on delineating the specific roles of $\mathrm{mAChR}$ subtypes on OB plasticity, possibly through chemogenetic methods.

In summary, this study clarifies the role of ACh in olfactory associative aversive learning. Previous reports using systemic antagonists indicated mAChRs, but not nAChRs, are required during odor-shock pairing for olfactory learning to take place.

However, this did not address whether ACh signaling through mAChRs is required in the $\mathrm{OB}$ or the extent to which olfactory aversive learning is mediated by specific $\mathrm{mAChR}$ subtypes. Using OB infusion of $\mathrm{mAChR}$ antagonists, we determined that activation of mAChRs, specifically the $\mathrm{m} 1$ subtype, is required directly in the OB during odor-shock pairing for acquisition of olfactory fear learning. Additionally, we were able to confirm previous reports that blockade of $\mathrm{mAChRs}$ does not interfere with expression of previously learned olfactory fear. Furthermore, optogenetic stimulation of OB ACh during odor-shock pairing appears to increase strength of olfactory learning. Finally, using systemic pharmacology and awake, wide-field imaging, we demonstrate muscarinic ACh signaling is essential during odor-shock pairing for associative learning-independent CS-specific glomerular enhancements. Together these studies demonstrate the importance of $\mathrm{OB} \mathrm{ACh}$ for olfactory learning and related plasticity. 


\section{CHAPTER 4. OLFACTORY FEAR CONDITIONING INDUCES ROBUST REORGANIZATION OF SALIENCE AND IDENTITY-CODING IN OLFACOTRY BULB OUTPUT CELLS}

\section{Introduction}

During associative fear learning an initially irrelevant stimulus (the conditioned stimulus, CS) is temporally paired with another stimulus that naturally induces a fear response, such as foot shock. As a result of the pairing, the organism learns that the once meaningless CS predicts the fear inducing stimulus and begins to respond to the CS with a fear response even in the absence of the original fear inducing stimulus (Pavlov, 1927). Inexplicably, the learned fear often extends beyond the CS, which was paired with a fear inducing stimulus, to completely neutral, unlearned stimuli, a process known as stimulus generalization (Pavlov, 1927; Pavesi et al., 2012; Dunsmoor and Paz, 2015; Resnik and Paz, 2015; Jasnow et al., 2017). One theory of fear generalization is that it arises from "cognitive processes", in which organisms adopt a better-safe-than-sorry strategy (Shepard, 1987; Herry and Johansen, 2014; Dunsmoor and Paz, 2015). In this theory, organisms respond to neutral stimuli as if they were the CS, despite perceptual systems coding them as distinct from the CS. Another theory suggests fear generalization results from a failure to discriminate (Lashley and Wade, 1946; Ghosh and Chattarji, 2015; Zaman et al., 2019). This theory posits that the initial learning alters sensory coding in a manner that renders organisms incapable of perceptually distinguishing neutral stimuli from the CS. While fear generalization is common amongst several species, including humans, the underlying cause, better-safe-than-sorry or failure of perceptual discrimination, remains unknown.

The mouse olfactory system is an excellent model for studying underlying mechanisms of fear generalization. Each odor is uniquely coded in the main olfactory bulb (OB) in neuropil structures called glomeruli, which are the sites of synaptic contact between peripheral olfactory sensory neuron (OSN) axons and OB output cells. Odor molecules bind to OSNs and information regarding odor identity is transmitted to specific glomeruli in a spatiotemporal pattern based on the molecular features of the bound odorant (Buck and Axel, 1991; Shipley and Ennis, 1996) that conveys odor identity (Wachowiak and Cohen, 2001; Spors and Grinvald, 2002; Bozza et al., 2004; Mori et al., 2006; Fletcher et al., 2009; Storace and Cohen, 2017). Recent research suggests initial olfactory coding is not invariant and can be altered through experience (Freeman and Schneider, 1982; Di Prisco and Freeman, 1985; Friedrich and Stopfer, 2001; Fletcher and Wilson, 2003; Kato et al., 2012; Li et al., 2015; Chu et al., 2016; Geramita and Urban, 2016; Yamada et al., 2017; Ogg et al., 2018); therefore, examining fear learning-induced changes in olfactory coding may shed light on mechanism of fear generalization.

Olfactory fear conditioning enhances the neural activity of several cell populations in the adult OB (Freeman and Schneider, 1982; Fletcher, 2012; Kass et al., 2013; Kass and McGann, 2017), even following inhibition of neural fear centers and appears to increase the representational similarity between neutral odors and CS (Ross 
and Fletcher, 2018b). This is in line with studies in other sensory systems. Auditory fear conditioning shifts and broadens the receptive fields of auditory cortex neurons (Bakin and Weinberger, 1990; Scheich and Zuschratter, 1995; Bakin et al., 1996; Weinberger and Bakin, 1998; Weinberger, 2004). Furthermore, whisker deflection, when paired with aversive stimuli, causes expansion of whisker somatotopic representation in barrel and somatosensory cortices (Siucinska and Kossut, 1996). Together, this demonstrates that fear conditioning alters sensory processing in primary sensory regions in manners that shift how sensory stimuli are represented and therefore supports the failure of perceptual discrimination hypothesis of fear generalization. However, studies of coding mechanisms underlying fear generalization are tenuous and the extent to which cells in primary sensory regions tasked with transmitting information regarding stimulus identity are modified remains unclear.

Consequently, we use awake, 2-photon calcium imaging (2P) in mice to investigate fear-learning induced changes in identified superficial tufted cells (STCs) and mitral cells (MCs), which are the output cells of the OB. By recording odor-evoked responses in each of these cell types to the CS as well as other odorants to which fear is generalized, we demonstrate olfactory fear learning strengthens odor-evoked responses in output cells. In contrast, mice in control conditions exhibit weakened odor-evoked responses in both cell types without modifying basic identity coding. Furthermore, we establish that, while STC responses are enhanced following odor-shock pairing, the similarity of odor-evoked responses between the CS and neutral odors remains unchanged, indicating STCs may convey information regarding odor salience rather than identity. After fear learning, MCs display stronger odor-evoked responses primarily to neutral odors and the overall effect appears to increase the similarity of responses between these odors and the conditioned stimulus, suggesting MCs likely code odor identity and that the observed changes contribute to failure of perceptual discrimination. Together, these results represent that fear conditioning transforms early sensory coding and likely provide and initial basis for fear generalization by enhancing both salience and similarity of incoming sensory information.

\section{Methods and Materials}

\section{Animals}

Experiments were carried out using adult (8-16 weeks) male and female C57BL/6J-Tg(Thy1-GCaMP6f)GP5.11Dkim/J (Jax Stock No: 024339), C57BL/6JTg(Thy1-GCaMP6s)GP4.12Dkim/J (Jax Stock NoL 025776), or crosses of FVB/NTg(Thy1-cre)1Vln/J (Jax Stock No: 006143) with B6J.Cg-Gt(ROSA)26Sor ${ }^{\text {tm95.1(CAG- }}$ GCaMP6f)Hze/MwarJ (Jax Stock No: 028865). In all cases, the resulting mice express the fluorescent calcium indicator GCaMP6s or GCaMP6f under the Thy1 promoter. In some cases, crosses between B6;CBA-Tg(Tbx21-cre)1Dlc/J (Jax Stock No: 024507) and

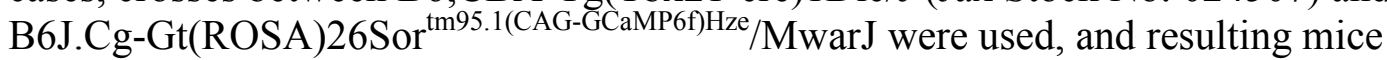
express GGCaMP6f under the T-box 21 promoter. Each of these crosses allows 
visualization of OB neurons, such as juxtaglomerular cells (JGCs), STCs, and MCs. Exact cell types recorded were determined as described in Quantification and Statistical Analysis. We observed no differences in neural responses between generated strains. All experimental protocols were approved by the University of Tennessee Institutional Animal Care and Use Committee.

\section{General Methodology}

\section{Surgical procedures}

Mice were placed on antibiotic food (Uniprim) for 7 days prior to surgery and maintained on it for an additional 7 days following surgery. Prior to the surgery, mice were given a cocktail of ketamine/xylazine $(100 / 10 \mathrm{mg} / \mathrm{kg}$ i.p.) to induce anesthesia and a cocktail of carprofen/dexamethasone $(5 / 0.2 \mathrm{mg} / \mathrm{kg}$ s.c.) as an analgesic and antiinflammatory agent. Mice were then secured in a stereotaxic apparatus (NARASHIGE Group) and a $3 \mathrm{~mm}$ diameter of skull overlying both olfactory bulbs was removed and then fitted with a $3 \mathrm{~mm}$ diameter cover glass (Figure 4-1A). The cover glass was secured to the area with superglue and dental cement and then filled with silicone. Once dry, the skin was sutured closed. Approximately one week after the cranial window surgery, mice were once again anesthetized with a cocktail of ketamine/xylazine and given carprofen/dexamethasone before a stainless steel head bar was attached to the skull. The head bar was secured with an anchor screw set in the parietal bone. The entire exposed skull was then covered with superglue and dental cement. Mice were given at least 24 hours to recover from the minor head bar implantation surgery.

\section{Odorants}

A subset of odorants (Sigma-Aldrich) from a previously established odorant panel was used (Ross and Fletcher, 2018a). The panel consisted of ethyl valerate (E5), ethyl butyrate (E4), ethyl hexanoate (E6), and benzaldehyde (BZ). E5 was used as the CS. The other odorants were used for their perceptual and representational similarity or dissimilarity to E5 to assess responses to chemically diverse odors. E4 and E6 represent structurally similar odorants whereas BZ, an aldehyde, represents a structurally dissimilar odorant.

\section{2-photon imaging}

Imaging was performed with a Zeiss 7MP 2P microscope equipped with a Zeiss $20 \mathrm{x}$ objective. Imaging trials were collected at 512 x 512 resolution at $\sim 2.5-3.25 \mathrm{~Hz}$ with imaging regions of $300-450 \mu \mathrm{m}$. Awake mice were first head-fixed to a head holding device (NARISHIGE Group) via the stainless steel head bar affixed to their skulls (Figure 4-1B). Mice were exposed to head fixation and 2P for three consecutive days. The first day served as an acclimation day where the quality of the cranial window and optimal depth of imaging were assessed but no data was taken for analysis. The following two days serve as the "Pre" and "Post" imaging time points for analysis, in 

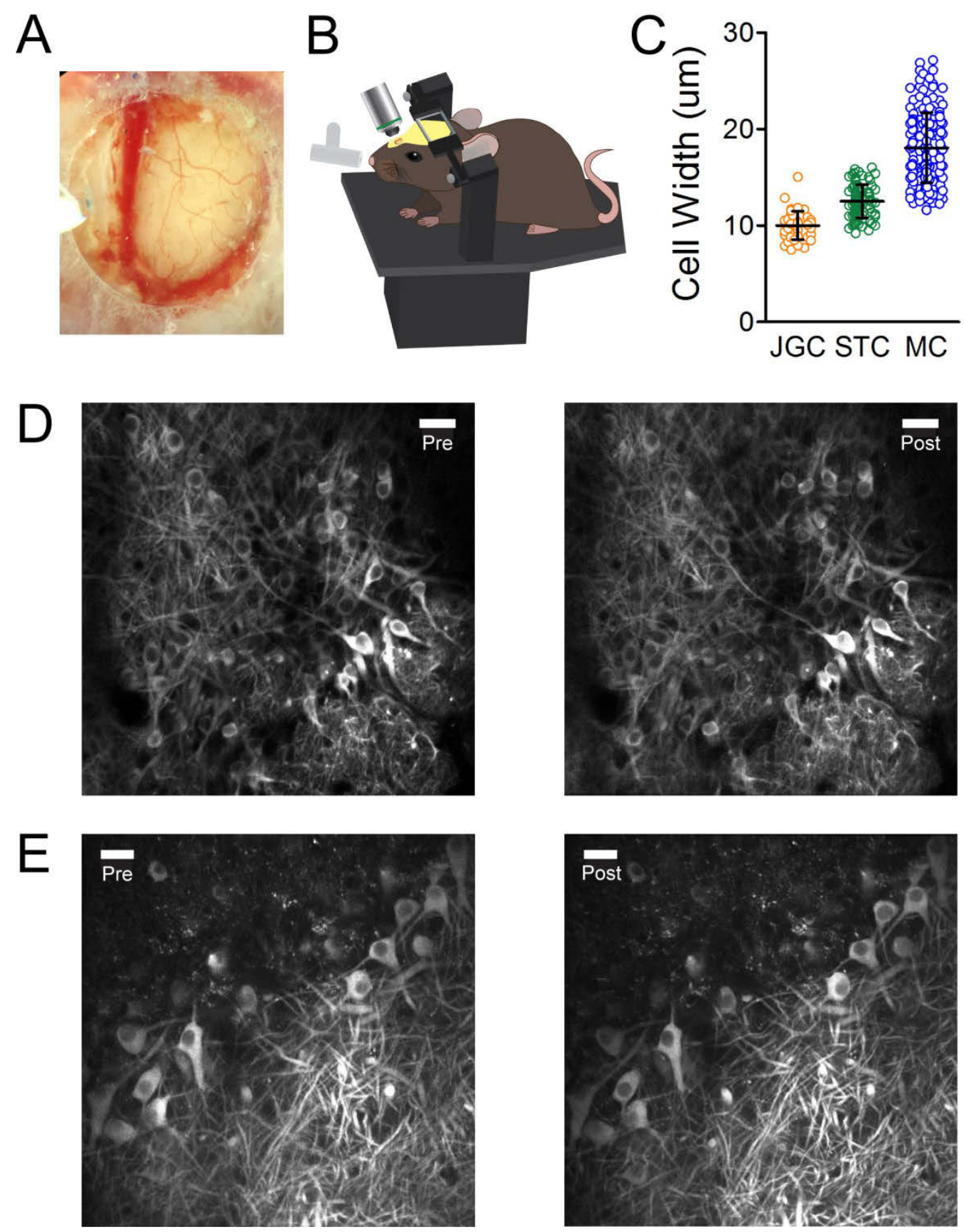

Figure 4-1. Multi-day imaging of single cells in olfactory bulbs of awake mice. (A) Example 2P chronic window. (B) Diagram of head-fixation setup for awake 2P imaging. (C) Distribution of somatic widths measured from imaging mice demonstrating anatomical size difference between JGCs, STCs, and MCs. (D-E) Example field-of-view during 2P imaging of GCaMP6 labeled STCs (D) and MCs (E) before (left) and after (right) conditioning, demonstrating feasibility of tracking the same cells across days. Scale bar $=25 \mu \mathrm{m}$. 
which the same set of cells were recorded for their responses to the panel of odorants described above. Imaging trials consisted of a $5 \mathrm{~s}$ delay before a $2 \mathrm{~s}$ odor presentation for a total of $\sim 40-50$ frames of collected data. A resting z-stack of the imaging region was taken for each animal to aid in cell classification. Odors were presented to mice via a custom made olfactometer at $0.7 \mathrm{~L} / \mathrm{min}$ with a concentration of $0.5 \%$ saturated vapor, which was achieved by flow dilution of odorized air into humidified clean air. Mice were presented each odor at least 4 times each day. Approximately 2 hours following the Pre imaging session, mice were subjected to olfactory fear conditioning as detailed below. Approximately 24 hours later, mice were tested for behavioral freezing (see below) before undergoing the Post imaging session.

\section{Olfactory Fear Conditioning and Testing}

Olfactory fear conditioning and testing was carried out as previously described (Ross and Fletcher, 2018a; c). Briefly, after the Pre imaging session, mice were separated into two groups: Paired, in which they received six E5 presentations co-terminating with foot shock (10s 200ppm E5; 0.8mA, 0.5s shock). Mice in the Control condition were a mixture of unpaired, in which they also received six E5 presentations and 6 foot shocks in the same training session but in an unpaired fashion, or odor only, in which mice received only six E5 presentations and no foot shocks. Approximately 24 hours after conditioning, mice were placed in a novel context located inside an isolation chamber for testing. Mice were allowed several minutes to acclimate to the testing chamber prior to the start of the testing protocol. Each mouse was assessed for their behavioral freezing, a widely used measure of learned fear, for the CS (E5) as well as the other odors used during imaging experiments which allowed testing of breadth of generalization. Testing consisted of an initial 30s absent of any odors followed by one 20s presentation of each odor, spaced 180 s apart. The first 60 s of the testing session in which there was no odor was used as a baseline measure of freezing for comparison to E5 to confirm learning. All testing odors were intensity matched and diluted in mineral oil to achieve an approximate headspace concentration of $200 \mathrm{ppm}$.

\section{Quantification and Statistical Analysis}

All imaging trials were first motion corrected using open-source NoRMCorre code in MATLAB (Pnevmatikakis and Giovannucci, 2017). Fluorescent traces for each cell were then extracted offline using ImageJ. The individual cells were identified in resting fluorescence images and confirmed in z-projections through no air imaging trials before the ROIs were manually drawn around all cells within the imaging region. This was completed independently for the Pre and Post imaging windows and traces were only collected for cells present in both (Figure 4-1D and 4-1E). Cells were then classified based on imaging depth and cell body diameter/morphology. Cells in the mitral cell layer (recordings $\sim 250-300 \mu \mathrm{m}$ from brain surface) were classified as MCs. In order to classify cells in the glomerular layer and/or external plexiform layer we first measured somatic width of each cell (Figure 4-1C) and used resting images and z-stacks to determine 
whether cells had lateral dendrites (Nagayama et al., 2014). If lateral dendrites were present, the cell was designated STC. All others were classified as JGCs and were not used for further analysis as more specific cellular identity was indeterminate. Cell diameters of MCs were also measured. Statistical testing determined a significant difference between cell widths of JGCs, STCs, and MCs $\left(\mathrm{F}_{2,298}=172.1, \mathrm{p}<0.0001, \eta^{2}=\right.$ 0.5453 ), with the average [ $95 \%$ confidence interval] diameter being $10.02 \mu \mathrm{m}[9.55$, $10.49], 12.51 \mu \mathrm{m}[12.13,12.89]$, and $18.07 \mu \mathrm{m}[17.51,18.62]$, respectively.

The trace for each cell was transformed into $\Delta \mathrm{F} / \mathrm{F}\left(\frac{\Delta \mathrm{F}}{\mathrm{F}}=\frac{F_{x}-F_{1}}{F_{0}} \times 100\right)$ by subtracting each data point $\left(\mathrm{F}_{\mathrm{x}}\right)$ by the average pre-odor fluorescence $\left(\mathrm{F}_{1} ; 5\right.$ frames preceding odor onset) divided by the average resting fluorescence $\left(\mathrm{F}_{0} ; 10\right.$ frames preceding odor onset). The $\Delta \mathrm{F} / \mathrm{F}$ values were then smoothed by applying a rolling average $(\mathrm{n}=3)$. The smoothed $\Delta \mathrm{F} / \mathrm{F}$ traces were averaged for each odor to obtain an average daily odor response for each odor. Once average daily odor responses were obtained for each animal, all traces were separated by cell type and aligned by onset of odor response. For initial analysis, the odor-evoked response for every cell was obtained by averaging the $\Delta \mathrm{F} / \mathrm{F}$ values during the odor on window (10 frames). A fold change value was then calculated to determine the change in response post-training compared to pre-training. Some cells change polarity of response (from negative to positive or vice versa). It is not possible to calculate true fold change in such values; therefore, the opposing polarity was set to 1 or -1 , allowing for an approximate fold change to be obtained. Fold change values falling between -1 and 1 indicate no change in the odorevoked response.

Odor responses for STCs occurred primarily during the odor-on window, while several MCs had their first response after odor offset. MCs responses were clustered using a k-means cluster analysis with five defined clusters after being normalized to fit traces, beginning at odor onset and persisting for a total of 30 frames, between -1 and 1 (Figure 4-2A and 4-2C). An additional k-means cluster of odor-evoked MC responses with 2 defined clusters appeared to separate $\mathrm{MC}$ responses based on whether the overall evoked response was excitatory (positive normalized values) or inhibitory (negative normalized values) regardless of whether the excitatory/inhibitory response occurred during the odor or after (Figure 4-2B and 4-2D). Therefore for initial analysis, MCs were categorized as excitatory (E) or inhibitory (I). Cells were classified as non-responsive only if the average of a sliding 10 frame window starting with odor-onset and ending at the end of the imaging trial failed to achieve $\pm 2 \mathrm{RMS}$ (root mean squared) of the average pre-odor fluorescence.

Temporal correlations of odor responses were performed in each cell type for each animal by obtaining a correlation value for the population response between E5 and each other odor at each frame. This was completed separately each day to obtain the pretraining correlation between E5 and other odors and the post-training correlation between E5 and other odors. Temporal correlations were then averaged together for mice belonging to the same training condition. For statistical analysis, temporal correlation 

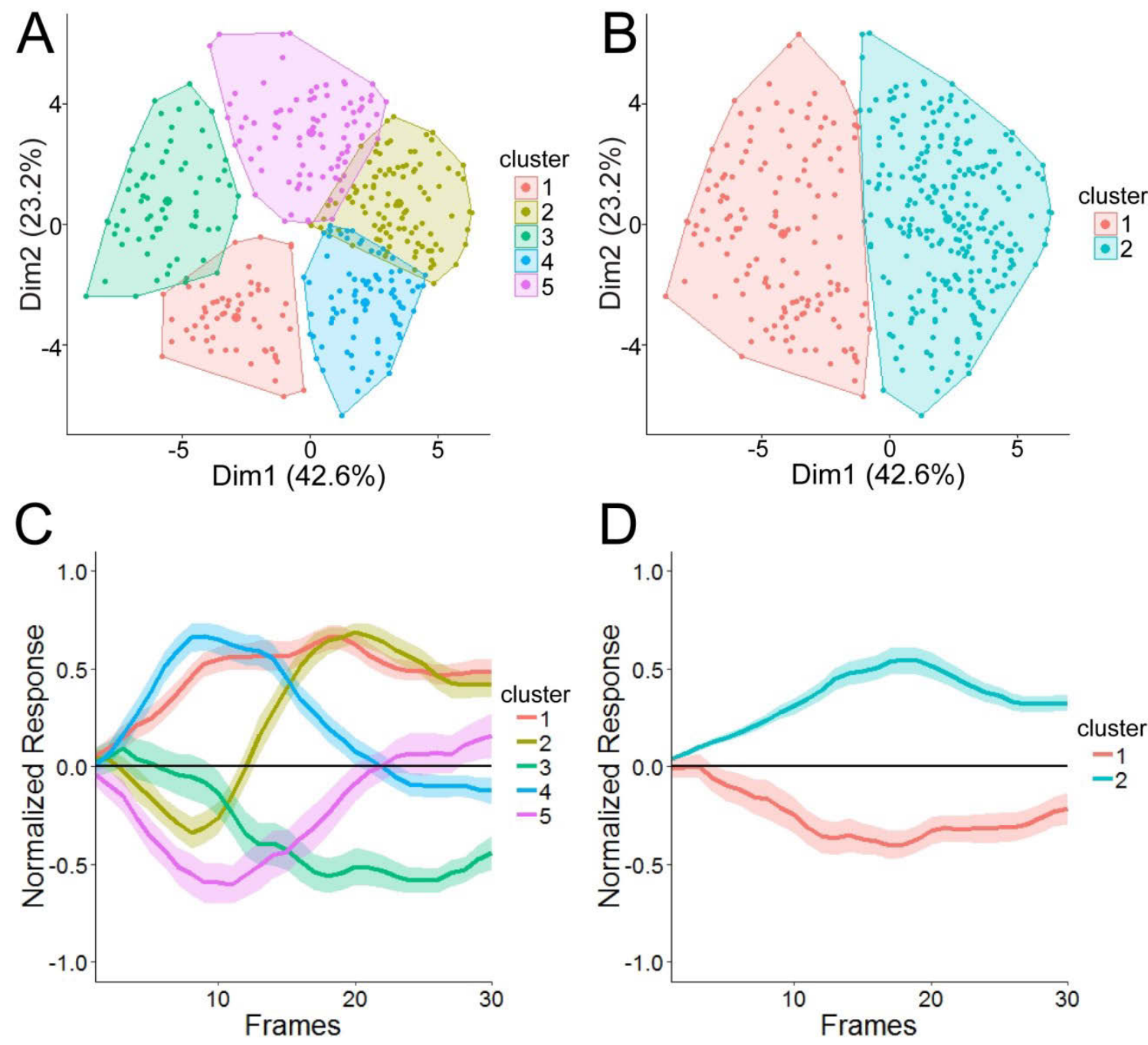

Figure 4-2. Mitral cells exhibit varied response profiles.

(A-B) Cluster analysis (k-means) of mitral cells based on odor-evoked responses beginning with odor onset and ending with trial cessation. MCs can be grouped into five defined clusters (A) and two defined clusters (B). (C-D) Averaged cluster response based on five (C) and two (D) defined clusters. All clustered responses, regardless of whether they are defined by five or two clusters, demonstrate either excitatory or inhibitory odorevoked responses during the odor-on phase of the imaging trial (frames 1-10). Data displayed as mean \pm 2 SEM. 
values were then averaged into bins representing the average pre-odor onset correlation, the average odor-on correlation, and the average post-odor correlation.

For quantification of learned fear, behavioral freezing was measured from the onset of each stimulus presentation for a total of 60 seconds and is reported as $\%$ of the 60 s bin spent freezing.

Statistical tests were analyzed using R statistical analysis software, Prism software (GraphPad, version 5.03), or IBM SPSS 22.0. Equal variances were tested, and suitable corrections were made when necessary. A one-way ANOVA was used to assess behavioral freezing and a Dunnett's t post hoc test was performed where appropriate with freezing to E5 as the comparison point. Two-tailed paired samples t-tests were used to compare raw odor-evoked STC and MC population responses before and after training for individual groups and were used to analyze binned temporal correlation data. Wilcoxon Signed Rank tests were used to compare fold-change data to the hypothetical 0 fold change, and the standardized test statistic $\left(\mathrm{W}^{*}\right)$ is reported 2-sample Wilcoxon Signed Rank tests were used to compare fold-change data across specific odors (W).

\section{Results}

\section{Olfactory Aversive Conditioning Induces Behavioral Generalization Across Structurally Similar Odorants}

Following the Pre Imaging session, all mice were split into either a Control or Paired training condition. Approximately 24 hours after training, all mice were tested for their behavioral freezing to the CS, a widely used measure of learned fear, and to the chemically similar odors E4 and E6 (Figure 4-3). Additional mice not subjected to imaging were subjected to training and testing ( $\mathrm{n}=3$ Control and 1 Paired) and are included in behavioral analysis. No behavioral differences were noted between imaging and non-imaging mice. Mice in the Control condition $(n=6)$ did not exhibit more freezing behaviors during odor presentations than they did in the baseline minute $\left(\mathrm{F}_{3,20}=\right.$ $\left.0.7248, p=0.5490, \eta^{2}=0.0981\right)$, indicating they did not learn to fear odors. In contrast, freezing behavior reveals mice in the Paired condition $(n=8)$ learned to fear odors $\left(\mathrm{F}_{3,28}\right.$ $\left.=13.17, \mathrm{p}<0.0001, \eta^{2}=0.5853\right)$. Paired mice froze significantly more in the minute during the E5 presentation than they did in the baseline minute $(\mathrm{p}<0.0001)$ and there was no significant difference between freezing to the CS and the neutral odors E4 ( $\mathrm{p}=$ $0.767)$ or E6 $(p=0.877)$. Freezing to the neutral odors in a similar manner as the CS is indicative of behavioral fear generalization. 

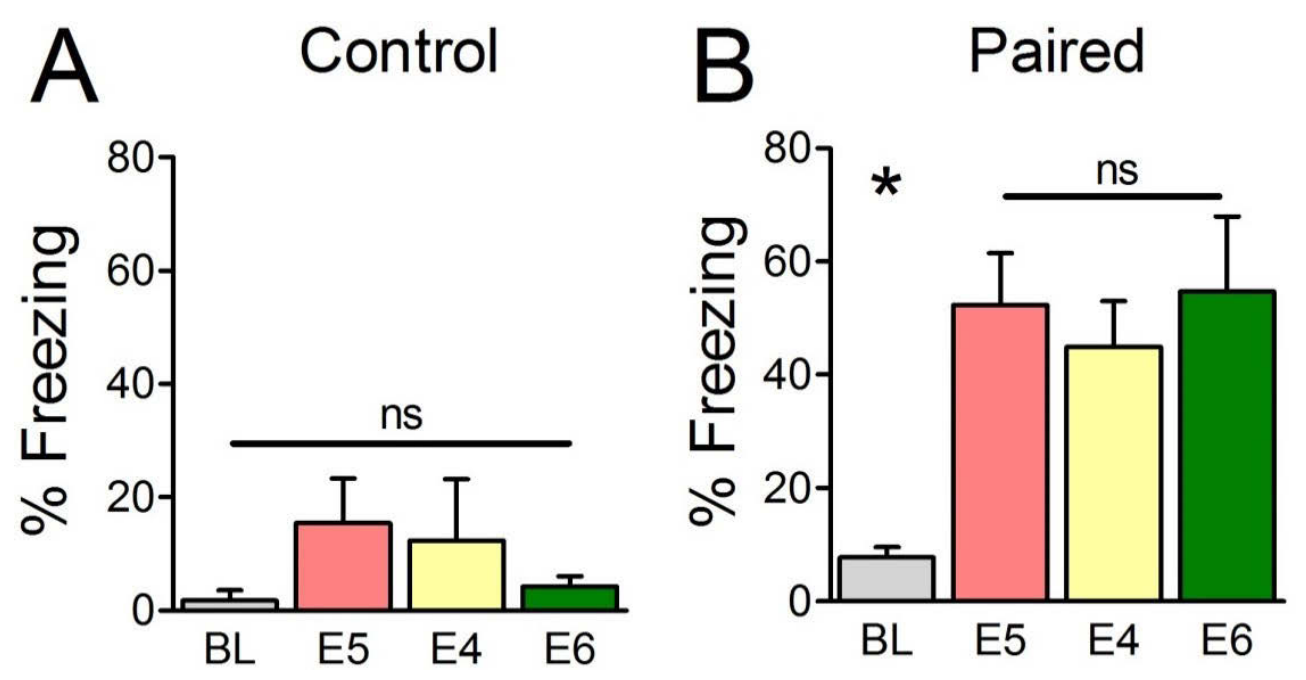

Figure 4-3. Fear conditioning leads to behavioral fear generalization.

(A) Control mice do not exhibit freezing behaviors to odor presentations different from baseline (BL) freezing when tested $\sim 24 \mathrm{~h}$ following control conditioning. (B) Mice receiving paired presentations of $\mathrm{E} 5$ and foot shock freeze significantly more to presentations of E5 than BL and do not exhibit differential freezing to neutral odors, signifying generalization of behavioral fear. Data displayed as mean $\pm \mathrm{SEM},{ }^{*} \mathrm{p}<0.05$. 


\section{Olfactory Aversive Conditioning Enhances Odor-Evoked Responses in Superficial Tufted Cells}

After behavioral testing, mice underwent the Post imaging session to determine the effects of olfactory fear learning on odor-evoked responses in STCs, a population of OB output cells. STCs typically have strong odor-locked responses with little to no inhibitory responses (Nagayama et al., 2004); therefore, STCs were analyzed as a single population. We first tested whether raw odor-evoked responses were altered during the odor-on window by conditioning experience. Odor-evoked STC responses of Control mice were significantly weaker after control conditioning (Figure 4-4A and 4-4B; $\mathrm{t}_{122}=$ $6.212, \mathrm{p}<0.0001$; Mean Pre $=31.39 \pm 3.213$, Mean Post $=20.27 \pm 2.779)$. Of the 123 cell-odor pairs, $14.63 \%$ were deemed unresponsive. Of the remaining cell-odor pairs, $18.095 \%$ (15.45\% of all cell-odor pairs) were stronger after training whereas $81.905 \%$ (69.92\% of all cell-odor pairs) were weaker. This ratio was relatively conserved across specific odor-evoked responses (Figure 4-4C). In contrast, odor-evoked STC responses of Paired mice were significantly stronger after odor-shock pairing and learning

(Figure 4-4D and 4-4E; $\mathrm{t}_{255}=4.241, \mathrm{p}<0.0001$; Mean Pre $=62.18 \pm 4.120$, Mean Post $=$ $72.93 \pm 4.328)$. Of the 256 cell-odor pairs, $25.39 \%$ were deemed unresponsive. Of the remaining cell-odor pairs, $82.199 \%$ (61.33\% of all cell-odor pairs) were stronger and only $17.801 \%$ (13.28\% of all cell-odor pairs) were weaker after olfactory fear learning, which did not significantly differ across specific odor-evoked responses (Figure 4-4F). This suggests most STC odor responses are stronger after odor-shock pairing regardless of which odor evokes the STC response.

Raw responses vary significantly across cell-odor pairs (from 0 to $\sim 400 \% \Delta \mathrm{F} / \mathrm{F}$ ), which can greatly skew results when averaged; therefore, responses were transformed into fold change (from Pre). Responses were also sorted into odor to determine whether responses evoked by specific odors change differently after training. Control mice exhibit significantly weaker STC responses (Figure 4-4G) to presentations of E5 (W* $=-2.286$, $\mathrm{p}=0.0231)$, and $\mathrm{E} 4\left(\mathrm{~W}^{*}=-2.597, \mathrm{p}=0.0098\right)$ but not $\mathrm{E} 6\left(\mathrm{~W}^{*}=0.551, \mathrm{p}=0.582\right)$, while paired mice exhibit significantly stronger STC responses (Figure 4-4H) to all odors (W* $=2.536,4.304,2.293$ and $\mathrm{p}=0.0113,<0.0001$, and 0.0221 for E5, E4, and E6, respectively). Many of the STCs respond to the neutral odors E4 and E6 as well as the conditioned odor E5. While the population of cell-odor pairs for each odor were significantly stronger after fear conditioning, a small population of STCs from Paired mice exhibit weaker responses after olfactory fear conditioning (Figure 4-4F).

Therefore, we next investigated whether STCs that respond to neutral odors and E5 exhibit different learning-induced changes than those that respond only to neutral odors (Figure 4-4I). Cells from mice in the Paired condition that respond to E4 as well as E5 exhibit significantly stronger responses after training $\left(\mathrm{W}^{*}=3.637, \mathrm{p}<0.0001\right)$. Cells that respond to E6 as well as E5 are also significantly stronger after training $\left(\mathrm{W}^{*}=3.735\right.$, $\mathrm{p}<0.0001)$. Interestingly, cells that respond to E4 but not E5 are also significantly stronger after training $\left(\mathrm{W}^{*}=2.746, \mathrm{p}=0.006\right)$ but those that respond to E6 but not E5 are $\operatorname{not}\left(\mathrm{W}^{*}=1.633, \mathrm{p}=0.102\right)$. There is no difference when directly comparing the fold change of STCs that respond to E4 and E5 relative those that respond to E4 but not E5 
Figure 4-4. Olfactory fear learning globally increases salience coding in olfactory bulb superficial tufted cells.

(A) Heat maps of the activity of all defined STC-odor pairs in awake Control mice before (left) and after (right) training to E5, E4, and E6. Individual cells are organized in rows and are matched between Pre and Post. White vertical lines demonstrate the odor-on window. (B) Quantification of odor-evoked fluorescence changes in Control mice during the odor-on window for all odors before and after conditioning. (C) Total percentage of Control STCs deemed either non-responsive after training as well as those deemed to have stronger or weaker responses after training than before. On average, Control mice demonstrate decreased odor-evoked responses in STCs (B) and this effect is represented in the majority of recorded cells (C). (D) Heat maps of the activity of all defined STCodor pairs in awake Paired mice before (left) and after (right) training to E5, E4, and E6. In contrast to Control mice, Paired mice demonstrate increased odor-evoked responses in STCs (E) in the majority of recorded cells (F). (G-H) Fold-change of individual odorevoked responses from Pre in Control $(\mathrm{G})$ and Paired $(\mathrm{H})$ mice, demonstrating a significant $\sim 2$-fold weaker response in Control STCs and $\sim 3$-fold stronger response in Paired STCs. (I) Responses in STCs of Paired mice appear significantly stronger after olfactory fear conditioning regardless of whether the STC also responds to the CS; though STCs that respond to the CS might be enhanced more than those that do not. Data displayed as mean $\pm \mathrm{SEM}, * \mathrm{p}<0.05$. 


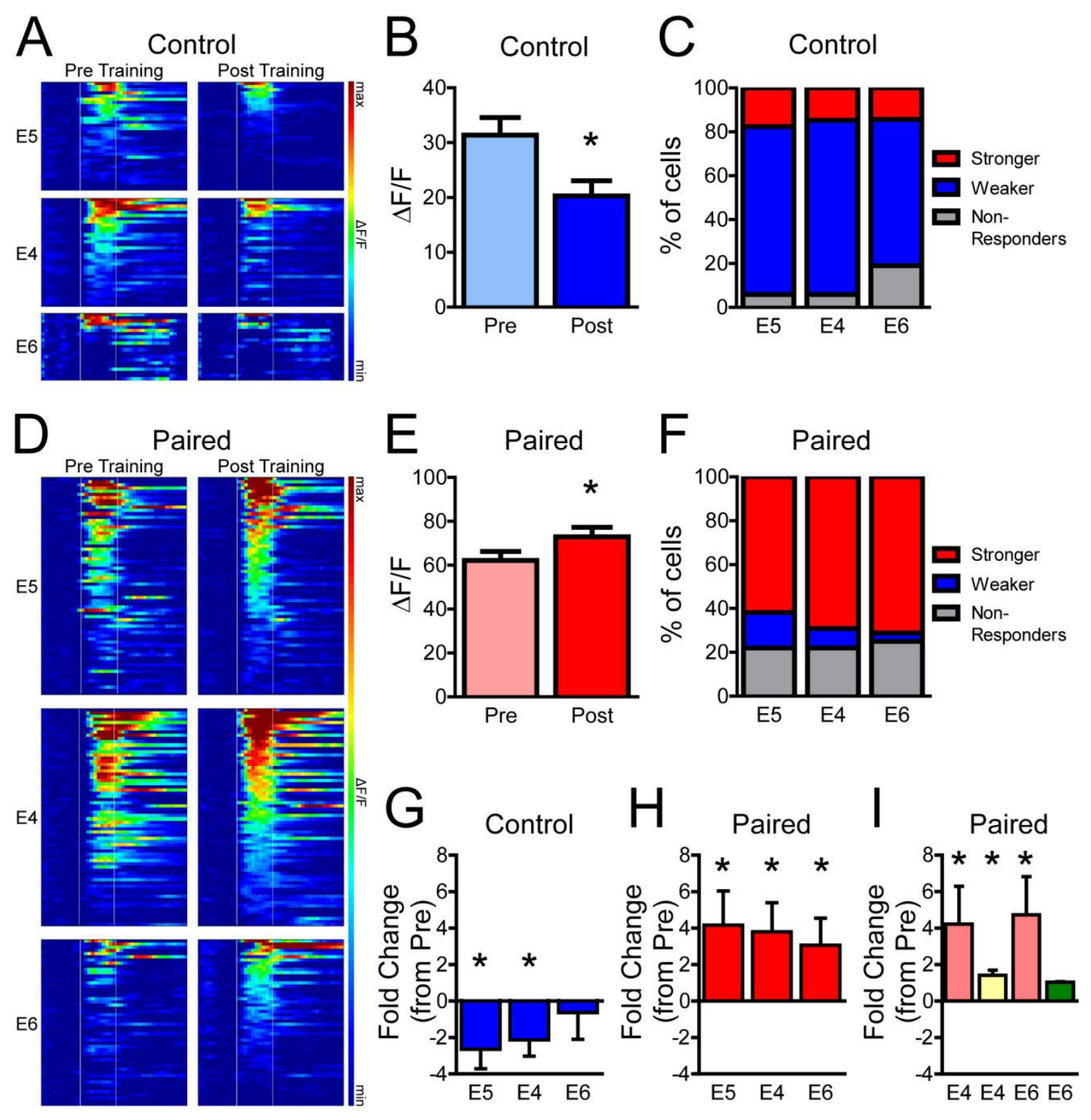


$(\mathrm{W}=2.975, \mathrm{p}=0.085)$. There is also no difference between the learning-induced fold change of STCs that respond to E6 and E5 relative to those that respond to E6 but not E5 $(\mathrm{W}=0.797, \mathrm{p}=0.372)$. While there is no significant difference in neutral odor-evoked fold-change based on whether STCs respond to neutral odors as well as the CS, that may be due largely to the fact there are very few neutral odor responsive cells that do not also respond to the CS $(\mathrm{n}=15)$. By consolidating E4 and E6 STCs that also respond to E5 into a single "Shared" category and STCs that respond to E4 and E6 but not E5 into a single "Unshared" category, the trend toward significance increases $(\mathrm{W}=3.341, \mathrm{p}=$ 0.068 ), suggesting that recording from additional "Unshared" STCs may tease apart learning-evoked differences between the two populations. If true, this suggests fear learning may enhance STCs in a specific manner, based on whether those cells also respond to the CS.

\section{Enhanced Superficial Tufted Cells Responses Do No Alter Coding of Odor Identity}

We next investigated whether the experience-dependent effects on STC responses serve to alter how similarly STCs respond to the CS and neutral odors. Therefore, we measured how similar the population responses to neutral odors were to the E5 response both before and after conditioning experiences (Figure 4-5). There was no significant difference in Pre vs Post conditioning population correlations before $\left(\mathrm{t}_{4}=0.945, \mathrm{p}=\right.$ $0.398)$, during $\left(t_{4}=0.498, p=0.645\right)$, or after $\left(t_{4}=0.409, p=0.703\right)$ odor presentations in Control mice (Figure 4-5A and 4-5C). There was also no significant difference in the Pre vs Post conditioning population correlations before $\left(\mathrm{t}_{10}=0.785, \mathrm{p}=0.451\right)$, during $\left(\mathrm{t}_{10}=-1.201, \mathrm{p}=0.258\right)$, or after $\left(\mathrm{t}_{10}=-1.891, \mathrm{p}=0.088\right)$ odor presentations in Paired mice (Figure 4-5B and 4-5C), suggesting enhanced responses do not make the STC responses to neutral odors more similar to the responses evoked by presentations of the CS.

\section{Mitral Cell Odor Responses Are Enhanced Following Olfactory Aversive Conditioning}

In separate mice, we examined the effects of olfactory fear learning on odorevoked MC responses (Figure 4-6A and 4-6D), the second population of OB output cells. Of the 102 Control MC-odor pairs, $13.73 \%$ were deemed unresponsive. Of the remaining cell-odor pairs, $18.182 \%$ (15.69\% of all cell-odor pairs) were stronger during the odor window after training whereas $81.812 \%$ ( $70.59 \%$ of all cell-odor pairs) were weaker. Similar rates of change are found across all odors (Figure 4-6C). Of the 204 Paired MC-odor pairs, $25.49 \%$ were deemed unresponsive. Of the remaining MC-odor pairs, $80.537 \%$ (58.82\% of all cell-odor pairs) were stronger and only $21.477 \%(15.69 \%$ of all cell-odor pairs) were weaker during the odor window after olfactory fear learning. This is conserved across odors (Figure 4-6F), suggesting odor-shock pairing strengthens most MCs odor-evoked responses independent of odor. 

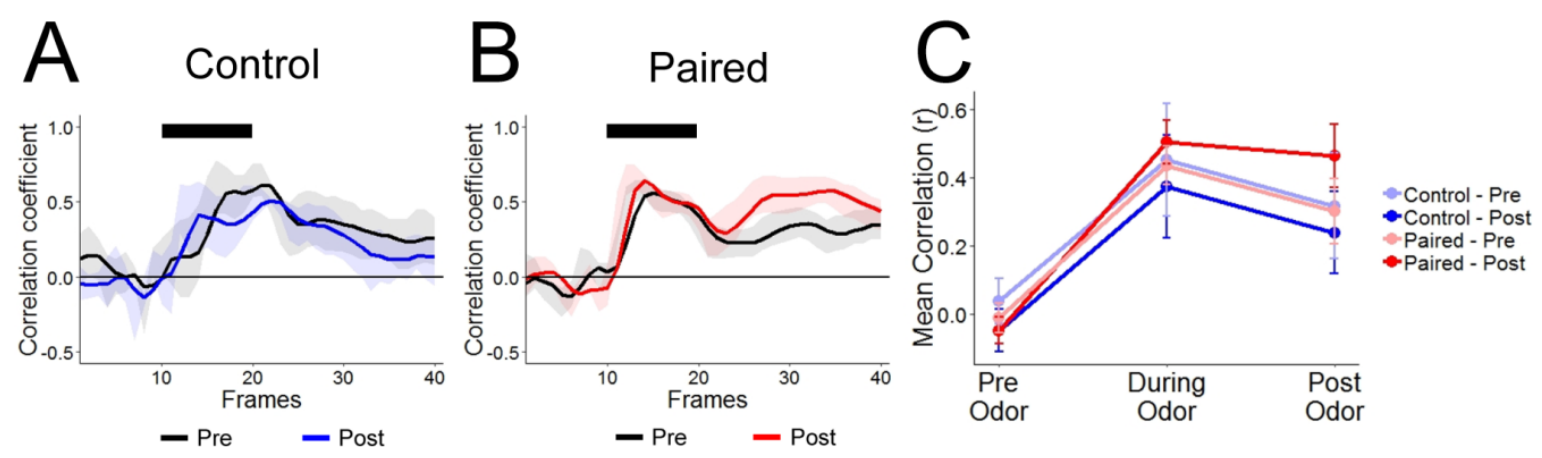

Figure 4-5. Fear learning does not alter odor identity coding in superficial tufted cells.

(A-B) Traces of STC ensemble correlations between the CS and neutral odor responses in Control (A) and Paired (B) mice Pre (black) and Post (blue and red, respectively) conditioning. The correlation coefficients were calculated for the same STC population between pairs of CS-neutral odors at each time point and averaged. Black line indicates odor-presentation. (C) Quantification of average ensemble correlations between the CS and neutral odors for the $2 \mathrm{~s}$ before odor onset (Pre Odor) during the $2 \mathrm{~s}$ odor presentation (During Odor) and for the $4 \mathrm{~s}$ following odor offset (Post Odor). There are no significant differences between groups at any of the 3 odor windows and there is no change in STC ensemble correlation after conditioned (relative to before conditioning) for either Control (blues) or Paired (reds) mice, suggesting olfactory fear conditioning and passive odor experience do not alter identity coding in STCs. Data displayed as mean $\pm \mathrm{SEM},{ }^{*} \mathrm{p}<$ 0.05 . 
Figure 4-6. Mitral cells demonstrate enhanced salience coding following olfactory fear conditioning.

(A) Activity heat maps of all defined MC-odor pairs in awake Control mice before (left) and after (right) training to E5, E4, and BZ. Individual cells are organized in rows and are matched between Pre and Post. White vertical lines demonstrate the odor-on window. (B) Quantification of odor-evoked fluorescence of Control mice in MCs from with inhibitory (left) and excitatory (right) response profiles during the odor-on window for all odors before and after conditioning. On average, Control mice demonstrate decreased odorevoked responses in excitatory profile MCs with little to no change in inhibitory profile MCs (C) Total percentage of Control MCs deemed either non-responsive after training as well as those deemed to have stronger (inhibitory or excitatory) or weaker (excitatory or inhibitory) responses after training than before. The majority of cells demonstrate weaker responses (weaker excitatory or weaker inhibitory) following control conditioning. (D) Heat maps of the activity of all defined STC-odor pairs in awake Paired mice before (left) and after (right) training to E5, E4, and BZ. Similar to Control mice, there is no significant difference in MCs with inhibitory response profiles following olfactory fear conditioning (E, left); however, Paired mice demonstrate increased odor-evoked responses in MCs with excitatory response profiles following fear learning (E, right). Most Paired MCs exhibit stronger (excitatory or inhibitory) responses following fear conditioning, with some displaying weaker (excitatory or inhibitory) responses (F). (G) Fold-change of Odor-evoked responses from Pre in Control (blue) and Paired (red) mice after clustering MCs into inhibitory or excitatory response profiles. $(\mathrm{H})$ Responses of excitatory MCs of Paired mice appear significantly stronger after olfactory fear conditioning to most odors. Data displayed as mean $\pm \mathrm{SEM},{ }^{*} \mathrm{p}<0.05$. 

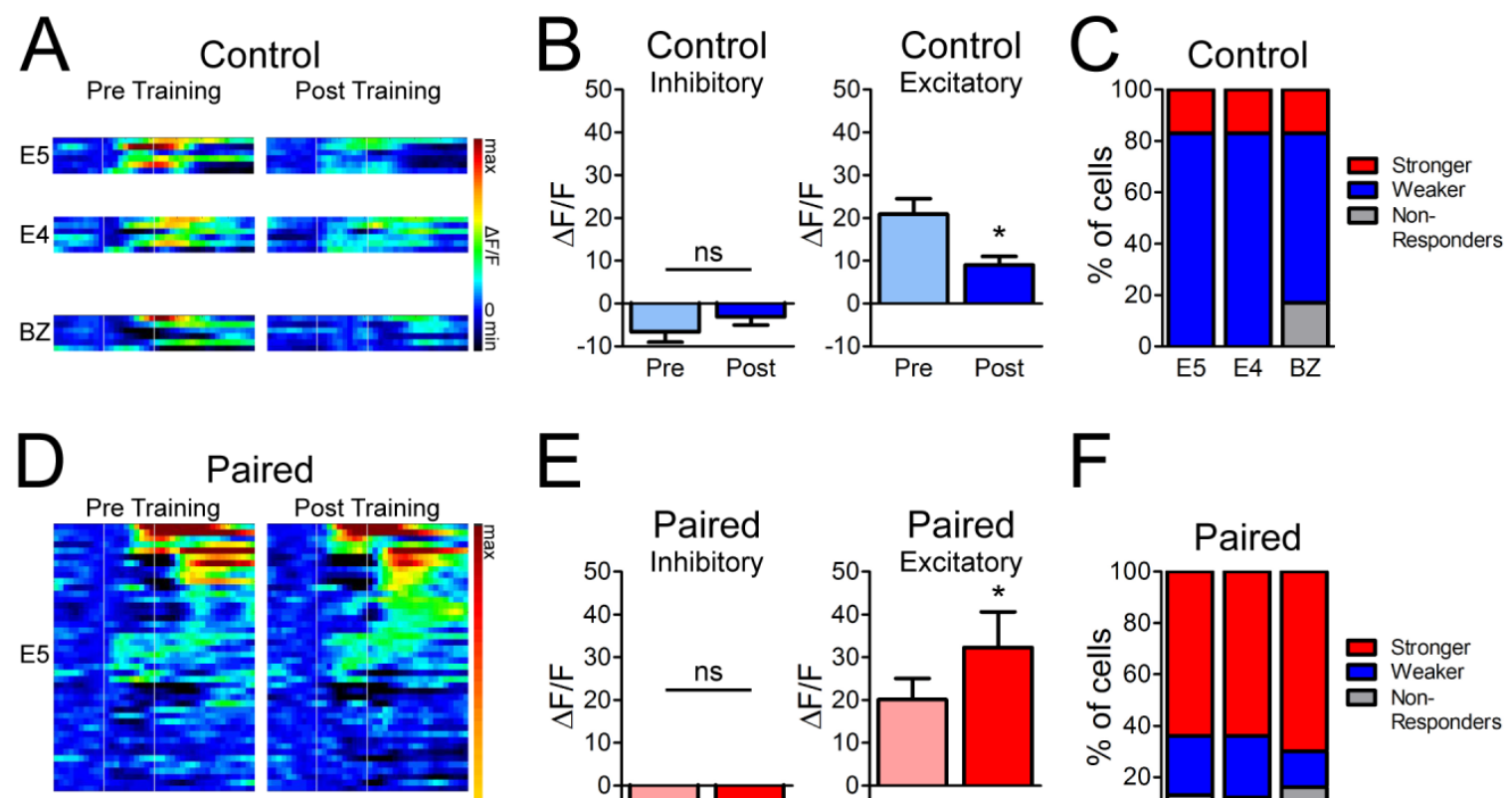

E
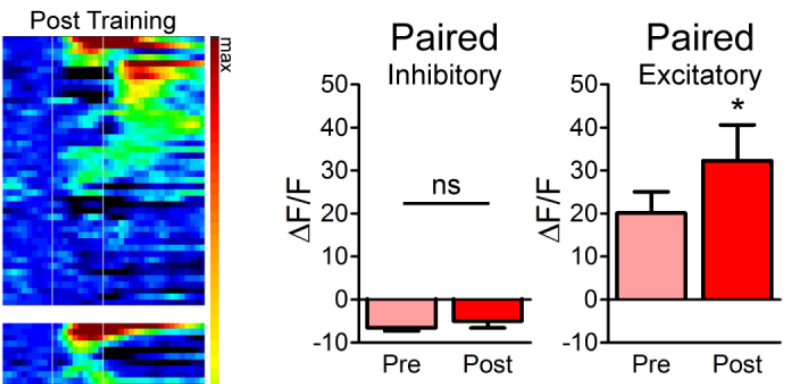

F
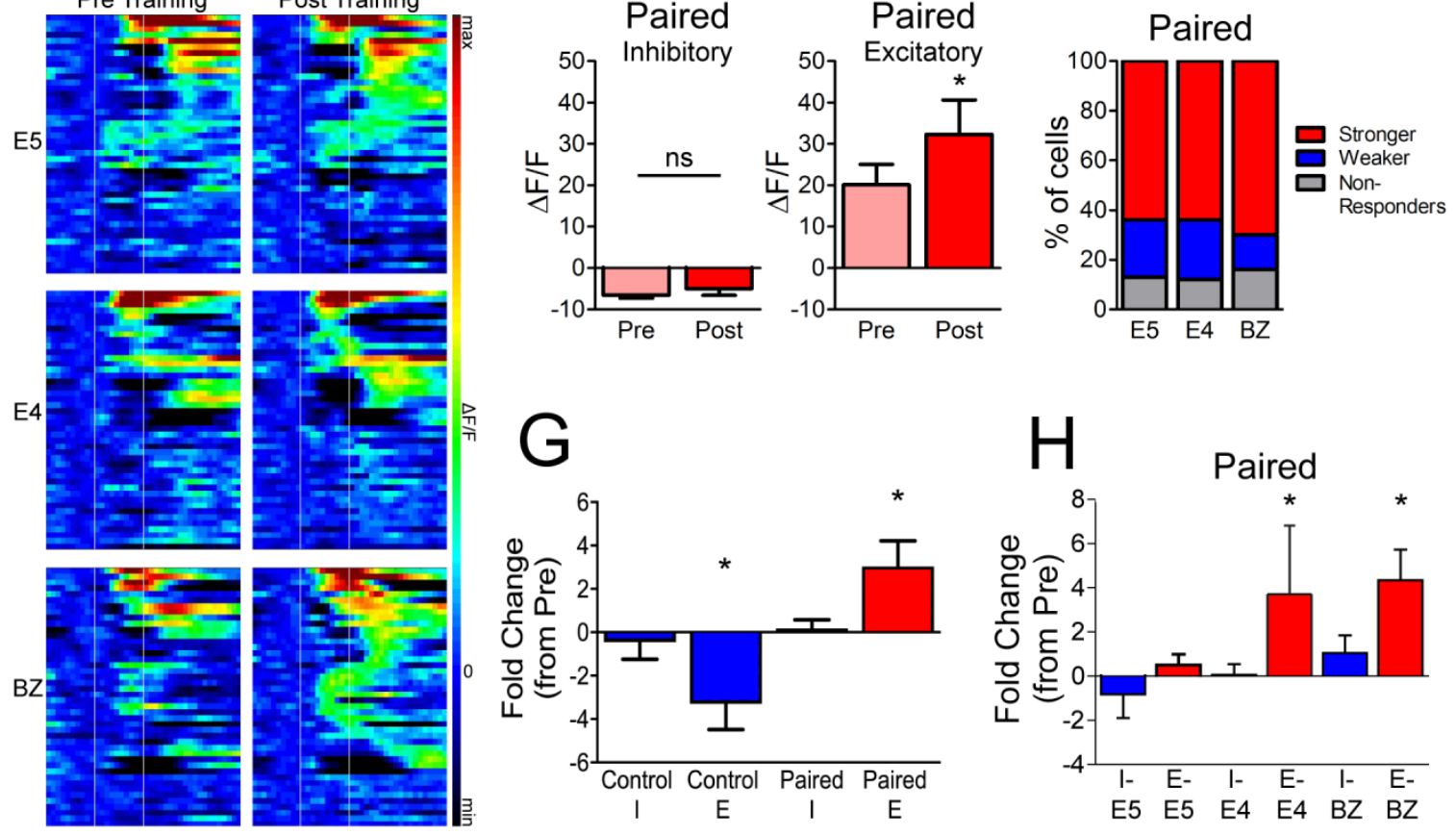
MCs odor-evoked responses are temporally dynamic and exhibit a wide range of amplitude responses (Nagayama et al., 2004); therefore, we used k-means cluster analysis to non-biasedly sort MCs into categories based on their response profiles (Figure 4-2). For analysis of changes during the odor-on window, we sorted cells into either excitatory (E) or inhibitory (I) response types (Figure 4-2B and 4-2D). Raw odor-evoked Control E $\mathrm{MC}$ responses were significantly weaker after conditioning (Figure 4-6B; $\mathrm{t}_{16}=3.818, \mathrm{p}=$ 0.0015; Mean E Pre $=20.90 \pm 3.600$, Mean E Post $=9.026 \pm 1.993)$, while Control I responses did not change significantly (Figure 4-6B; $\mathrm{t}_{10}=1.082, \mathrm{p}=0.3046$; Mean I Pre $=-6.621 \pm 2.422$, Mean I Post $=-3.108 \pm 1.924)$. Raw odor-evoked Paired E MC responses were also significantly altered following conditioning, but were stronger (Figure 4-6E; $\mathrm{t}_{93}=3.142, \mathrm{p}=0.0023$; Mean $\mathrm{E}$ Pre $=20.10 \pm 4.976$, Mean E Post $=32.30$ $\pm 8.311)$. As with Control I responses, Paired I MC responses did not change significantly as a result of odor-shock pairing (Figure 4-6E; $\mathrm{t}_{69}=1.182, \mathrm{p}=0.2411$; Mean I Pre $=-6.585 \pm 0.7422$, Mean I Post $=-5.013 \pm 1.601)$.

We also examined the fold change of E and I MCs for each group (Figure 4-6G). As expected based on raw quantifications, neither Control nor Paired conditioning experience significantly changes I MCs (Control: $\mathrm{W}^{*}=0.357, \mathrm{p}=0.721$; Paired: $\mathrm{W}^{*}=$ $0.558, \mathrm{p}=0.577)$. However, Control E MCs are $\sim 3$-fold weaker $\left(\mathrm{W}^{*}=-3.099, \mathrm{p}=0.002\right)$

and Paired E MCs are $\sim 3$-fold stronger $\left(\mathrm{W}^{*}=4.045, \mathrm{p}<0.0001\right)$ as a result of odor-shock pairing. The general trend of experience-dependent modulation where I MC responses are not changed and $\mathrm{E} \mathrm{MC}$ responses become weaker for Control mice and stronger for Paired MC does not change when looking at changes to specific odors (Figure 4-6G and 4-6H), suggesting all odor-evoked I-type responses become less inhibitory, regardless of conditioning experience and differences between Control and Paired mice are driven primarily by E-type responses. There are very few MCs that respond to only some of the tested odorants. Most MCs were completely unresponsive to our odors or responsive to each odor at some point during the odor-on or -off phases of imaging trials; therefore, we were unable to compare "Shared" vs "Unshared" responses.

\section{Olfactory Fear Conditioning Increases Odor-Evoked Mitral Cell Ensemble Similarity between CS and Neutral Odors}

We next wanted to investigate whether the spatiotemporal responses of $\mathrm{MC}$ populations change as a result of olfactory conditioning experience. The population correlations of Control mice were no different after control conditioning before $\left(\mathrm{t}_{5}=\right.$ $1.771, \mathrm{p}=0.137)$, during $\left(\mathrm{t}_{5}=0.256, \mathrm{p}=0.808\right)$, or after $\left(\mathrm{t}_{5}=0.945, \mathrm{p}=0.388\right)$ odor presentations (Figure 4-7A and 4-7C). However as a result of fear conditioning, the population correlations of Paired mice were significantly decorrelated before $\left(\mathrm{t}_{5}=3.059\right.$, $\mathrm{p}=0.028)$ and significantly more correlated during $\left(\mathrm{t}_{5}=-3.928, \mathrm{p}=0.011\right)$ and after $\left(\mathrm{t}_{5}=\right.$ $-3.481, \mathrm{p}=0.0 .18$ ) odor presentations (Figure 4-7B and 4-7C). This suggests that $\mathrm{MC}$ responses to neutral odors are more correlated to $\mathrm{MC}$ responses to the $\mathrm{CS}$ after fear conditioning than they were before. Furthermore, the increased similarity persists beyond the odor presentation. 

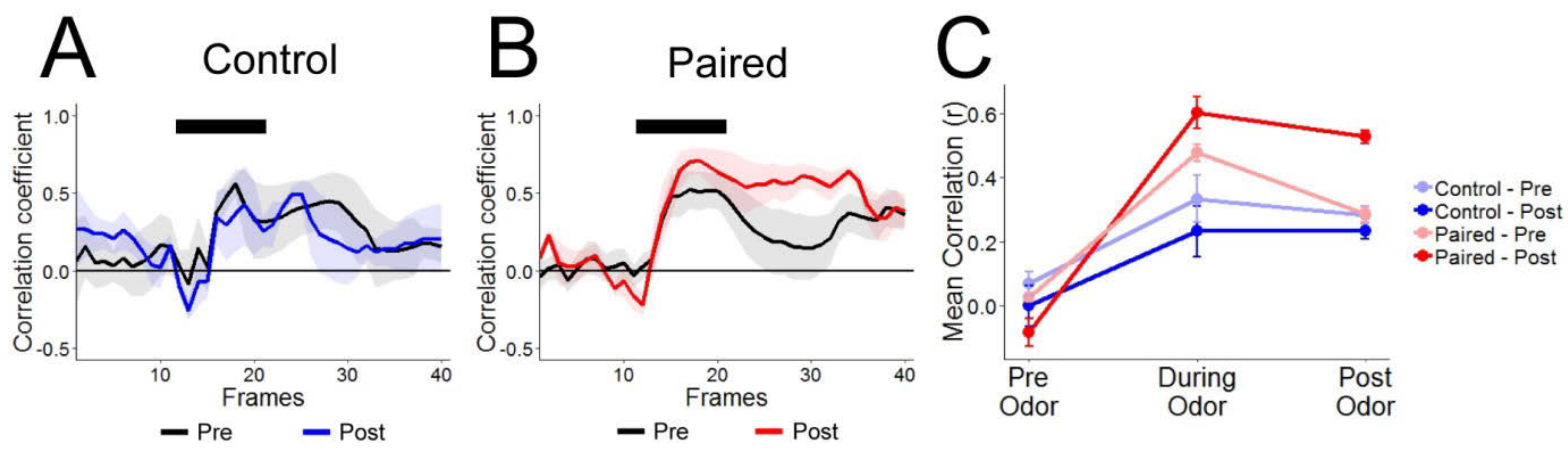

Figure 4-7. Neutral odor-evoked responses become more similar to conditioned odor-evoked responses in mitral cells.

(A-B) Traces of MC ensemble correlations between the CS and neutral odor responses in Control (A) and Paired (B) mice Pre (black) and Post (blue and red, respectively) conditioning. The correlation coefficients were calculated for the same MC population between pairs of CS-neutral odors at each time point and averaged. Black line indicates odor-presentation. (C) Quantification of average ensemble correlations between the CS and neutral odors for the $2 \mathrm{~s}$ before odor onset (Pre Odor) during the 2s odor presentation (During Odor) and for the $4 \mathrm{~s}$ following odor offset (Post Odor). There is no change in $\mathrm{MC}$ ensemble correlation of Control (blues) mice after conditioning (relative to before conditioning), suggesting passive odor experience does not alter odor identity coding in MCs of awake mice. Awake Paired (reds) mice demonstrate increased MC ensemble correlation between the CS and neutral odors following fear conditioning during the odor window (During Odor) that persists into the odor-off period (Post Odor), suggesting odor-evoked responses to neutral odors become more similar to the response evoked by the CS likely making it more difficult for awake mice to discriminate them. Data displayed as mean \pm SEM, $* \mathrm{p}<0.05$. 


\section{Discussion}

By pairing awake, 2P imaging with fear conditioning, we investigated the effects of fear learning on the two sets OB output cells responsible for transmitting sensory information to higher order brain processing centers. One theory of fear generalization suggests it arises from failure to perceptually discriminate the CS from similar but neutral stimuli; therefore, we also tested the extent to which fear learning alters neural responses in a way that might render organisms incapable of discriminating neutral odors from the CS. Our results demonstrate fear learning enhances evoked responses of both populations of output cells in distinct manners. The first population, STCs, is enhanced, possibly in a specific manner wherein cells that respond to the CS exhibit stronger learning-induced enhancements than those that do not respond to the CS. The second population, MCs, also displays learning-induced enhancement of odor-evoked responses but differ from STCs because they can be clustered into excitatory and inhibitory responses. Specifically, excitatory $\mathrm{MC}$ responses are enhanced by learning, whereas there is very little change in inhibitory MC responses. In both cell types, responses are altered for the CS as well as neutral odors, suggesting global changes in sensory coding at multiple levels of sensory processing. Additionally, we performed temporal correlation analysis to assess the population similarity of STCs and MCs responses to presentations of neutral odors and the CS before and after training. Interestingly, there was no change in temporal population correlations in STCs but a significant increase in MC population correlations during the odor presentation which persists after odor offset. This indicates MC odorevoked responses are altered by olfactory fear learning in a manner that makes responses to neutral odors more similar to the CS and could provide an initial neural basis for behavioral fear generalization by making odors more difficult to perceptually discriminate.

\section{Olfactory Fear Learning Changes Odor Coding in Output Cells in Distinct Ways}

We focused on the effects of olfactory fear learning on two populations of OB output neurons, STCs and MCs. There are a number of known anatomical and functional differences between STCs and MCs (Nagayama et al., 2004; Griff et al., 2008a; Griff et al., 2008b; Nagayama et al., 2010; Kikuta et al., 2013; Vaaga and Westbrook, 2016; Tavakoli et al., 2018), which could suggest that they play distinct roles in the perception of olfactory stimuli. We expand on these previous findings by identifying an additional functional difference following olfactory learning. While both STCs and MC exhibit stronger odor-evoked responses to a variety of odors following odor-shock pairing, only

MCs display increased correlation between the response evoked by neutral odors and the response evoked by the conditioned odor. This increased representational similarity signifies more generalized odor responsivity in MCs that likely makes it more difficult for mice to perceptually distinguish neutral odors from the CS and sets an initial neural basis for behavioral fear generalization. Together, this suggests that a major role of the MCs may be to convey information regarding odor identity to the rest of the brain. This differs from STCs, which do not appear to respond more similarly across odors following 
olfactory fear learning. Therefore, we propose that both populations of output cells signal salience about incoming olfactory information to the brain, allowing mice to better attend to odors by enhancing the odor-evoked responses, but only MCs code unique identity. The origin of this functional difference remains unknown but differences in excitatory and inhibitory input onto STCs and MCs (Burton, 2017) likely contributes to the divergent coding properties.

Nearly all STCs and MCs appear to have stronger odor-evoked responses across multiple odors following olfactory fear conditioning; however, MCs have varied response properties to odor presentations. Cluster analysis can separate MC odor-evoked responses into two clusters - one with a net excitatory response following odor onset and a second with a net inhibitory response following odor onset. Based on this clustering, we find that there is little to no change in the odor-evoked responses of the inhibitory cluster while the excitatory cluster exhibits stronger responses following olfactory fear conditioning. However, $\mathrm{MC}$ responses can also be clustered into five distinct clusters (Figure 4-2A and 4-2C). With this number of clusters, MCs fall into categories of 1) initial excitation during the odor that returns to baseline after the odor, 2) initial inhibition during the odor that returns to baseline after the odor, 3) initial excitation during the odor that persists after odor offset, 4) initial inhibition during the odor that persists after odor offset, and 5) initial inhibition during the odor that becomes excitation following odor offset. In addition, many of the cells do not fall into the same clusters following conditioning, suggesting robust changes in olfactory coding. We find that a significant proportion of cells $(\sim 30 \%)$ change polarity following olfactory fear conditioning, i.e., from inhibitory during odor presentation to excitatory or vice versa. Reanalyzing the MC data based on five clusters rather than two or focusing on so-called "polarity flippers" may bring additional insights to the robust reorganization of $\mathrm{MC}$ odor-evoked responses and how it relates to representational similarity of odorants.

\section{Potential Impact}

Foremost, we add to the existing evidence for several basic principles of learninginduced neural response alterations in sensory areas (Weinberger, 1995) associative learning alters primary stimulus representation, 2) altered stimulus representations exist at the level of single neurons and propagate to the larger population of neurons to change the representative stimulus map, 3) stimuli with acquired significance evoke stronger neural responses while stimuli lacking significance evoke weaker responses, and 4) altered neural responses exist at behaviorally relevant time points. We are able to extend and strengthen these basic principles by demonstrating their conservation in awake animals at the earliest stages of sensory encoding in the central nervous system.

Similar neural changes towards representing the CS have been reported in other sensory systems as well. Both auditory and sensory cortices expand representation of the $\mathrm{CS}$ and shift receptive fields towards the CS following classical aversive conditioning (Bakin and Weinberger, 1990; Scheich and Zuschratter, 1995; Bakin et al., 1996; Siucinska and Kossut, 1996; Weinberger and Bakin, 1998; Weinberger, 2004). 
Interestingly, changes in stimulus representation occur quickly and persist long after associative conditioning (Weinberger, 2007), providing a rapid and long lasting mechanism for altered sensory processing related to learning. Not only does this demonstrate massive reorganization of sensory system CS coding following fear learning but also suggests coding of neutral stimuli of the same sensory modality change as well. However, the extent to which aversive learning could transform neural responses such that presentations of neutral stimuli evoke responses more equivalent to presentations of CS was still poorly understood. Therefore, we specifically compared evoked responses of neutral stimuli and the CS in the same populations of neurons, OB STCs and MCs.

Importantly, the STCs and MCs are OB output cells, suggesting messages regarding incoming sensory information at the level of these cells are transmitted to higher-order processing centers that ultimately produce behavioral outputs following integration of relevant information. Together, our data suggest both populations of output cells likely signal increased salience of incoming sensory information following aversive learning via stronger odor-evoked neural responses. In addition, olfactory fear learning alters the coding of one population of output cells, MCs, in a way that increases response similarity between neutral odors and the conditioned odor. Odor coding in the OB reflects the structural and molecular identity of experienced odors (Wachowiak and Cohen, 2001; Spors and Grinvald, 2002; Bozza et al., 2004; Mori et al., 2006; Fletcher et al., 2009; Storace and Cohen, 2017); therefore, changes in coding presumably change the perception of experienced odors. Together this suggests that the increased representational similarity between neutral odors and the CS in MCs equates to increased perceptual similarity between neutral odors and the CS, which should make it more difficult to perceptually discriminate the experienced odors and lead to a failure of perceptual discrimination and, finally, behavioral fear generalization.

The present results illustrate that associative fear learning alters stimulus coding in primary sensory regions to increase salience and similarity between neutral and conditioned stimuli. These changes occur in output cells that project to additional processing centers, meaning they likely influence processing beyond the $\mathrm{OB}$ that contributes to behavioral responses. The results represent further evidence that behavioral fear generalization is not simply be a top-down process in which the brain employs a better safe-than sorry strategy. Instead, it appears that fear learning changes the coding in sensory regions in ways that increase representational similarity between neutral stimuli, to which fear is generalized, and the CS, which should be feared specifically. This mechanism, which points to a failure to perceptually discriminate between neutral and fear-significant stimuli, presents an interesting point of future study. As fear generalization is a hallmark of anxiety and trauma and stressor-related disorders (Cahill and Foa, 2007; Lissek et al., 2010; Lissek et al., 2014), understanding the sensory-based mechanisms that contribute to fear generalization may inform future treatments of this pathological behavior as well as our basic understanding of the mechanisms underlying long-term memory. 


\section{CHAPTER 5. OLFACTORY FEAR CONDITIONING-INDUCED REGULATION OF NEUROTRANSMITTER RECEPTOR GENE EXPRESSION IN THE OLFACTORY BULB}

\section{Introduction}

Olfactory fear conditioning involves pairing an initially neutral olfactory stimulus with a fear-inducing unconditioned stimulus, such as foot shock. The temporal pairing of these two stimuli ultimately causes an organism to learn an association between the olfactory stimulus and unconditioned stimulus. After pairing, the organism will begin to exhibit similar behavioral displays of fear during presentations of the olfactory stimulus as are elicited by the conditioned stimulus itself (Otto et al., 1997). This form of learning not only alters behavior but also modifies olfactory processing in the olfactory bulb (OB) as well as olfactory cortices (Freeman and Schneider, 1982; Sullivan and Wilson, 1991; Sullivan and Wilson, 1995; Sevelinges et al., 2004; Sevelinges et al., 2008; Rainecki et al., 2009; Chen et al., 2011; Fletcher, 2012; Kass et al., 2013; Kass and McGann, 2017; Ross and Fletcher 2018a). While the learning-induced OB response alterations may arise from centrifugal modulation, there is also evidence that the learning-induced changes may be intrinsic to the OB (Jones et al. 2008; Morrison et al. 2015), which suggests that there is some degree of convergence between the olfactory stimulus and unconditioned stimulus in the OB that alters olfactory processing.

Significant evidence indicates that olfactory learning induces OB plasticity that leads to altered OB processing (Brennan et al., 1998; Yuan et al., 2002; Sanchez-Andrade et al., 2005; Fletcher and Chen, 2010; Yuan et al., 2014). Many of the plastic changes appear to be in neurotransmitter receptors that regulate the excitability of OB cell types. For example, associative olfactory conditioning in rodents induces changes in glutamate receptors that increases mitral cell (MC) excitability to odor presentations and stabilizes both OB and cortical odor-evoked responses (Cui et al., 2011; Jerome et al., 2012; Lethbridge et al., 2012; Néant-Fery et al., 2012; Yuan et al., 2014; Shakhawat et al., 2015). Similarly, insects demonstrate intrinsic changes to glomeruli as well as enhanced glomerular responses following olfactory learning (Rath et al., 2011; Arenas et al., 2012). The exact source of these alterations in insects remains unknown; however, stimulation parameters meant to mimic olfactory associative learning induce long-term enhancement of synaptic transmission in olfactory areas via glutamate receptors as well as through receptors for other neurotransmitters (Kamimura et al., 2013).

There is significant neuromodulation in the mammalian OB. The OB receives centrifugal neuromodulation from fibers that original in neuromodulatory nuclei but also has signficiant, intrinsic neuromodulation from intrabulbar circuits, such as inhibitory interneurons (Shipley and Ennis, 1996; Fletcher and Chen 2010). Each neuromodulator works through a specific receptor or family of receptors to exert effects on neural activity. There is now significant evidence that olfactory learning alters OB processing and modulate neurotransmission. Given that neurotransmitter receptors regulate overall neural excitability and activity within OB circuits, the receptors represent an interesting 
avenue of investigation to identify potential mechanisms of learning-induced altered OB processing.

An understanding of learning-induced OB genetic regulation in neonatal rodents is well underway and seems to involve significant alterations in transcripts and protein receptors modulating neural transmissions (McLean et al., 1999; Zhang et al., 2003; Cui et al., 2007; Wang et al. 2013; Modarresi et al. 2016). However, little has been done to conduct a similarly thorough investigation of olfactory aversive learning-induced OB plasticity in adults. There are significant differences between the olfactory systems and learning of neonates and adults. Anatomically, the brain and synaptic connectivity continues to mature in the first postnatal weeks, especially in the olfactory system where most OB inhibitory networks are not fully developed for weeks after birth (RosselliAustin and Altman, 1979; Mair et al., 1982; Almli et al., 1985; Treloar et al., 2010). Behaviorally, at the neonatal timepoint, rodents do not demonstrate behavioral aversion following an olfactory aversive conditioning paradigm. Instead, neonatal rodents develop paradoxical preference to the aversive olfactory stimulus (Sullivan et al., 2000). While these differences do not preclude the same mechanisms from underlying both neonatal and adult $\mathrm{OB}$ plasticity, it suggests there may be differences in learning-induced OB plasticity at different developmental windows and that adult OB plasticity should be evaluated independently. Therefore, we aimed to perform an unbiased assay of gene expression of common neurotransmitter receptors in the $\mathrm{OB}$ of adult fear conditioned mice relative to controls.

Using the Qiagen RT2 Profiler Neurotransmitter Receptor PCR Array, we assessed the relative gene expression level of 84 different neurotransmitter receptors in the OBs of mice subjected to either a single day olfactory fear conditioning paradigm or control paradigms. We examined the gene expression levels at two different time points, 4 hours after conditioning, at a time that correlates with learning-induced gene expression regulation in other regions (Cavallaro et al., 2002; D'Agata and Cavallaro, 2003; Alberini and Kandel, 2014), as well as 24 hours after conditioning, when mice would generally be tested for olfactory fear learning. This design allowed for characterization of olfactory learning-induced gene expression changes in the adult $\mathrm{OB}$ during consolidation of learning and at a time point that coincides with typical expression. We find that relative gene expression of neurotransmitter receptors of mice exposed only to an odor and those exposed to foot shocks but naïve to odors do not differ significantly at either time point. However, mice that experienced odor paired with foot shock exhibit significant alterations in gene expression relative to controls, especially four hours after odor-shock experience. This represents the first attempt to characterize altered gene expression in an unbiased manner as a result of adult olfactory learning. Finally, we suggest possible mechanisms by which altered expression of the identified genes may impact olfactory coding after learning. 


\section{Methods and Materials}

\section{Animals}

Twenty adult (10 weeks) male C57B16/J mice (Jax Stock no: 000664) were used for fear conditioning and gene expression studies. Mice were maintained on a 12 hour light:dark schedule with ad libitum access to food and water except during behavioral experiments. Fear conditioning occurred during the light cycle (9am-11am) for all mice. Experimental protocols were in accordance with the University of Tennessee Institutional Animal Care and Use Committee.

\section{General Methodology}

\section{Olfactory fear conditioning}

Classical olfactory fear conditioning was performed as previously described (Ross and Fletcher 2018c). Briefly, mice were divided into three groups: 1 . Odor only $(n=6)$, 2. Shock only $(n=6)$, and 3. Paired $(n=8)$. Mice in the Odor only group were placed into a standard fear conditioning chamber (Coulbourn Instruments, cat. no: H10-11M) and received 6 unpaired presentations of ethyl valerate (E5; Sigma-Aldrich, cat. no: 290866) dissolved in mineral oil to a concentration of 200ppm. Mice in the Shock only group were placed into the same fear conditioning chamber and received 6 unpaired foot shocks $(0.8 \mathrm{ma}, 0.5 \mathrm{~s})$ delivered through a shock grid floor. Mice in the Paired group were placed into the fear conditioning chamber and received 6 presentations of E5 coterminating with foot shock. Mice were allowed to acclimate to the training environment for 10 minutes prior to the beginning of the training protocol. At the end of the training session, mice were removed from the training chamber and placed back into their home cage until tissue collection.

\section{Olfactory bulb tissue collection and preparation}

Four- or 24-hours after fear conditioning, mice were decapitated and OBs were collected. The left OB from each mouse was used for gene expression studies and the right OB was snap frozen and retained for protein expression studies. RNA was extracted and purified from OBs using the Qiagen RNeasy Mini Kit (Qiagen, cat. no: 74104). RNA was eluted to a total volume of $100 \mu 1$ for each OB and tested for concentration and purity via a Nanodrop spectrophotometer. All RNA samples had 260/280 ratios $>2.0$ and $260 / 230$ ratios of $>1.8$, indicating purity. Each purified template RNA sample was then diluted to achieve an equal total RNA load of 500ng for cDNA synthesis reactions. cDNA was synthesized using the RT2 First Strand Kit (Qiagen, cat. no: 330404). 


\section{Real time-polymerase chain reaction array}

Synthesized cDNA from each sample was mixed with the RT2 SYBR Green Mastermix and RNase free water. $25 \mu \mathrm{l}$ of the resultant mixture was added to each well of a 96-well RT2 Profiler PCR Array containing primers for 84 neurotransmitter receptors as well as 5 housekeeping genes (HKG) and 7 controls (Qiagen, cat. no: 330231; PAMM060ZA). Therefore, each plate contained the cDNA from a single mouse. Thermal cycling was performed using a Roche LightCycler 480 (LifeScience). Cycling conditions are as follows: initial denaturation phase at $95^{\circ} \mathrm{C}$ for 10 minutes plus 40 cycles of $95^{\circ} \mathrm{C}$ for $15 \mathrm{~s}$ followed by $60^{\circ} \mathrm{C}$ for $60 \mathrm{~s}$ with a $1.5^{\circ} \mathrm{C}$ ramp rate, according to manufacturer instructions. A fluorescence signal was acquired at the $60^{\circ} \mathrm{C}$ phase during each cycle. A melting curve program began immediately following the 40th cycle by increasing the temperature to $95^{\circ} \mathrm{C}$ and holding for $60 \mathrm{~s}$. All reactions resulted in only a single visible peak during melting curve analysis, indicating primer specificity in real time-polymerase chain reactions (RT-PCR).

\section{Quantification and Statistical Analyses}

A threshold was automatically set by the PCR collection software for each plate individually to define the point at which fluorescence RT-PCR traces for each gene transition to a linear amplification phase. Cycle threshold values (Ct values) were obtained for each gene reaction individually when the amplification curve crossed the defined threshold for a single plate. The raw $\mathrm{Ct}$ values for each of the five HKG (Actb, B2m, Gapdh, Gusb, Hsp90abl) were compared across all six groups (Odor only 4H, Shock only $4 \mathrm{H}$, Paired $4 \mathrm{H}$, Odor only $24 \mathrm{H}$, Shock only $24 \mathrm{H}$, and Paired $24 \mathrm{H}$ ) to determine the most stably expressed. Actb, the gene which codes for the cytoskeletal protein beta-actin, was determined to be the most stably expressed HKG with an average $\mathrm{Ct}$ value across all groups of $20.089 \pm 0.12(\mathrm{M}=19.98,20.28,19.93,20.01,19.76$, and 20.58 , respectively); therefore Actb was used to determine relative gene expression in all further analysis. Relative gene expression $(\Delta \mathrm{Ct})$ for each gene was calculated by subtracting the $\mathrm{Ct}$ value for a $\mathrm{HKG}(A c t b)$ from the $\mathrm{Ct}$ value for a gene of interest $\left(\mathrm{Ct}_{\mathrm{GOI}}\right.$ $-\mathrm{Ct}_{A c t b}$ ), such that values greater than 0 indicate genes that have higher $\mathrm{Ct}$ values and are therefore are less abundantly expressed than the HKG. Mean $\Delta \mathrm{Ct}$ were then calculated for each group (Odor only, Shock only, and Paired) by averaging the $\Delta \mathrm{Ct}$ for each gene of interest across animals in the same group. The mean $\Delta \mathrm{Ct}$ values were then statistically compared between groups to determine differences in relative gene expression for each gene of interest (Goni et al., 2009; Pfaffl, 2001).

All statistical analyses were performed on the $\Delta \mathrm{Ct}$ values using either Prism software (GraphPad, version 5.03) or SPSS (IBM, version 22.0). Assumptions of equal variances were first assessed with Levene's test for equality of variances. Most data did not exhibit significantly different variances between comparison groups; therefore, data were analyzed with parametric independent samples t-tests. If variances were found to differ, corrected $p$ values were reported. Genes were defined as "regulated" $0.10 \geq p \geq$ 0.05 , with statistical significance defined as $p \leq 0.05$. For brevity, $p$ values for every gene 
are reported along with descriptive data for Paired and Control mice in tables representing the 4 and 24 hour data points. All data are presented as mean \pm SEM unless otherwise noted.

The relative expression of each gene ( $\Delta \mathrm{Ct}$ values) was first compared between Odor only and Shock only groups at each time point (4 hours or 24 hours post training). Relative expression was only found to differ significantly between the two groups for Oxtr at 24 hours post training $(\mathrm{t} 4=4.263, \mathrm{p}=0.013)$; therefore, Odor only and Shock only groups were combined to become a Control group for further analysis and were statistically compared to the Paired group. Within these two groups the difference in mean $\Delta \mathrm{Ct}$ values for each gene was calculated between groups to obtain $\Delta \Delta \mathrm{Ct}$ values ( $\Delta \mathrm{Ct}_{\text {Paired }}-\Delta \mathrm{Ct}_{\text {Control }}$ ), to provide a measure of the difference in relative expression of genes of interest between groups. Calculations of fold change in mRNA expression between Paired and Control groups were obtained using $2^{-\Delta \Delta \mathrm{Ct}}$ method (Livak and Schmittgen 2001).

\section{Results}

\section{Odor-Shock Pairing Significantly Modifies Gene Expression 4 Hours After Experience}

We first sought to compare the transcription of neurotransmitter receptor genes between odor-shock pairing and controls (receiving only odor or shock) shortly after experience. Therefore, we isolated the OBs exactly four hours after each mouse finished the conditioning session and compared the expression of 84 different genes of interest between the two different experiences (Paired vs Control). Of the 84 genes (Table 5-1), 23 genes were determined to be differentially regulated $(\mathrm{p}<0.10)$ between Paired and Control conditioned mice with 13 of them statistically different (Figure 5-1).

Interestingly, the majority of these regulated genes were downregulated $(n=21)$ in the Paired conditioning group, suggesting odor-shock pairing mainly decreases gene transcripts of neurotransmitter receptors.

The regulated genes came from various gene families: acetylcholine receptors, dopamine receptors, GABA receptors, glutamate receptors, and serotonin receptors (Figure 5-2). The largest change in terms of relative expression was in the serotonin receptor, Htr 2c. Htr $2 c$ was upregulated $\sim 4.25$ fold in mice which experienced odor paired with shock. However, all other regulated serotonin receptors were downregulated. The only other gene upregulated in Paired animals 4 hours after conditioning was the glutamate receptor Grik4, which was upregulated $\sim 1.78$ fold. Again, all other regulated glutamate receptors were downregulated. All of the genes identified as being regulated following odor-shock pairing belonging to acetylcholine, dopamine, and GABA receptor families were downregulated at least $\sim 1.3$ fold. 
Table 5-1. Differences in relative OB gene expression between Control and Paired animals 4 hours after training.

\begin{tabular}{|c|c|c|c|c|}
\hline Gene family & Gene & $\begin{array}{c}\text { Control relative } \\
\text { expression } \\
\text { Mean } \pm \text { SEM }\end{array}$ & $\begin{array}{c}\text { Paired relative } \\
\text { expression } \\
\text { Mean } \pm \text { SEM }\end{array}$ & $p$ \\
\hline \multirow{5}{*}{ Adrenergic receptors } & Adrala & $4.804 \pm 0.2216$ & $5.240 \pm 0.1702$ & 0.180 \\
\hline & Adrald & $6.822 \pm 0.2567$ & $7.053 \pm 0.2066$ & 0.523 \\
\hline & Adra2a & $6.684 \pm 0.2515$ & $6.705 \pm 0.2382$ & 0.954 \\
\hline & Adrb2 & $8.872 \pm 0.2371$ & $9.283 \pm 0.2536$ & 0.278 \\
\hline & Adrb3 & $10.276 \pm 0.2811$ & $10.603 \pm 0.1970$ & 0.398 \\
\hline \multirow[t]{2}{*}{ Vasopressin receptors } & Avprla & $10.978 \pm 0.2188$ & $11.390 \pm 0.2841$ & 0.280 \\
\hline & Avprlb & $20.062 \pm 0.1221$ & $19.448 \pm 0.801$ & 0.501 \\
\hline Bombesin receptor & Brs 3 & $18.884 \pm 0.6754$ & $19.238 \pm 0.957$ & 0.765 \\
\hline Cholecystokinin receptor & $C C k b r$ & $6.446 \pm 0.1870$ & $6.653 \pm 0.1414$ & 0.428 \\
\hline \multirow[t]{9}{*}{ Acetylcholine receptors } & Chrm 1 & $3.536 \pm 0.2170$ & $3.960 \pm 0.1808$ & 0.191 \\
\hline & Chrm 4 & $5.162 \pm 0.1853$ & $5.853 \pm 0.1371$ & $0.025^{*}$ \\
\hline & Chrm5 & $12.054 \pm 0.2137$ & $12.365 \pm 0.1165$ & 0.276 \\
\hline & Chrna3 & $7.592 \pm 0.1649$ & $8.263 \pm 0.1176$ & $0.016^{*}$ \\
\hline & Chrna4 & $9.646 \pm 0.2782$ & $9.370 \pm 0.3714$ & 0.562 \\
\hline & Chrna5 & $10.864 \pm 0.1818$ & $11.735 \pm 0.1312$ & $0.008 *$ \\
\hline & Chrna6 & $13.758 \pm 0.2041$ & $14.523 \pm 0.4296$ & 0.128 \\
\hline & Chrna7 & $7.286 \pm 0.1989$ & $7.518 \pm 0.1638$ & 0.415 \\
\hline & Chrne & $10.116 \pm 0.1515$ & $10.495 \pm 0.0670$ & $0.075^{\wedge}$ \\
\hline Cannabinoid receptor & Cnrl & $6.162 \pm 0.2379$ & $5.768 \pm 0.3784$ & 0.387 \\
\hline \multirow[t]{3}{*}{ Dopamine receptors } & $\operatorname{Drdl}$ & $9.256 \pm 0.1345$ & $9.740 \pm 0.1844$ & $0.066^{\wedge}$ \\
\hline & $\operatorname{Drd} 2$ & $9.954 \pm 0.1677$ & $10.540 \pm 0.2117$ & $0.063^{\wedge}$ \\
\hline & $\operatorname{Drd5}$ & $11.860 \pm 0.2354$ & $12.130 \pm 0.0880$ & 0.364 \\
\hline \multirow[t]{17}{*}{ GABA receptors } & Gabbrl & $3.046 \pm 0.1824$ & $3.190 \pm 0.1634$ & 0.585 \\
\hline & Gabbr2 & $8.472 \pm 0.1404$ & $7.983 \pm 0.2612$ & 0.123 \\
\hline & Gabral & $2.726 \pm 0.1746$ & $3.110 \pm 0.1442$ & 0.146 \\
\hline & Gabra2 & $4.848 \pm 0.1698$ & $5.293 \pm 0.1125$ & $0.079^{\wedge}$ \\
\hline & Gabra4 & $6.152 \pm 0.1060$ & $6.595 \pm 0.1344$ & $0.034 *$ \\
\hline & Gabra5 & $3.570 \pm 0.1681$ & $4.245 \pm 0.1534$ & $0.023^{*}$ \\
\hline & Gabra6 & n.d. & n.d. & - \\
\hline & Gabrbl & $5.252 \pm 0.1097$ & $6.252 \pm 0.5937$ & 0.115 \\
\hline & Gabrb3 & $2.260 \pm 0.1522$ & $3.063 \pm 0.1285$ & $0.006^{*}$ \\
\hline & Gabrd & $6.288 \pm 0.1407$ & $6.478 \pm 0.1816$ & 0.429 \\
\hline & Gabre & $11.450 \pm 0.2520$ & $11.835 \pm 0.1698$ & 0.271 \\
\hline & Gabrg1 & $6.482 \pm 0.1627$ & $6.550 \pm 0.1866$ & 0.791 \\
\hline & Gabrg2 & $3.120 \pm 0.1137$ & $3.570 \pm 0.0964$ & $0.022 *$ \\
\hline & Gabrg3 & $7.316 \pm 0.1603$ & $8.358 \pm 0.4017$ & $0.034^{*}$ \\
\hline & Gabrq & $9.268 \pm 0.1962$ & $9.845 \pm 0.1432$ & $0.059^{\wedge}$ \\
\hline & Gabrr1 & $11.886 \pm 0.1462$ & $12.675 \pm 0.4209$ & $0.093^{\wedge}$ \\
\hline & Gabrr2 & $10.928 \pm 0.1851$ & $11.248 \pm 0.2242$ & 0.304 \\
\hline Glucagon receptor & $G c g r$ & $13.274 \pm 0.2271$ & $13.830 \pm 0.3017$ & 0.176 \\
\hline \multirow{3}{*}{$\begin{array}{c}\text { Glutamate receptors } \\
\text { (AMPARs) }\end{array}$} & Grial & $1.394 \pm 0.1030$ & $1.868 \pm 0.3268$ & 0.170 \\
\hline & Gria2 & $1.832 \pm 0.1393$ & $2.395 \pm 0.1307$ & $0.024 *$ \\
\hline & Gria3 & $4.280 \pm 0.2517$ & $4.345 \pm 0.1330$ & 0.839 \\
\hline
\end{tabular}


Table 5-1. Continued.

\begin{tabular}{|c|c|c|c|c|}
\hline Gene family & Gene & $\begin{array}{c}\text { Control relative } \\
\text { expression } \\
\text { Mean } \pm \text { SEM } \\
\end{array}$ & $\begin{array}{c}\text { Paired relative } \\
\text { expression } \\
\text { Mean } \pm \text { SEM }\end{array}$ & $p$ \\
\hline \multirow{4}{*}{$\begin{array}{l}\text { Glutamate receptors } \\
\text { (KARs) }\end{array}$} & Grikl & $6.792 \pm 0.1849$ & $7.060 \pm 0.3688$ & 0.510 \\
\hline & Grik2 & $5.682 \pm 0.5617$ & $5.113 \pm 0.1417$ & 0.375 \\
\hline & Grik4 & $12.476 \pm 0.2017$ & $11.643 \pm 0.2448$ & $0.033 *$ \\
\hline & Grik5 & $11.158 \pm 0.1367$ & $11.120 \pm 0.3039$ & 0.905 \\
\hline \multirow{4}{*}{$\begin{array}{c}\text { Glutamate receptors } \\
\text { (NMDARs) }\end{array}$} & Grin1 & $2.926 \pm 0.2114$ & $2.643 \pm 0.0390$ & 0.208 \\
\hline & Grin2a & $4.600 \pm 0.1921$ & $4.658 \pm 0.1936$ & 0.841 \\
\hline & Grin $2 b$ & $4.138 \pm 0.1953$ & $4.510 \pm 0.2307$ & 0.255 \\
\hline & $\operatorname{Grin} 2 c$ & $6.792 \pm 0.2007$ & $6.675 \pm 0.1603$ & 0.675 \\
\hline \multirow{7}{*}{$\begin{array}{l}\text { Glutamate receptors } \\
\text { (mGluRs) }\end{array}$} & Grm1 & $4.006 \pm 0.2373$ & $4.525 \pm 0.0486$ & $0.094^{\wedge}$ \\
\hline & Grm3 & $5.032 \pm 0.2724$ & $5.458 \pm 0.1453$ & 0.244 \\
\hline & Grm4 & $3.662 \pm 0.3276$ & $4.170 \pm 0.0911$ & 0.200 \\
\hline & Grm5 & $4.050 \pm 0.2695$ & $4.480 \pm 0.1437$ & 0.235 \\
\hline & Grm6 & $16.068 \pm 0.6179$ & $16.583 \pm 0.2073$ & 0.467 \\
\hline & Grm7 & $3.782 \pm 0.3155$ & $4.255 \pm 0.1371$ & 0.250 \\
\hline & Grm8 & $5.826 \pm 0.2089$ & $6.430 \pm 0.0292$ & $0.039 *$ \\
\hline Gastrin receptor & Grpr & $14.644 \pm 0.3887$ & $14.755 \pm 0.4151$ & 0.852 \\
\hline Orexin receptor & Hcrtr 2 & $11.790 \pm 0.3175$ & $11.088 \pm 0.3298$ & 0.172 \\
\hline \multirow[t]{2}{*}{ Histamine receptors } & $H r h 1$ & $9.942 \pm 0.2782$ & $10.283 \pm 0.0576$ & 0.292 \\
\hline & Hrh4 & $15.440 \pm 0.2494$ & $16.040 \pm 0.2703$ & 0.148 \\
\hline \multirow[t]{10}{*}{ Serotonin receptors } & Htrla & $8.512 \pm 0.2649$ & $8.880 \pm 0.1131$ & 0.254 \\
\hline & Htrlb & $9.546 \pm 0.2193$ & $9.708 \pm 0.3296$ & 0.685 \\
\hline & Htrld & $9.014 \pm 0.2936$ & $9.390 \pm 0.1699$ & 0.337 \\
\hline & Htrlf & $5.596 \pm 0.2075$ & $6.513 \pm 0.2198$ & $0.020 *$ \\
\hline & $H t r 2 a$ & $6.340 \pm 0.1947$ & $6.868 \pm 0.1346$ & $0.073^{\wedge}$ \\
\hline & $H t r 2 b$ & $7.264 \pm 0.1847$ & $7.628 \pm 0.2018$ & 0.227 \\
\hline & $H \operatorname{tr} 2 c$ & $8.922 \pm 0.5189$ & $7.588 \pm 0.7617$ & $0.022 *$ \\
\hline & $H t r 3 a$ & $7.710 \pm 0.1730$ & $8.270 \pm 0.2321$ & $0.088^{\wedge}$ \\
\hline & Htr4 & $13.070 \pm 0.3942$ & $12.723 \pm 0.2927$ & 0.522 \\
\hline & $H t r 7$ & $6.900 \pm 0.2317$ & $7.540 \pm 0.1769$ & $0.074^{\wedge}$ \\
\hline \multirow[t]{2}{*}{ Neuropeptide Y receptors } & $N p y 2 r$ & $11.786 \pm 0.3718$ & $11.498 \pm 0.5578$ & 0.669 \\
\hline & Npy5r & $8.790 \pm 0.3887$ & $8.918 \pm 0.0950$ & 0.784 \\
\hline \multirow[t]{2}{*}{ Neurotensin receptor } & Ntsr2 & $3.834 \pm 0.0485$ & $3.900 \pm 0.1773$ & 0.701 \\
\hline & Oxtr & $11.398 \pm 0.3419$ & $11.508 \pm 0.2183$ & 0.807 \\
\hline Prokineticin receptor & Prokr2 & $7.054 \pm 0.3617$ & $6.970 \pm 0.1003$ & 0.847 \\
\hline \multirow[t]{3}{*}{ Somatostatin receptors } & Sstrl & $9.732 \pm 0.3146$ & $9.420 \pm 0.2493$ & 0.480 \\
\hline & Sstr 2 & $10.466 \pm 1.0852$ & $9.060 \pm 0.1831$ & 0.295 \\
\hline & Sstr4 & $8.266 \pm 0.4985$ & $7.453 \pm 0.2889$ & 0.231 \\
\hline \multirow[t]{3}{*}{ Tachykinin receptors } & Tacrl & $7.664 \pm 0.2440$ & $7.688 \pm 0.2079$ & 0.945 \\
\hline & Tacr2 & $14.560 \pm 0.1501$ & $15.013 \pm 0.3093$ & 0.201 \\
\hline & Tacr3 & $11.750 \pm 0.1074$ & $12.845 \pm 0.7199$ & 0.226 \\
\hline Translocator protein & Tspo & $9.762 \pm 0.2108$ & $10.098 \pm 0.2508$ & 0.343 \\
\hline
\end{tabular}

$\mathrm{SEM}=$ standard error of the mean; ${ }^{*} \mathrm{p}<0.05 ;^{\wedge} 0.05<\mathrm{p}<0.1 ;$ n.d. indicates that transcript was not detected 


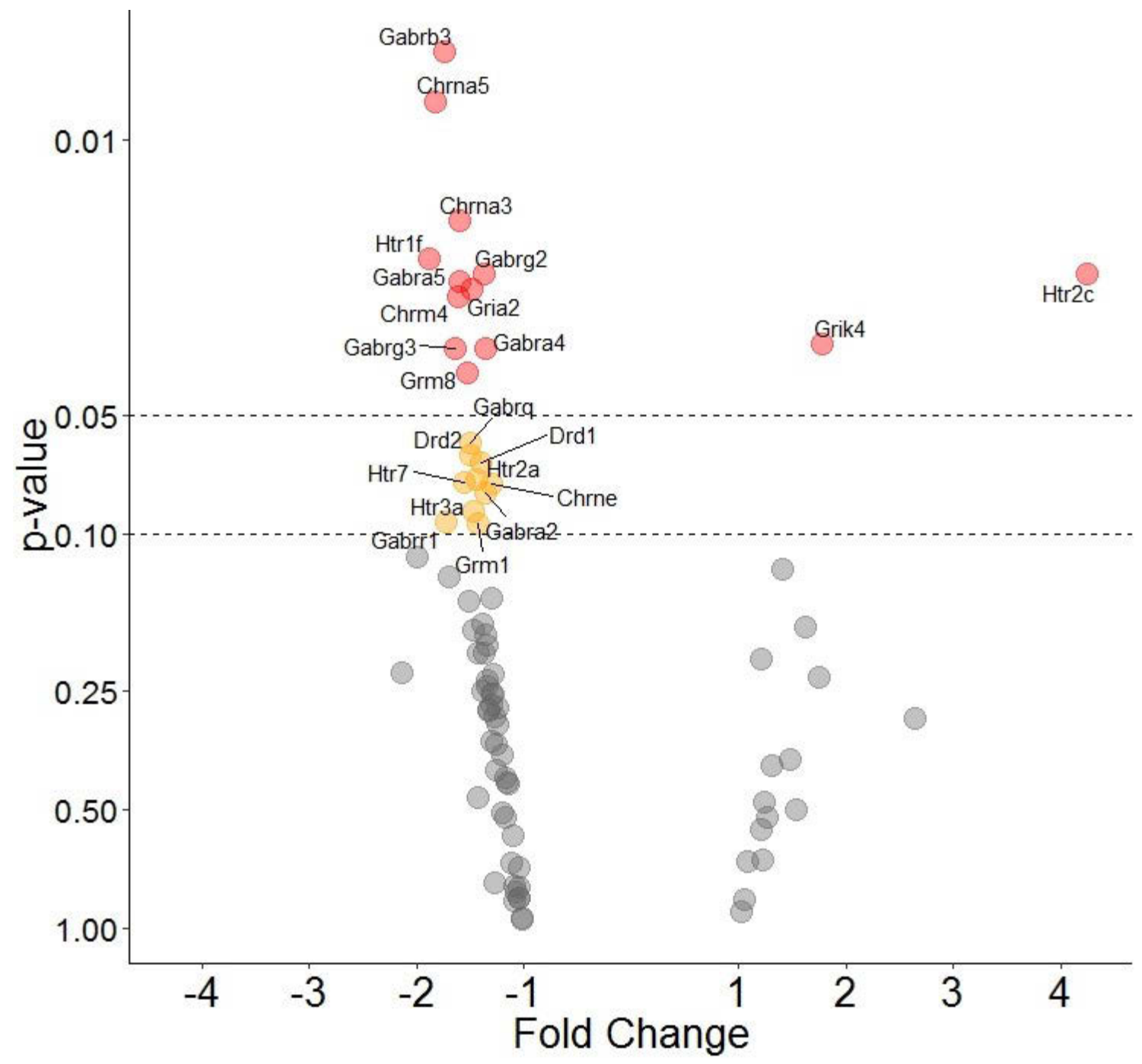

Figure 5-1. Relative $O B$ fold change of all neurotransmitter receptor genes in Paired mice compared to Controls 4 hours after olfactory fear conditioning. The relative fold change in the expression of all 84 assessed genes and their statistical significance. Grey dots signify genes that were not determined to be differentially regulated as a result of odor-shock experience, yellow dots represent genes that were determined to be differentially regulated as they reached statistical significance $0.10>p$ $>0.05$, red dots indicate genes that were determined to be differentially regulated as they reached statistical significance $\mathrm{p}<0.05$. Dotted lines illustrate the statistical significance cutoffs. Gene names appear next to genes determined to be differentially regulated. 


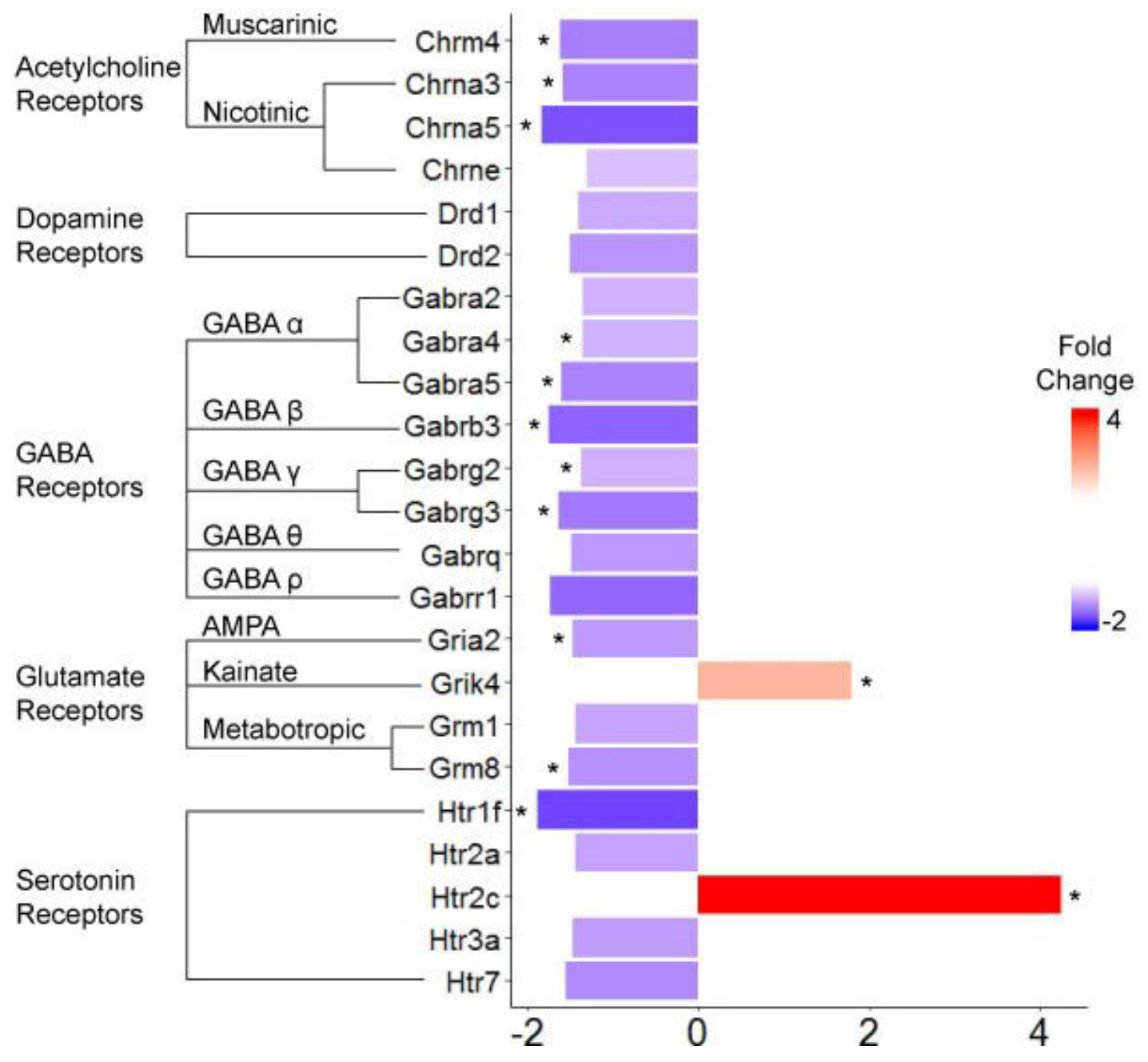

Figure 5-2. Paired experience $O B$ gene expression of regulated genes 4 hours after olfactory fear conditioning.

The average relative fold-changed of genes determined to be differentially regulated between Paired mice and Control mice 4 hours after conditioning. Bars are colored red and blue to indicate up- and downregulation of gene transcripts, respectively and the darkness of the bar indicates strength of regulation.* denote those genes that achieved statistical significance of $p<0.05$ while others were $0.05>p>0.10$. Dendrogram to left illustrates the gene families of each regulated gene. 


\section{Twenty-Four Hours After Odor-Shock Pairing, Gene Expression Changes Are Mostly Normalized}

Typically mice would be tested for expression of learned fear 24 hours after the conditioning experience; therefore, we were also interested to determine which, if any, genes for neurotransmitter receptors might be regulated in the $\mathrm{OB}$ at the comparable time point. We again isolated OBs from both Paired and Control mice, this time 24 hours after the conditioning experience, in order to compare the expression of the same 84 genes of interest between the two groups. Of the 84 genes (Table 5-2), only 8 were determined to be differentially regulated $(\mathrm{p}<0.10)$, with 5 achieving statistical significance (Figure 5-3), between Paired and Control conditioned mice.

As before, the regulated genes came from various families (Figure 5-4) but none of the genes differentially regulated at 4 hours were still differentially regulated at 24 hours with the exception of the dopamine receptor Drdl, which is $\sim 1.74$ fold downregulated 24 hours after training. Two acetylcholine receptor genes, Chrm 4 and Chrna3, were downregulated $\sim 1.6$ fold 4 hours after training but upregulated $\sim 1.75$ fold 24 hours after. Interestingly, two transcripts from the somatostatin family of gene receptors were significantly downregulated $\sim 1.6$ fold 24 hours post odor-shock pairing but were not different between Paired and Control mice 4 hours after training.

\section{Discussion}

\section{Potential Caveats}

It is known that gene transcription and subsequent translation into functional protein is required for the formation of stable memories after learning (Alberini and Kandel, 2014); however, the exact time course of learning-induced transcription appears to differ across brain regions and genes. Members of a class of genes called "immediate early genes" are the earliest genes to undergo transcriptional upregulation, which typically occurs beginning immediately during a learning event and are reduced by approximately 2 hours post learning (Cullinan et al., 1995). The immediate early genes are often transcriptional factors that impact transcriptional regulation of other genes; therefore, we chose 4 hours post-conditioning as our earliest time point to look for changes in neurotransmitter receptor transcripts. However, it is possible that 4 hours is too early and 24 hours too late of a time point to capture all of the changes that take place. Therefore, assessing gene expression at additional time points could provide more information about transcriptional regulation.

In this experiment, we looked at the relative gene expression of several neurotransmitter receptors; however, we did not investigate protein expression of the same receptors. Although genes encode proteins and upregulation of gene transcripts are generally concomitant with an increase in protein (Edfors et al., 2016), there is not necessarily a linear 1:1 ratio of gene transcripts to functional proteins (Silva and Vogel, 
Table 5-2. Differences in relative $O B$ gene expression between Control and Paired animals 24 hours after training.

\begin{tabular}{|c|c|c|c|c|}
\hline Gene family & Gene & $\begin{array}{c}\text { Control relative } \\
\text { expression } \\
\text { Mean } \pm \text { SEM }\end{array}$ & $\begin{array}{c}\text { Paired relative } \\
\text { expression } \\
\text { Mean } \pm \text { SEM }\end{array}$ & $p$ \\
\hline \multirow{5}{*}{ Adrenergic receptors } & Adrala & $4.978 \pm 0.2005$ & $5.037 \pm 0.0273$ & 0.786 \\
\hline & Adrald & $6.986 \pm 0.1874$ & $6.940 \pm 0.0503$ & 0.861 \\
\hline & Adra2a & $6.754 \pm 0.0900$ & $6.8367 \pm 0.0145$ & 0.518 \\
\hline & Adrb2 & $8.940 \pm 0.0826$ & $8.563 \pm 0.2706$ & 0.183 \\
\hline & Adrb3 & $10.264 \pm 0.1962$ & $10.370 \pm 0.1012$ & 0.710 \\
\hline \multirow[t]{2}{*}{ Vasopressin receptors } & Avprla & $11.248 \pm 0.3498$ & $11.410 \pm 0.1721$ & 0.749 \\
\hline & Avprlb & $18.110 \pm 0.7455$ & $17.583 \pm 0.4737$ & 0.764 \\
\hline Bombesin receptor & Brs3 & $18.391 \pm 0.7578$ & $16.390 \pm 1.0049$ & 0.160 \\
\hline Cholecystokinin receptor & $C C k b r$ & $6.598 \pm 0.0747$ & $6.430 \pm 0.2774$ & 0.611 \\
\hline \multirow[t]{9}{*}{ Acetylcholine receptors } & Chrml & $3.618 \pm 0.1112$ & $3.180 \pm 0.2747$ & 0.123 \\
\hline & Chrm4 & $5.188 \pm 0.1306$ & $4.463 \pm 0.3335$ & $0.052^{\wedge}$ \\
\hline & Chrm5 & $12.034 \pm 0.3344$ & $13.847 \pm 1.0083$ & $0.080^{\wedge}$ \\
\hline & Chrna3 & $8.028 \pm 0.7559$ & $7.143 \pm 0.3792$ & $0.024 *$ \\
\hline & Chrna4 & $9.760 \pm 0.2829$ & $10.045 \pm 0.0350$ & 0.372 \\
\hline & Chrna5 & $11.200 \pm 0.1972$ & $11.050 \pm 0.0200$ & 0.490 \\
\hline & Chrna6 & $14.034 \pm 0.2884$ & $13.525 \pm 0.2150$ & 0.369 \\
\hline & Chrna7 & $7.460 \pm 0.1182$ & $7.500 \pm 0.060$ & 0.850 \\
\hline & Chrne & $10.116 \pm 0.1495$ & $9.740 \pm 0.1554$ & 0.152 \\
\hline Cannabinoid receptor & Cnrl & $6.124 \pm 0.1948$ & $6.550 \pm 0.2100$ & 0.271 \\
\hline \multirow[t]{3}{*}{ Dopamine receptors } & $\operatorname{Drdl}$ & $9.614 \pm 0.1314$ & $10.417 \pm 0.3002$ & $0.029 *$ \\
\hline & $\operatorname{Drd} 2$ & $10.132 \pm 0.1212$ & $9.997 \pm 0.2270$ & 0.580 \\
\hline & $\operatorname{Drd5}$ & $12.070 \pm 0.2403$ & $12.055 \pm 0.1950$ & 0.973 \\
\hline \multirow[t]{17}{*}{ GABA receptors } & Gabbrl & $3.178 \pm 0.1539$ & $3.320 \pm 0.0100$ & 0.409 \\
\hline & Gabbr2 & $8.468 \pm 0.1905$ & $8.083 \pm 0.3023$ & 0.297 \\
\hline & Gabral & $3.020 \pm 0.1371$ & $2.498 \pm 0.3303$ & 0.217 \\
\hline & Gabra2 & $5.100 \pm 0.0570$ & $4.533 \pm 0.3721$ & 0.225 \\
\hline & Gabra4 & $6.014 \pm 0.2359$ & $5.790 \pm 0.1297$ & 0.466 \\
\hline & Gabra5 & $3.618 \pm 0.1488$ & $2.898 \pm 0.4548$ & 0.213 \\
\hline & Gabra6 & n.d. & n.d. & - \\
\hline & Gabrbl & $5.270 \pm 0.0430$ & $5.143 \pm 0.3054$ & 0.654 \\
\hline & Gabrb3 & $2.292 \pm 0.1142$ & $1.365 \pm 0.4743$ & 0.144 \\
\hline & Gabrd & $6.178 \pm 0.1510$ & $5.755 \pm 0.1784$ & 0.111 \\
\hline & Gabre & $11.372 \pm 0.1551$ & $11.125 \pm 0.3031$ & 0.464 \\
\hline & Gabrg1 & $6.338 \pm 0.0788$ & $6.393 \pm 0.2350$ & 0.815 \\
\hline & Gabrg2 & $3.294 \pm 0.0870$ & $3.273 \pm 0.0984$ & 0.885 \\
\hline & Gabrg3 & $7.218 \pm 0.0576$ & $7.210 \pm 0.1115$ & 0.945 \\
\hline & Gabrq & $9.354 \pm 0.0815$ & $9.070 \pm 0.1026$ & $0.064^{\wedge}$ \\
\hline & Gabrrl & $11.608 \pm 0.1877$ & $11.758 \pm 0.2209$ & 0.620 \\
\hline & Gabrr2 & $11.448 \pm 0.1807$ & $10.915 \pm 0.2454$ & 0.116 \\
\hline Glucagon receptor & $G c g r$ & $13.872 \pm 0.385$ & $13.680 \pm 0.1935$ & 0.673 \\
\hline Glutamate receptors & Grial & $1.222 \pm 0.0807$ & $1.037 \pm 0.0788$ & 0.180 \\
\hline \multirow[t]{2}{*}{ (AMPA) } & Gria2 & $1.770 \pm 0.1085$ & $1.883 \pm 0.1625$ & 0.567 \\
\hline & Gria3 & $3.944 \pm 0.1154$ & $0.368 \pm 0.2092$ & 0.313 \\
\hline
\end{tabular}


Table 5-2. Continued.

\begin{tabular}{|c|c|c|c|c|}
\hline Gene family & Gene & $\begin{array}{c}\text { Control relative } \\
\text { expression } \\
\text { Mean } \pm \text { SEM }\end{array}$ & $\begin{array}{c}\text { Paired relative } \\
\text { expression } \\
\text { Mean } \pm \text { SEM }\end{array}$ & $p$ \\
\hline \multirow{4}{*}{$\begin{array}{l}\text { Glutamate receptors } \\
\text { (Kainate) }\end{array}$} & Grikl & $6.330 \pm 0.138$ & $5.913 \pm 0.2054$ & 0.130 \\
\hline & Grik2 & $4.426 \pm 0.0787$ & $4.190 \pm 0.1926$ & 0.257 \\
\hline & Grik4 & $11.796 \pm 0.2741$ & $11.763 \pm 0.2307$ & 0.938 \\
\hline & Grik5 & $10.824 \pm 0.2342$ & $11.358 \pm 0.5339$ & 0.355 \\
\hline \multirow{4}{*}{$\begin{array}{l}\text { Glutamate receptors } \\
\text { (NMDA) }\end{array}$} & Grinl & $2.610 \pm 0.2636$ & $2.493 \pm 0.2267$ & 0.774 \\
\hline & Grin $2 a$ & $4.716 \pm 0.2122$ & $4.885 \pm 0.4069$ & 0.707 \\
\hline & Grin $2 b$ & $4.064 \pm 0.1408$ & $4.113 \pm 0.0384$ & 0.804 \\
\hline & Grin $2 c$ & $6.696 \pm 0.1573$ & $6.893 \pm 0.0731$ & 0.397 \\
\hline \multirow{7}{*}{$\begin{array}{l}\text { Glutamate receptors } \\
\text { (metabotropic) }\end{array}$} & Grm1 & $4.208 \pm 0.1566$ & $4.095 \pm 0.0817$ & 0.574 \\
\hline & Grm3 & $5.396 \pm 0.1006$ & $5.320 \pm 0.0557$ & 0.607 \\
\hline & Grm4 & $4.148 \pm 0.1558$ & $4.300 \pm 0.0907$ & 0.512 \\
\hline & Grm5 & $4.304 \pm 0.1109$ & $4.053 \pm 0.2118$ & 0.300 \\
\hline & Grm6 & $15.708 \pm 0.3390$ & $17.550 \pm 1.1263$ & 0.126 \\
\hline & Grm7 & $4.178 \pm 0.1187$ & $4.128 \pm 0.1249$ & 0.780 \\
\hline & Grm8 & $6.036 \pm 0.1510$ & $5.780 \pm 0.1547$ & 0.280 \\
\hline Gastrin receptor & $G r p r$ & $14.288 \pm 0.2151$ & $14.138 \pm 0.3556$ & 0.715 \\
\hline Orexin receptor & Hcrtr 2 & $11.798 \pm 0.2585$ & $12.243 \pm 0.3685$ & 0.343 \\
\hline \multirow[t]{2}{*}{ Histamine receptors } & Hrhl & $10.214 \pm 0.1835$ & $10.470 \pm 0.1225$ & 0.311 \\
\hline & $\mathrm{Hrh} 4$ & $15.552 \pm 0.2398$ & $14.363 \pm 0.8298$ & 0.133 \\
\hline \multirow[t]{10}{*}{ Serotonin receptors } & Htrla & $8.736 \pm 0.1629$ & $8.767 \pm 0.1071$ & 0.899 \\
\hline & $H t r l b$ & $9.678 \pm 0.1637$ & $9.658 \pm 0.1645$ & 0.933 \\
\hline & Htrld & $9.200 \pm 0.1262$ & $9.257 \pm 0.1053$ & 0.770 \\
\hline & Htrlf & $5.818 \pm 0.1419$ & $5.643 \pm 0.1434$ & 0.419 \\
\hline & $H$ tr $2 a$ & $6.610 \pm 0.1714$ & $6.647 \pm 0.1714$ & 0.857 \\
\hline & $H t r 2 b$ & $7.294 \pm 0.1369$ & $7.220 \pm 0.1372$ & 0.714 \\
\hline & $H t r 2 c$ & $8.644 \pm 0.7053$ & $9.713 \pm 0.2586$ & 0.214 \\
\hline & $H \operatorname{tr} 3 a$ & $7.866 \pm 0.1299$ & $7.633 \pm 0.2217$ & 0.371 \\
\hline & Htr4 & $12.524 \pm 0.923$ & $13.057 \pm 0.751$ & $0.008 *$ \\
\hline & $H t r 7$ & $7.320 \pm 0.1372$ & $7.188 \pm 0.0629$ & 0.449 \\
\hline \multirow[t]{2}{*}{ Neuropeptide Y receptors } & $N p y 2 r$ & $12.462 \pm 0.8677$ & $12.433 \pm 0.1848$ & 0.981 \\
\hline & $N p y 5 r$ & $8.652 \pm 0.1798$ & $8.660 \pm 0.1512$ & 0.975 \\
\hline \multirow[t]{2}{*}{ Neurotensin receptor } & Ntsr2 & $3.796 \pm 0.1202$ & $3.643 \pm 0.0240$ & 0.380 \\
\hline & Oxtr & $11.108 \pm 0.1987$ & $10.887 \pm 0.1378$ & 0.464 \\
\hline Prokineticin receptor & Prokr2 & $6.544 \pm 0.0800$ & $6.493 \pm 0.0133$ & 0.653 \\
\hline \multirow[t]{3}{*}{ Somatostatin receptors } & Sstr1 & $9.298 \pm 0.1095$ & $9.843 \pm 0.1523$ & $0.020^{*}$ \\
\hline & Sstr 2 & $9.580 \pm 0.1688$ & $9.797 \pm 0.0882$ & 0.390 \\
\hline & Sstr 4 & $7.428 \pm 0.0728$ & $8.173 \pm 0.3859$ & $0.046^{*}$ \\
\hline \multirow[t]{3}{*}{ Tachykinin receptors } & Tacrl & $7.430 \pm 0.0789$ & $7.303 \pm 0.0933$ & 0.351 \\
\hline & Tacr2 & $14.660 \pm 0.2547$ & $14.368 \pm 0.3647$ & 0.519 \\
\hline & Tacr3 & $12.020 \pm 0.1780$ & $11.748 \pm 0.3657$ & 0.496 \\
\hline Translocator protein & Tspo & $9.362 \pm 0.1595$ & $9.093 \pm 0.1844$ & 0.327 \\
\hline
\end{tabular}

$\mathrm{SEM}=$ standard error of the mean; ${ }^{*} \mathrm{p}<0.05 ;^{\wedge} 0.05<\mathrm{p}<0.1 ;$ n.d. indicates that transcript was not detected 


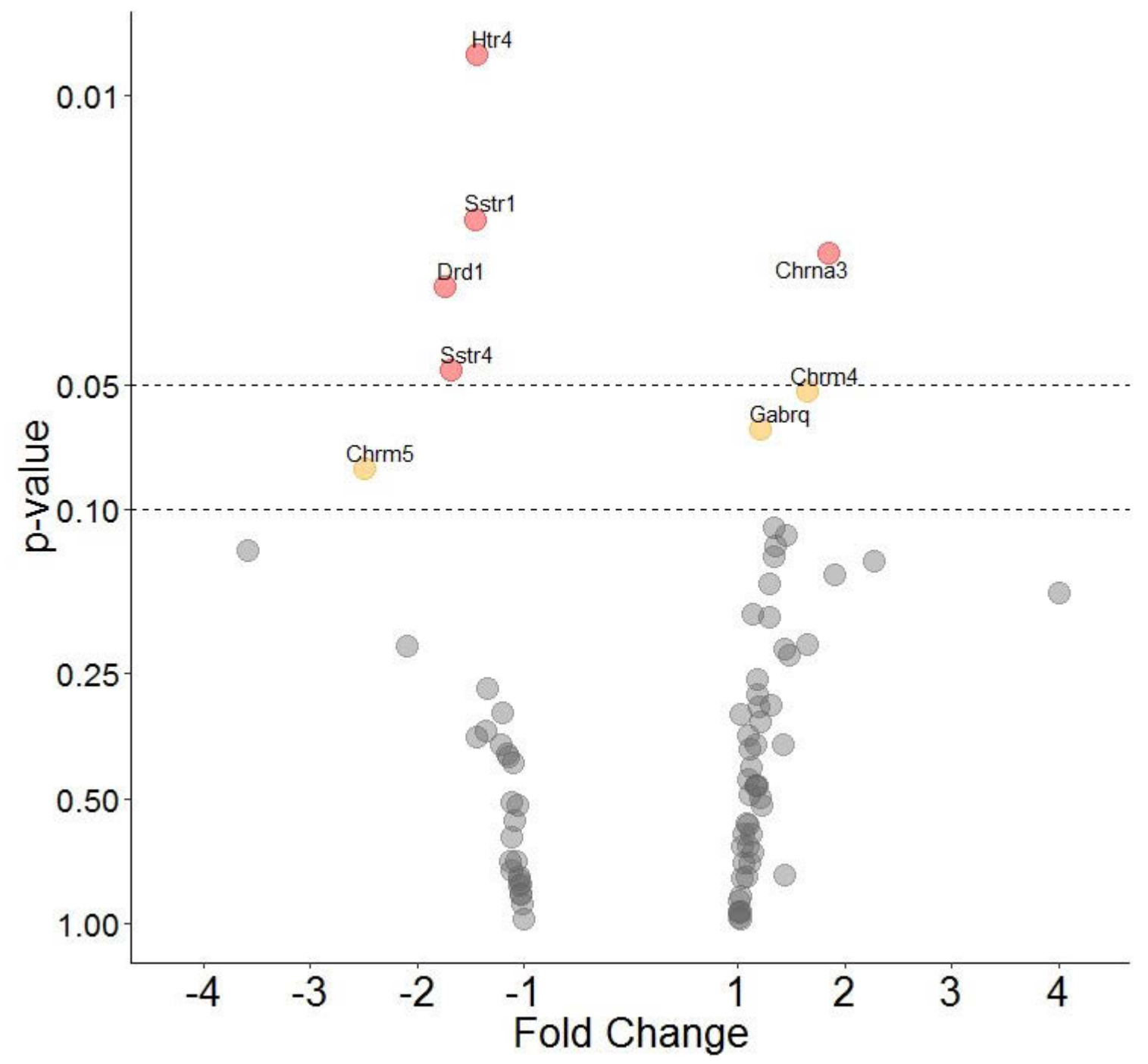

Figure 5-3. Relative $O B$ fold change of all neurotransmitter receptor genes in Paired mice compared to Controls 24 hours after olfactory fear conditioning.

The relative fold change in the expression of all 84 assessed genes and their statistical significance. Grey dots signify genes that were not determined to be differentially regulated as a result of odor-shock experience, yellow dots represent genes that were determined to be differentially regulated as they reached statistical significance $0.10>p$ $>0.05$, red dots indicate genes that were determined to be differentially regulated as they reached statistical significance $\mathrm{p}<0.05$. Dotted lines illustrate the statistical significance cutoffs. Gene names appear next to genes determined to be differentially regulated. 


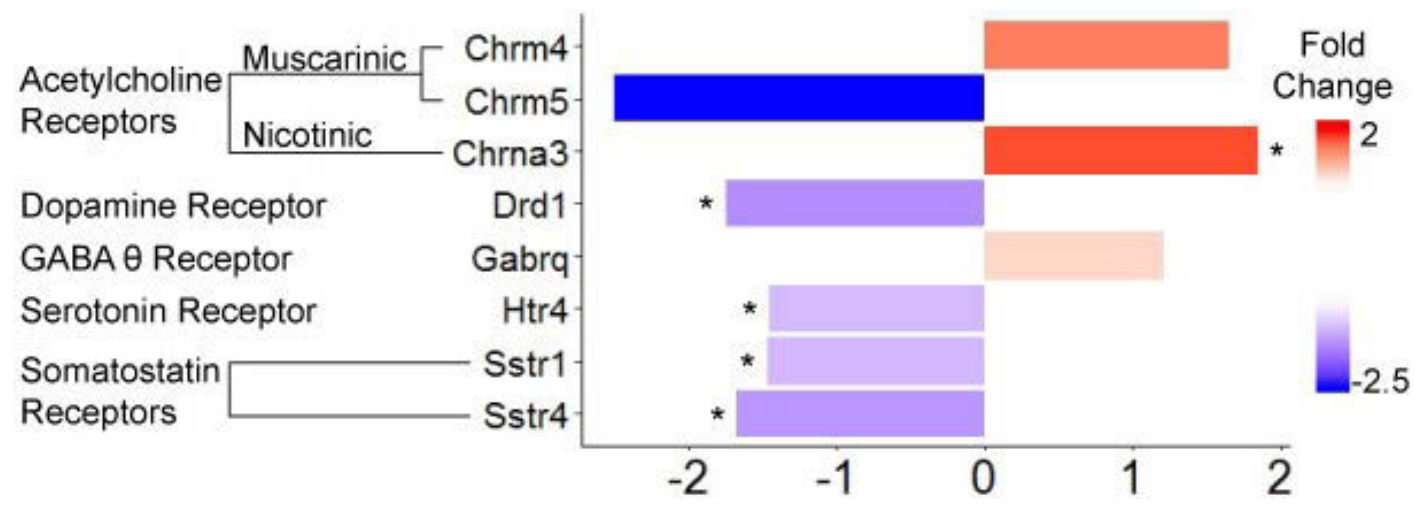

Figure 5-4. Paired-experience OB gene expression of regulated genes 24 hours after olfactory fear conditioning.

The average relative fold-changed of genes determined to be differentially regulated between Paired mice and Control mice 24 hours after conditioning. Bars are colored red and blue to indicate up- and downregulation of gene transcripts, respectively and the darkness of the bar indicates strength of regulation.* denote those genes that achieved statistical significance of $p<0.05$ while others were $0.05>p>0.10$. Dendrogram to left illustrates the gene families of each regulated gene. 
2016; Csardi et al., 2015) and the processes of translation, expression, and turnover can affect levels of protein expression (Maier et al., 2009). While there are a number of factors that can affect the correlation between mRNA and protein expression levels, the overall assumption that changes in mRNA have a biological meaning that often corresponds to changes in protein levels is still valid (Koussounadis et al., 2015). The results obtained here would be strengthened by exploring the extent to which olfactory fear conditioning affects the protein levels of the regulated genes.

In this study, we isolated mRNA from whole OBs; therefore, while we can assess global alterations in gene transcripts as a result of experience, we cannot distinguish specifically where expression changes take place. The mouse OB comprises many layers, some of which contain several distinct cell types that may be affected differently by the same fear conditioning experience. Therefore it is possible that there are several other differentially regulated gene transcripts is specific subsets of cells that cannot be identified in a whole OB isolation because there exists complimentary modulation of the same transcripts in a different cell population that masks the specific changes. For example, if a specific glutamate receptor were upregulated in MCs but downregulated in granule cells (GCs) to the same degree as a result of olfactory fear conditioning, a whole OB isolation would not detect any alterations in the amount of transcript relative to control mice. Alternatively, the same glutamate receptor could be upregulated in both MCs and GCs, which, while being a significant regulation of gene expression, may have no functional impact on the olfactory system. It will be necessary to use alternative techniques, such as cell sorting or in situ hybridization to determine cell-type or laminarspecific gene expression changes. This could also be done in concert with protein assays, using immunohistochemistry to determine where in the OB functional protein differences lie between paired and control conditions. Another option would be to use cell sorting techniques with either RNAseq or proteomic approaches. Without specific knowledge of the cell type/s in which gene expression is regulated, any discussion of the effects is, at best, speculative.

\section{Acetylcholine Receptors}

The OB receives significant cholinergic innervation from the horizontal limb of the diagonal band of Broca (Macrides et al., 1981; Zaborszsky et al., 1986; Levy et al., 1999). The majority of these cholinergic fibers terminate in the glomerular layer (GL), with a smaller percentage terminating in infra-glomerular layers (Shipley and Ennis. 1996; Case et al., 2017). There are two types of acetylcholine receptors (AChRs), nicotinic receptors (nAChRs) and muscarinic receptors (mAChRs), both of which are widely expressed in the OB (Le Jeune et al., 1995; Castillo et al., 1999; Ghatpande and Gelperin, 2009; D'Souza and Vijayaraghavan, 2012) and affect olfactory processing (Ravel et al., 1990; Elaagouby et al., 1991; Castillo et al., 1999; Liu et al., 2015; Smith et al., 2015; Case et al., 2017). Importantly, it is known that acetylcholine (ACh) is required for both pup and adult olfactory associative learning (Chaudhury et al., 2009; Kroon and Carobrez, 2009; Devore et al., 2012; Hellier et al., 2012; Pavesi et al., 2012; Chan et al. 2017) so it is unsurprising that gene expression of AChRs changes following olfactory 
fear conditioning. Our results demonstrate that four hours after olfactory fear conditioning, Chrm4, which codes for mAChR4, and Chrna3 and Chrna5, which code for $\mathrm{nAChR} 3$ and $\mathrm{nAChR} 5$ respectively, are downregulated. Interestingly, the gene expression of Chrna5 is still decreased 24 hours later, while expression of the Chrm 4 and Chrna3 genes are upregulated relative to controls.

In general, the role of $\mathrm{mAChRs}$ remains unclear in the $\mathrm{OB}$ and there is conflicting evidence regarding the role of $\mathrm{mAChRs}$ in GL processing. For example, one recent report suggests $m A C h R s$ recruit inhibitory GL circuits, which should decrease glomerular responses (Liu et al., 2015) while another demonstrates signaling through mAChRs enhances glomerular sensitivity to odor inputs (Bendahmane et al., 2016). Even less is known regarding specific subunits of $\mathrm{mAChRs}$ and their role in olfaction. With respect to mAChR4, it appears to mediate ACh-dependent stimulation of adenylyl cyclase and cAMP in the OB (Dittman et al., 1994; Onali et al., 1994) and is expressed on adult-born OB neurons (Kaneko et al., 2006), which suggests mAChR4 supports OB neurogenesis. In the context of our results, the downregulation 4 hours after training followed by upregulation 24 hours after could indicate a mechanism involving fear conditioninginduced neurogenesis or synaptogenesis, which would be in line with previous reports (Jones et al. 2008) though the short timescale may not support this conclusion. Other than that, relatively little is known about the role of mAChR4 in the OB. In other systems, it has been suggested that mAChR4 exerts inhibitory control over D1 receptor activity (Gomeza et al., 1999), which may provide an indirect role by which alterations in $\mathrm{mAChR} 4$ expression could modulate olfactory processing.

Activation of nAChRs is also thought to play a role in OB adult neurogenesis; however, this increased neurogenesis seems to correlate negatively with olfactory learning (Mechawar et al., 2004). The exact nAChR subtypes that contribute to neurogenesis and poor memory have yet to be identified so we cannot conclude whether downregulation of Chrna3 and Chrna5 is consistent with these findings. While the role of our identified genes and their function in olfactory learning is unknown, nAChRs presynaptically modulate glutamate release, which sculpts OB GL processing and output (Alkondon et al., 1996; Parsa et al., 2015; Bendahmane et al. 2016). This may be due to nAChRs containing the $\alpha 3$ subunit (coded for by Chrna3) as signaling through $\mathrm{nAChR} 3$ enhances activity of both excitatory output cells and GL interneurons to ultimately regulate glomerular sensitivity and sharpen MC tuning (Castillo et al., 1999; Panzanelli et al., 2007; D'Souza and Vijayaraghavan, 2012; Liu et al., 2015). Interestingly, pharmacological OB blockade of nAChRs impedes fine odor discrimination (Devore et al., 2014), which supports the idea that nAChRs are involved in MC tuning. While the exact mechanisms remain unclear, both $\mathrm{mAChRs}$ and $\mathrm{nAChRs}$ play a role in shaping GL processing and may impact $\mathrm{OB}$ output. Therefore, alterations of either subtype of AChRs could alter the tuning of OB output cells in a way that is functionally relevant to olfactory associative learning and contributes to either discrimination or generalization during fear expression. 


\section{Dopamine Receptors}

The OB of mice contains intrinsic dopaminergic neurons in the glomerular layer. These neurons represent the short axon cells (SACs; Halasz et al., 1977), a subpopulation of periglomerular cells that are also GABAergic. Unlike most brain regions where dopamine is secreted from terminals of projection neurons originating in dopaminergic brain nuclei, the OB only receives dopamine from this intrinsic source. Dopamine exerts its action through dopamine receptors. Of the five identified subtypes of dopamine receptors (D1-D5), D4 is the only transcript that has not yet been detected in the OB (Coronas et al., 1997). The $\mathrm{RT}^{2}$ Profiler PCR Array (Qiagen) provided primers to assess relative expression differences in D1, D2, and D5 (coded by the Drd1, Drd2, and Drd5 genes, respectively). Consistent with previous results, we were able to detect each the three transcripts in OB tissue. In situ hybridization and radioligand binding assays demonstrate that there are gene transcripts and functional D1-like receptors (D1 and D5) in most layers of the OB: GL, external plexiform layer (EPL), mitral cell layer (MCL), internal plexiform layer (IPL), and granule cell layer (GCL), but not in the olfactory nerve layer (ONL; Coronas 1997; Levey et al., 1993). In contrast, D2 receptors are expressed in the ONL and GL, and on mitral cells (Brunig et al., 1999; Coronas 1997; Davila et al., 2003). Interestingly, D2 receptors are expressed in OSNs in the ONL and OSN axon terminals in the GL, the GL compartments of mitral and tufted cell dendrites, and dendrites of the SACs (Gutierrez-Mecinas et al., 2005a). The D2 receptors are localized primarily to the presynaptic compartment of OSN excitatory synapses onto mitral/tufted cells (MTCs) and SACs, as well as the presynaptic specialization of MTC excitatory synapses onto SAC dendritic spines.

D1 and D2 receptors appear important for normal olfactory function as pharmacological systemic blockade of D1 receptors or genetic knockdown of D2 receptors decreases performance on olfactory tasks (Weldon et al., 1991; Tillerson et al., 2006). Both dopamine receptors putatively modulate neurotransmission between MTCs and interneurons. While activation of D1 receptors appears to reduce spontaneous excitatory postsynaptic potentials (EPSPs) in GL interneurons, it has no effect on evoked EPSPs. In contrast, activation of D2 receptors attenuates both spontaneous and evoked EPSPs in GL interneurons (Davila 2003). Agonism of D1 receptors reduces the GABAinduced currents in granule cells (Brunig 1999), which may indicate D1 signaling primarily affects the MTC-GC circuitry. D2 receptors are highly expressed in the GL and, in general, their activation appears to decrease excitatory neurotransmission. Given their localization within the GL, activation of D2 receptors results in decreased glutamate release from OSNs onto MTCs, decreased glutamate release from MTCs to SACs, and decreased inhibition from SACs onto MTCs ( Davila 2003; Vaaga et al., 2017; Guiterresz-Mecinas et al., 2005a). It is important to note that only a small percentage of D2 receptors found on SACs are linked to the plasma membrane (Guiterrez-Mecinas et al., 2005a) indicating modulation of the SAC-MTC circuit may be less likely through D2 signaling.

D1 and D2 receptors were downregulated 1.5 fold in Paired mice 4 hours after odor-shock pairing. This demonstrates that odor-shock pairing and olfactory fear learning 
alter the expression of D1 and D2 receptors in the OB. We find that gene expression of Drd1, which codes for the D1 receptor, is still downregulated 24 hours after olfactory fear conditioning, while expression of $\mathrm{Drd} 2$ has normalized to control levels. This decrease in expression may propagate to decreased protein translation and, therefore, fewer functional receptors, at least transiently in the case of D2 receptors. Given the presumed role of dopamine receptors enhancing OB inhibition, fewer receptors could result in overall increase in OB excitatory neurotransmission. There appear to be significantly more D2 receptors than D1 receptors in the OB, suggesting D2 may play a larger role in OB processing especially given that SACs, the sole source of OB dopamine, express D2 receptors. However, changes in the expression of either could significantly impact olfactory coding.

\section{GABA Receptors}

GABA is the primary inhibitory neurotransmitter in the developmentally mature central nervous system responsible for decreasing neuronal excitability. GABA receptors, which are ubiquitously expressed throughout the brain, can be divided into two major classes, $\mathrm{GABA}_{\mathrm{A}}$ and $\mathrm{GABA}_{\mathrm{B}}$ based on their transduction mechanisms. In the present study, we looked at olfactory fear conditioning-induced changes of GABA receptor gene expression. We found no changes in the gene expression of transcripts coding for $\mathrm{GABA}_{\mathrm{B}}$ receptors at either 4 or 24 hours post training, but found significant transcript regulation in the $\mathrm{GABA}_{\mathrm{A}}$ family of GABA receptors 4 hours after training. GABA $\mathrm{A}_{\mathrm{A}}$ receptors are multi-subunit ionotropic receptor complexes, comprising subunits from $\alpha$ (Gabra), $\beta$ (Gabrb), $\gamma$ (Gabrg), $\delta$ (Gabrd), $\varepsilon$ (Gabre), $\pi$ (Gabrp), $\theta$ (Gabrq), and $\rho$ (Gabrr) families (Enz and Cutting, 1998). Gene expression analysis reveals $\sim 1.5$-fold downregulation of Gabra2, Gabra4, Gabra5, Gabrb3, Gabrg2, Gabrg3, Gabrq, and Gabrrl in mice that experienced odor in the context of shock relative to controls. This would appear to suggest significant alterations in OB inhibition as a result of olfactory fear conditioning. Interestingly, $\mathrm{GABA}_{\mathrm{A}}$ receptors are known to be involved in both olfactory aversive learning (Okutani et al., 1999; Okutani et al., 2002) and olfactory discrimination (Mwilaria et al., 2008; Nunes and Kuner, 2015).

At least one report suggests that the $\alpha 5$ subunit is requisite for the formation of a functional $\mathrm{GABA}_{\mathrm{A}}$ receptor complex (Fritschy et al., 1997), indicating that downregulation of the Gabra5 transcript which codes for the $\alpha 5$ subunit may have significant impact on inhibition in the OB. In the OB, inhibitory, GABAergic periglomerular cells (PGCs) express both $\alpha 2$ and $\alpha 5$ subunits (Panzanelli et al., 2007), both of which are downregulated following olfactory fear conditioning. This indicates altered PGC inhibition onto the dendrites of output cells in the GL. The $\alpha 5$ subunit is also highly expressed in MCs, which could make them significantly less susceptible to the actions of GABA. Releasing MCs from inhibition could have a number of effects such as augmented noise-to signal or signal-to-noise, increased OB output, and long-term synaptic alterations (Pandipati et al., 2010; Zak et al., 2015). 
MCs display strong labeling to mRNA probes directed against transcripts for all three known $\beta$ subunits while GCs appear to only express the $\beta 3$ subunit (Laurie et al., 1992). The inclusions of $\beta$ subunits are also thought to be important for the formation of functional $\mathrm{GABA}_{\mathrm{A}}$ receptors (Homanics et al., 1997), which are known to play a role in lateral inhibition between MCs and GCs (Wellis and Kauer, 1993). Knockout mice lacking the $\beta 3$ subunit in both MCs and GCs demonstrate altered olfactory discrimination abilities (Nusser et al., 2001), which suggests that the nearly 2 -fold downregulation of this subunit after olfactory fear conditioning like alters olfactory processing. A more indepth understanding of the exact locus of the downregulation could give additional insight into the effect on olfaction.

The $\gamma 2$ subunit is expressed primarily in the lower half of the GL, possibly on TCs, and on MCs (Allen Mouse Brain Atlas, 2004); therefore, downregulation of the transcript suggests fewer GABA receptors on OB output cells. This represents further evidence of decreased inhibition on output cells as a result of olfactory fear conditioning. Less is known about the localization or direct effects of $\theta$ and $\rho$ subunits. Regardless, the overall trend in downregulation of $\mathrm{GABA}_{\mathrm{A}}$ receptors supports altered inhibitory tone in the $\mathrm{OB}$ and like increased $\mathrm{OB}$ output, which may contribute to expression of olfactory learning.

\section{Glutamate Receptors}

As the ubiquitous excitatory neurotransmitter in the central nervous system, glutamate neurotransmission is prominent in the OB for olfactory processing. Glutamate receptors, which are responsible for transmission of excitation from presynaptic to postsynaptic neurons, are divided into two major classes - ionotropic receptors, which are further subdivided into NMDA receptors (NMDARs), AMPA receptors (AMPARs), and kainate receptors (KARs), and metabotropic glutamate receptors (mGluRs), all of which are present in the OB. In our study, we detected significant gene regulation of some subunits of AMPARs, KARs, and mGluRs 4 hours following olfactory fear conditioning but detected no alterations in the gene expression of NMDA receptors. Interestingly, of the four glutamate receptor genes determined to be modulated by odor-shock pairing when compared to controls, only the Grik4 gene, which codes for a KAR, was upregulated. The other identified genes, Grml and Grm8, which code for different subunits of mGluRs, and Gria2, which codes for an AMPAR, were downregulated in mice receiving the odor-shock experience. By 24 hours, the gene expression of all glutamate receptors had normalized to control levels.

Glutamate receptors are present on excitatory OB output cells, excitatory interneurons, and inhibitory interneurons and the same receptor subtype is often, but not always, expressed in multiple cell types. In addition, specific cell types often express more than one glutamate receptor. For example, MCs express all known AMPARs and KARs, as well as a number of mGluRs (Duvoisin et al., 1995; Saugstad et al., 1997; Montague and Greer, 1999; Heinbockel et al., 2007). Glutamate receptors often complex with multiple subunits, such that each composition will result in unique pharmacological 
profiles (Nakanishi et al., 1994). Interestingly, MCs are the only OB cell type known to express KAR4 (Grik4), which was upregulated following odor-shock pairing. KAR4 is only a functional subtype when combined with other glutamate receptor subtypes (Montague and Greer, 1999; Blakemore et al., 2018). As it is not functional alone, its main role is putatively to alter the properties of glutamate receptor complexes and, as it is thought to only be expressed in MCs, it may be acting to modulate the activity of MCs specifically. KAR4 insertion appears to slow the deactivation of receptors (Mott et al., 2010; Fisher and Fisher 2014), suggesting gene expression upregulation of the receptor seen in our study following odor-shock pairing could lead to functional insertion of the KAR4 subtype into glutamate receptors on MCs, which could result in prolonged MC activity.

Four hours after odor-shock pairing, paired mice demonstrated downregulation of Gria2. Glutamate receptors that lack the AMPAR2 (Gria2) subunit are more likely to induce calcium influx (Lerea and McNamera 1993); therefore, downregulation could indicate more cells becoming calcium permeable. However, AMPARs are functionally expressed on MCs, GCs, and PGCs (Montague and Greer, 1999), making it difficult to determine exactly how gene regulation of a specific AMPAR might alter olfactory coding. One known role of AMPAR2 is that MCs excite GCs, in part, through AMPAR2 expressed on GC spines (Trombley and Westbrook 1990); therefore, it is possible that downregulation of AMPAR2 acts to increase MC excitability by releasing them from MC-driven, GC-mediated inhibition.

Similarly, the expression of the Grm 1 gene (mGluR1) was downregulated in paired mice. mGluR1 directly depolarizes MCs and increases firing, which would modulate the responsivity of MCs to OSN input (Heinbockel et al., 2004); however, mGluR1s are also present on a subpopulation of GCs (Heinbockel et al., 2007), suggesting changes in mGluR1 may alter MC excitability by either acting directly on MCs or by regulating the MC-GC circuit. In addition, mGluR1 increases the excitability of SACs (Jian et al., 2010); therefore, downregulation of the receptor could decrease SAC-mediated inhibition in the GL and exert a net gain of OB excitatory activity. Finally, mGluR8 is expressed strongly in the OB in both the MCL and GCL (Duvoisin et al., 1995; Saugstad et al., 1997) and appear to inhibit glutamate release at MC terminals, thereby regulating MC activity (Schoppa and Westbrook, 1997). Together, this suggests that altered gene expression of mGluRs after odor-shock pairing may ultimately act to increase excitatory OB output.

We demonstrate that 4 hours following olfactory fear conditioning, several glutamate receptors are downregulated and one, Grik4 a KAR, is upregulated. Previous reports suggest that upregulation and insertion of non-NMDA ionotropic glutamate receptors is associated with olfactory fear learning in neonatal rats (Cui et al. 2011; Modarresi et al. 2016); therefore, this may be a conserved mechanism in adults. The altered gene expression of glutamate receptor subtypes may change the pharmacological profiles of existing glutamate receptors or may represent changes in the number of functional receptors present in the OB. Given the role of glutamate receptors in neuronal excitability, either outcome would likely involve drastic changes to olfactory processing. 
To fully understand the extent of these outcomes, it would be necessary to identify where receptor subtype changes occur and the consequences on neuronal excitability as well as whether there is an overall difference in the total number of glutamate receptors between paired and control mice.

\section{Serotonin Receptors}

The OB receives serotonergic innervation from the raphe nuclei located in the brainstem (Levy et al., 1999) and serotonergic innervation is essential for olfactory associative learning (McLean et al., 1993; Price et al., 1998). While serotonergic fibers terminate in nearly every layer of the OB, the density of termination in the GL is 2-3 times that of any other layer (McLean and Shipley, 1987; Shipley and Ennis, 1996), suggesting that OB serotonin (5-HT) release primarily affects GL processing. 5-HT affects numerous sites in the GL. For example, 5-HT release directly excites TCs, which in turn excites SACs, which themselves can be directly excited by 5-HT. The activation of SACs will then inhibit both OSNs and MCs; however, MCs can also be excited by 5HT either directly or indirectly through other cells (Gaudry 2018). This means that 5-HT can have both an excitatory or inhibitory effect on MC processing (Kapoor et al., 2016) and could potentially do so in a glomerulus-specific fashion. Furthermore, 5-HT release could simultaneously inhibit some glomeruli while increasing excitation of others, which could alter glomerular receptive fields. 5-HT release also appears to attenuate responses of OSN, which decreases input to the GL (Petzold et al., 2009). This decreased input to the GL, the first site of odor processing in the brain, is likely to propagate to all the subsequent levels of olfactory processing. In addition, it appears that in the OB, 5-HT may affect odor processing by interacting with other transmitter systems (Bockaert et al., 1998; Yuan et al., 2003), thus providing another indirect mechanism by which 5-HT regulation can affect $\mathrm{OB}$ processing.

Even though 5-HT preferentially innervates the GL, the fibers do not target a single cell type but rather have widespread neuromodulatory influence through widely distributed receptors (Gaudry 2018). Many subtypes of 5-HT receptors are found in the rodent OB (Pazos et al., 1985a and b; McLean and Shipley 1987; Ruat et al.,1993; Shen et al., 1993; Martín-Cora and Pazos, 2004; Pompeiano et al., 1994; Lucaites et al., 2005; Koyama et al., 2017), but relatively little is known about their functional role in olfactory processing. In our gene expression analysis, we demonstrate that olfactory fear conditioning alters the transcripts of Htrlf, Htr2a,Htr2c, Htr3a, and Htr 7 at four hours post conditioning and that Htr 4 gene is regulated 24 hours post conditioning. In all cases, the identified genes that code for 5-HT receptors are downregulated with the exception of $H t r 2 c$, which codes for the 5-HT2c receptor and is more the 4-fold upregulated four hours post conditioning. Recent reports suggest that 5-HT signaling through 5-HT2c may depolarize PGCs and GCs (Hardy et al., 2005); however, it is unclear whether 5-HT2cmediated depolarization of PGCs has any functional effect on activity (Brill et al., 2016). One possibility is that signaling through 5-HT2c alters the inhibitory tone of the GL network in a glomerulus-specific fashion to increase MC synchrony, which can be important for learning (Kay and Beshel, 2010). In addition, agonizing $2 \mathrm{a} / 2 \mathrm{c}$ receptors 
supports olfactory learning (Price et al., 1998), demonstrating an important function for regulation of this receptor.

The gene expression of all other 5-HT receptors that were altered as a result of olfactory fear conditioning was downregulated. This is especially interesting given recent evidence that the main action of 5-HT release in the OB may be to attenuate OSN sensitivity (Petzold et al., 2009; Dugué and Mainen, 2009). However, others suggest that 5-HT-mediated increased GL inhibition may ultimately enhance sensitivity of GL processing (Brill et al., 2016; Kapoor et al., 2016). Further still, there is evidence that 5HT can induce spiking in MCs, which in turn excites GCs which themselves should inhibit MCs (Schmidt and Strowbridge, 2014). Therefore both the paucity of systematic study and inconsistencies in the reports of the effects of 5-HT on OB processing makes it difficult to interpret how alterations in transcripts for 5-HT receptors might impact functional olfactory perception. In addition, little is known about the specific contributions of different 5-HT receptors on olfactory processing; however, in other brain regions, activation of 5-HT2a appears to facilitate fear extinction (Zhang and Stackman, Jr. 2015) so it is feasible that downregulation of the receptor in the OB supports durability of olfactory learning. Furthermore, it seems that activation of 5-HT receptors in other systems may impair memory performance during recall (Meneses, 1998; Meneses et al., 1998a and b), so perhaps downregulating 5-HT receptors in the OB is somehow functionally relevant to olfactory memory. Future studies regarding the role of 5-HT and receptors on olfactory processing are still needed to fully understand how its regulation might impact olfactory associative learning.

\section{Somatostatin Receptors}

Somatostatin is a neuropeptide that is released from a number of neurons throughout the brain including the in the OB to modulate excitability and neuronal responses (Lepousez et al., 2010a and b; Liguz-Lecznar et al., 2016). Cellular markers for somatostatin frequently colocalize with markers for GABA (Panzanelli et al., 2007); however, they also colocalize with neuromodulatory projection neurons (Araneda et al., 1999). There are four identified somatostatin receptors (SSTRs) which are expressed throughout the OB (Kaupmann et al., 1993; Gutierrez-Mecinas et al., 2005b; Lepousez et al., 2010a; Nocera et al., 2019). Of those four, we assessed the olfactory fear conditioning-induced changes in gene expression of three, Sstr1, Sstr2, and Sstr4. Twenty-four hours after fear conditioning, both Sstrl and Sstr4 were down regulated $\sim 1.5$ - and $\sim 1.7$ fold, respectively. The Sstr 1 gene, which codes for SSTR1, the Sstr4 gene, which codes for SSTR4 are expressed in the GL, EPL, MCL, and IPL, but not the GCL (Bruno et al., 1993; Pérez and Hoyer, 1995; Debus et al., 2001; Allen Brain Atlas 2007; Nocera et al., 2019); however, very little is known about the role somatostatin plays in olfactory processing. Only a recent study demonstrates that genetic depletion of somatostatin or SSTR4 have no effect on olfactory-driven tasks, while deletion of SSTR2, which is expressed on all MC compartments, impacts olfactory detection and discrimination (Nocera et al., 2019). This would seem to suggest some functional role of somatostatin release and signaling through SSTRs in olfactory perception, indicating 
downregulation of the SSTR gene transcripts following olfactory fear conditioning might affect olfactory processing.

\section{Olfactory Fear Conditioning-Induced Alterations of Neuromodulation}

Within four hours of olfactory fear conditioning, mice experiencing odor paired with shocks exhibit significant changes in the transcripts of numerous genes that code for neurotransmitter receptors. While the exact role of many of the receptors and specific subunits has yet to be fully characterized, many of them appear to regulate inhibition in the OB. As most of the gene transcripts that were altered as a result of fear conditioning were downregulated relative to controls, this suggests olfactory fear conditioning may alter OB processing in a way that decreases overall inhibition. This decrease in inhibition likely releases OB input cell or principle output cells from inhibition, resulting in increased odor-evoked activation of excitatory cells, which is consistent with several recent reports demonstrating enhanced OB input or OB responses following olfactory fear conditioning (Fletcher, 2012; Kass et al., 2013; Ross and Fletcher, 2018). 


\section{CHAPTER 6. DISCUSSION}

A major symptom of anxiety disorders, such as panic disorder or post-traumatic stress disorder, is heightened fear responses. Fear is often learned through associative aversive conditioning, in which an organism learns to associate an initially meaningless and neutral stimulus with an innately fear inducing stimulus, which elicits a natural fear response. Following the temporal pairing of these stimuli, animals exhibit robust and stereotyped fear in response to the previously neutral stimulus (conditioned stimulus) that now predicts the aversive, fear-inducing event (Pavlov, 1927). This form of learning often leads individuals to exhibit strong fear responses to neutral, unlearned stimuli that are similar to the conditioned stimulus (CS), a process known as generalization. In most cases, the more a neutral stimulus perceptually resembles the learned CS, the greater the generalized response. One of the predominant theories regarding the cause of generalization is that during the learning processes, sensory processing changes in a way that increases the representational similarity between neutral stimuli and the CS, thus making them more difficult to perceptually discriminate (Lashley and Wade, 1946; Ghosh and Chattarji, 2015; Zaman et al., 2019).

The ability for the brain to flexibly adapt in response to changing environments is crucial to biological success. There is extensive evidence that the brain is adept at reorganizing sensory processing in response to change (Merzenich et al., 1983; Kelahan and Doetsch, 1984; Doetsch et al., 1988; Fregnac et al., 1988; Robertson and Irvine, 1989; Gonzalez-Lima and Agudo, 1990; Jenkins et al., 1990; Kaas et al., 1990; Garraghty and Kaas, 1991a,b; Fregnac et al., 1992; Merzenich and Jenkins, 1993; Mogilner et al., 1993; Pascual-Leone and Torres, 1993; Zarzecki et al., 1993; Valtcheva and Froemke, 2019). Experience and learning also represent change that requires altered neural processes. These changes take place in areas such as the hippocampus, amygdala, and association cortices (Gilmartin and McEchron, 2005; Holahan et al., 2006; Grossman et al., 2008; O'Neill and Schultz, 2018; Zhang and Li, 2018) but also in sensory regions (Letzkus et al., 2011; Nasir et al., 2013; Yuan and Harley, 2014; Quiroga, 2016; Ross and Fletcher 2018b).Yet, in terms of neuroplasticity following aversive learning, relatively little attention has been paid to sensory systems encoding the sensory stimuli that elicit fear.

While there are several reports of altered sensory coding following aversive learning that likely impacts behavior (Bakin and Weinberger, 1990; Edeline et al., 1993; Bakin et al., 1996; Siucinska and Kossut, 1996; Weinberger, 1998; Weinberger and Bakin, 1998; Weinberger, 2004; Barnes et al., 2011; Fletcher, 2012; Kass et al., 2013; Kass and McGann, 2017; Ross and Fletcher, 2018), most of the research focus is on the amygdala, which had led to a paucity of experiments testing the extent to which learning can be modulated by sensory processing and whether fear generalization might arise from failure of perceptual discrimination in sensory regions. Therefore, the aim of this dissertation was to understand fundamental properties of associative learning in terms of neural plasticity in early sensory regions but also to relate sensory plasticity to behavioral 
outputs and to test whether manipulating early sensory regions alone can impact strength of learning.

In order to better relate learning-induced neural changes to behavioral expression of fear, we performed all imaging experiments under awake, head-fixed conditions in the same mice that were behaviorally tested. Much of the original work investigating learning-induced alterations in sensory coding were performed under anesthetized conditions but evidence has emerged that there are significant differences between awake and anesthetized preparations that could greatly influence results(Castro-Alamancos, 2004; Kato et al., 2012; Cazakoff et al., 2014; Nunez-Parra et al., 2014; Sellers et al., 2015; Sabri and Arabzadeh, 2018). Importantly, this allowed for directly correlating neural responses to behavioral expression of fear to both the CS and neutral odors. In Chapter 2, we were able to replicate previous findings that olfactory fear learning enhances olfactory bulb (OB) glomerular responses in anesthetized mice but found that awake imaging reveals much stronger more robust changes in CS coding following fear learning. One interesting point is that passive odor exposure reduced odor-evoked glomerular responses in awake mice across days, which has now been reported in several studies. This differs from habituation, which is typically described as decreased responsivity that occurs within a session. Mice were subjected to three days of consecutive odor imaging and odor-evoked responses were significantly higher after fear conditioning than they were the day before but were also stronger than on the initial day of imaging. This seems to suggest that whatever the mechanism of learning-induced glomerular enhancements is, it is not simply an "undoing" of passive odor experience suppression of glomerular responses. Interestingly, no one has every directly studied the cause of passive experience-dependent response suppression and, while not particularly germane to the study of associative learning-induced response enhancements, it represents an interesting area for future investigation.

We additionally tested, in awake mice, behavioral generalization and glomerular responsivity to neutral odors, which were never paired with shock. By expanding the experimental design to assess neutral odors, we were able to determine that fear conditioning non-specifically enhances odor-evoked glomerular responses and that the effect of this is to increase the representational similarity between neutral odors and the CS, likely making them more perceptually similar and leading to failure of perceptual discrimination as the initial basis for fear generalization. Interestingly, the global glomerular enhancements are long lasting and are not caused by general fear states or changes in respiration, nor can they be blocked by inhibiting the amygdala after learning occurs. Together, we feel this represents a true change in early sensory processing in the $\mathrm{OB}$ rather than a purely top-down modulation of OB processing.

Using in vivo pharmacology, we were able to determine that there are actually two distinct mechanisms underlying the post-training glomerular enhancements. First, there appears to be a CS-specific enhancement of glomerular responses that does not require learning, as CS Responsive glomeruli are enhanced following odor-shock pairing even if behavioral learning is suppressed by inhibiting the amygdala during training. However, the second mechanism requires learning and supports the enhancement of glomeruli 
responding to the neutral odors. This demonstrates that the odor-shock pairing can alter $\mathrm{CS}$ coding in the $\mathrm{OB}$ even in the absence of learning but cannot alter coding of neutral stimuli under the same circumstances, further strengthening the theory that a relationship exists between altered sensory processing and behavioral fear generalization.

Previous work indicates that systemically antagonizing acetylcholine (ACh) receptors (AChRs) with scopolamine (SCOP), a muscarinic AChR (mAChR) antagonist, can change generalization across odorants following olfactory fear conditioning (Pavesi et al., 2012); however, it remained unknown whether ACh was required in the OB for fear learning to occur or whether cholinergic signaling has any effect on learning-induced plasticity. In Chapter 3 we determined that cholinergic neurotransmission, specifically via $\mathrm{m} 1 \mathrm{mAChRs}$ is required during odor-shock pairing in order for mice to acquire olfactory fear. Interestingly, mAChRs are not required during expression of previously learned olfactory fear. ACh is also capable of enhancing the strength of olfactory fear learning. By optogenetically stimulating release of ACh directly into the olfactory bulb during odor-shock pairing, we can increase the amount of freezing mice exhibit to the CS. Finally, systemic administration of a global $\mathrm{mAChR}$ antagonist during fear conditioning blocks fear-learning induced glomerular enhancements. While this experiment does not directly demonstrate that $\mathrm{ACh}$ is required in the $\mathrm{OB}$ during learning in order for $\mathrm{OB}$ neuroplasticity associated with odor-shock pairing to occur, it does expose an important role of ACh in learning-induced plasticity.

Most importantly, systemic administration of SCOP during conditioning prevents learning similarly to inhibiting the amygdala during conditioning; however, inhibiting the amygdala does not affect CS-specific glomerular enhancements, whereas blocking mAChRs with SCOP does. Together, this suggests ACh is crucial to CS-specific enhancements. We did not look at the effects of ACh modulation on generalization; however, this could provide interesting insights in the future. Perhaps opto/chemogeneticaly enhancing $\mathrm{OB}$ ACh during odor-shock pairing would further increase glomerular responses. Alternatively, increased OB ACh may make learning more specific, in which case it would be interesting to understand how glomerular responses relate. In Chapter 2 we find evidence that perhaps glomerular response enhancements can separately serve to increase or decrease perceptual similarity of odors based on whether the enhancements are seen in glomeruli that are CS Responsive or glomeruli that are Non-CS Responsive.

Glomeruli encompass several cell types, some of which are intrinsic to the OB and others which transmit information to other processing areas. Therefore, while it was clear from Chapter 2 that the mixed excitatory cell population responses were enhanced following olfactory fear learning, it was unclear the extent to which similar coding alterations exist at the somatic level of output cells that ultimately project sensory information out of the OB. In Chapter 4 we addressed this by using $2 \mathrm{P}$ imaging to specifically measure changes in two populations of OB output cells, superficial tufted cells (STCs) and mitral cells (MCs). Again, we included analysis of learning-induced changes of CS processing as well as neutral odor processing to understand how these specific output cell populations relate to behavioral fear generalization. 
The STCs and MCs are distinct populations of output cells both anatomically and functionally (Nagayama et al., 2004; Griff et al., 2008a,b; Nagayama et al., 2010; Kikuta et al., 2013; Vaaga and Westbrook, 2016; Tavakoli et al., 2018), warranting individual analysis of both. STC responses are tightly locked to odor presentations, with minimal spontaneous activity, inhibition, or temporal lag relative to odor onset. In Chapter 4 we were able to replicate a number of the basic findings from Chapter 2. For example, passive odor exposure results in decreased odor-evoked responses both at the glomerular level and at the somatic level in STCs. Similarly, olfactory fear learning enhances STC responses evoked by CS presentations as well as those evoked by neutral odors, as was seen in glomeruli.

There is some evidence that STC enhancements are CS-specific, wherein STCs that respond to neutral odors but also to the CS are more enhanced than those that respond only to neutral odors. This may represent something similar to the CS-specific mechanism reported in Chapter 2; however, an additional study in which learning is blocked would be required to address whether these seemingly CS-specific STC enhancements occur independent of learning, as was the case for CS-specific glomerular enhancements. Alternatively, there are other populations of intrinsic excitatory OB cells that may support the CS-specific, learning-independent glomerular enhancements we observed. The primary role of the enhanced STC responses appears to be increasing the behavioral salience of incoming sensory information, as there is no change in the odor identity coding of these cells. Behavioral assays, such as odor sensitivity tasks or olfactory investigation tasks, are needed to validate the idea that enhanced STC responses lead to increased behavioral salience of odors.

MCs, unlike STCs, have several different response profiles. There are MCs that are excited by odors while others are inhibited; some MCs have transient odor responses, while others are prolonged. There is also significant temporal variation in onset of MC response relative to the time of odor onset. Remarkably, the same MC may respond to different odors with completely different response profiles. We employed a k-means cluster analysis to help categorize the MCs based on their odor-evoked response profile. While MCs appear to cluster into 5 distinct profiles, the most simplistic clustering splits $\mathrm{MC}$ responses into a net excitatory or net inhibitory type. Again, passive odor exposure decreases the odor-evoked responses, but seemingly only in excitatory MCs, whereas inhibitory MCs don't change on average after odor experience. There was also little to no change in the inhibitory MCs after olfactory fear conditioning but much stronger responses in the excitatory MCs. The MC effects, at least in the excitatory MCs, resemble the olfactory fear learning-induced effects in both glomeruli and STCs, suggesting that MCs may also code for increased salience. Again, behavioral experiments confirming this hypothesis are warranted.

In addition to enhanced odor-evoked responses, analysis of MCs revealed increased ensemble correlations between the CS and neutral odors after olfactory fear conditioning. This represents an important distinction between STCs and MCs. We also identified increased representational correlations in glomerular imaging, suggesting MCs are responsible for this effect. This increased ensemble similarity makes the MC coding 
of neutral odors more similar to the coding of the CS, which, when propagated to other processing areas, should serve to make neutral odors more perceptually similar to the CS. We believe that this likely makes the odors more difficult to discriminate and that this failure to discriminate ultimately leads to behavioral fear generalization. If true, this suggests that fear-learning induced changes in MC coding constitute the initial neural basis for fear generalization and that suppressing these changes should prevent generalization. While targeted manipulation of MC coding is not currently technically possible, future advances may enable directly testing this hypothesis.

We clustered MCs into 2 clusters, which appeared to differentiate MC responses based primarily on the net amplitude of the evoked response. However, MCs have dynamic temporal responses in addition to varied response amplitudes. We have also found that MCs exhibit highly variable response amplitudes throughout the imaging trial and odor presentation. For example, some MCs have a small inhibitory event during the odor followed by a large excitatory response after and may be clustered as a net excitatory response. Therefore by clustering MCs into only two clusters, we decrease the dimensionality $\mathrm{MC}$ odor responses. In the future, the $\mathrm{MC}$ data will be reclustered into the five distinct clusters, which we feel better represents the rich temporal and amplitude signatures exhibited by MCs. The reclustered data will then be analyzed to determine whether there is a more nuanced effect of learning on MCs. We have also identified a significant number of cells that switch clusters after olfactory fear conditioning, and these may be highly important. One basic idea would be to identify neutral odor-cell pairs that switch clusters and determine whether they switch clusters to more closely align with the corresponding CS-cell pair. This seems especially important considering a number of the cells switch from inhibitory type clusters to more excitatory type clusters and vice versa, which would represent robust reorganization of olfactory coding in MCs.

The question of how olfactory fear conditioning alters the OB to enhance glomerular (specifically STC and MC) odor-evoked responses and change odor-identity coding remains. Based on the results obtained in Chapter 3 and due to the fact that the OB receives significant neuromodulatory innervation (Fletcher and Chen 2010), we performed an unbiased gene expression analysis of neurotransmitter receptors. In Chapter 5, we extracted the OBs at 4- and 24-hours following olfactory fear conditioning or control conditioning for analysis of over 80 neurotransmitter receptor genes. Most of the results point to downregulation of inhibitory receptors. This could explain the enhanced responses as well as the increased representational similarity by reducing inhibition onto excitatory cells and effectively broadening receptive fields or changing cellular sensitivity. One interesting future direction would be to employ electrophysiological techniques to determine whether there is reduced inhibition onto excitatory output cells or reduced presynaptic inhibition onto the olfactory sensory neurons (OSNs) that excite output cells. Reduced inhibition, likely via the MC-granule cell circuit, and reduced presynaptic inhibition, likely via short axon cells onto OSNs, represents two of the most likely targets given the downregulation of GABA and dopamine receptors, respectively. The results of the gene expression analysis also indicated significant upregulation of a serotonin receptor, 5-HT2c, four hours after olfactory fear conditioning. One recent paper demonstrates that mice lacking the serotonin transporter more readily learn discriminant 
fear conditioning (Lima et al., 2019). This could suggest that the upregulation of the receptor might play a role in olfactory fear generalization. Ultimately, it will be necessary to confirm these initial findings at the protein level and to use techniques such as in situ or immunohistochemistry to identify specific cell types in which these receptors are changed.

While this work only encompasses one model system and a single animal model, we feel that the basic principles we report extend beyond these limitations. Other studies also demonstrate changes in a variety of sensory systems and animal models as a result of fear learning (Bakin and Weinberger, 1990; Scheich and Zuschratter, 1995; Bakin et al., 1996; Siucinska and Kossut, 1996; Weinberger and Bakin, 1998; Weinberger, 2004). The presented data likely also extend beyond fear learning to any learning that results in generalization, whether that be aversive or appetitive, such as an operant task where animals are taught to treat different sensory stimuli as the same because they are paired with the same reward. In addition, our results may provide important insights for mechanisms of discriminant learning. Previous work suggests behavioral discrimination correlates with decorrelated representations of sensory stimuli in cortex (Chen et al., 2001). We propose that the switch basis for behavioral discrimination or generalization is a function of sensory stimulus correlation, where decorrelated responses lead to discrimination and more correlated responses lead to generalization. Under this assumption, understanding one side of the behavior and underlying mechanisms will be valuable to investigating the other.

One method of testing this hypothesis would be to compare glomerular and celltype specific changes between the classical fear learning paradigm we have already employed and a discriminative paradigm. Discriminant conditioning uses a CS+, a stimulus paired with shock, and a CS-, a stimulus never paired with shock, in an attempt to teach animals to fear only the CS+ and not the CS-. In this case there should be no behavioral generalization. Based on the interpretation of our results then, we would expect either to see only CS-specific enhancements or decreased ensemble correlations that would make odors more easily discriminable. It would be especially interesting to image $\mathrm{OB}$ responses during a discriminant olfactory fear conditioning paradigm. It is difficult for mice to learn specific fear through discriminant paradigms, whereas mice can readily learn a classical olfactory fear, and will behaviorally generalize, in as little as one pairing. This could suggest that the default process is fear generalization and that discrimination takes extensive learning to effectively suppress the tendency to generalize. Imaging during the learning paradigm might provide insights into this if odor-shock pairing immediately begins to potentiate all odor-evoked responses but the change becomes more specific to the CS+ as more CS- trails accumulate.

We believe that our data demonstrate fear learning alters early sensory coding in ways that support both increased salience of incoming sensory information and behavioral generalization by making responses to neutral stimuli more similar to the CS. This increased similarity should make odors perceptually harder to distinguish, leading to a failure of discrimination, and ultimately fear generalization. Most of the work involving mechanisms of fear learning and generalization centers around the amygdala; however, 
we were able to demonstrate that, while it blocks expression of learned fear, inhibiting the amygdala does not alter the learning-induced enhancement of glomerular responses. This unequivocally demonstrates that there are important neural changes due to fear learning outside of the amygdala that likely contribute to behavioral responses and could be relevant to the future study of aberrant fear learning and fear generalization. 


\section{LIST OF REFERENCES}

Akerboom J, Chen T, Wardill TJ, Marvin JS, Mutlu S, Calderon NC, Esposti F, Borghuis BG, Sun XR, Gordus A, Orger MB, Portugues R, Engert F, Macklin JJ, Filosa A, Aggarwal A, Kerr RA, Takagi R, Kracun S, Shigetomi E, Khakh BS, Baier H, Lagnado L, Wang SSH, Bargmann CI, Kimmel BE, Jayaraman V, Svoboda K, Kim DS, Schreiter ER, Looger LL (2012) Optimization of a GCaMP calcium indicator for neural activity imaging J Neurosci 32:13819-13840 doi:10.1523/JNEUROSCI.2601-12.2012

Alberini CM, Kandel ER (2014) The regulation of transcription in memory consolidation. Cold Spring Harb Perspect Biol 7:a021741.

Alkondon M, Rocha ES, Maelicke A, Albuquerque EX (1996) Diversity of nicotinic acetylcholine receptors in rat brain. V. alpha-Bungarotoxin-sensitive nicotinic receptors in olfactory bulb neurons and presynaptic modulation of glutamate release. J Pharmacol Exp Ther 278:1460-1471.

Allen Brain Atlas (2004) Allen Mouse Brain Atlas. Inc.

Almli CR, Henault MA, Velozo CA, Morgane PJ (1985) Ontogeny of electrical activity of main olfactory bulb in freely-moving normal and malnourished rats Brain Res 350:1-11

Alonso M, Viollet C, Gabellec MM, Meas-Yedid V, Olivo-Marin JC, Lledo PM (2006) Olfactory discrimination learning increases the survival of adult-born neurons in the olfactory bulb J Neurosci 26:10508-10513 doi:10.1523/JNEUROSCI.263306.2006

Amtul Z, Atta Ur R (2015) Neural plasticity and memory: molecular mechanism Rev Neurosci 26:253-268 doi:10.1515/revneuro-2014-0075

Anagnostaras SG, Maren S, Sage JR, Goodrich S, Fanselow MS (1999) Scopolamine and Pavlovian fear conditioning in rats: dose-effect analysis. Neuropsychopharmacology 21:731-744.

Araneda S, Gysling K, Calas A (1999) Raphe serotonergic neurons projecting to the olfactory bulb contain galanin or somatostatin but not neurotensin. Brain Res Bull 49:209-214.

Arenas A, Giurfa M, Sandoz JC, Hourcade B, Devaud JM, Farina WM (2012) Early olfactory experience induces structural changes in the primary olfactory center of an insect brain. Eur J Neurosci 35:682-690.

Bakin JS, South DA, Weinberger NM (1996). Induction of receptive field plasticity in the auditory cortex of the guinea pig during instrumental avoidance conditioning. Behav Neurosci 110(5), 905-913.

Bakin JS, Weinberger NM (1990) Classical conditioning induces CS-specific receptive field plasticity in the auditory cortex of the guinea pig. Brain Res 536:271-286.

Barkai E, Bergman RE, Horwitz G, Hasselmo ME (1994) Modulation of associative memory function in a biophysical simulation of rat piriform cortex $\mathrm{J}$ Neurophysiol 72:659-677 doi:10.1152/jn.1994.72.2.659

Barkai E, Hasselmo MH (1997) Acetylcholine and associative memory in the piriform cortex Mol Neurobiol 15:17-29 doi:10.1007/BF02740613 
Barnes DC, Chapuis J, Chaudhury D, Wilson DA (2011) Odor fear conditioning modifies piriform cortex local field potentials both during conditioning and during postconditioning sleep PLoS One 6:e18130 doi:10.1371/journal.pone.0018130

Bauer EP (2015) Serotonin in fear conditioning processes. Behav Brain Res 277:68-77.

Bendahmane M, Ogg MC, Ennis M, Fletcher ML (2016) Increased olfactory bulb acetylcholine bi-directionally modulates glomerular odor sensitivity. Sci Rep 6:25808.

Blanchard RJ, Blanchard DC (1969) Passive and active reactions to fear-eliciting stimuli. J Comp Physiol Psychol 68:129-135.

Blauvelt DG, Sato TF, Wienisch M, Knopfel T, Murthy VN (2013) Distinct spatiotemporal activity in principal neurons of the mouse olfactory bulb in anesthetized and awake states. Front Neural Circuits 7:46.

Blum AL, Li W, Cressy M, Dubnau J (2009) Short- and long-term memory in Drosophila require cAMP signaling in distinct neuron types. Curr Biol 19:1341-1350.

Bockaert J, Claeysen S, Sebben M, Dumuis A (1998) 5-HT4 receptors: gene, transduction and effects on olfactory memory. Ann N Y Acad Sci 861:1-15.

Bonner TI, Young AC, Brann MR, Buckley NJ (1988) Cloning and expression of the human and rat $\mathrm{m} 5$ muscarinic acetylcholine receptor genes. Neuron 1:403-410.

Bower AJ (1990) Plasticity in the adult and neonatal central nervous system Br J Neurosurg 4:253-264

Boyd AM, Kato HK, Komiyama T, Isaacson JS (2015) Broadcasting of cortical activity to the olfactory bulb. Cell Rep 10:1032-1039.

Bozza T, McGann JP, Mombaerts P, Wachowiak M (2004) In vivo imaging of neuronal activity by targeted expression of a genetically encoded probe in the mouse Neuron 42:9-21

Brennan PA, Schellinck HM, de la Riva C, Kendrick KM, Keverne EB (1998) Changes in neurotransmitter release in the main olfactory bulb following an olfactory conditioning procedure in mice Neuroscience 87:583-590

Bressler SL (1988) Changes in electrical activity of rabbit olfactory bulb and cortex to conditioned odor stimulation Behav Neurosci 102:740-747

Brill J, Shao Z, Puche AC, Wachowiak M, Shipley MT (2016) Serotonin increases synaptic activity in olfactory bulb glomeruli. J Neurophysiol 115:1208-1219.

Broersen LM (2000) Attentional processes and learning and memory in rats: the prefrontal cortex and hippocampus compared Prog Brain Res 126:79-94 doi:10.1016/S0079-6123(00)26008-1

Brosh I, Rosenblum K, Barkai E (2006) Learning-induced reversal of the effect of noradrenalin on the postburst AHP J Neurophysiol 96:1728-1733 doi: $10.1152 /$ jn. 00376.2006

Broussard GJ, Liang R, Tian L (2014) Monitoring activity in neural circuits with genetically encoded indicators Front Mol Neurosci 7:97 doi:10.3389/fnmol.2014.00097

Brunert D, Tsuno Y, Rothermel M, Shipley MT, Wachowiak M (2016) Cell-TypeSpecific Modulation of Sensory Responses in Olfactory Bulb Circuits by Serotonergic Projections from the Raphe Nuclei. J Neurosci 36:6820-6835. 
Brunig I, Sommer M, Hatt H, Bormann J (1999) Dopamine receptor subtypes modulate olfactory bulb gamma-aminobutyric acid type A receptors. Proc Natl Acad Sci U S A 96:2456-2460.

Bruno JF, Xu Y, Song J, Berelowitz M (1993) Tissue distribution of somatostatin receptor subtype messenger ribonucleic acid in the rat. Endocrinology 133:25612567.

Buck L, Axel R (1991) A novel multigene family may encode odorant receptors: a molecular basis for odor recognition Cell 65:175-187

Buonviso N, Chaput M (2000) Olfactory experience decreases responsiveness of the olfactory bulb in the adult rat. Neuroscience 95:325-332.

Buonviso N, Gervais R, Chalansonnet M, Chaput M (1998) Short-lasting exposure to one odour decreases general reactivity in the olfactory bulb of adult rats. Eur J Neurosci 10:2472-2475.

Burton, S.D. (2017). Inhibitory circuits of the mammalian main olfactory bulb. J Neurophysiol 118(4), 2034-2051. doi: 10.1152/jn.00109.2017.

Cahill SP, Foa EB (2007) In: Handbook of PTSD: Science and Practice (Friedman MJ, Keane TM, Resick PA, eds), pp 55-77. New York: Guilford.

Carasatorre M, Ramirez-Amaya V (2013) Network, cellular, and molecular mechanisms underlying long-term memory formation Curr Top Behav Neurosci 15:73-115 doi:10.1007/7854_2012_229

Carleton A, Rochefort C, Morante-Oria J, Desmaisons D, Vincent JD, Gheusi G, Lledo PM (2002) Making scents of olfactory neurogenesis J Physiol Paris 96:115-122

Case DT, Burton SD, Gedeon JY, Williams SG, Urban NN, Seal RP (2017) Layer- and cell type-selective co-transmission by a basal forebrain cholinergic projection to the olfactory bulb. Nat Commun 8:652.

Castillo PE, Carleton A, Vincent JD, Lledo PM (1999) Multiple and opposing roles of cholinergic transmission in the main olfactory bulb. J Neurosci 19:9180-9191.

Castro-Alamancos, M.A. (2004). Absence of rapid sensory adaptation in neocortex during information processing states. Neuron 41(3), 455-464.

Cavallaro S, D'Agata V, Manickam P, Dufour F, Alkon DL (2002) Memory-specific temporal profiles of gene expression in the hippocampus. Proc Natl Acad Sci U S A 99:16279-16284.

Cazakoff BN, Lau BY, Crump KL, Demmer HS, Shea S.D. (2014). Broadly tuned and respiration-independent inhibition in the olfactory bulb of awake mice. Nat Neurosci 17(4), 569-576. doi: 10.1038/nn.3669.

Chabaud P, Ravel N, Wilson DA, Mouly AM, Vigouroux M, Farget V, Gervais R (2000) Exposure to behaviourally relevant odour reveals differential characteristics in rat central olfactory pathways as studied through oscillatory activities Chem Senses 25:561-573

Chan W, Singh S, Keshav T, Dewan R, Eberly C, Maurer R, Nunez-Parra A, Araneda RC. (2017) Mice Lacking M1 and M3 Muscarinic Acetylcholine Receptors Have Impaired Odor Discrimination and Learning Front Synaptic Neurosci 9:4 doi:10.3389/fnsyn.2017.00004

Chapuis J, Wilson DA (2013) Cholinergic modulation of olfactory pattern separation. Neurosci Lett 545:50-53. 
Chaudhury D, Escanilla O, Linster C (2009) Bulbar acetylcholine enhances neural and perceptual odor discrimination J Neurosci 29:52-60

doi:10.1523/JNEUROSCI.4036-08.2009

Cheetham CE, Park U, Belluscio L (2016) Rapid and continuous activity-dependent plasticity of olfactory sensory input Nat Commun 7:10729

doi: $10.1038 /$ ncomms 10729

Chen CF, Barnes DC, Wilson DA (2011) Generalized vs. stimulus-specific learned fear differentially modifies stimulus encoding in primary sensory cortex of awake rats J Neurophysiol 106:3136-3144 doi:10.1152/jn.00721.2011

Chen JY, Marachlian E, Assisi C, Huerta R, Smith BH, Locatelli F, Bazhenov M (2015) Learning modifies odor mixture processing to improve detection of relevant components. J Neurosci 35:179-197.

Chen Q, Cichon J, Wang W, Qiu L, Lee SJ, Campbell NR, Destefino N, Goard MJ, Fu Z, Yasuda R, Looger LL, Arenkiel BR, Gan WB, Feng G (2012) Imaging neural activity using Thyl-GCaMP transgenic mice. Neuron 76:297-308.

Chettih SN, McDougle SD, Ruffolo LI, Medina JF (2011) Adaptive timing of motor output in the mouse: the role of movement oscillations in eyelid conditioning. Front Integr Neurosci 5:72.

Choi GB, Stettler DD, Kallman BR, Bhaskar ST, Fleischmann A, Axel R (2011) Driving opposing behaviors with ensembles of piriform neurons Cell 146:1004-1015 doi:10.1016/j.cell.2011.07.041

Chu MW, Li WL, Komiyama T. (2016). Balancing the Robustness and Efficiency of Odor Representations during Learning. Neuron 92(1), 174-186. doi: 10.1016/j.neuron.2016.09.004.

Coopersmith R, Lee S, Leon M (1986) Olfactory bulb responses after odor aversion learning by young rats Brain Res 389:271-277

Coronas V, Srivastava LK, Liang JJ, Jourdan F, Moyse E (1997) Identification and localization of dopamine receptor subtypes in rat olfactory mucosa and bulb: a combined in situ hybridization and ligand binding radioautographic approach. $\mathrm{J}$ Chem Neuroanat 12:243-257.

Cowansage KK, LeDoux JE, Monfils MH (2010) Brain-derived neurotrophic factor: a dynamic gatekeeper of neural plasticity Curr Mol Pharmacol 3:12-29

Crespo C, Blasco-Ibanez JM, Brinon JG, Alonso JR, Dominguez MI, Martinez-Guijarro FJ (2000) Subcellular localization of $\mathrm{m} 2$ muscarinic receptors in GABAergic interneurons of the olfactory bulb. Eur J Neurosci 12:3963-3974.

Csardi G, Franks A, Choi DS, Airoldi EM, Drummond DA (2015) Accounting for experimental noise reveals that mRNA levels, amplified by post-transcriptional processes, largely determine steady-state protein levels in yeast. PLoS Genet 11:e1005206.

Cui W, Smith A, Darby-King A, Harley CW, McLean JH (2007) A temporal-specific and transient cAMP increase characterizes odorant classical conditioning. Learn Mem 14:126-133.

Cui W, Darby-King A, Grimes MT, Howland JG, Wang YT, McLean JH, Harley CW (2011) Odor preference learning and memory modify GluA1 phosphorylation and GluA1 distribution in the neonate rat olfactory bulb: testing the AMPA receptor 
hypothesis in an appetitive learning model Learn Mem 18:283-291

doi:10.1101/lm.1987711

Cullinan WE, Herman JP, Battaglia DF, Akil H, Watson SJ (1995) Pattern and time course of immediate early gene expression in rat brain following acute stress. Neuroscience 64:477-505.

Curzon P, Rustay NR, Browman KE (2009) Cued and Contextual Fear Conditioning for Rodents. In: nd, Buccafusco JJ (eds) Methods of Behavior Analysis in Neuroscience. Frontiers in Neuroscience. Boca Raton (FL),

D'Agata V, Cavallaro S (2003) Hippocampal gene expression profiles in passive avoidance conditioning. Eur J Neurosci 18:2835-2841.

D'Souza RD, Vijayaraghavan S (2012) Nicotinic receptor-mediated filtering of mitral cell responses to olfactory nerve inputs involves the alpha3beta4 subtype. J Neurosci 32:3261-3266.

Davila NG, Blakemore LJ, Trombley PQ (2003) Dopamine modulates synaptic transmission between rat olfactory bulb neurons in culture. J Neurophysiol 90:395-404.

de Almeida L, Idiart M, Linster C (2013) A model of cholinergic modulation in olfactory bulb and piriform cortex. J Neurophysiol 109:1360-1377.

Debus N, Dutour A, Vuaroqueaux V, Oliver C, Ouafik L (2001) The ovine somatostatin receptor subtype 1 (osst1): partial cloning and tissue distribution. Domest Anim Endocrinol 21:73-84.

Devore S, Manella LC, Linster C (2012) Blocking muscarinic receptors in the olfactory bulb impairs performance on an olfactory short-term memory task Front Behav Neurosci 6:59 doi:10.3389/fnbeh.2012.00059

Devore S, de Almeida L, Linster C (2014) Distinct roles of bulbar muscarinic and nicotinic receptors in olfactory discrimination learning. J Neurosci 34:1124411260.

Di Prisco GV, Freeman WJ (1985). Odor-related bulbar EEG spatial pattern analysis during appetitive conditioning in rabbits. Behav Neurosci 99(5), 964-978.

Dietz SB, Markopoulos F, Murthy VN (2011) Postnatal development of dendrodendritic inhibition in the Mammalian olfactory bulb Front Cell Neurosci 5:10 doi:10.3389/fncel.2011.00010

Dittman AH, Weber JP, Hinds TR, Choi EJ, Migeon JC, Nathanson NM, Storm DR (1994) A novel mechanism for coupling of m4 muscarinic acetylcholine receptors to calmodulin-sensitive adenylyl cyclases: crossover from $\mathrm{G}$ protein-coupled inhibition to stimulation. Biochemistry 33:943-951.

Doetsch GS, Standage GP, Johnston KW, Lin CS (1988). Intracortical connections of two functional subdivisions of the somatosensory forepaw cerebral cortex of the raccoon. J Neurosci 8(6), 1887-1900.

Doty RL, Bagla R, Kim N (1999) Physostigmine enhances performance on an odor mixture discrimination test Physiol Behav 65:801-804

Doty RL, Bagla R, Misra R, Mueller E, Kerr KL (2003) No influence of scopolamine hydrobromide on odor detection performance of rats. Chem Senses 28:761-765.

Dunsmoor JE, Paz R (2015). Fear Generalization and Anxiety: Behavioral and Neural Mechanisms. Biol Psychiatry 78(5), 336-343. doi:

10.1016/j.biopsych.2015.04.010. 
Duvoisin RM, Zhang C, Ramonell K (1995) A novel metabotropic glutamate receptor expressed in the retina and olfactory bulb. J Neurosci 15:3075-3083.

Edeline JM, Pham P, Weinberger NM (1993) Rapid development of learning-induced receptive field plasticity in the auditory cortex. Behav Neurosci 107:539-551.

Edfors F, Danielsson F, Hallstrom BM, Kall L, Lundberg E, Ponten F, Forsstrom B, Uhlen M (2016) Gene-specific correlation of RNA and protein levels in human cells and tissues. Mol Syst Biol 12:883.

Elaagouby A, Ravel N, Gervais R (1991) Cholinergic modulation of excitability in the rat olfactory bulb: effect of local application of cholinergic agents on evoked field potentials. Neuroscience 45:653-662.

Faber T, Joerges J, Menzel R (1999) Associative learning modifies neural representations of odors in the insect brain Nat Neurosci 2:74-78 doi:10.1038/4576

Fanselow MS (1980) Conditioned and unconditional components of post-shock freezing. Pavlov J Biol Sci 15:177-182.

Fisher MT, Fisher JL (2014) Contributions of different kainate receptor subunits to the properties of recombinant homomeric and heteromeric receptors. Neuroscience 278:70-80.

Fletcher ML (2011) Analytical processing of binary mixture information by olfactory bulb glomeruli. PLoS One 6:e29360.

Fletcher ML (2012) Olfactory aversive conditioning alters olfactory bulb mitral/tufted cell glomerular odor responses Front Syst Neurosci 6:16 doi:10.3389/fnsys.2012.00016

Fletcher ML, Chen WR (2010) Neural correlates of olfactory learning: Critical role of centrifugal neuromodulation Learn Mem 17:561-570 doi:10.1101/lm.941510

Fletcher ML, Masurkar AV, Xing J, Imamura F, Xiong W, Nagayama S, Mutoh H, Greer CA, Knofel T, Chen WR (2009). Optical imaging of postsynaptic odor representation in the glomerular layer of the mouse olfactory bulb. J Neurophysiol 102(2), 817-830. doi: 10.1152/jn.00020.2009.

Fletcher ML, Smith AM, Best AR, Wilson DA (2005) High-frequency oscillations are not necessary for simple olfactory discriminations in young rats $\mathrm{J}$ Neurosci 25:792-798 doi:10.1523/JNEUROSCI.4673-04.2005

Fletcher ML, Wilson DA (2003) Olfactory bulb mitral-tufted cell plasticity: odorantspecific tuning reflects previous odorant exposure. J Neurosci 23:6946-6955.

Foehring RC, Lorenzon NM (1999) Neuromodulation, development and synaptic plasticity Can J Exp Psychol 53:45-61

Freeman WJ, Schneider W (1982) Changes in spatial patterns of rabbit olfactory EEG with conditioning to odors Psychophysiology 19:44-56

Fregnac Y, Shulz D, Thorpe S, Bienenstock E (1988). A cellular analogue of visual cortical plasticity. Nature 333(6171), 367-370. doi: 10.1038/333367a0.

Fregnac Y, Shulz D, Thorpe S, Bienenstock E (1992). Cellular analogs of visual cortical epigenesis. I. Plasticity of orientation selectivity. J Neurosci 12(4), 1280-1300.

Friedrich RW, Stopfer M (2001). Recent dynamics in olfactory population coding. Curr Opin Neurobiol 11(4), 468-474.

Fritschy JM, Benke D, Johnson DK, Mohler H, Rudolph U (1997) GABAA-receptor alpha-subunit is an essential prerequisite for receptor formation in vivo. Neuroscience 81:1043-1053. 
Ganesh A, Bogdanowicz W, Haupt M, Marimuthu G, Rajan KE (2010) Role of olfactory bulb serotonin in olfactory learning in the greater short-nosed fruit bat, Cynopterus sphinx (Chiroptera: Pteropodidae) Brain Res 1352:108-117 doi:10.1016/j.brainres.2010.06.058

Garraghty PE, Kaas JH (1991a). Functional reorganization in adult monkey thalamus after peripheral nerve injury. Neuroreport 2(12), 747-750.

Garraghty PE, Kaas JH (1991b). Large-scale functional reorganization in adult monkey cortex after peripheral nerve injury. Proc Natl Acad Sci U S A 88(16), 6976-6980.

Gaudry Q (2018) Serotonergic Modulation of Olfaction in Rodents and Insects. Yale J Biol Med 91:23-32.

Gdalyahu A, Tring E, Polack PO, Gruver R, Golshani P, Fanselow MS, Silva AJ, Trachtenberg JT (2012) Associative fear learning enhances sparse network coding in primary sensory cortex. Neuron 75:121-132.

Geramita M, Urban, NN (2016). Postnatal Odor Exposure Increases the Strength of Interglomerular Lateral Inhibition onto Olfactory Bulb Tufted Cells. J Neurosci 36(49), 12321-12327. doi: 10.1523/JNEUROSCI.1991-16.2016.

Ghatpande AS, Gelperin A (2009) Presynaptic muscarinic receptors enhance glutamate release at the mitral/tufted to granule cell dendrodendritic synapse in the rat main olfactory bulb. J Neurophysiol 101:2052-2061.

Ghosh A, Purchase NC, Chen X, Yuan Q (2015) Norepinephrine Modulates Pyramidal Cell Synaptic Properties in the Anterior Piriform Cortex of Mice: Age-Dependent Effects of beta-adrenoceptors Front Cell Neurosci 9:450 doi:10.3389/fncel.2015.00450

Ghosh S, Larson SD, Hefzi H, Marnoy Z, Cutforth T, Dokka K, Baldwin KK (2011) Sensory maps in the olfactory cortex defined by long-range viral tracing of single neurons Nature 472:217-220 doi:10.1038/nature09945

Ghosh S, Chattarji S (2015). Neuronal encoding of the switch from specific to generalized fear. Nat Neurosci 18(1), 112-120. doi: 10.1038/nn.3888.

Gilmartin MR, McEchron, MD (2005). Single neurons in the medial prefrontal cortex of the rat exhibit tonic and phasic coding during trace fear conditioning. Behav Neurosci 119(6), 1496-1510. doi: 10.1037/0735-7044.119.6.1496.

Gomeza J, Zhang L, Kostenis E, Felder C, Bymaster F, Brodkin J, Shannon H, Xia B, Deng C, Wess J (1999) Enhancement of D1 dopamine receptor-mediated locomotor stimulation in $\mathrm{M}(4)$ muscarinic acetylcholine receptor knockout mice. Proc Natl Acad Sci U S A 96:10483-10488.

Goni R, Garcia P, Foissac S (2009) The qPCR data statistical analysis. Integromics White Paper.

Gonzalez-Lima F, Agudo J (1990). Functional reorganization of neural auditory maps by differential learning. Neuroreport 1(2), 161-164.

Gray CM, Freeman WJ, Skinner JE (1986) Chemical dependencies of learning in the rabbit olfactory bulb: acquisition of the transient spatial pattern change depends on norepinephrine Behav Neurosci 100:585-596

Graziadei PP, Graziadei GA (1979) Neurogenesis and neuron regeneration in the olfactory system of mammals. I. Morphological aspects of differentiation and structural organization of the olfactory sensory neurons J Neurocytol 8:1-18 
Griff ER, Mafhouz M, Chaput MA (2008a). Comparison of identified mitral and tufted cells in freely breathing rats: II. Odor-evoked responses. Chem Senses 33(9), 793802. doi: 10.1093/chemse/bjn040.

Griff ER, Mafhouz M, Perrut A, Chaput MA (2008b). Comparison of identified mitral and tufted cells in freely breathing rats: I. Conduction velocity and spontaneous activity. Chem Senses 33(9), 779-792. doi: 10.1093/chemse/bjn041.

Grossman SE, Fontanini A., Wieskopf JS, Katz DB (2008). Learning-related plasticity of temporal coding in simultaneously recorded amygdala-cortical ensembles. J Neurosci 28(11), 2864-2873. doi: 10.1523/JNEUROSCI.4063-07.2008.

Grosso A, Cambiaghi M, Concina G, Sacco T, Sacchetti B (2015) Auditory cortex involvement in emotional learning and memory Neuroscience 299:45-55 doi:10.1016/j.neuroscience.2015.04.068

Gutierrez-Mecinas M, Crespo C, Blasco-Ibanez JM, Gracia-Llanes FJ, Marques-Mari AI, Nacher J, Varea E, Martinez-Guijarro FJ (2005) Distribution of D2 dopamine receptor in the olfactory glomeruli of the rat olfactory bulb. Eur J Neurosci 22:1357-1367.

Haberly LB, Price JL (1978) Association and commissural fiber systems of the olfactory cortex of the rat. II. Systems originating in the olfactory peduncle. J Comp Neurol 181:781-807.

Halasz N, Ljungdahl A, Hokfelt T, Johansson O, Goldstein M, Park D, Biberfeld P (1977) Transmitter histochemistry of the rat olfactory bulb. I. Immunohistochemical localization of monoamine synthesizing enzymes. Support for intrabulbar, periglomerular dopamine neurons. Brain Res 126:455-474.

Hamada S, Senzaki K, Hamaguchi-Hamada K, Tabuchi K, Yamamoto H, Yamamoto T, Yoshikawa S, Okano H, Okado N (1998) Localization of 5-HT2A receptor in rat cerebral cortex and olfactory system revealed by immunohistochemistry using two antibodies raised in rabbit and chicken Brain Res Mol Brain Res 54:199-211

Hardy A, Palouzier-Paulignan B, Duchamp A, Royet JP, Duchamp-Viret P (2005) 5Hydroxytryptamine action in the rat olfactory bulb: in vitro electrophysiological patch-clamp recordings of juxtaglomerular and mitral cells. Neuroscience 131:717-731.

Harley CW, Darby-King A, McCann J, McLean JH (2006) Beta1-adrenoceptor or alpha1-adrenoceptor activation initiates early odor preference learning in rat pups: support for the mitral cell/cAMP model of odor preference learning Learn Mem 13:8-13 doi:10.1101/lm.62006

Hasselmo ME, Anderson BP, Bower JM (1992) Cholinergic modulation of cortical associative memory function J Neurophysiol 67:1230-1246 doi:10.1152/jn.1992.67.5.1230

Hasselmo ME, Barkai E (1995) Cholinergic modulation of activity-dependent synaptic plasticity in the piriform cortex and associative memory function in a network biophysical simulation J Neurosci 15:6592-6604

Hegoburu C, Sevelinges Y, Thevenet M, Gervais R, Parrot S, Mouly AM (2009) Differential dynamics of amino acid release in the amygdala and olfactory cortex during odor fear acquisition as revealed with simultaneous high temporal resolution microdialysis Learn Mem 16:687-697 doi:10.1101/1m.1584209 
Heinbockel T, Hamilton KA, Ennis M (2007) Group I metabotropic glutamate receptors are differentially expressed by two populations of olfactory bulb granule cells. $\mathrm{J}$ Neurophysiol 97:3136-3141.

Heinbockel T, Heyward P, Conquet F, Ennis M (2004) Regulation of main olfactory bulb mitral cell excitability by metabotropic glutamate receptor mGluR1. J Neurophysiol 92:3085-3096.

Heiney SA, Wohl MP, Chettih SN, Ruffolo LI, Medina JF (2014) Cerebellar-dependent expression of motor learning during eyeblink conditioning in head-fixed mice. $\mathrm{J}$ Neurosci 34:14845-14853.

Hellier JL, Arevalo NL, Smith L, Xiong KN, Restrepo D (2012) alpha7-Nicotinic acetylcholine receptor: role in early odor learning preference in mice PLoS One 7:e35251 doi:10.1371/journal.pone.0035251

Herlenius E, Lagercrantz H (2004) Development of neurotransmitter systems during critical periods Exp Neurol 190 Suppl 1:S8-21

doi:10.1016/j.expneurol.2004.03.027

Herry C, Ciocchi S, Senn V, Demmou L, Muller C, Luthi A (2008) Switching on and off fear by distinct neuronal circuits. Nature 454:600-606.

Holahan MR, Rekart JL, Sandoval J, Routtenberg A (2006). Spatial learning induces presynaptic structural remodeling in the hippocampal mossy fiber system of two rat strains. Hippocampus 16(6), 560-570. doi: 10.1002/hipo.20185.

Homanics GE, DeLorey TM, Firestone LL, Quinlan JJ, Handforth A, Harrison NL, Krasowski MD, Rick CE, Korpi ER, Makela R, Brilliant MH, Hagiwara N, Ferguson C, Snyder K, Olsen RW (1997) Mice devoid of gamma-aminobutyrate type A receptor beta3 subunit have epilepsy, cleft palate, and hypersensitive behavior. Proc Natl Acad Sci U S A 94:4143-4148.

Hu H, Real E, Takamiya K, Kang MG, Ledoux J, Huganir RL, Malinow R (2007) Emotion enhances learning via norepinephrine regulation of AMPA-receptor trafficking Cell 131:160-173 doi:10.1016/j.cell.2007.09.017

Huang L, Ung K, Garcia I, Quast KB, Cordiner K, Saggau P, Arenkiel BR (2016) Task Learning Promotes Plasticity of Interneuron Connectivity Maps in the Olfactory Bulb. J Neurosci 36:8856-8871.

Igarashi KM, Ieki N, An M, Yamaguchi Y, Nagayama S, Kobayakawa K, Kobayakawa R, Tanifui M, Sakano H, Chen WR, Mori K (2012) Parallel mitral and tufted cell pathways route distinct odor information to different targets in the olfactory cortex J Neurosci 32:7970-7985 doi:10.1523/JNEUROSCI.0154-12.2012

Iliadi KG, Iliadi N, Boulianne GL (2017) Drosophila mutants lacking octopamine exhibit impairment in aversive olfactory associative learning Eur J Neurosci 46:20802087 doi: $10.1111 /$ ejn. 13654

Illig KR, Wilson DA (2009) Olfactory cortex: Comparative anatomy. In: Squire L (ed) The Encyclopedia of Neuroscience. 1st edn. Springer,

Izquierdo I, Furini CR, Myskiw JC (2016) Fear Memory Physiol Rev 96:695-750 doi:10.1152/physrev.00018.2015

Jasnow AM, Lynch JF, 3rd, Gilman TL, Riccio DC (2017). Perspectives on fear generalization and its implications for emotional disorders. J Neurosci Res 95(3), 821-835. doi: 10.1002/jnr.23837. 
Jenkins WM, Merzenich MM, Ochs MT, Allard T, Guic-Robles E (1990). Functional reorganization of primary somatosensory cortex in adult owl monkeys after behaviorally controlled tactile stimulation. J Neurophysiol 63(1), 82-104. doi: 10.1152/jn.1990.63.1.82.

Jerome D, Hou Q, Yuan Q (2012) Interaction of NMDA receptors and L-type calcium channels during early odor preference learning in rats. Eur J Neurosci 36:31343141.

Jerusalinsky D, Kornisiuk E, Izquierdo I (1997) Cholinergic neurotransmission and synaptic plasticity concerning memory processing Neurochem Res 22:507-515

Jian K, Cifelli P, Pignatelli A, Frigato E, Belluzzi O (2010) Metabotropic glutamate receptors 1 and 5 differentially regulate bulbar dopaminergic cell function. Brain Res 1354:47-63.

Johansen JP, Cain CK, Ostroff LE, LeDoux JE (2011) Molecular mechanisms of fear learning and memory. Cell 147:509-524.

Johnson BA, Woo CC, Duong H, Nguyen V, Leon M (1995) A learned odor evokes an enhanced Fos-like glomerular response in the olfactory bulb of young rats. Brain Res 699:192-200.

Johnson O, Becnel J, Nichols CD (2011) Serotonin receptor activity is necessary for olfactory learning and memory in Drosophila melanogaster Neuroscience 192:372-381 doi:10.1016/j.neuroscience.2011.06.058

Jones SV, Choi DC, Davis M, Ressler KJ (2008) Learning-dependent structural plasticity in the adult olfactory pathway J Neurosci 28:13106-13111 doi:10.1523/JNEUROSCI.4465-08.2008

Jones SV, Stanek-Rattiner L, Davis M, Ressler KJ (2007) Differential regional expression of brain-derived neurotrophic factor following olfactory fear learning Learn Mem 14:816-820 doi:10.1101/lm.781507

Kaas JH, Krubitzer LA, Chino YM, Langston AL, Polley EH, Blair N (1990). Reorganization of retinotopic cortical maps in adult mammals after lesions of the retina. Science 248(4952), 229-231.

Kadohisa M, Wilson DA (2006) Separate encoding of identity and similarity of complex familiar odors in piriform cortex. Proc Natl Acad Sci U S A 103:15206-15211.

Kamimura K, Ueno K, Nakagawa J, Hamada R, Saitoe M, Maeda N (2013) Perlecan regulates bidirectional Wnt signaling at the Drosophila neuromuscular junction. J Cell Biol 200:219-233.

Kaneko N, Okano H, Sawamoto K (2006) Role of the cholinergic system in regulating survival of newborn neurons in the adult mouse dentate gyrus and olfactory bulb. Genes Cells 11:1145-1159.

Kapoor V, Provost AC, Agarwal P, Murthy VN (2016) Activation of raphe nuclei triggers rapid and distinct effects on parallel olfactory bulb output channels. Nat Neurosci 19:271-282.

Kasa P, Hlavati I, Dobo E, Wolff A, Joo F, Wolff JR (1995) Synaptic and non-synaptic cholinergic innervation of the various types of neurons in the main olfactory bulb of adult rat: immunocytochemistry of choline acetyltransferase Neuroscience 67:667-677 
Kass MD, McGann JP (2017) Persistent, generalized hypersensitivity of olfactory bulb interneurons after olfactory fear generalization Neurobiol Learn Mem 146:47-57 doi:10.1016/j.nlm.2017.11.004

Kass MD, Rosenthal MC, Pottackal J, McGann JP (2013) Fear learning enhances neural responses to threat-predictive sensory stimuli Science 342:1389-1392 doi:10.1126/science. 1244916

Kato HK, Chu MW, Isaacson JS, Komiyama T (2012) Dynamic sensory representations in the olfactory bulb: modulation by wakefulness and experience. Neuron 76:962975.

Kaupmann K, Bruns C, Hoyer D, Seuwen K, Lubbert H (1993) Distribution and second messenger coupling of four somatostatin receptor subtypes expressed in brain. FEBS Lett 331:53-59.

Kay LM (2014) Circuit oscillations in odor perception and memory Prog Brain Res 208:223-251 doi:10.1016/B978-0-444-63350-7.00009-7

Kay LM, Beshel J (2010) A beta oscillation network in the rat olfactory system during a 2-alternative choice odor discrimination task. J Neurophysiol 104:829-839.

Kelahan AM, Doetsch GS (1984). Time-dependent changes in the functional organization of somatosensory cerebral cortex following digit amputation in adult raccoons. Somatosens Res 2(1), 49-81.

Kerr MA, Belluscio L (2006) Olfactory experience accelerates glomerular refinement in the mammalian olfactory bulb Nat Neurosci 9:484-486 doi:10.1038/nn1673

Kikuta S, Fletcher ML, Homma R, Yamasoba T, Nagayama S (2013). Odorant response properties of individual neurons in an olfactory glomerular module. Neuron 77(6), 1122-1135. doi: 10.1016/j.neuron.2013.01.022.

Kolb B, Gibb R (2014) Searching for the principles of brain plasticity and behavior Cortex 58:251-260 doi:10.1016/j.cortex.2013.11.012

Koyama Y, Kondo M, Shimada S (2017) Building a 5-HT3A Receptor Expression Map in the Mouse Brain. Sci Rep 7:42884.

Kroon JA, Carobrez AP (2009) Olfactory fear conditioning paradigm in rats: effects of midazolam, propranolol or scopolamine Neurobiol Learn Mem 91:32-40 doi:10.1016/j.nlm.2008.10.007

Kudo K, Qiao CX, Kanba S, Arita J (2004) A selective increase in phosphorylation of cyclic AMP response element-binding protein in hippocampal CA1 region of male, but not female, rats following contextual fear and passive avoidance conditioning Brain Res 1024:233-243 doi:10.1016/j.brainres.2004.08.007

Landers MS, Sullivan RM (2012) The development and neurobiology of infant attachment and fear Dev Neurosci 34:101-114 doi:10.1159/000336732

Lashley KS, Wade M (1946). The Pavlovian theory of generalization. Psychol Rev 53, $72-87$.

Laurie DJ, Seeburg PH, Wisden W (1992) The distribution of 13 GABAA receptor subunit mRNAs in the rat brain. II. Olfactory bulb and cerebellum. J Neurosci 12:1063-1076.

Le Jeune H, Aubert I, Jourdan F, Quirion R (1995) Comparative laminar distribution of various autoradiographic cholinergic markers in adult rat main olfactory bulb. J Chem Neuroanat 9:99-112. 
Le Jeune H, Jourdan F (1991) Postnatal development of cholinergic markers in the rat olfactory bulb: a histochemical and immunocytochemical study J Comp Neurol 314:383-395 doi:10.1002/cne.903140212

Lebel D, Grossman Y, Barkai E (2001) Olfactory learning modifies predisposition for long-term potentiation and long-term depression induction in the rat piriform (olfactory) cortex Cereb Cortex 11:485-489

Lepousez G, Csaba Z, Bernard V, Loudes C, Videau C, Lacombe J, Epelbaum J, Viollet C (2010) Somatostatin interneurons delineate the inner part of the external plexiform layer in the mouse main olfactory bulb. J Comp Neurol 518:1976-1994.

Lerea LS, McNamara JO (1993) Ionotropic glutamate receptor subtypes activate c-fos transcription by distinct calcium-requiring intracellular signaling pathways. Neuron 10:31-41.

Lethbridge R, Hou Q, Harley CW, Yuan Q (2012) Olfactory bulb glomerular NMDA receptors mediate olfactory nerve potentiation and odor preference learning in the neonate rat. PLoS One 7:e35024.

Letzkus JJ, Wolff SB, Meyer EM, Tovote P, Courtin J, Herry C, Luthi A (2011) A disinhibitory microcircuit for associative fear learning in the auditory cortex. Nature 480:331-335.

Levey AI, Hersch SM, Rye DB, Sunahara RK, Niznik HB, Kitt CA, Price DL, Maggio R, Brann MR, Ciliax BJ (1993) Localization of D1 and D2 dopamine receptors in brain with subtype-specific antibodies. Proc Natl Acad Sci U S A 90:8861-8865.

Levy F, Meurisse M, Ferreira G, Thibault J, Tillet Y (1999) Afferents to the rostral olfactory bulb in sheep with special emphasis on the cholinergic, noradrenergic and serotonergic connections J Chem Neuroanat 16:245-263

Li A, Gire DH, Restrepo D (2015). Upsilon spike-field coherence in a population of olfactory bulb neurons differentiates between odors irrespective of associated outcome. J Neurosci 35(14), 5808-5822. doi: 10.1523/JNEUROSCI.400314.2015.

Li G, Cleland TA (2013) A two-layer biophysical model of cholinergic neuromodulation in olfactory bulb. J Neurosci 33:3037-3058.

Li W, Howard JD, Parrish TB, Gottfried JA (2008) Aversive learning enhances perceptual and cortical discrimination of indiscriminable odor cues. Science 319:1842-1845.

Liguz-Lecznar M, Urban-Ciecko J, Kossut M (2016) Somatostatin and SomatostatinContaining Neurons in Shaping Neuronal Activity and Plasticity. Front Neural Circuits 10:48.

Lima J, Sharp T, Bannerman DM, McHugh SB (2019). Enhanced discriminative aversive learning and amygdala responsivity in 5-HT transporter mutant mice. Transl Psychiatry 9(1), 139. doi: 10.1038/s41398-019-0476-8.

Linster C, Cleland TA (2016) Neuromodulation of olfactory transformations. Curr Opin Neurobiol 40:170-177.

Linster C, Hasselmo ME (2001) Neuromodulation and the functional dynamics of piriform cortex Chem Senses 26:585-594

Lissek S, Kaczkurkin AN, Rabin S, Geraci M, Pine DS, Grillon C (2014) Generalized anxiety disorder is associated with overgeneralization of classically conditioned fear. Biol Psychiatry 75:909-915. 
Lissek S, Rabin S, Heller RE, Lukenbaugh D, Geraci M, Pine DS, Grillon C (2010) Overgeneralization of conditioned fear as a pathogenic marker of panic disorder. Am J Psychiatry 167:47-55.

Litaudon P, Mouly AM, Sullivan R, Gervais R, Cattarelli M (1997) Learning-induced changes in rat piriform cortex activity mapped using multisite recording with voltage sensitive dye Eur J Neurosci 9:1593-1602

Liu S, Shao Z, Puche A, Wachowiak M, Rothermel M, Shipley MT (2015) Muscarinic receptors modulate dendrodendritic inhibitory synapses to sculpt glomerular output. J Neurosci 35:5680-5692.

Livak KJ, Schmittgen TD (2001) Analysis of relative gene expression data using realtime quantitative PCR and the 2(-Delta Delta C(T)) Method. Methods 25:402408.

Lottem E, Lorincz ML, Mainen ZF (2016) Optogenetic Activation of Dorsal Raphe Serotonin Neurons Rapidly Inhibits Spontaneous But Not Odor-Evoked Activity in Olfactory Cortex J Neurosci 36:7-18 doi:10.1523/JNEUROSCI.3008-15.2016

Lucaites VL, Krushinski JH, Schaus JM, Audia JE, Nelson DL (2005) [3H]LY334370, a novel radioligand for the 5-HT1F receptor. II. Autoradiographic localization in rat, guinea pig, monkey and human brain. Naunyn Schmiedebergs Arch Pharmacol 371:178-184.

Lugo JN, Smith GD, Holley AJ (2014) Trace fear conditioning in mice J Vis Exp doi: $10.3791 / 51180$

Luscher C, Malenka RC (2012) NMDA receptor-dependent long-term potentiation and long-term depression (LTP/LTD) Cold Spring Harb Perspect Biol 4 doi:10.1101/cshperspect.a005710

Ma M, Luo M (2012) Optogenetic activation of basal forebrain cholinergic neurons modulates neuronal excitability and sensory responses in the main olfactory bulb. J Neurosci 32:10105-10116.

Mackay-Sim A, Kittel PW (1991) On the Life Span of Olfactory Receptor Neurons Eur J Neurosci 3:209-215

Macrides F, Davis BJ, Youngs WM, Nadi NS, Margolis FL (1981) Cholinergic and catecholaminergic afferents to the olfactory bulb in the hamster: a neuroanatomical, biochemical, and histochemical investigation. J Comp Neurol 203:495-514.

Mair RG, Gellman RL, Gesteland RC (1982) Postnatal proliferation and maturation of olfactory bulb neurons in the rat Neuroscience 7:3105-3116

Maity S, Rah S, Sonenberg N, Gkogkas CG, Nguyen PV (2015) Norepinephrine triggers metaplasticity of LTP by increasing translation of specific mRNAs Learn Mem 22:499-508 doi: $10.1101 / \mathrm{lm} .039222 .115$

Majak K, Ronkko S, Kemppainen S, Pitkanen A (2004) Projections from the amygdaloid complex to the piriform cortex: A PHA-L study in the rat J Comp Neurol 476:414-428 doi:10.1002/cne.20233

Mandairon N, Ferretti CJ, Stack CM, Rubin DB, Cleland TA, Linster C (2006) Cholinergic modulation in the olfactory bulb influences spontaneous olfactory discrimination in adult rats. Eur J Neurosci 24:3234-3244. 
Marchetti E, Dumuis A, Bockaert J, Soumireu-Mourat B, Roman FS (2000) Differential modulation of the 5-HT(4) receptor agonists and antagonist on rat learning and memory Neuropharmacology 39:2017-2027

Marcucci F, Maier-Balough E, Zou DJ, Firestein S (2011) Exuberant growth and synapse formation of olfactory sensory neuron axonal arborizations J Comp Neurol 519:3713-3726 doi:10.1002/cne.22684

Maren S (2001) Neurobiology of Pavlovian fear conditioning Annu Rev Neurosci 24:897-931 doi:10.1146/annurev.neuro.24.1.897

Maren S (2003a) What the amygdala does and doesn't do in aversive learning. Learn Mem 10:306-308.

Maren S (2003b) The amygdala, synaptic plasticity, and fear memory. Ann N Y Acad Sci 985:106-113.

Maren S (2005) Synaptic mechanisms of associative memory in the amygdala. Neuron 47:783-786.

Martin-Cora FJ, Pazos A (2004) Autoradiographic distribution of 5-HT7 receptors in the human brain using $[3 \mathrm{H}]$ mesulergine: comparison to other mammalian species. $\mathrm{Br}$ J Pharmacol 141:92-104.

McAfee SS, Ogg MC, Ross JM, Liu Y, Fletcher ML, Heck DH (2016) Minimally invasive highly precise monitoring of respiratory rhythm in the mouse using an epithelial temperature probe. J Neurosci Methods 263:89-94.

McLean JH, Darby-King A, Hodge E (1996) 5-HT2 receptor involvement in conditioned olfactory learning in the neonate rat pup Behav Neurosci 110:1426-1434

McLean JH, Darby-King A, Paterno GD (1995) Localization of 5-HT2A receptor mRNA by in situ hybridization in the olfactory bulb of the postnatal rat J Comp Neurol 353:371-378 doi:10.1002/cne.903530305

McLean JH, Darby-King A, Sullivan RM, King SR (1993) Serotonergic influence on olfactory learning in the neonate rat Behav Neural Biol 60:152-162

McLean JH, Harley CW, Darby-King A, Yuan Q (1999) pCREB in the neonate rat olfactory bulb is selectively and transiently increased by odor preferenceconditioned training Learn Mem 6:608-618

McLean JH, Shipley MT (1987) Serotonergic afferents to the rat olfactory bulb: I. Origins and laminar specificity of serotonergic inputs in the adult rat J Neurosci 7:3016-3028

McLean JH, Shipley MT (1991) Postnatal development of the noradrenergic projection from locus coeruleus to the olfactory bulb in the rat J Comp Neurol 304:467-477 doi: $10.1002 /$ cne. 903040310

McNamara AM, Magidson PD, Linster C, Wilson DA, Cleland TA (2008) Distinct neural mechanisms mediate olfactory memory formation at different timescales. Learn Mem 15:117-125.

Mechawar N, Saghatelyan A, Grailhe R, Scoriels L, Gheusi G, Gabellec MM, Lledo PM, Changeux JP (2004) Nicotinic receptors regulate the survival of newborn neurons in the adult olfactory bulb. Proc Natl Acad Sci U S A 101:9822-9826.

Meneses A (1998) Physiological, pathophysiological and therapeutic roles of 5-HT systems in learning and memory. Rev Neurosci 9:275-289. 
Meneses A, Terron JA, Hong E (1998) Involvement of 5-HT1A receptors in the consolidation of learning in cognitively impaired rats. Ann N Y Acad Sci 861:286-287.

Menzel R (2001) Searching for the memory trace in a mini-brain, the honeybee Learn Mem 8:53-62 doi:10.1101/lm.38801

Merzenich MM, Kaas JH, Wall JT, Sur M, Nelson RJ, Felleman DJ (1983). Progression of change following median nerve section in the cortical representation of the hand in areas $3 \mathrm{~b}$ and 1 in adult owl and squirrel monkeys. Neuroscience 10(3), 639-665.

Miyamichi K Amat F, Moussavi F, Wang C, Wichersham I, Wall NR, Taniguchi H, Tasic B, Huang ZJ, He Z, Callaway EM, Horowitz MA, Luo L (2011) Cortical representations of olfactory input by trans-synaptic tracing Nature 472:191-196 doi:10.1038/nature09714

Mlinar B, Stocca G, Corradetti R (2015) Endogenous serotonin facilitates hippocampal long-term potentiation at CA3/CA1 synapses J Neural Transm (Vienna) 122:177185 doi:10.1007/s00702-014-1246-7

Modarresi S, Mukherjee B, McLean JH, Harley CW, Yuan Q (2016) CaMKII mediates stimulus specificity in early odor preference learning in rats $\mathrm{J}$ Neurophysiol 116:404-410 doi:10.1152/jn.00176.2016

Mogilner A, Grossman JA, Ribary U, Joliot M, Volkmann J, Rapaport D, Beasley RW, Llinas RR (1993). Somatosensory cortical plasticity in adult humans revealed by magnetoencephalography. Proc Natl Acad Sci U S A 90(8), 3593-3597.

Montague AA, Greer CA (1999) Differential distribution of ionotropic glutamate receptor subunits in the rat olfactory bulb. J Comp Neurol 405:233-246.

Mori K, Takahashi YK, Igarashi KM, Yamaguchi M (2006) Maps of odorant molecular features in the Mammalian olfactory bulb Physiol Rev 86:409-433 doi:10.1152/physrev.00021.2005

Moriceau S, Sullivan RM (2004) Corticosterone influences on Mammalian neonatal sensitive-period learning Behav Neurosci 118:274-281 doi:10.1037/07357044.118.2.274

Morrison DJ, Rashid AJ, Yiu AP, Yan C, Frankland PW, Josselyn SA (2016) Parvalbumin interneurons constrain the size of the lateral amygdala engram Neurobiol Learn Mem 135:91-99 doi:10.1016/j.nlm.2016.07.007

Morrison FG, Dias BG, Ressler KJ (2015) Extinction reverses olfactory fear-conditioned increases in neuron number and glomerular size Proc Natl Acad Sci U S A 112:12846-12851 doi:10.1073/pnas. 1505068112

Morrison GL, Fontaine CJ, Harley CW, Yuan Q (2013) A role for the anterior piriform cortex in early odor preference learning: evidence for multiple olfactory learning structures in the rat pup J Neurophysiol 110:141-152 doi:10.1152/jn.00072.2013

Mott DD, Rojas A, Fisher JL, Dingledine RJ, Benveniste M (2010) Subunit-specific desensitization of heteromeric kainate receptors. J Physiol 588:683-700.

Mouly AM, Fort A, Ben-Boutayab N, Gervais R (2001) Olfactory learning induces differential long-lasting changes in rat central olfactory pathways Neuroscience 102:11-21

Mouret A, Murray K, Lledo PM (2009) Centrifugal drive onto local inhibitory interneurons of the olfactory bulb. Ann N Y Acad Sci 1170:239-254. 
Mukai I, Kim D, Fukunaga M, Japee S, Marrett S, Ungerleider LG (2007) Activations in visual and attention-related areas predict and correlate with the degree of perceptual learning. J Neurosci 27:11401-11411.

Mundy ME, Downing PE, Honey RC, Singh KD, Graham KS, Dwyer DM (2014) Brain correlates of experience-dependent changes in stimulus discrimination based on the amount and schedule of exposure. PLoS One 9:e101011.

Mwilaria EK, Ghatak C, Daly KC (2008) Disruption of GABAA in the insect antennal lobe generally increases odor detection and discrimination thresholds. Chem Senses 33:267-281.

Nagayama S, Enerva A, Fletcher ML, Masurkar AV, Igarashi KM, Mori K, Chen WR (2010) Differential axonal projection of mitral and tufted cells in the mouse main olfactory system Front Neural Circuits 4 doi:10.3389/fncir.2010.00120

Nagayama S, Takahashi YK., Yoshihara Y, Mori K (2004). Mitral and tufted cells differ in the decoding manner of odor maps in the rat olfactory bulb. J Neurophysiol 91(6), 2532-2540. doi: 10.1152/jn.01266.2003.

Nakanishi S, Masu M, Bessho Y, Nakajima Y, Hayashi Y, Shigemoto R (1994) Molecular diversity of glutamate receptors and their physiological functions. EXS 71:71-80.

Nasir SM, Darainy M, Ostry DJ (2013). Sensorimotor adaptation changes the neural coding of somatosensory stimuli. J Neurophysiol 109(8), 2077-2085. doi: 10.1152/jn.00719.2012.

Neant-Fery M, Peres E, Nasrallah C, Kessner M, Gribaudo S, Greer C, Didier A, Trembleau A, Caille I (2012) A role for dendritic translation of CaMKIIalpha mRNA in olfactory plasticity. PLoS One 7:e40133.

Nickell WT, Shipley MT (1988) Two anatomically specific classes of candidate cholinoceptive neurons in the rat olfactory bulb. J Neurosci 8:4482-4491.

Nocera S, Simon A, Fiquet O, Chen Y, Gascuel J, Datiche FA, Schneider N, Epelbaum J, Viollet C (2019) Somatostatin Serves a Modulatory Role in the Mouse Olfactory Bulb: Neuroanatomical and Behavioral Evidence. Frontiers in Behavioral Neuroscience.

Nunes D, Kuner T (2015) Disinhibition of olfactory bulb granule cells accelerates odour discrimination in mice. Nat Commun 6:8950.

Nunez-Parra A., Li A, Restrepo D (2014). Coding odor identity and odor value in awake rodents. Prog Brain Res 208, 205-222. doi: 10.1016/B978-0-444-63350-7.000085.

Nusser Z, Kay LM, Laurent G, Homanics GE, Mody I (2001) Disruption of GABA(A) receptors on GABAergic interneurons leads to increased oscillatory power in the olfactory bulb network. J Neurophysiol 86:2823-2833.

O'Neill M, Schultz W. (2018). Predictive coding of the statistical parameters of uncertain rewards by orbitofrontal neurons. Behav Brain Res 355, 90-94. doi: 10.1016/j.bbr.2018.04.041.

Ogg MC, Bendahamane M, Fletcher ML (2015) Habituation of glomerular responses in the olfactory bulb following prolonged odor stimulation reflects reduced peripheral input. Front Mol Neurosci 8:53. 
Ogg MC, Ross JM, Bendahmane M, Fletcher ML (2018) Olfactory bulb acetylcholine release dishabituates odor responses and reinstates odor investigation. Nature Communications 9:1868.

Ojima H, Mori K, Kishi K (1984) The trajectory of mitral cell axons in the rabbit olfactory cortex revealed by intracellular HRP injection J Comp Neurol 230:77-87 doi:10.1002/cne.902300107

Okutani F, Yagi F, Kaba H (1999) Gabaergic control of olfactory learning in young rats. Neuroscience 93:1297-1300.

Okutani F, Zhang JJ, Yagi F, Kaba H (2002) Non-specific olfactory aversion induced by intrabulbar infusion of the GABA(A) receptor antagonist bicuculline in young rats. Neuroscience 112:901-906.

Okutani F, Zhang JJ, Otsuka T, Yagi F, Kaba H (2003) Modulation of olfactory learning in young rats through intrabulbar GABA(B) receptors Eur J Neurosci 18:20312036

Onali P, Aasen AJ, Olianas MC (1994) Antagonism by (R)- and (S)-trihexyphenidyl of muscarinic stimulation of adenylyl cyclase in rat olfactory bulb and inhibition in striatum and heart. Br J Pharmacol 113:775-780.

Osinski BL, Kim A, Xiao W, Mehta NM, Kay LM (2018) Pharmacological manipulation of the olfactory bulb modulates beta oscillations: testing model predictions. J Neurophysiol 120:1090-1106.

Otazu GH, Chae H, Davis MB, Albeanu DF (2015) Cortical Feedback Decorrelates Olfactory Bulb Output in Awake Mice. Neuron 86:1461-1477.

Otto T, Cousens G, Rajewski K (1997) Odor-guided fear conditioning in rats: 1. Acquisition, retention, and latent inhibition. Behav Neurosci 111:1257-1264.

Pager J, Royet JP (1976) Some effects of conditioned aversion on food intake and olfactory bulb electrical responses in the rat J Comp Physiol Psychol 90:67-77

Pandipati S, Schoppa NE (2012) Age-dependent adrenergic actions in the main olfactory bulb that could underlie an olfactory-sensitive period J Neurophysiol 108:19992007 doi:10.1152/jn.00322.2012

Panzanelli P, Fritschy JM, Yanagawa Y, Obata K, Sassoe-Pognetto M (2007) GABAergic phenotype of periglomerular cells in the rodent olfactory bulb. $\mathrm{J}$ Comp Neurol 502:990-1002.

Park M, Salgado JM, Ostroff L, Helton TD, Robinson CG, Harris KM, Ehlers MD (2006) Plasticity-induced growth of dendritic spines by exocytic trafficking from recycling endosomes Neuron 52:817-830 doi:10.1016/j.neuron.2006.09.040

Parsa PV, D'Souza RD, Vijayaraghavan S (2015) Signaling between periglomerular cells reveals a bimodal role for GABA in modulating glomerular microcircuitry in the olfactory bulb. Proc Natl Acad Sci U S A 112:9478-9483.

Pascual-Leone A, Torres F (1993). Plasticity of the sensorimotor cortex representation of the reading finger in Braille readers. Brain 116 ( Pt 1), 39-52.

Patil MM, Linster C, Lubenov E, Hasselmo ME (1998) Cholinergic agonist carbachol enables associative long-term potentiation in piriform cortex slices $\mathrm{J}$ Neurophysiol 80:2467-2474 doi:10.1152/jn.1998.80.5.2467

Pavesi E, Gooch A, Lee E, Fletcher ML (2012) Cholinergic modulation during acquisition of olfactory fear conditioning alters learning and stimulus generalization in mice Learn Mem 20:6-10 doi:10.1101/1m.028324.112 
Pavlov IP (1927) Conditioned Reflexes. Oxford University Press,

Pazos A, Palacios JM (1985) Quantitative autoradiographic mapping of serotonin receptors in the rat brain. I. Serotonin-1 receptors. Brain Res 346:205-230.

Pazos A, Cortes R, Palacios JM (1985) Quantitative autoradiographic mapping of serotonin receptors in the rat brain. II. Serotonin-2 receptors. Brain Res 346:231249.

Perez J, Hoyer D (1995) Co-expression of somatostatin SSTR-3 and SSTR-4 receptor messenger RNAs in the rat brain. Neuroscience 64:241-253.

Petzold GC, Hagiwara A, Murthy VN (2009) Serotonergic modulation of odor input to the mammalian olfactory bulb. Nat Neurosci 12:784-791.

Pfaffl MW (2001) A new mathematical model for relative quantification in real-time RTPCR. Nucleic Acids Res 29:e45.

Pignatelli A, Belluzzi O (2008) Cholinergic modulation of dopaminergic neurons in the mouse olfactory bulb. Chem Senses 33:331-338.

Pnevmatikakis EA, Giovannucci A (2017). NoRMCorre: An online algorithm for piecewise rigid motion correction of calcium imaging data. J Neurosci Methods 291, 83-94. doi: 10.1016/j.jneumeth.2017.07.031.

Pompeiano M, Palacios JM, Mengod G (1994) Distribution of the serotonin 5-HT2 receptor family mRNAs: comparison between 5 -HT2A and 5-HT2C receptors. Brain Res Mol Brain Res 23:163-178.

Pressler RT, Inoue T, Strowbridge BW (2007) Muscarinic receptor activation modulates granule cell excitability and potentiates inhibition onto mitral cells in the rat olfactory bulb. J Neurosci 27:10969-10981.

Price JL, Powell TP (1970) An experimental study of the origin and course of the centrifugal fibres to the olfactory bulb in the rat. J Anat 107:215-237.

Price TL, Darby-King A, Harley CW, McLean JH (1998) Serotonin plays a permissive role in conditioned olfactory learning induced by norepinephrine in the neonate rat. Behav Neurosci 112:1430-1437.

Quian Quiroga R (2016). Neuronal codes for visual perception and memory. Neuropsychologia 83, 227-241. doi: 10.1016/j.neuropsychologia.2015.12.016.

Quinlan EM, Lebel D, Brosh I, Barkai E (2004) A molecular mechanism for stabilization of learning-induced synaptic modifications Neuron 41:185-192

Raineki C, Shionoya K, Sander K, Sullivan RM (2009) Ontogeny of odor-LiCl vs. odorshock learning: similar behaviors but divergent ages of functional amygdala emergence Learn Mem 16:114-121 doi:10.1101/lm.977909

Ramirez-Gordillo D, Ma M, Restrepo D (2018) Precision of Classification of Odorant Value by the Power of Olfactory Bulb Oscillations Is Altered by Optogenetic Silencing of Local Adrenergic Innervation Front Cell Neurosci 12:48 doi:10.3389/fncel.2018.00048

Rath L, Giovanni Galizia C, Szyszka P (2011) Multiple memory traces after associative learning in the honey bee antennal lobe. Eur J Neurosci 34:352-360.

Ravel N, Elaagouby A, Gervais R (1994) Scopolamine injection into the olfactory bulb impairs short-term olfactory memory in rats. Behav Neurosci 108:317-324.

Ravel N, Akaoka H, Gervais R, Chouvet G (1990) The effect of acetylcholine on rat olfactory bulb unit activity. Brain Res Bull 24:151-155. 
Rea MA, Nurnberger JI, Jr. (1986) Evidence for developmental synaptic regression of cholinergic afferents to the rat main olfactory bulb Brain Res 389:233-237

Resnik J, Paz R (2015) Fear generalization in the primate amygdala. Nat Neurosci 18:188-190.

Ressler KJ, Sullivan SL, Buck LB (1994) Information coding in the olfactory system: evidence for a stereotyped and highly organized epitope map in the olfactory bulb Cell 79:1245-1255

Reuveni I, Saar D, Barkai E (2013) A novel whole-cell mechanism for long-term memory enhancement PLoS One 8:e68131 doi:10.1371/journal.pone.0068131

Ribeiro AM, Barbosa FF, Munguba H, Costa MS, Cavalcante JS, Silva RH (2011) Basolateral amygdala inactivation impairs learned (but not innate) fear response in rats. Neurobiol Learn Mem 95:433-440.

Robertson D, Irvine DR (1989). Plasticity of frequency organization in auditory cortex of guinea pigs with partial unilateral deafness. J Comp Neurol 282(3), 456-471. doi: 10.1002/cne.902820311.

Rogan MT, Staubli UV, LeDoux JE (1997) Fear conditioning induces associative longterm potentiation in the amygdala. Nature 390:604-607.

Ross JM, Fletcher ML (2018a) Learning-Dependent and -Independent Enhancement of Mitral/Tufted Cell Glomerular Odor Responses Following Olfactory Fear Conditioning in Awake Mice J Neurosci 38:4623-4640 doi:10.1523/JNEUROSCI.3559-17.2018

Ross JM, Fletcher ML (2018b) Aversive learning-induced plasticity throughout the adult mammalian olfactory system: insights across development. J Bioenerg Biomembr, doi:10.1007/s10863-018-9770-z

Ross JM, Fletcher ML (2018c) Assessing Classical Olfactory Fear Conditioning by Behavioral Freezing in Mice. Bio-protocol 8:e3013.

Rosselli-Austin L, Altman J (1979) The postnatal development of the main olfactory bulb of the rat J Dev Physiol 1:295-313

Roth TL, Moriceau S, Sullivan RM (2006) Opioid modulation of Fos protein expression and olfactory circuitry plays a pivotal role in what neonates remember Learn Mem 13:590-598 doi:10.1101/1m.301206

Roth TL, Sullivan RM (2005) Memory of early maltreatment: neonatal behavioral and neural correlates of maternal maltreatment within the context of classical conditioning Biol Psychiatry 57:823-831 doi:10.1016/j.biopsych.2005.01.032

Rothermel M, Wachowiak M (2014) Functional imaging of cortical feedback projections to the olfactory bulb. Front Neural Circuits 8:73.

Rothermel M, Carey RM, Puche A, Shipley MT, Wachowiak M (2014) Cholinergic inputs from Basal forebrain add an excitatory bias to odor coding in the olfactory bulb. J Neurosci 34:4654-4664.

Ruat M, Traiffort E, Arrang JM, Tardivel-Lacombe J, Diaz J, Leurs R, Schwartz JC (1993) A novel rat serotonin (5-HT6) receptor: molecular cloning, localization and stimulation of cAMP accumulation. Biochem Biophys Res Commun 193:268-276.

Saar D, Grossman Y, Barkai E (2001) Long-lasting cholinergic modulation underlies rule learning in rats. J Neurosci 21:1385-1392. 
Saar D, Reuveni I, Barkai E (2012) Mechanisms underlying rule learning-induced enhancement of excitatory and inhibitory synaptic transmission J Neurophysiol 107:1222-1229 doi:10.1152/jn.00356.2011

Sabri MM, Arabzadeh E (2018). Information processing across behavioral states: Modes of operation and population dynamics in rodent sensory cortex. Neuroscience 368, 214-228. doi: 10.1016/j.neuroscience.2017.09.016.

Sadrian B, Wilson DA (2015) Optogenetic Stimulation of Lateral Amygdala Input to Posterior Piriform Cortex Modulates Single-Unit and Ensemble Odor Processing Front Neural Circuits 9:81 doi:10.3389/fncir.2015.00081

Sanchez-Andrade G, James BM, Kendrick KM (2005) Neural encoding of olfactory recognition memory. J Reprod Dev 51:547-558.

Sarma AA, Richard MB, Greer CA (2011) Developmental dynamics of piriform cortex Cereb Cortex 21:1231-1245 doi:10.1093/cercor/bhq199

Saugstad JA, Kinzie JM, Shinohara MM, Segerson TP, Westbrook GL (1997) Cloning and expression of rat metabotropic glutamate receptor 8 reveals a distinct pharmacological profile. Mol Pharmacol 51:119-125.

Scheich H, Zuschratter W (1995). Mapping of stimulus features and meaning in gerbil auditory cortex with 2-deoxyglucose and c-Fos antibodies. Behav Brain Res 66(12), 195-205.

Schmidt LJ, Strowbridge BW (2014) Modulation of olfactory bulb network activity by serotonin: synchronous inhibition of mitral cells mediated by spatially localized GABAergic microcircuits. Learn Mem 21:406-416.

Schoppa NE, Westbrook GL (1997) Modulation of mEPSCs in olfactory bulb mitral cells by metabotropic glutamate receptors. J Neurophysiol 78:1468-1475.

Schwob JE, Price JL (1984a) The development of axonal connections in the central olfactory system of rats J Comp Neurol 223:177-202 doi:10.1002/cne.902230204

Schwob JE, Price JL (1984b) The development of lamination of afferent fibers to the olfactory cortex in rats, with additional observations in the adult J Comp Neurol 223:203-222 doi:10.1002/cne.902230205

Scott JW, McBride RL, Schneider SP (1980) The organization of projections from the olfactory bulb to the piriform cortex and olfactory tubercle in the rat J Comp Neurol 194:519-534 doi:10.1002/cne.901940304

Sellers KK, Bennett DV, Hutt A, Williams JH, Frohlich F. (2015). Awake vs. anesthetized: layer-specific sensory processing in visual cortex and functional connectivity between cortical areas. J Neurophysiol 113(10), 3798-3815. doi: 10.1152/jn.00923.2014.

Sevelinges Y, Gervais R, Messaoudi B, Granjon L, Mouly AM (2004) Olfactory fear conditioning induces field potential potentiation in rat olfactory cortex and amygdala Learn Mem 11:761-769 doi:10.1101/1m.83604

Sevelinges Y, Sullivan RM, Messaoudi B, Mouly AM (2008) Neonatal odor-shock conditioning alters the neural network involved in odor fear learning at adulthood. Learn Mem 15:649-656.

Shakhawat AM, Gheidi A, MacIntyre IT, Walsh ML, Harley CW, Yuan Q (2015) ArcExpressing Neuronal Ensembles Supporting Pattern Separation Require Adrenergic Activity in Anterior Piriform Cortex: An Exploration of Neural 
Constraints on Learning J Neurosci 35:14070-14075

doi:10.1523/JNEUROSCI.2690-15.2015

Shakhawat AM, Harley CW, Yuan Q (2012) Olfactory bulb alpha2-adrenoceptor activation promotes rat pup odor-preference learning via a cAMP-independent mechanism Learn Mem 19:499-502 doi:10.1101/lm.027359.112

Shen Y, Monsma FJ, Jr., Metcalf MA, Jose PA, Hamblin MW, Sibley DR (1993) Molecular cloning and expression of a 5-hydroxytryptamine7 serotonin receptor subtype. J Biol Chem 268:18200-18204.

Shepard RN (1987). Toward a universal law of generalization for psychological science. Science 237(4820), 1317-1323.

Shionoya K, Moriceau S, Lunday L, Miner C, Roth TL, Sullivan RM (2006) Development switch in neural circuitry underlying odor-malaise learning Learn Mem 13:801-808 doi:10.1101/lm.316006

Shipley MT, Ennis M (1996) Functional organization of olfactory system J Neurobiol 30:123-176 doi:10.1002/(SICI)1097-4695(199605)30:1\&1t;123::AIDNEU11\&gt;3.0.CO;2-N

Shusterman R, Smear MC, Koulakov AA, Rinberg D (2011) Precise olfactory responses tile the sniff cycle. Nat Neurosci 14:1039-1044.

Silva GM, Vogel C (2016) Quantifying gene expression: the importance of being subtle. Mol Syst Biol 12:885.

Siucinska E, Kossut M (1996). Short-lasting classical conditioning induces reversible changes of representational maps of vibrissae in mouse SI cortex--a 2DG study. Cereb Cortex 6(3), 506-513.

Smith GB, Sederberg A, Elyada YM, Van Hooser SD, Kaschube M, Fitzpatrick D (2015) The development of cortical circuits for motion discrimination. Nat Neurosci 18:252-261.

Smith RS, Araneda RC (2010) Cholinergic modulation of neuronal excitability in the accessory olfactory bulb. J Neurophysiol 104:2963-2974.

Smith RS, Hu R, DeSouza A, Eberly CL, Krahe K, Chan W, Araneda RC (2015) Differential Muscarinic Modulation in the Olfactory Bulb. J Neurosci 35:1077310785 .

Sosulski DL, Bloom ML, Cutforth T, Axel R, Datta SR (2011) Distinct representations of olfactory information in different cortical centres Nature 472:213-216 doi:10.1038/nature09868

Spencer DG, Jr., Horvath E, Traber J (1986) Direct autoradiographic determination of M1 and M2 muscarinic acetylcholine receptor distribution in the rat brain: relation to cholinergic nuclei and projections. Brain Res 380:59-68.

Spors H, Grinvald A (2002) Spatio-temporal dynamics of odor representations in the mammalian olfactory bulb Neuron 34:301-315

Stettler DD, Axel R (2009) Representations of odor in the piriform cortex Neuron 63:854-864 doi:10.1016/j.neuron.2009.09.005

Storace DA, Cohen LB (2017) Measuring the olfactory bulb input-output transformation reveals a contribution to the perception of odorant concentration invariance Nat Commun 8:81 doi:10.1038/s41467-017-00036-2

Sullivan RM, Wilson DA (1991) Neural correlates of conditioned odor avoidance in infant rats. Behav Neurosci 105:307-312. 
Sullivan RM, Wilson DA (1995) Dissociation of behavioral and neural correlates of early associative learning. Dev Psychobiol 28:213-219.

Sullivan RM, Landers M, Yeaman B, Wilson DA (2000) Good memories of bad events in infancy. Nature 407:38-39.

Sullivan RM, Stackenwalt G, Nasr F, Lemon C, Wilson DA (2000) Association of an odor with activation of olfactory bulb noradrenergic beta-receptors or locus coeruleus stimulation is sufficient to produce learned approach responses to that odor in neonatal rats Behav Neurosci 114:957-962

Sullivan RM, Wilson DA (2003) Molecular biology of early olfactory memory Learn Mem 10:1-4 doi:10.1101/lm.58203

Sullivan RM, Wilson DA, Lemon C, Gerhardt GA (1994) Bilateral 6-OHDA lesions of the locus coeruleus impair associative olfactory learning in newborn rats Brain Res 643:306-309

Sullivan RM, Wilson DA, Leon M (1989) Norepinephrine and learning-induced plasticity in infant rat olfactory system J Neurosci 9:3998-4006

Sullivan SL, Ressler KJ, Buck LB (1995) Spatial patterning and information coding in the olfactory system Curr Opin Genet Dev 5:516-523

Sweatt JD (2016) Neural plasticity and behavior - sixty years of conceptual advances J Neurochem 139 Suppl 2:179-199 doi:10.1111/jnc.13580

Tavakoli A, Schmaltz A, Schwarz D, Margrie TW, Schaefer AT, Kollo M (2018). Quantitative Association of Anatomical and Functional Classes of Olfactory Bulb Neurons. J Neurosci 38(33), 7204-7220. doi: 10.1523/JNEUROSCI.030318.2018.

Tillerson JL, Caudle WM, Parent JM, Gong C, Schallert T, Miller GW (2006) Olfactory discrimination deficits in mice lacking the dopamine transporter or the $\mathrm{D} 2$ dopamine receptor. Behav Brain Res 172:97-105.

Tong J, Okutani F, Murata Y, Taniguchi M, Namba T, Wang YJ, Kaba H (2017) Tunicamycin impairs olfactory learning and synaptic plasticity in the olfactory bulb Neuroscience 344:371-379 doi:10.1016/j.neuroscience.2017.01.001

Tong MT, Peace ST, Cleland TA (2014) Properties and mechanisms of olfactory learning and memory Front Behav Neurosci 8:238 doi:10.3389/fnbeh.2014.00238

Treloar HB, Miller AM, Ray A, Greer CA (2010) Development of the Olfactory System. In: Menini A (ed) The Neurobiology of Olfaction. Frontiers in Neuroscience. Boca Raton (FL),

Trombley PQ, Westbrook GL (1990) Excitatory synaptic transmission in cultures of rat olfactory bulb. J Neurophysiol 64:598-606.

Tsuno Y, Kashiwadani H, Mori K (2008) Behavioral state regulation of dendrodendritic synaptic inhibition in the olfactory bulb. J Neurosci 28:9227-9238.

Vaaga CE, Westbrook GL (2016). Parallel processing of afferent olfactory sensory information. J Physiol 594(22), 6715-6732. doi: 10.1113/JP272755.

Valtcheva S, Froemke RC (2019). Neuromodulation of maternal circuits by oxytocin. Cell Tissue Res 375(1), 57-68. doi: 10.1007/s00441-018-2883-1.

Verhagen JV, Wesson DW, Netoff TI, White JA, Wachowiak M (2007) Sniffing controls an adaptive filter of sensory input to the olfactory bulb. Nat Neurosci 10:631-639.

Wachowiak M, Cohen LB (2001) Representation of odorants by receptor neuron input to the mouse olfactory bulb Neuron 32:723-735 
Wachowiak M, Economo MN, Diaz-Quesada M, Brunert D, Wesson DW, White JA, Rothermel M (2013) Optical dissection of odor information processing in vivo using GCaMPs expressed in specified cell types of the olfactory bulb J Neurosci 33:5285-5300 doi:10.1523/JNEUROSCI.4824-12.2013

Walker DL, Paschall GY, Davis M (2005) Glutamate receptor antagonist infusions into the basolateral and medial amygdala reveal differential contributions to olfactory vs. context fear conditioning and expression. Learn Mem 12:120-129.

Wang YJ, Okutani F, Murata Y, Taniguchi M, Namba T, Kaba H (2013) Histone acetylation in the olfactory bulb of young rats facilitates aversive olfactory learning and synaptic plasticity Neuroscience 232:21-31 doi:10.1016/j.neuroscience.2012.12.015

Wang Z, Edwards JG, Riley N, Provance DW, Jr., Karcher R, LI XD, Davidson IG, Ikebe M, Mercer JA, Kauer JA, Ehlers MD (2008) Myosin Vb mobilizes recycling endosomes and AMPA receptors for postsynaptic plasticity Cell 135:535-548 doi:10.1016/j.cell.2008.09.057

Weinberger, NM (1995). Dynamic regulation of receptive fields and maps in the adult sensory cortex. Annu Rev Neurosci 18, 129-158. doi: 10.1146/annurev.ne.18.030195.001021.

Weinberger NM (1998) Physiological memory in primary auditory cortex: characteristics and mechanisms Neurobiol Learn Mem 70:226-251 doi:10.1006/nlme.1998.3850

Weinberger NM (2004). Specific long-term memory traces in primary auditory cortex. Nat Rev Neurosci 5(4), 279-290. doi: 10.1038/nrn1366.

Weinberger NM (2007) Auditory associative memory and representational plasticity in the primary auditory cortex Hear Res 229:54-68 doi:10.1016/j.heares.2007.01.004

Weldon DA, Travis ML, Kennedy DA (1991) Posttraining D1 receptor blockade impairs odor conditioning in neonatal rats. Behav Neurosci 105:450-458.

Wellis DP, Kauer JS (1993) GABAA and glutamate receptor involvement in dendrodendritic synaptic interactions from salamander olfactory bulb. J Physiol 469:315-339.

Wesson DW, Carey RM, Verhagen JV, Wachowiak M (2008) Rapid encoding and perception of novel odors in the rat. PLoS Biol 6:e82.

Wilensky AE, Schafe GE, LeDoux JE (1999) Functional inactivation of the amygdala before but not after auditory fear conditioning prevents memory formation. $\mathrm{J}$ Neurosci 19:RC48.

Williamson SM, Moffat C, Gomersall MA, Saranzewa N, Connolly CN, Wright GA (2013) Exposure to acetylcholinesterase inhibitors alters the physiology and motor function of honeybees. Front Physiol 4:13.

Wilson DA (2001) Scopolamine enhances generalization between odor representations in rat olfactory cortex Learn Mem 8:279-285 doi:10.1101/1m.42601

Wilson DA, Fletcher ML, Sullivan RM (2004) Acetylcholine and olfactory perceptual learning. Learn Mem 11:28-34.

Wilson DA, Leon M (1988) Noradrenergic modulation of olfactory bulb excitability in the postnatal rat Brain Res 470:69-75

Wilson DA, Pham TC, Sullivan RM (1994) Norepinephrine and posttraining memory consolidation in neonatal rats Behav Neurosci 108:1053-1058 
Wilson MA, Fadel JR (2017) Cholinergic regulation of fear learning and extinction. J Neurosci Res 95:836-852.

Woo CC, Oshita MH, Leon M (1996) A learned odor decreases the number of Fosimmunopositive granule cells in the olfactory bulb of young rats Brain Res 716:149-156 doi:10.1016/0006-8993(96)00037-6

Yamada Y, Bhaukaurally K, Madarasz TJ, Pouget A, Rodriguez I, Carleton A (2017). Context- and Output Layer-Dependent Long-Term Ensemble Plasticity in a Sensory Circuit. Neuron 93(5), 1198-1212 e1195. doi: 10.1016/j.neuron.2017.02.006.

Ye L, Haroon MA, Salinas A, Paukert M (2017) Comparison of GCaMP3 and GCaMP6f for studying astrocyte $\mathrm{Ca} 2+$ dynamics in the awake mouse brain PLoS One 12:e0181113 doi:10.1371/journal.pone.0181113

Yu D, Ponomarev A, Davis RL (2004) Altered representation of the spatial code for odors after olfactory classical conditioning; memory trace formation by synaptic recruitment Neuron 42:437-449

Yu Y, Burton SD, Tripathy SJ, Urban NN (2015) Postnatal development attunes olfactory bulb mitral cells to high-frequency signaling J Neurophysiol 114:28302842 doi:10.1152/jn.00315.2015

Yuan Q, Harley CW, McLean JH (2003) Mitral cell betal and 5-HT2A receptor colocalization and cAMP coregulation: a new model of norepinephrine-induced learning in the olfactory bulb Learn Mem 10:5-15 doi:10.1101/1m.54803

Yuan Q, Harley CW (2014). Learning modulation of odor representations: new findings from Arc-indexed networks. Front Cell Neurosci 8, 423. doi: 10.3389/fncel.2014.00423.

Yuan Q, Shakhawat AM, Harley CW (2014) Mechanisms underlying early odor preference learning in rats. Prog Brain Res 208:115-156.

Yuan Q, Harley CW, McLean JH, Knopfel T (2002) Optical imaging of odor preference memory in the rat olfactory bulb. J Neurophysiol 87:3156-3159.

Zaborszky L, Carlsen J, Brashear HR, Heimer L (1986) Cholinergic and GABAergic afferents to the olfactory bulb in the rat with special emphasis on the projection neurons in the nucleus of the horizontal limb of the diagonal band. J Comp Neurol 243:488-509.

Zak JD, Whitesell JD, Schoppa NE (2015) Metabotropic glutamate receptors promote disinhibition of olfactory bulb glomeruli that scales with input strength. J Neurophysiol 113:1907-1920.

Zaman J, Ceulemans E, Hermans D, Beckers T (2019). Direct and indirect effects of perception on generalization gradients. Behav Res Ther 114, 44-50. doi: 10.1016/j.brat.2019.01.006.

Zarzecki P, Witte S, Smits E, Gordon DC, Kirchberger P, Rasmusson DD (1993). Synaptic mechanisms of cortical representational plasticity: somatosensory and corticocortical EPSPs in reorganized raccoon SI cortex. J Neurophysiol 69(5), 1422-1432. doi: 10.1152/jn.1993.69.5.1422.

Zhang G, Stackman RW, Jr. (2015) The role of serotonin 5-HT2A receptors in memory and cognition. Front Pharmacol 6:225.

Zhang JJ, Okutani F, Huang GZ, Taniguchi M, Murata Y, Kaba H (2010) Common properties between synaptic plasticity in the main olfactory bulb and olfactory 
learning in young rats Neuroscience 170:259-267 doi:

10.1016/j.neuroscience.2010.06.002

Zhang JJ, Okutani F, Inoue S, Kaba H (2003) Activation of the cyclic AMP response element-binding protein signaling pathway in the olfactory bulb is required for the acquisition of olfactory aversive learning in young rats Neuroscience 117:707-713

Zhang X, Li B (2018). Population coding of valence in the basolateral amygdala. Nat Commun 9(1), 5195. doi: 10.1038/s41467-018-07679-9. 


\section{VITA}

Jordan Marie Ross was born in Atlanta, GA in 1991, and was raised in the surrounding metro area. Jordan matriculated into North Georgia College and State University in August 2009 and received a Bachelor of Science in Psychological Science from the recently consolidated University of North Georgia. During her undergraduate years she married her interest in psychology with rigorous biological and statistical methods, working in two undergraduate research laboratories. It was through undergraduate research that she discovered and explored her interest in Neuroscience. Jordan entered the University of Tennessee Health Science Center Ph.D. program in Neuroscience in fall of 2013. In March of 2015, Jordan joined the lab of Dr. Max Fletcher where she used the olfactory system in combination with awake calcium imaging and behavioral assays to study basic mechanisms of fear learning and generalization. During her time at UTHSC, Jordan completed many personal and scientific goals such as finishing a half marathon, watching every Marvel Cinematic Universe Movie (as "required" by her mentor), receiving an NIH predoctoral fellowship, and publishing several first author peer reviewed papers. Jordan will receive her Ph.D. in August 2019. While she is sad to leave the Fletcher Lab family, Jordan is excited to continue her scientific training as a postdoc at Georgia State University in the lab of Jordan P. Hamm. 